\title{
Rh-Catalyzed Stereospecific Synthesis of Allenes from Propargylic Benzoates and Arylboronic Acids
}

\author{
Jonathan Ruchti and Erick M. Carreira* \\ Laboratorium für Organische Chemie, ETH Zürich, 8093 Zürich, Switzerland \\ *E-mail: carreira@org.chem.ethz.ch
}

\section{Supporting Information}

\section{Table of Contents}

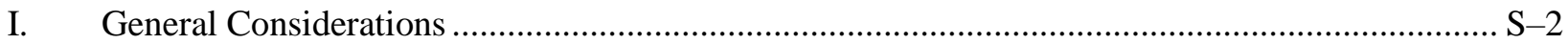

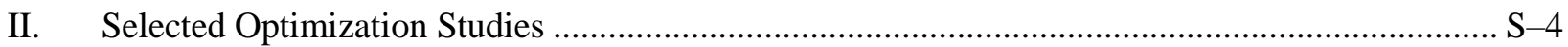

III. Synthesis and Characterization of Starting Materials and Ligand 4 ....................................... S-6

IV. Synthesis and Characterization of Products ........................................................................... S-16

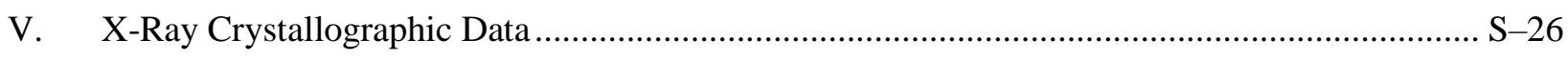

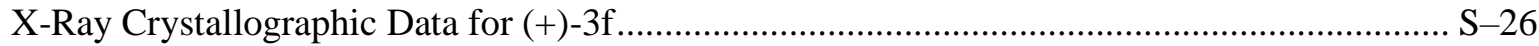

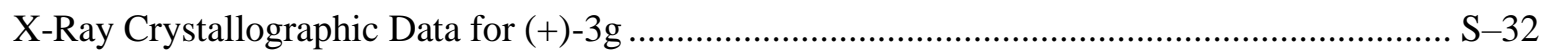

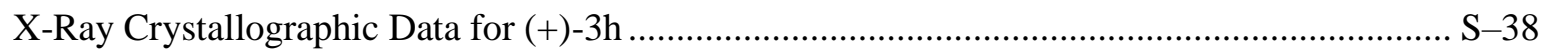

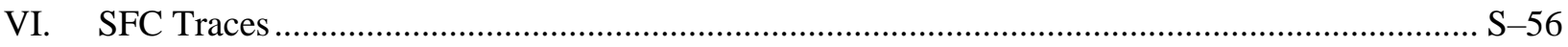

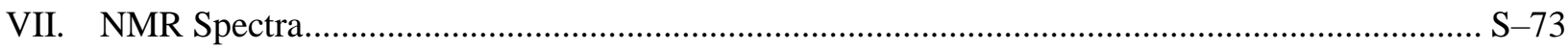




\section{General Considerations}

Names and stereochemistry. Names of compounds were generated using the ChemBioDraw 12.0 (Cambridgesoft) software. For racemic compounds no stereochemistry is indicated and for the X-ray diffraction derived plot of $\mathbf{3 g}$ and $\mathbf{3 h}$ an arbitrary enantiomer is shown. The absolute stereochemistry for compounds 3a-k is assigned based on X-ray data obtained for $\mathbf{3 f}$. The absolute stereochemistry of the chiral propargylic alcohols and benzoates is assigned in correlation to reported data.

Solvents and reagents. 2,2'-Biphenol (Fluka), $\mathrm{PCl}_{3}$ (Aldrich), $5 H$-dibenzo[ $b$ f $]$ azepine (CombiBlocks), $\mathrm{Zn}(\mathrm{OTf})_{2}$ (TCI, opened and stored in a glovebox), (-)- $N$-methylephedrine (Fluka), $n$-BuLi in hexanes (1.60 M, Aldrich), 4-bromo-1-butyne (Aldrich), (s)-5,5-diphenyl-2-methyl-3,4-propano1,3,2-oxazaborolidine ((S)-2-Methyl-CBS-oxazaborolidine, CAS 112022-81-8, TCI), $\mathrm{BH}_{3} \bullet \mathrm{SMe}_{2}$ (Aldrich), N,N-dimethylpyridin-4-amine (DMAP, Fluka), benzoyl chloride (Aldrich), 1,2-dichloroethane (Fluka, stored under ambient air), $\mathrm{K}_{3} \mathrm{PO}_{4}$ (Acros), [ $\left.\{\mathrm{Rh}(\operatorname{cod}) \mathrm{Cl}\}_{2}\right]$ (98\%, CombiBlocks), [\{ $\left.\mathrm{Rh}(\operatorname{cod}) \mathrm{OH}\}_{2}\right]$ (95\%, Aldrich), phenylboronic acid (TCI), 2-methylphenylboronic acid (Combi-Blocks), 4-methoxyphenylboronic acid (Aldrich), 2,3-dihydrobenzo[b][1,4]dioxin-6ylboronic acid (Combi-Blocks), 1-naphthaleneboronic acid (Aldrich), 4-bromophenylboronic acid (Aldrich), 4-biphenylboronic acid (Apollo Scientific), 3,4-dimethoxybenzeneboronic acid (Apollo Scientific), 4-iodophenylboronic acid (Aldrich), 3-bromophenylboronic acid (Combi-Blocks) were used as received. All other chemicals and solvents were purchased from ABCR, Acros, Apollo, Brunschwig, Combi-Blocks, EGT Chemie, Fisher, Fluka, Fluorochem, Hänseler, Lancaster, Merck, Scharlau, Sigma-Aldrich, TCI, Thommen-Furler, Univar and used as such unless otherwise stated and with the exception of dried solvents. $\mathrm{CH}_{2} \mathrm{Cl}_{2}, 1$,4-dioxane, DMF, THF and toluene were dried by passage over two $4 \times 36$ inch columns of anhydrous neutral A-2 alumina (Macherey und Nagel; activated for $>12 \mathrm{~h}$ at $300{ }^{\circ} \mathrm{C}$ under a flow of $\mathrm{N}_{2}$ ) under an atmosphere of $\mathrm{N}_{2} . \mathrm{MeOH}$ was distilled from magnesium turnings under an atmosphere of dry $\mathrm{N}_{2}$. $\mathrm{Et}_{3} \mathrm{~N}$ was distilled from $\mathrm{CaH}_{2}$ under an atmosphere of dry $\mathrm{N}_{2}$. Pyridine was distilled from $\mathrm{KOH}$ under an atmosphere of dry $\mathrm{N}_{2}$. Deuterated solvents were obtained from Armar Chemicals, Döttingen, Switzerland.

Reaction handling. All non-aqueous reactions were performed in flame-dried glassware under an atmosphere of $\mathrm{N}_{2}$ unless stated otherwise. Reactions were magnetically stirred and monitored by analytical thin layer chromatography (TLC) unless otherwise noted. TLC was performed on Merck silica gel $60 \mathrm{~F}_{254}$ TLC glass plates and visualized with UV fluorescence quenching and cerium 
ammonium molybdate (CAM) or $\mathrm{KMnO}_{4}$ stain. Column chromatographic purification was performed as flash chromatography on silica gel with $0.2-0.5$ bar pressure using silica gel from Fluka (pore size $60 \AA$, 230 - 400 mesh particle size) or SiliCycle (SilaFlash ${ }^{\circledR}$ P60, 230 - 400 mesh particle size) and technical grade solvents. Concentrations under reduced pressure were performed by rotary evaporation at $42{ }^{\circ} \mathrm{C}$ at the appropriate pressure. The yields given refer to the purified products, unless otherwise stated.

Melting points. Melting points were measured on a Büchi SMP-20 melting point apparatus using open glass capillaries and are uncorrected. The solvent from which the compound was recrystallized is given in parentheses. If no solvent is given, the melting point refers to the solid product as obtained after the workup or purification described in the experimental procedure.

NMR spectroscopy. NMR data was recorded on a Bruker AVIII400 and Bruker DRX400 spectrometer, both operating at $400 \mathrm{MHz}$ for ${ }^{1} \mathrm{H}$ acquisitions. Measurements were carried out at $298 \mathrm{~K}$. Chemical shifts $(\delta)$ are reported in parts per million $(\mathrm{ppm})$ with the solvent resonance as internal standard for ${ }^{1} \mathrm{H}$ spectroscopy (chloroform $\mathrm{CHCl}_{3}$ singlet at $7.26 \mathrm{ppm}$ ) and for ${ }^{13} \mathrm{C}$ spectroscopy $\left(\mathrm{CDCl}_{3}\right.$ triplet at $77.16 \mathrm{ppm}) .{ }^{1} \mathrm{~J}$ is the coupling constant in hertz $(\mathrm{Hz})$; the multiplicities are abbreviated s, singlet; d, doublet; t, triplet; q, quartet; quin, quintet; m, multiplet or unresolved; br, broad signal; app, apparent. All ${ }^{13} \mathrm{C}$ spectra were measured with complete proton decoupling.

IR spectroscopy. Infrared spectra were recorded on a Perkin Elmer UATR Spectrum Two FT-IR spectrometer. Absorptions are given in reciprocal centimeters, and the absorptions are s, strong; m, medium; w, weak; br, broad signal.

Mass spectrometry. Mass spectrometric analyses were performed by the mass spectrometry service of the Laboratorium für Organische Chemie at ETH Zürich by L. Bertschi, O. Greter and R. Häfliger under direction of Dr. X. Zhang. ESI measurements were carried out on Bruker maXis - ESI-Qq-TOF-MS and Bruker solariX - ESI-FTICR-MS. EI measurements were carried out on a Micromass (Waters) AutoSpec Ultima - EI-Sector-MS. The following abbreviations are used: HRMS is high-resolution mass spectrometry (massspectrometric accurate mass), $m / z$ is the mass-tocharge ratio, $\mathrm{M}$ is the molecular weight of the molecule itself, $[\mathrm{M}]^{+}$is the molecular ion.

Specific Rotation. Specific rotations $(\alpha)$ were measured on a Jasco P-2000 polarimeter at the sodium D line with a $100 \mathrm{~mm}$ path length cell. Values are reported as follows: $[\alpha]_{\mathrm{D}}^{\mathrm{T}}$ in parentheses 
concentration $\left(\mathrm{c}=1.00\right.$ corresponds to $\left.10.0 \mathrm{mg} \cdot \mathrm{mL}^{-1}\right)$, and solvent. The temperature $(\mathrm{T})$ at which the determination was made is given as the superscript number $\left(/{ }^{\circ} \mathrm{C}\right)$.

SFC. Supercritical fluid chromatography (SFC) was performed on a Jasco 2080 Plus and Waters Acquity UPC ${ }^{2}$ system under the conditions given for each measurement. Racemic samples were prepared by conducting the same reactions as for the enantioenriched samples but in a racemic fashion. ${ }^{1}$

X-ray diffraction. X-ray diffraction experiments have been carried out by Dr. N. Trapp and M. Solar from the Small Molecule Crystallography Center (SMoCC) at the Department of Chemistry and Applied Biosciences at ETH Zürich.

\section{Selected Preliminary Results}

\section{Selected Ligand Screening (Table 1).}
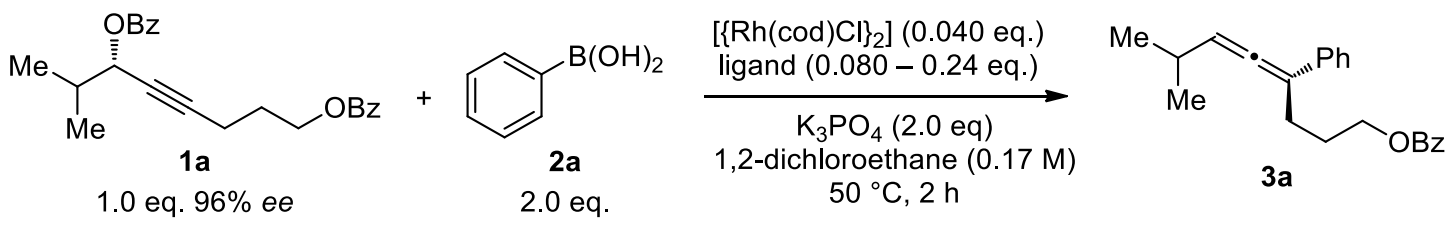

\begin{tabular}{cccccc}
\hline Entry & Ligand & Ligand/Rh & Conversion & $\boldsymbol{e e}(\mathbf{3 a})$ & $\boldsymbol{e s}$ \\
\hline 1 & $\mathbf{4}$ & $2 / 1$ & $>95 \%$ & $94 \%$ & $98 \%$ \\
2 & $\mathbf{5}$ & $2 / 1$ & $46 \%$ & $94 \%$ & $98 \%$ \\
3 & $\mathbf{6}$ & $2 / 1$ & $<5 \%$ & n. d. & n. d. \\
4 & $\mathbf{7}$ & $1 / 1$ & $>95 \%$ & $85 \%$ & $89 \%$ \\
5 & $\mathrm{DPPF}(\mathbf{8})$ & $1 / 1$ & $>95 \%$ & $87 \%$ & $91 \%$ \\
6 & $\mathbf{9}$ & $1 / 1$ & $>95 \%$ & $90 \%$ & $94 \%$ \\
7 & $\mathrm{P}(\mathrm{OMe})_{3}(\mathbf{1 0})$ & $3 / 1$ & $61 \%$ & $80 \%$ & $83 \%$ \\
\hline
\end{tabular}

\footnotetext{
${ }^{1}$ For example by using LDA, $n$ BuLi or ( \pm )- $N$-methylephedrine for the addition of alkynes to aldehydes and then following the same general procedures for the next two steps. A typical procedure for such a racemic addition is given in the following. 
<smiles>c1ccc2c(c1)OP(Oc1cccc3ccccc13)c1ccccc1-2</smiles>

4

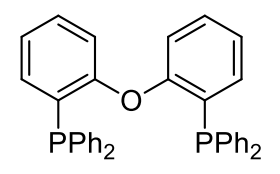

7<smiles>c1ccc(-c2ccccc2Op2oc3ccccc3n3cccccc2-3)cc1</smiles>

5

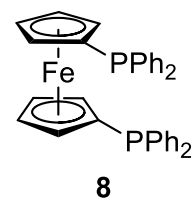

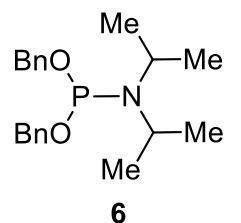

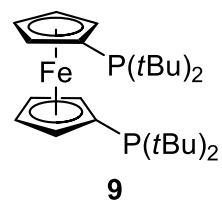

Typical procedure for the ligand screening: To a suspension of benzoate 1a $(18 \mathrm{mg}, 0.050 \mathrm{mmol}$, 1.0 equiv.), phenylboronic acid (12 mg, $0.10 \mathrm{mmol}, 2.0$ equiv.), $\mathrm{K}_{3} \mathrm{PO}_{4}$ (21 mg, $0.10 \mathrm{mmol}, 2.0$ equiv.) in 1,2-dichloroethane $(0.20 \mathrm{~mL})$ was added a solution of $\left[\{\mathrm{Rh}(\operatorname{cod}) \mathrm{Cl}\}_{2}\right](1.0 \mathrm{mg}, 2.0 \mu \mathrm{mol}$, 0.040 equiv.), the appropriate ligand (0.080 equiv., 0.16 equiv., or 0.24 equiv.) in 1,2-dichloroethane $(0.10 \mathrm{~mL})$. The resulting suspension was sparged with $\mathrm{N}_{2}$ and stirred in a screw-capped vial at $50{ }^{\circ} \mathrm{C}$ for $2 \mathrm{~h}$. It was then diluted with $\mathrm{Et}_{2} \mathrm{O}$ and filtered through a plug of silica gel with copious washings $\left(\mathrm{Et}_{2} \mathrm{O}\right)$. The solution was concentrated and the conversion was determined by ${ }^{1} \mathrm{H}$ NMR of the unpurified reaction mixture. The enantiomeric excess was determined by supercritical fluid chromatography (SFC) on a chiral stationary phase after purification by flash column chromatography on silica gel.

\section{Reaction with Other Phenyl Boron Nucleophiles (Table SI-1). ${ }^{[a]}$}
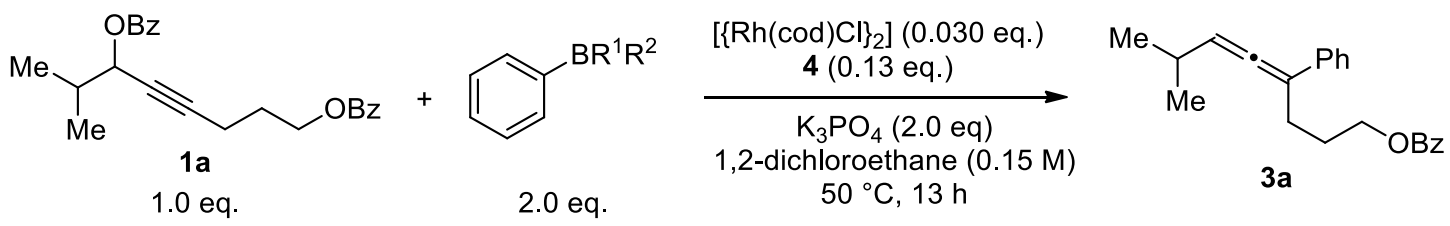

\begin{tabular}{ccc}
\hline Entry & PhBR $^{\mathbf{1}} \mathbf{R}^{\mathbf{2}}$ & Conversion \\
\hline 1 & $\mathrm{PhB}(\mathrm{OH})_{2}$ & $>95 \%$ \\
2 & $\mathrm{PhBF}_{3} \mathrm{~K}$ & $<5 \%$ \\
3 & $\mathrm{PhBpin}$ & $24 \%$ \\
4 & $\mathrm{PhBneop}$ & $35 \%$ \\
5 & $(\mathrm{PhBO})_{3}$ & $81 \%$ \\
\hline
\end{tabular}

[a] $\mathrm{PhBpin}=$ phenyl pinacol boronic ester, $\mathrm{PhBneop}=$ phenyl boronic acid neopentylglycol ester, $(\mathrm{PhBO})_{3}=$ phenylboroxine. 
Procedure for the reaction with various phenyl boron nucleophiles: To a suspension of benzoate 1a (9.1 mg, $0.025 \mathrm{mmol}, 1.0$ equiv.), the corresponding phenyl boron reagent (0.050 mmol, 2.0 equiv.), $\mathrm{K}_{3} \mathrm{PO}_{4}(11 \mathrm{mg}, 0.050 \mathrm{mmol}, 2.0$ equiv. $)$ in 1,2-dichloroethane $(0.12 \mathrm{~mL})$ was added $50 \mu \mathrm{L}$ of a stock solution of $\left[\left\{\mathrm{Rh}(\mathrm{cod}) \mathrm{Cl}_{2}\right](2.2 \mathrm{mg}, 4.5 \mu \mathrm{mol}-0.75 \mu \mathrm{mol}, 0.030\right.$ equiv. per reaction), 4 (7.6 mg, $0.019 \mathrm{mmol}-3.1 \mu \mathrm{mol}, 0.13$ equiv. per reaction) in 1,2-dichloroethane $(0.30 \mathrm{~mL})$. The resulting suspension was sparged with $\mathrm{N}_{2}$ and stirred in a screw-capped vial at $50{ }^{\circ} \mathrm{C}$ for $13 \mathrm{~h}$. It was then diluted with $\mathrm{Et}_{2} \mathrm{O}$ and filtered through a plug of silica gel with copious washings $\left(\mathrm{Et}_{2} \mathrm{O}\right)$. The solution was concentrated and the conversion was determined by ${ }^{1} \mathrm{H}$ NMR of the unpurified reaction mixture.

\section{Example for a Propargylic Carbonate.}
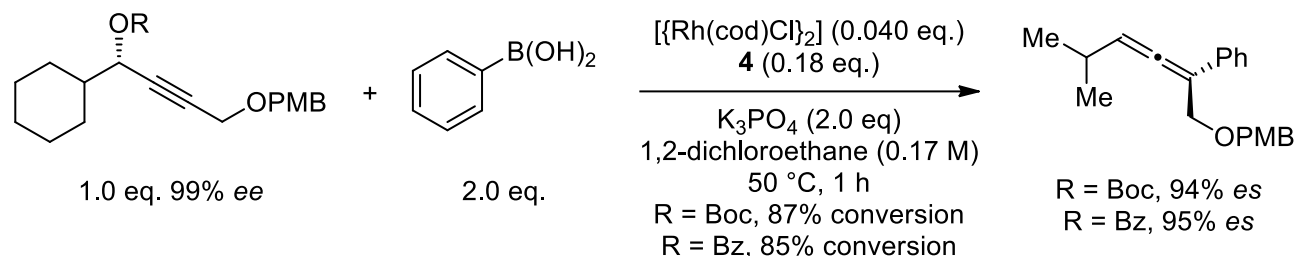

III. Synthesis and Characterization of Starting Materials and Ligand 4<smiles>C1=Cc2ccccc2N(POc2ccccc2-c2ccccc2)c2ccccc21</smiles>

5-(Dibenzo $[d, f][1,3,2]$ dioxaphosphepin-6-yl)-5H-dibenzo $[b, f]$ azepine (4). Following a modified literature procedure, ${ }^{2}$ a $50 \mathrm{~mL}$ Schlenk flask was charged with 2,2'-biphenol (1.86 g, $10.0 \mathrm{mmol}$, 1.00 equiv.). $\mathrm{PCl}_{3}$ (8.60 mL, $98.6 \mathrm{mmol}, 9.86$ equiv.) and $\mathrm{DMF}$ (50 $\mu \mathrm{L}, 0.65 \mathrm{mmol}, 0.065$ equiv.) were added at $23{ }^{\circ} \mathrm{C}$ and the resulting brown suspension was was heated to $50{ }^{\circ} \mathrm{C}$ for $1 \mathrm{~h}$. The now brown solution was left stirring at $50{ }^{\circ} \mathrm{C}$ while excess $\mathrm{PCl}_{3}$ was removed through short path distillation into

\footnotetext{
${ }^{2}$ Defieber, C.; Ariger, M. A.; Moriel, P.; Carreira, E. M. Angew. Chem., Int. Ed. 2007, 46, 3139. 
a flask cooled to $-78{ }^{\circ} \mathrm{C}\left(\mathrm{PCl}_{3}\right.$ was later quenched with sat. aq. $\left.\mathrm{NaHCO}_{3}\right)$. To the remaining oil was added toluene $(2 \times 2.0 \mathrm{~mL})$ which was removed under reduced pressure. The Schlenk flask was evacuated for $10 \mathrm{~min}$ and $30 \mathrm{~min}$ after the first and second cycle respectively. The flask was allowed to cool to $23{ }^{\circ} \mathrm{C}$ and the oil was dissolved in THF (20 mL). In a separate $200 \mathrm{~mL}$, single-necked, roundbottomed flask a solution of $5 H$-dibenzo[b,f]azepine $(2.03 \mathrm{~g}, 10.5 \mathrm{mmol}, 1.05$ equiv.) in THF (20 mL) was treated with $n$-BuLi in hexanes $\left(1.60 \mathrm{M}, 6.25 \mathrm{~mL}, 10.0 \mathrm{mmol}, 1.00\right.$ equiv.) at $-78{ }^{\circ} \mathrm{C}$. The resulting dark violet mixture was stirred at $-78^{\circ} \mathrm{C}$ for $2 \mathrm{~h}$ before the solution of the phosphorchloridite in THF was added via cannula. The Schlenk flask was rinsed with THF (10 mL). The dark green mixture was allowed to warm slowly to $14^{\circ} \mathrm{C}$ over $18 \mathrm{~h}$. The now orange solution was concentrated to give an orange gum. The crude product was suspended in $30 \mathrm{~mL}$ cyclohexane/toluene $2: 1$ containing $0.5 \%$ $\mathrm{Et}_{3} \mathrm{~N}$. Celite (18 g) was added and the volatiles were removed under reduced pressure. The solid was loaded onto a column and the product was obtained after flash column chromatography on silica gel (cyclohexane/toluene 2:1 with $0.5 \% \mathrm{Et}_{3} \mathrm{~N}$ ) as a white solid $(3.15 \mathrm{~g}, 7.73 \mathrm{mmol}, 77 \%)$. The title compound was stored under an inert atmosphere in a freezer $\left(-20{ }^{\circ} \mathrm{C}\right)$.

TLC: $R_{f}=0.51$ (cyclohexane/toluene 2:1 with $0.5 \% \mathrm{Et}_{3} \mathrm{~N}$; UV, CAM); Melting point: $164-165^{\circ} \mathrm{C} ;{ }^{1} \mathbf{H}-\mathbf{N M R}\left(400 \mathrm{MHz}, \mathrm{CDCl}_{3}, 298 \mathrm{~K}\right): \delta 7.39-7.36(\mathrm{~m}, 2 \mathrm{H}), 7.29-7.09$ (m, 12H), 7.03 - $7.02(\mathrm{~m}, 2 \mathrm{H}), 6.97$ (s, 2H); ${ }^{13} \mathrm{C}-\mathbf{N M R}\left(101 \mathrm{MHz}, \mathrm{CDCl}_{3}, 298 \mathrm{~K}\right): \delta 151.0,150.9,142.8,142.7$, 136.14, 136.12, 131.5 (two coincident resonances), 130.62, 130.59, 129.64, 129.63, 129.2 (two coincident resonances), 129.05, 128.99, 128.96 (four coincident resonances), 126.7 (two coincident resonances), 124.44, 124.43, 122.12, 122.11; ${ }^{31} \mathbf{P}\left\{{ }^{\mathbf{1}} \mathbf{H}\right\}$-NMR $\left(162 \mathrm{MHz}, \mathrm{CDCl}_{3}, 298 \mathrm{~K}\right): \delta 137.8$; IR (neat): 3024 (w), 1599 (w), 1567 (w), 1497 (w), 1486 (m), 1475 (w), 1459 (w), 1435 (m), 1281 (w), 1243 (m), 1196 (s), 1185 (m), 1165 (w), 1153 (w), 1117 (w), 1105 (w), 1095 (m), 1040 (w), 1008 (w), $984(\mathrm{~m}), 946(\mathrm{w}), 920(\mathrm{w}), 891$ (s), $885(\mathrm{~s}), 861(\mathrm{~m}), 848(\mathrm{~m}), 836(\mathrm{~m}), 800(\mathrm{~m}), 774(\mathrm{~s}), 769$ (s), 760 (s), 747 (s), 731 (m), $711(\mathrm{~m}), 699(\mathrm{~m}), 678(\mathrm{~m}), 623$ (w), 596 (m), 553 (m), 542 (m), 519 (m), $511(\mathrm{~m}), 485$ (s), $474(\mathrm{~m}), 462(\mathrm{~m}) \mathrm{cm}^{-1}$; HRMS (ESI) $\mathrm{m} / z$ : exact mass calculated for $\mathrm{C}_{26} \mathrm{H}_{19} \mathrm{NO}_{2} \mathrm{P}$ $[\mathrm{M}+\mathrm{H}]^{+}$408.1148, found 408.1148.

\section{General Procedure A: Enantioselective Addition of Terminal Alkynes to Aldehydes.} Following a slightly modified reported procedure, ${ }^{3}$ a $100 \mathrm{~mL}$, single-necked, round-bottomed flask

\footnotetext{
${ }^{3}$ Frantz, D. E.; Fassler, R.; Carreira, E. M. J. Am. Chem. Soc. 2000, 122, 1806.
} 
containing a magnetic stir bar, was charged in a glovebox with $\mathrm{Zn}(\mathrm{OTf})_{2}(2.40 \mathrm{~g}, 6.60 \mathrm{mmol}$, 1.10 equiv.). The flask was removed from the glovebox and heated to $140{ }^{\circ} \mathrm{C}$ for $2 \mathrm{~h}$ under vacuum (the initial pressure of $0.6 \mathrm{mbar}$ decreased to $0.1 \mathrm{mbar}$ within the first $20 \mathrm{~min}$ ). The flask was allowed to cool to $24{ }^{\circ} \mathrm{C}$ before (-)- $N$-methylephedrine $(1.29 \mathrm{~g}, 7.20 \mathrm{mmol}, 1.20$ equiv.) was added in a glovebox. The flask was sealed with a septum and removed from the glovebox. Toluene $(18.0 \mathrm{~mL}$, $0.333 \mathrm{M})$ and $\mathrm{Et}_{3} \mathrm{~N}\left(1.00 \mathrm{~mL}, 7.20 \mathrm{mmoL}, 1.20\right.$ equiv.) were added under $\mathrm{N}_{2}$ at $24{ }^{\circ} \mathrm{C}$. The white suspension was stirred at $24{ }^{\circ} \mathrm{C}$ for $2 \mathrm{~h}$, then the corresponding alkyne (6.00 mmol, 1.00 equiv.) was added in one portion. After $15 \mathrm{~min}$ the appropriate aldehyde (7.20 mmol, 1.20 equiv.) was added, again in one portion. The resulting suspension was stirred at $24{ }^{\circ} \mathrm{C}$ for the time indicated for each substrate. The reaction was quenched at $24{ }^{\circ} \mathrm{C}$ by addition of sat. aq. $\mathrm{NH}_{4} \mathrm{Cl}(60 \mathrm{~mL})$. The mixture was diluted with $\mathrm{Et}_{2} \mathrm{O}(180 \mathrm{~mL})$, the layers were separated and the aqueous layer was extracted with $\mathrm{Et}_{2} \mathrm{O}(50 \mathrm{~mL})$. The combined organic layers were washed with sat. aq. $\mathrm{NaCl}(120 \mathrm{~mL})$, dried over $\mathrm{MgSO}_{4}$, and filtered. The solution was concentrated, and the residue was purified by flash column chromatography on silica gel.

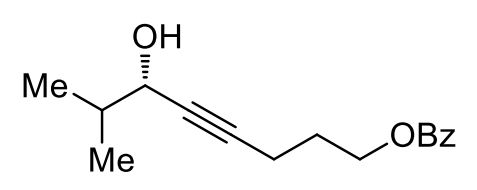

(+)-(S)-6-Hydroxy-7-methyloct-4-yn-1-yl benzoate (SI-1). General procedure A was followed on a $10.0 \mathrm{mmol}$ scale. The reaction was stirred at $24{ }^{\circ} \mathrm{C}$ for $15 \mathrm{~h}$. The crude product was purified by flash column chromatography on silica gel (cyclohexane/EtOAc 8:1 to 2:1 gradient). The title compound was isolated as a clear, colorless oil (2.29 g, $8.79 \mathrm{mmol}, 88 \%)$.

TLC: $R_{f}=0.32$ (cyclohexane/EtOAc 3:1; UV, CAM); Specific Rotation: $[\alpha]_{\mathrm{D}}^{22}+1.0(\mathrm{c}=2.00$, $\left.\mathrm{CHCl}_{3}\right) ;{ }^{1} \mathbf{H}$-NMR $\left(400 \mathrm{MHz}, \mathrm{CDCl}_{3}, 298 \mathrm{~K}\right): \delta 8.06-8.03(\mathrm{~m}, 2 \mathrm{H}), 7.56(\mathrm{tt}, J=6.9,1.3 \mathrm{~Hz}, 1 \mathrm{H})$, $7.46-7.42(\mathrm{~m}, 2 \mathrm{H}), 4.42(\mathrm{t}, J=6.3 \mathrm{~Hz}, 2 \mathrm{H}), 4.16-4.12(\mathrm{~m}, 1 \mathrm{H}), 2.42(\mathrm{td}, J=7.0,2.0 \mathrm{~Hz}, 2 \mathrm{H}), 1.99$ $(\operatorname{app~p}, J=6.7 \mathrm{~Hz}, 2 \mathrm{H}), 1.87-1.79(\mathrm{~m}, 2 \mathrm{H}), 0.98(\operatorname{app~t}, J=7.0 \mathrm{~Hz}, 6 \mathrm{H}) ;{ }^{13} \mathbf{C}-\mathbf{N M R}(101 \mathrm{MHz}$, $\left.\mathrm{CDCl}_{3}, 298 \mathrm{~K}\right): \delta 166.7,133.1,130.4,129.7,128.5,84.6,81.0,68.2,63.7,34.8,28.1,18.2,17.6,15.8$; IR (neat): 3424 (br), 3064 (w), 2960 (w), 2873 (w), 1717 (s), 1602 (w), 1584 (w), 1468 (w), 1452 (w), 1386 (w), 1366 (w), 1353 (w), 1315 (w), 1271 (s), 1177 (w), 1147 (w), 1115 (m), 1070 (w), 1026 (m), $980(\mathrm{w}), 935$ (w), 912 (w), $844(\mathrm{w}), 806$ (w), $749(\mathrm{w}), 710(\mathrm{~s}), 687(\mathrm{w}), 675$ (w) cm ${ }^{-1}$; HRMS (EI) 
m/z: exact mass calculated for $\mathrm{C}_{13} \mathrm{H}_{13} \mathrm{O}_{3}\left[\mathrm{M}-\mathrm{C}_{3} \mathrm{H}_{7}\right]^{+}$217.0860, found 217.0856; SFC (Jasco 2080 Plus, Daicel Chiralpak AS-H, $98 \% \mathrm{CO}_{2}, 2 \% \mathrm{MeOH}$ at 100 bar, flow rate $2.0 \mathrm{~mL} \cdot \mathrm{min}^{-1}, 25^{\circ} \mathrm{C}$, detection $219 \mathrm{~nm}$ ): $96 \%$ ee, $\mathrm{t}_{\mathrm{R}}$ (minor enantiomer) $9.1 \mathrm{~min}, \mathrm{t}_{\mathrm{R}}$ (major enantiomer) $9.4 \mathrm{~min}$.

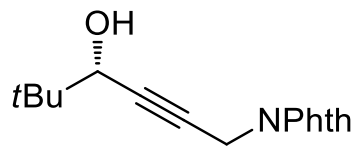

\section{(-)-(S)-2-(4-Hydroxy-5,5-dimethylhex-2-yn-1-yl)isoindoline-1,3-dione $\quad$ (SI-2). General} procedure A was followed on a $6.00 \mathrm{mmol}$ scale. The reaction was stirred at $24{ }^{\circ} \mathrm{C}$ for $12 \mathrm{~h}$. The crude product was purified by flash column chromatography on silica gel (cyclohexane/EtOAc 4:1 to 2:1 gradient). The title compound was isolated as a white solid $(1.37 \mathrm{~g}, 5.05 \mathrm{mmol}, 84 \%)$.

TLC: $R_{f}=0.36$ (cyclohexane/EtOAc 2:1; UV, CAM); Melting point: $94-95^{\circ} \mathrm{C}$; Specific Rotation: $[\alpha]_{\mathrm{D}}^{22}-0.7\left(\mathrm{c}=1.00, \mathrm{CHCl}_{3}\right) ;{ }^{1} \mathrm{H}-\mathrm{NMR}\left(400 \mathrm{MHz}, \mathrm{CDCl}_{3}, 298 \mathrm{~K}\right): \delta 7.89-7.84(\mathrm{~m}, 2 \mathrm{H})$, $7.75-7.70(\mathrm{~m}, 2 \mathrm{H}), 4.48(\mathrm{~d}, J=1.8 \mathrm{~Hz}, 2 \mathrm{H}), 3.98(\mathrm{dt}, J=6.1,1.8 \mathrm{~Hz}, 1 \mathrm{H}), 1.91(\mathrm{~d}, J=6.1 \mathrm{~Hz}, 1 \mathrm{H})$, 0.95 (s, 9H); ${ }^{13}$ C-NMR (101 MHz, $\left.\mathrm{CDCl}_{3}, 298 \mathrm{~K}\right): \delta$ 167.2, 134.3, 132.1, 123.7, 83.1, 79.2, 71.4, 36.0, 27.4, 25.4; IR (neat): 3446 (m), 2961 (w), $2869(\mathrm{w}), 1770(\mathrm{~m}), 1708(\mathrm{~s}), 1611(\mathrm{w}), 1478(\mathrm{w})$, $1465(\mathrm{w}), 1429(\mathrm{~m}), 1401(\mathrm{~m}), 1352(\mathrm{~m}), 1328(\mathrm{~m}), 1319(\mathrm{~m}), 1242(\mathrm{w}), 1181(\mathrm{w}), 1134(\mathrm{~m})$, $1122(\mathrm{~m}), 1088(\mathrm{w}), 1050(\mathrm{~m}), 1011(\mathrm{~m}), 946(\mathrm{~m}), 900(\mathrm{w}), 850(\mathrm{w}), 798(\mathrm{w}), 761(\mathrm{w}), 710(\mathrm{~s})$, 724 (s), 639 (m), 601 (w), $544(\mathrm{w}), 529$ (m), $463(\mathrm{w}) \mathrm{cm}^{-1}$; HRMS (EI) m/z: exact mass calculated for $\mathrm{C}_{12} \mathrm{H}_{9} \mathrm{NO}_{3}\left[\mathrm{M}-\mathrm{C}_{4} \mathrm{H}_{8}\right]^{+}$215.0577, found 215.0579; SFC (Jasco 2080 Plus, Daicel Chiralcel OJ-H, $98 \% \mathrm{CO}_{2}, 2 \% \mathrm{MeOH}$ at $100 \mathrm{bar}$, flow rate $2.0 \mathrm{~mL} \cdot \mathrm{min}^{-1}, 25^{\circ} \mathrm{C}$, detection $218 \mathrm{~nm}$ ): $96 \%$ ee, $\mathrm{t}_{\mathrm{R}}$ (minor enantiomer) $13.7 \mathrm{~min}, \mathrm{t}_{\mathrm{R}}$ (major enantiomer) $15.0 \mathrm{~min}$.

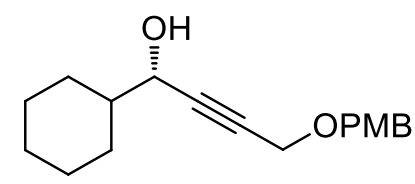

(+)-(S)-1-Cyclohexyl-4-((4-methoxybenzyl)oxy)but-2-yn-1-ol (SI-3). General procedure A was followed on a $4.00 \mathrm{mmol}$ scale. The reaction was stirred at $24{ }^{\circ} \mathrm{C}$ for $12 \mathrm{~h}$. The crude product was 
purified by flash column chromatography on silica gel (cyclohexane/EtOAc 12:1 to 3:1 gradient). The title compound was isolated as a clear, pale light yellow oil (1.07 g, $3.70 \mathrm{mmol}, 92 \%)$.

TLC: $R_{f}=0.30$ (cyclohexane/EtOAc 3:1; UV, CAM); Specific Rotation: $[\alpha]_{\mathrm{D}}^{27}+2.5(\mathrm{c}=1.00$, $\left.\mathrm{CHCl}_{3}\right) ;{ }^{1} \mathbf{H}-\mathbf{N M R}\left(400 \mathrm{MHz}, \mathrm{CDCl}_{3}, 298 \mathrm{~K}\right): \delta 7.30-7.27(\mathrm{~m}, 2 \mathrm{H}), 6.90-6.86(\mathrm{~m}, 2 \mathrm{H}), 4.53(\mathrm{~s}, 2 \mathrm{H})$, $4.23-4.18(\mathrm{~m}, 3 \mathrm{H}), 3.80(\mathrm{~s}, 3 \mathrm{H}), 1.88-1.85(\mathrm{~m}, 3 \mathrm{H}), 1.80-1.76(\mathrm{~m}, 2 \mathrm{H}), 1.71-1.64(\mathrm{~m}, 1 \mathrm{H}), 1.61$ - 1.55 (m, 1H), 1.32 - 1.03 (m, 5H); ${ }^{13} \mathbf{C}-\mathbf{N M R}\left(101 \mathrm{MHz}, \mathrm{CDCl}_{3}, 298 \mathrm{~K}\right): \delta$ 159.5, 129.9, 129.6, 114.0, 86.7, 81.8, 71.3, 67.4, 57.2, 55.4, 44.2, 28.7, 28.3, 26.5, 26.01, 25.99; IR (neat): 3410 (br), 3000 (w), 2924 (m), 2851 (m), 1612 (m), 1586 (w), 1513 (s), 1450 (m), 1385 (w), 1350 (w), 1302 (m), 1247 (s), 1174 (m), 1112 (w), 1070 (s), 1033 (s), 1011 (s), 941 (w), 920 (w), 893 (w), 846 (w), 819 (s), $758(\mathrm{w}), 710(\mathrm{w}), 674(\mathrm{w}), 577(\mathrm{~m}), 516(\mathrm{~m}) \mathrm{cm}^{-1}$; HRMS (EI) $\mathrm{m} / \mathrm{z}$ : exact mass calculated for $\mathrm{C}_{18} \mathrm{H}_{24} \mathrm{O}_{3}[\mathrm{M}]^{+}$288.1720, found 288.1723; SFC (Jasco 2080 Plus, Daicel Chiralcel OJ-H, 90\% CO , $10 \% \mathrm{MeOH}$ at $100 \mathrm{bar}$, flow rate $2.0 \mathrm{~mL} \cdot \mathrm{min}^{-1}, 25^{\circ} \mathrm{C}$, detection $226 \mathrm{~nm}$ ): $98 \%$ ee, $\mathrm{t}_{\mathrm{R}}$ (major enantiomer) $12.9 \mathrm{~min}, \mathrm{t}_{\mathrm{R}}$ (minor enantiomer) $14.4 \mathrm{~min}$.

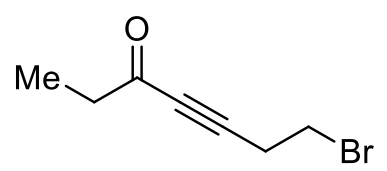

7-Bromohept-4-yn-3-one (SI-4). Following a modified literature procedure, ${ }^{4}$ to a solution of 4-bromo-1-butyne (0.930 mL, $9.91 \mathrm{mmol}, 1.00$ equiv.) in THF (33.0 mL) at $-78{ }^{\circ} \mathrm{C}$ was added $n$-BuLi in hexanes (1.60 M, $6.20 \mathrm{~mL}, 9.92 \mathrm{mmol}, 1.00$ equiv.) over $4 \mathrm{~min}$. The resulting solution was stirred at $-78{ }^{\circ} \mathrm{C}$ for $15 \mathrm{~min}$ before a solution of $N$-methoxy- $N$-methylpropionamide ${ }^{5}(1.16 \mathrm{~g}, 9.90 \mathrm{mmol}$, 1.00 equiv.) in THF (10 mL) was added via cannula at $-78{ }^{\circ} \mathrm{C}$ over $7 \mathrm{~min}$. The cooling bath was removed and the clear, yellow solution was allowed to warm to $0{ }^{\circ} \mathrm{C}$ over $1 \mathrm{~h}$. The brown suspension was quenched at $0{ }^{\circ} \mathrm{C}$ by the addition of sat. aq. $\mathrm{NH}_{4} \mathrm{Cl}(80 \mathrm{~mL})$. Deionized water $(2 \mathrm{~mL})$ was added and the clear solution was transferred into a separation funnel. EtOAc $(60 \mathrm{~mL})$ was used to assist the transfer. The solutions were separated and the aqueous solution was extracted with EtOAc $(60 \mathrm{~mL})$. The EtOAc solutions were washed with sat. aq. $\mathrm{NaCl}(75 \mathrm{~mL})$, dried over $\mathrm{MgSO}_{4}$, filtered, and concentrated under reduced pressure. Purification by flash column chromatography on silica gel

\footnotetext{
${ }^{4}$ Higo, T.; Ukegawa, T.; Yokoshima, S.; Fukuyama, T. Angew. Chem. 2015, 127, 7475.

${ }^{5}$ Kerr, W. J.; Morrison, A. J.; Pazicky, M.; Weber, T. Org. Lett. 2012, 14, 2250. 
(cyclohexane/EtOAc 12:1 to 8:1 gradient) afforded the title compound as a clear, colorless oil (1.06 g, $5.62 \mathrm{mmol}, 57 \%)$.

TLC: $R_{f}=0.24$ (cyclohexane/EtOAc 8:1; UV, $\left.\mathrm{KMnO}_{4}\right) ;{ }^{1} \mathbf{H}-\mathbf{N M R}\left(400 \mathrm{MHz}, \mathrm{CDCl}_{3}, 298 \mathrm{~K}\right)$ : $\delta 3.46(\mathrm{t}, J=7.0 \mathrm{~Hz}, 2 \mathrm{H}), 2.94(\mathrm{t}, J=7.0 \mathrm{~Hz}, 2 \mathrm{H}), 2.57(\mathrm{q}, J=7.4 \mathrm{~Hz}, 2 \mathrm{H}), 1.14(\mathrm{t}, J=7.4 \mathrm{~Hz}, 3 \mathrm{H})$; ${ }^{13}$ C-NMR $\left(101 \mathrm{MHz}, \mathrm{CDCl}_{3}, 298 \mathrm{~K}\right): \delta 188.5,89.5,81.8,38.9,27.9,23.4$, 8.1; IR (film, $\mathrm{CDCl}_{3}$ ): 2979 (w), 2939 (w), 2905 (w), 1672 (s), 1459 (w), 1437 (w), 1409 (w), 1379 (w), 1349 (w), 1330 (w), 1270 (m), 1216 (m), 1174 (s), 1141 (w), 1073 (w), 1023 (m), 991 (w), 934 (m), 897 (w), $842(\mathrm{w})$, $797(\mathrm{w}), 743$ (w), $724(\mathrm{w}), 695$ (w), 670 (w), 632 (w), 561 (m), 518 (w) cm ${ }^{-1}$; HRMS (EI) m/z: exact mass calculated for $\mathrm{C}_{7} \mathrm{H}_{9} \mathrm{BrO}[\mathrm{M}]^{+}$187.9832, found 187.9829 .

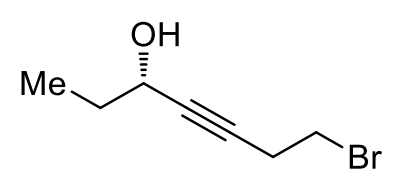

(-)-(S)-7-Bromohept-4-yn-3-ol (SI-5). Following a modified literature procedure, ${ }^{6}$ to a stirred solution of (s)-2-Methyl-CBS-oxazaborolidine (750 mg, $2.71 \mathrm{mmol}, 1.01$ equiv.) in THF (26.0 mL) was added 7-bromohept-4-yn-3-one (508 mg, $2.69 \mathrm{mmol}, 1.00$ equiv.) at $24{ }^{\circ} \mathrm{C}$. The solution was cooled to $-40{ }^{\circ} \mathrm{C}$ and $\mathrm{BH}_{3} \bullet \mathrm{SMe}_{2}(1.50 \mathrm{~mL}, 15.8 \mathrm{mmol}, 5.88$ equiv.) was added dropwise over $10 \mathrm{~min}$. The resulting clear, pale light yellow solution was stirred at $-40^{\circ} \mathrm{C}$ for $2 \mathrm{~h}$ before $\mathrm{MeOH}(5.0 \mathrm{~mL})$ was added. The solution was allowed to warm to $-30{ }^{\circ} \mathrm{C}$ over $1 \mathrm{~h}$ and was then partitioned between sat. aq. $\mathrm{NH}_{4} \mathrm{Cl}(60 \mathrm{~mL})$ and $\mathrm{Et}_{2} \mathrm{O}(120 \mathrm{~mL})$. The phases were separated, the aqueous phase was extracted with $\mathrm{Et}_{2} \mathrm{O}(60 \mathrm{~mL})$, and the combined organic solutions were washed with sat. aq. $\mathrm{NaCl}(60 \mathrm{~mL})$, dried over $\mathrm{MgSO}_{4}$, and concentrated under reduced pressure. Purification by flash column chromatography on silica gel (cyclohexane/EtOAc 8:1 to 4:1 gradient) afforded the title compound as a clear, colorless oil (438 mg, $2.29 \mathrm{mmol}, 85 \%)$.

TLC: $R_{f}=0.30\left(\right.$ cyclohexane/EtOAc 3:1; UV, $\left.\mathrm{KMnO}_{4}\right)$; Specific Rotation: $[\alpha]_{\mathrm{D}}^{23}-6.1(\mathrm{c}=1.00$, $\left.\mathrm{CHCl}_{3}\right) ;{ }^{1} \mathbf{H}-\mathbf{N M R}\left(400 \mathrm{MHz}, \mathrm{CDCl}_{3}, 298 \mathrm{~K}\right): \delta 4.34-4.28(\mathrm{~m}, 1 \mathrm{H}), 3.43(\mathrm{t}, J=7.2 \mathrm{~Hz}, 2 \mathrm{H}), 2.78(\mathrm{td}$, $J=7.2,1.9 \mathrm{~Hz}, 2 \mathrm{H}), 1.83(\mathrm{~d}, J=5.5 \mathrm{~Hz}, 1 \mathrm{H}), 1.76-1.65(\mathrm{~m}, 2 \mathrm{H}), 1.00(\mathrm{t}, J=7.4 \mathrm{~Hz}, 3 \mathrm{H}) ;{ }^{13} \mathrm{C}-\mathrm{NMR}$ (101 MHz, $\left.\mathrm{CDCl}_{3}, 298 \mathrm{~K}\right): \delta$ 83.3, 82.2, 64.0, 31.1, 29.7, 23.3, 9.5; IR (neat): 3339 (br), 2967 (m),

\footnotetext{
${ }^{6}$ Nakayama, A.; Kogure, N.; Kitajima, M.; Takayama, H. Org. Lett. 2009, 11, 5554. 
2934 (m), 2877 (w), 1456 (w), 1434 (w), 1418 (w), 1378 (w), 1333 (w), 1271 (s), 1239 (w), 1212 (s), $1154(w), 1096(w), 1073(w), 1040(\mathrm{~m}), 1006(\mathrm{~s}), 962(\mathrm{~s}), 924(\mathrm{w}), 889(\mathrm{w}), 859(\mathrm{w}), 832(\mathrm{w})$, $814(\mathrm{w}), 749(\mathrm{w}), 702(\mathrm{w}), 659(\mathrm{~m}), 557(\mathrm{~m}) \mathrm{cm}^{-1}$; HRMS (EI) $\mathrm{m} / \mathrm{z}$ : exact mass calculated for $\mathrm{C}_{5} \mathrm{H}_{6} \mathrm{BrO}\left[\mathrm{M}-\mathrm{C}_{2} \mathrm{H}_{5}\right]^{+}$160.9597, found 160.9596 .

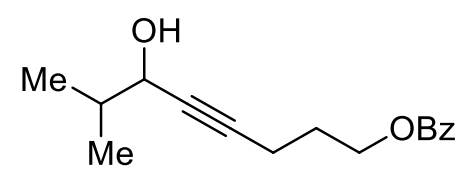

Typical procedure for the synthesis of racemic, secondary propargylic alcohols using LDA. A solution of LDA in THF was prepared by the dropwise addition of $n$-BuLi in hexanes $(1.60 \mathrm{M}$, $3.28 \mathrm{~mL}, 5.28 \mathrm{mmol}, 1.05$ equiv. $)$ to a solution of $i-\mathrm{Pr}_{2} \mathrm{NH}(0.775 \mathrm{~mL}, 1.10$ equiv., $5.53 \mathrm{mmol})$ in THF $(6.45 \mathrm{~mL})$ at $-78{ }^{\circ} \mathrm{C}$. The fresh LDA solution was allowed to stir at $25{ }^{\circ} \mathrm{C}$ for $20 \mathrm{~min}$ and then added via cannula to a solution of pent-4-yn-1-yl benzoate ${ }^{7}$ (946 mg, $5.03 \mathrm{mmol}, 1.00$ equiv.) in THF $(12.5 \mathrm{~mL})$ at $-78{ }^{\circ} \mathrm{C}$. The resulting reaction mixture was stirred at $-78{ }^{\circ} \mathrm{C}$ for $15 \mathrm{~min}$ before isobutyraldehyde $(0.640 \mathrm{~mL}, 7.05 \mathrm{mmol}, 1.40$ equiv. $)$ was added via syringe. The reaction was stirred at $-78{ }^{\circ} \mathrm{C}$ for $30 \mathrm{~min}$ and then quenched at $-78^{\circ} \mathrm{C}$ by the addition of sat. aq. $\mathrm{NH}_{4} \mathrm{Cl}(25 \mathrm{~mL})$. The cooling bath was removed and the mixture was allowed to warm to $25^{\circ} \mathrm{C}$. Deionized water $(2 \mathrm{~mL})$ was added and the clear solution was transferred into a separation funnel. EtOAc $(100 \mathrm{~mL})$ was used to assist the transfer. The solutions were separated and the organic solution was washed with sat. aq. $\mathrm{NaCl}(100 \mathrm{~mL})$, dried over $\mathrm{MgSO}_{4}$, filtered, and concentrated under reduced pressure. Purification by flash column chromatography on silica gel (cyclohexane/EtOAc 8:1 to 3:1 gradient) afforded ( \pm )-6-hydroxy-7-methyloct-4-yn-1-yl benzoate $(1.05 \mathrm{~g}, 4.03 \mathrm{mmol}, 80 \%)$ as a clear, colorless oil. Characterization data were in accordance with (+)-SI-1.

General Procedure B: Benzoylation of Propargylic Alcohols. To a solution of propargylic alcohol (4.0 mmol, 1.0 equiv.) in $\mathrm{CH}_{2} \mathrm{Cl}_{2}(22 \mathrm{~mL}, 0.18 \mathrm{M})$ at $24{ }^{\circ} \mathrm{C}$ was added pyridine $(0.65 \mathrm{~mL}$, $8.0 \mathrm{mmol}, 2.0$ equiv.), DMAP (24 mg, $0.20 \mathrm{mmol}, 0.050$ equiv.) and benzoyl chloride $(0.70 \mathrm{~mL}$, $6.0 \mathrm{mmol}, 1.5$ equiv.). The solution was stirred at $24^{\circ} \mathrm{C}$ for the time indicated for each compound

\footnotetext{
${ }^{7}$ Stevens, B. D.; Nelson, S. G. J. Org. Chem. 2005, 70, 4375.
} 
before $\mathrm{Et}_{2} \mathrm{O}(30 \mathrm{~mL})$ and sat. aq. $\mathrm{NaHCO}_{3}(30 \mathrm{~mL})$ were added. The resulting biphasic mixture was stirred at $24{ }^{\circ} \mathrm{C}$ then transferred to a separation funnel. The transfer was assisted with $\mathrm{Et}_{2} \mathrm{O}(100 \mathrm{~mL})$. The phases were separated, the organic solution was washed with $1.0 \mathrm{M}$ aq. $\mathrm{HCl}(20 \mathrm{~mL})$ and sat. aq. $\mathrm{NaCl}$ (60 $\mathrm{mL}$ ), then dried over $\mathrm{MgSO}_{4}$ and filtered. The solvent was evaporated, and the residue was purified by flash column chromatography on silica gel.

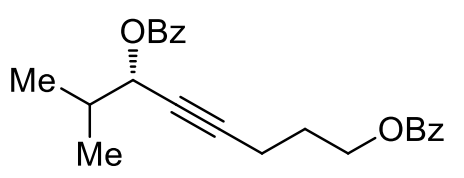

(-)-(S)-7-Methyloct-4-yne-1,6-diyl dibenzoate (1a). General procedure B was followed on a $8.60 \mathrm{mmol}$ scale. The reaction was stirred at $24{ }^{\circ} \mathrm{C}$ for $20 \mathrm{~h}$. The crude product was purified by flash column chromatography on silica gel (cyclohexane/EtOAc 20:1 to 8:1 gradient). The title compound was isolated as a clear, colorless oil (2.90 g, $7.96 \mathrm{mmol}, 92 \%)$.

TLC: $R_{f}=0.34$ (cyclohexane/EtOAc 8:1; UV, CAM); Specific Rotation: $[\alpha]_{\mathrm{D}}^{22}-27.6(\mathrm{c}=1.00$, $\left.\mathrm{CHCl}_{3}\right) ;{ }^{1} \mathbf{H}-\mathbf{N M R}\left(400 \mathrm{MHz}, \mathrm{CDCl}_{3}, 298 \mathrm{~K}\right): \delta 8.09-8.02(\mathrm{~m}, 4 \mathrm{H}), 7.58-7.53(\mathrm{~m}, 2 \mathrm{H}), 7.46-7.41$ $(\mathrm{m}, 4 \mathrm{H}), 5.45(\mathrm{dt}, J=5.5,2.0 \mathrm{~Hz}, 1 \mathrm{H}), 4.41(\mathrm{t}, J=6.3 \mathrm{~Hz}, 2 \mathrm{H}), 2.43(\mathrm{td}, J=7.1,2.0 \mathrm{~Hz}, 2 \mathrm{H}), 2.17-$ $2.08(\mathrm{~m}, 1 \mathrm{H}), 2.00(\operatorname{app~p}, J=6.7 \mathrm{~Hz}, 2 \mathrm{H}), 1.08(\operatorname{app} \mathrm{dd}, J=10.8,6.8 \mathrm{~Hz}, 6 \mathrm{H})$; ${ }^{13} \mathbf{C}-\mathbf{N M R}(101 \mathrm{MHz}$, $\mathrm{CDCl}_{3}, 298 \mathrm{~K}$ ): $\delta 166.6,165.8,133.1,133.1,130.4$ (two coincident resonances), 129.9, 129.7, 128.5 (two coincident resonances), 85.4, 77.3, 70.0, 63.7, 32.9, 28.0, 18.4, 17.9, 15.9; IR (neat): 3063 (w), 2965 (w), 2932 (w), 1717 (s), 1602 (w), 1584 (w), 1491 (w), 1451 (m), 1387 (w), 1361 (w), 1335 (w), $1314(\mathrm{w}), 1264$ (s), $1176(\mathrm{~m}), 1158$ (w), 1107 (s), 1096 (s), 1069 (s), 1026 (m), 973 (m), 936 (w), $915(\mathrm{w}), 805(\mathrm{w}), 750(\mathrm{w}), 708(\mathrm{~s}), 687(\mathrm{~m}), 674(\mathrm{w}), 595(\mathrm{w}) \mathrm{cm}^{-1}$; HRMS (ESI) $\mathrm{m} / z$ : exact mass calculated for $\mathrm{C}_{23} \mathrm{H}_{28} \mathrm{NO}_{4}\left[\mathrm{M}+\mathrm{NH}_{4}\right]^{+}$382.2013, found 382.2011; SFC (Jasco 2080 Plus, Daicel Chiralcel $\mathrm{OJ}-\mathrm{H}, 98 \% \mathrm{CO}_{2}, 2 \% \mathrm{MeOH}$ at $100 \mathrm{bar}$, flow rate $2.0 \mathrm{~mL} \cdot \mathrm{min}^{-1}, 25{ }^{\circ} \mathrm{C}$, detection $225 \mathrm{~nm}$ ): $96 \%$ ee, $\mathrm{t}_{\mathrm{R}}$ (major enantiomer) $14.9 \mathrm{~min}, \mathrm{t}_{\mathrm{R}}$ (minor enantiomer) $16.9 \mathrm{~min}$. 


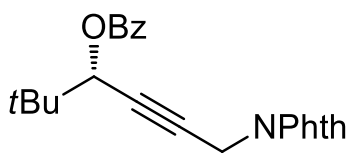

(-)-(S)-6-(1,3-Dioxoisoindolin-2-yl)-2,2-dimethylhex-4-yn-3-yl benzoate (1f). General procedure B was followed on a $4.89 \mathrm{mmol}$ scale. The reaction was stirred at $24{ }^{\circ} \mathrm{C}$ for $18 \mathrm{~h}$. The crude product was purified by recrystallization from EtOAc/n-hexanes. Therefore the crude product was dissolved in $7 \mathrm{~mL}$ of hot EtOAc and $60 \mathrm{~mL} n$-hexanes were added. The solution was allowed to cool to $24{ }^{\circ} \mathrm{C}$ and then placed in a freezer $\left(-20^{\circ} \mathrm{C}\right)$ for $15 \mathrm{~h}$. The title compound was isolated as a white solid (1.68 g, $4.46 \mathrm{mmol}, 91 \%)$ in one crop.

TLC: $\quad R_{f}=0.36$ (cyclohexane/EtOAc 3:1; UV, CAM); Melting point: $123-125^{\circ} \mathrm{C}$ (EtOAc/n-hexane); Specific Rotation: $[\alpha]_{\mathrm{D}}^{22}-39.2$ (c = 1.00, $\mathrm{CHCl}_{3}$ ); ${ }^{1} \mathbf{H}-\mathbf{N M R}\left(400 \mathrm{MHz}, \mathrm{CDCl}_{3}\right.$, $298 \mathrm{~K}): \delta 8.09-8.06(\mathrm{~m}, 2 \mathrm{H}), 7.92-7.87(\mathrm{~m}, 2 \mathrm{H}), 7.78-7.73(\mathrm{~m}, 2 \mathrm{H}), 7.61-7.56(\mathrm{~m}, 1 \mathrm{H}), 7.48-$ $7.44(\mathrm{~m}, 2 \mathrm{H}), 5.36(\mathrm{t}, J=1.8 \mathrm{~Hz}, 1 \mathrm{H}), 4.52(\mathrm{~d}, J=1.8 \mathrm{~Hz}, 2 \mathrm{H}), 1.09$ (s, 9H); ${ }^{13} \mathbf{C}-\mathbf{N M R}(101 \mathrm{MHz}$, $\left.\mathrm{CDCl}_{3}, 298 \mathrm{~K}\right): \delta 167.1,165.6,134.3,133.2,132.1,130.1,129.9,128.5,123.7,79.7,79.6,72.2,35.8$, 27.5, 25.8; IR (neat): 2979 (w), 2963 (w), 1771 (m), 1713 (s), 1618 (w), $1599(\mathrm{w}), 1474(\mathrm{~m})$, 1449 (w), 1423 (m), 1394 (m), 1367 (w), 1339 (m), 1322 (m), 1299 (m), 1259 (s), 1248 (m), 1188 (w), 1175 (w), 1141 (m), 1121 (m), 1105 (s), 1095 (m), 1067 (m), 1024 (m), 972 (m), 944 (m), 923 (w), 793 (w), 727 (w), 706 (s), 685 (m), 633 (m), 570 (w), 530 (m), 471 (w) cm ${ }^{-1}$; HRMS (ESI) m/z: exact mass calculated for $\mathrm{C}_{23} \mathrm{H}_{25} \mathrm{~N}_{2} \mathrm{O}_{4}\left[\mathrm{M}+\mathrm{NH}_{4}\right]^{+}$393.1809, found 393.1812; SFC (Waters Acquity UPC ${ }^{2}$, Trefoil CEL2, $85 \% \mathrm{CO}_{2}, 15 \% \mathrm{MeOH}$, flow rate $2.0 \mathrm{~mL} \cdot \mathrm{min}^{-1}, 40{ }^{\circ} \mathrm{C}$, detection $254 \mathrm{~nm}$ ): $97 \%$ ee, $\mathrm{t}_{\mathrm{R}}$ (major enantiomer) $1.1 \mathrm{~min}, \mathrm{t}_{\mathrm{R}}$ (minor enantiomer) $1.5 \mathrm{~min}$.

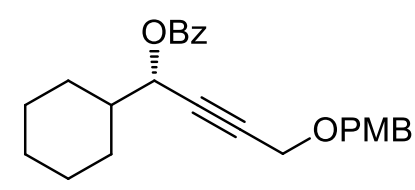

(-)-(S)-1-Cyclohexyl-4-((4-methoxybenzyl)oxy)but-2-yn-1-yl benzoate (1i). General procedure B was followed on a $3.57 \mathrm{mmol}$ scale. The reaction was stirred at $24{ }^{\circ} \mathrm{C}$ for $18 \mathrm{~h}$. The crude product was purified by flash column chromatography on silica gel (pentane/ $\mathrm{Et}_{2} \mathrm{O} 8: 1$ to 5:1 gradient). The title compound was isolated as a clear, colorless oil (1.22 g, $3.10 \mathrm{mmol}, 87 \%)$. The enantiomeric 
excess was determined for the starting material ((+)-(S)-1-cyclohexyl-4-((4-methoxybenzyl)oxy)but2-yn-1-ol (SI-3), 98\% ee).

TLC: $R_{f}=0.33$ (pentane/Et $2 \mathrm{O} 5: 1$; UV, CAM); Specific Rotation: $[\alpha]_{\mathrm{D}}^{22}-32.6\left(\mathrm{c}=1.00, \mathrm{CHCl}_{3}\right)$; ${ }^{1} \mathbf{H}-\mathbf{N M R}\left(400 \mathrm{MHz}, \mathrm{CDCl}_{3}, 298 \mathrm{~K}\right): \delta 8.12-8.10$ (m, 2H), $7.62-7.58$ (m, 1H), 7.50 - 7.46 (m, 2H), $7.31-7.27(\mathrm{~m}, 2 \mathrm{H}), 6.90-6.86(\mathrm{~m}, 2 \mathrm{H}), 5.56(\mathrm{dt}, J=5.9,1.6 \mathrm{~Hz}, 1 \mathrm{H}), 4.56(\mathrm{~s}, 2 \mathrm{H}), 4.21(\mathrm{~d}$, $J=1.7 \mathrm{~Hz}, 2 \mathrm{H}), 3.82(\mathrm{~s}, 3 \mathrm{H}), 2.01-1.81(\mathrm{~m}, 5 \mathrm{H}), 1.75-1.72(\mathrm{~m}, 1 \mathrm{H}), 1.38-1.17(\mathrm{~m}, 5 \mathrm{H}) ;{ }^{13} \mathbf{C}-\mathbf{N M R}$ (101 MHz, $\left.\mathrm{CDCl}_{3}, 298 \mathrm{~K}\right): \delta 165.7,159.5,133.2,130.2,130.0,129.9,129.5,128.5,114.0,83.3,82.4$, 71.2, 69.0, 57.1, 55.4, 42.2, 28.8, 28.5, 26.4, 25.94, 25.89; IR (neat): 2929 (m), 2853 (w), 1719 (s), $1612(\mathrm{w}), 1585$ (w), 1513 (m), 1451 (m), 1347 (w), 1315 (w), 1248 (s), 1175 (m), 1155 (w), 1106 (m), $1096(\mathrm{~m}), 1068(\mathrm{~s}), 1035(\mathrm{~m}), 1026(\mathrm{~m}), 971(\mathrm{~m}), 950(\mathrm{w}), 891(\mathrm{w}), 846(\mathrm{w}), 819(\mathrm{~m}), 758(\mathrm{w})$, $710(\mathrm{~s}), 688(\mathrm{w}), 661(\mathrm{w}), 576(\mathrm{w}), 517(\mathrm{w}) \mathrm{cm}^{-1}$; HRMS (ESI) $\mathrm{m} / \mathrm{z}$ : exact mass calculated for $\mathrm{C}_{25} \mathrm{H}_{32} \mathrm{NO}_{4}\left[\mathrm{M}+\mathrm{NH}_{4}\right]^{+} 410.2326$, found 410.2335 .

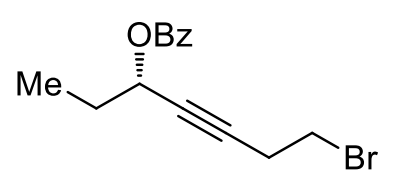

(-)-(S)-7-Bromohept-4-yn-3-yl benzoate (1k). General procedure B was followed on a $2.10 \mathrm{mmol}$ scale. The reaction was stirred at $24^{\circ} \mathrm{C}$ for $11 \mathrm{~h}$. The crude product was purified by flash column chromatography on silica gel (cyclohexane/EtOAc 20:1). The title compound was isolated as a clear, colorless oil (556 mg, $1.89 \mathrm{mmol}, 90 \%)$.

TLC: $R_{f}=0.25$ (cyclohexane/EtOAc 12:1; UV, CAM); Specific Rotation: $[\alpha]_{\mathrm{D}}^{21}-26.2(\mathrm{c}=1.00$, $\left.\mathrm{CHCl}_{3}\right) ;{ }^{1} \mathbf{H}-\mathbf{N M R}\left(400 \mathrm{MHz}, \mathrm{CDCl}_{3}, 298 \mathrm{~K}\right): \delta 8.08-8.06(\mathrm{~m}, 2 \mathrm{H}), 7.59-7.55(\mathrm{~m}, 1 \mathrm{H}), 7.47-7.43$ $(\mathrm{m}, 2 \mathrm{H}), 5.55(\mathrm{tt}, J=6.4,1.9 \mathrm{~Hz}, 1 \mathrm{H}), 3.43(\mathrm{t}, J=7.3 \mathrm{~Hz}, 2 \mathrm{H}), 2.79(\mathrm{td}, J=7.3,1.9 \mathrm{~Hz}, 2 \mathrm{H}), 1.95-$ $1.88(\mathrm{~m}, 2 \mathrm{H}), 1.09$ (t, $J=7.4 \mathrm{~Hz}, 3 \mathrm{H}) ;{ }^{13} \mathrm{C}-\mathbf{N M R}\left(101 \mathrm{MHz}, \mathrm{CDCl}_{3}, 298 \mathrm{~K}\right): \delta 165.7,133.2,130.2$, 129.9, 128.5, 82.9, 79.8, 66.0, 29.4, 28.4, 23.4, 9.6; IR (neat): 2972 (w), 2937 (w), 2879 (w), 1718 (s), 1601 (w), 1585 (w), 1491 (w), 1451 (m), 1382 (w), 1341 (w), 1314 (w), 1299 (m), 1264 (s), 1213 (m), $1176(\mathrm{w}), 1176$ (w), 1167 (w), 1106 (m), 1069 (m), 1042 (w), 1025 (m), 999 (w), 959 (w), 927 (m), 896 (w), 804 (w), 709 (s), 687 (m), 674 (w), 591 (w), 568 (w) cm ${ }^{-1}$; HRMS (ESI) m/z: exact mass calculated for $\mathrm{C}_{14} \mathrm{H}_{15} \mathrm{BrNaO}_{2}[\mathrm{M}+\mathrm{Na}]^{+}$317.0148, found 317.0153; SFC (Jasco 2080 Plus, Daicel 
Chiralcel $\mathrm{OB}-\mathrm{H}, 99 \% \mathrm{CO}_{2}, 1 \% \mathrm{MeOH}$ at $100 \mathrm{bar}$, flow rate $2.0 \mathrm{~mL} \cdot \mathrm{min}^{-1}, 25{ }^{\circ} \mathrm{C}$, detection $215 \mathrm{~nm}$ ): $86 \%$ ee, $\mathrm{t}_{\mathrm{R}}$ (minor enantiomer) $7.4 \mathrm{~min}, \mathrm{t}_{\mathrm{R}}$ (major enantiomer) $8.6 \mathrm{~min}$.

\section{Synthesis and Characterization of Products}

\section{General procedure C: Stereoselective synthesis of allenes from propargylic benzoates and} arylboronic acids. To a non-dried two necked $25 \mathrm{~mL}$ round bottomed flask equipped with a stir bar was added the propargylic benzoate $(0.500 \mathrm{mmol}, 1.00$ equiv.). The side neck was connected to a gas inlet adapter and the flask was purged with $\mathrm{N}_{2}$ for $5 \mathrm{~min}$, then the top neck sealed with a glass stopper. To the flask were subsequently added 1,2-dichloroethane $(2.50 \mathrm{~mL})$, the corresponding arylboronic acid (1.00 mmol, 2.00 equiv.), and $\mathrm{K}_{3} \mathrm{PO}_{4}\left(212 \mathrm{mg}, 1.00 \mathrm{mmol}, 2.00\right.$ equiv.) against a flow of $\mathrm{N}_{2}$ (while replacing the glass stopper). Finally a clear, red solution of $\left[\{\mathrm{Rh}(\operatorname{cod}) \mathrm{Cl}\}_{2}\right](7.4 \mathrm{mg}$, $0.015 \mathrm{mmol}, \quad 3.0 \mathrm{~mol} \%)$ and phosphoramidite $4(25.5 \mathrm{mg}, \quad 0.0625 \mathrm{mmol}, \quad 12.5 \mathrm{~mol} \%)$ in 1,2-dichloroethane $(1.00 \mathrm{~mL})$ - prepared in a screw-capped vial, sparged with $\mathrm{N}_{2}$ for 1 min and stirred at $24{ }^{\circ} \mathrm{C}$ for $30 \mathrm{~min}$ prior to the addition - was added via Pasteur pipette against a flow of $\mathrm{N}_{2}$. The flask was sealed and immersed in an oil bath. The orange heterogeneous mixture was stirred at $50{ }^{\circ} \mathrm{C}$ for $24 \mathrm{~h}$. The mixture was allowed to cool to $24^{\circ} \mathrm{C}$ and partitioned between $\mathrm{Et}_{2} \mathrm{O}(100 \mathrm{~mL})$ and sat. aq. $\mathrm{NaHCO}_{3}(50 \mathrm{~mL})$. The aqueous layer was extracted with $\mathrm{Et}_{2} \mathrm{O}(50 \mathrm{~mL})$. The combined organic layers were washed with sat. aq. $\mathrm{NaCl}(50 \mathrm{~mL})$, dried over $\mathrm{MgSO}_{4}$, and filtered. The solution was concentrated and the residue was purified by flash column chromatography on silica gel. All deviations from the general procedure, and the solvent system for chromatography are given below for each substrate.

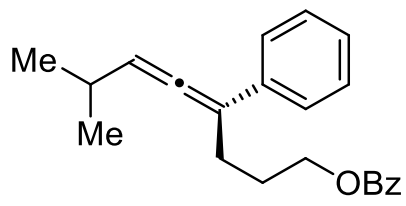

(+)-(S)-7-Methyl-4-phenylocta-4,5-dien-1-yl benzoate (3a). General procedure C was followed. (-)-(S)-7-methyloct-4-yne-1,6-diyl dibenzoate (1a) (182 mg, $0.500 \mathrm{mmol}, 96 \%$ ee ) and phenylboronic acid (122 mg, $1.00 \mathrm{mmol}$ ) were used. The crude product was purified by flash column chromatography 
on silica gel (cyclohexane/EtOAc 50:1 to 20:1 gradient). The title compound was isolated as a light yellow, clear oil (132 mg, $0.412 \mathrm{mmol}, 82 \%)$.

TLC: $R_{f}=0.25$ (cyclohexane/EtOAc 20:1; UV, CAM); Specific Rotation: $[\alpha]_{\mathrm{D}}^{22}+75.6(\mathrm{c}=1.00$, $\left.\mathrm{CHCl}_{3}\right) ;{ }^{1} \mathbf{H}$-NMR $\left(400 \mathrm{MHz}, \mathrm{CDCl}_{3}, 298 \mathrm{~K}\right): \delta 8.08-8.05(\mathrm{~m}, 2 \mathrm{H}), 7.58-7.54(\mathrm{~m}, 1 \mathrm{H}), 7.47-7.42$ (m, 4H), $7.34-7.29(\mathrm{~m}, 2 \mathrm{H}), 7.22-7.18(\mathrm{~m}, 1 \mathrm{H}), 5.60-5.57(\mathrm{~m}, 1 \mathrm{H}), 4.43$ (app td, J = 6.4, $1.5 \mathrm{~Hz}$, 2H), $2.63-2.58(\mathrm{~m}, 2 \mathrm{H}), 2.50-2.39(\mathrm{~m}, 1 \mathrm{H}), 2.08-2.00(\mathrm{~m}, 2 \mathrm{H}), 1.09(\mathrm{~d}, J=6.8 \mathrm{~Hz}, 6 \mathrm{H}) ;{ }^{13} \mathbf{C}-\mathbf{N M R}$ $\left(101 \mathrm{MHz}, \mathrm{CDCl}_{3}, 298 \mathrm{~K}\right): \delta 202.1,166.8,137.3,133.0,130.6,129.7,128.5,128.5,126.7,125.8$, 106.0, 102.8, 64.8, 28.9, 27.4, 26.6, 22.9; IR (neat): 3061 (w), 2958 (m), $2925(w), 2868(w)$, 1946 (w), 1717 (s), 1600 (w), 1493 (m), 1451 (m), 1381 (w), 1363 (w), 1314 (w), 1270 (s), 1176 (m), 1113 (s), 1070 (m), 1027 (m), 977 (w), 935 (w), 813 (w), 754 (m), 710 (s), 693 (s), 676 (w), 648 (w), 617 (w) $\mathrm{cm}^{-1}$; HRMS (ESI) $m / z$ : exact mass calculated for $\mathrm{C}_{22} \mathrm{H}_{24} \mathrm{NaO}_{2}[\mathrm{M}+\mathrm{Na}]^{+} 343.1669$, found 343.1671; SFC (Jasco 2080 Plus, Daicel Chiralpak IB, 99\% $\mathrm{CO}_{2}, 1 \% \mathrm{MeOH}$ at 100 bar, flow rate $2.0 \mathrm{~mL} \cdot \mathrm{min}^{-1}, 25{ }^{\circ} \mathrm{C}$, detection $253 \mathrm{~nm}$ ): $94 \%$ ee, $\mathrm{t}_{\mathrm{R}}$ (minor enantiomer) $15.0 \mathrm{~min}$, $\mathrm{t}_{\mathrm{R}}$ (major enantiomer) $15.8 \mathrm{~min}$.

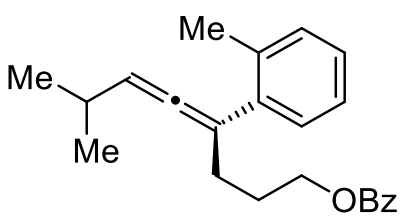

(+)-(S)-7-Methyl-4-(o-tolyl)octa-4,5-dien-1-yl benzoate (3b). General procedure C was followed but the reaction was stirred at $40{ }^{\circ} \mathrm{C}$ for $12 \mathrm{~h}$. (-)-(S)-7-methyloct-4-yne-1,6-diyl dibenzoate (1a) (182 mg, $0.500 \mathrm{mmol}, 96 \%$ ee ) and 2-methylphenylboronic acid (136 mg, $1.00 \mathrm{mmol}$ ) were used. The crude product was purified by flash column chromatography on silica gel (cyclohexane/EtOAc 20:1). The title compound was isolated as a light yellow, clear oil (153 mg, $0.457 \mathrm{mmol}, 91 \%)$.

TLC: $R_{f}=0.33$ (cyclohexane/EtOAc 20:1; UV, CAM); Specific Rotation: $[\alpha]_{\mathrm{D}}^{28}+40.7(\mathrm{c}=1.00$, $\left.\mathrm{CHCl}_{3}\right) ;{ }^{1} \mathbf{H}-\mathbf{N M R}\left(400 \mathrm{MHz}, \mathrm{CDCl}_{3}, 298 \mathrm{~K}\right): \delta 8.06-8.03(\mathrm{~m}, 2 \mathrm{H}), 7.58-7.54(\mathrm{~m}, 1 \mathrm{H}), 7.46-7.42$ $(\mathrm{m}, 2 \mathrm{H}), 7.23-7.15(\mathrm{~m}, 4 \mathrm{H}), 5.28-5.25(\mathrm{~m}, 1 \mathrm{H}), 4.43-4.37(\mathrm{~m}, 2 \mathrm{H}), 2.49-2.44(\mathrm{~m}, 2 \mathrm{H}), 2.40-$ $2.32(\mathrm{~m}, 4 \mathrm{H}), 2.00-1.93(\mathrm{~m}, 2 \mathrm{H}), 1.04(\mathrm{app} \mathrm{dd}, J=6.8,0.8 \mathrm{~Hz}, 6 \mathrm{H}) ;{ }^{13} \mathbf{C}-\mathbf{N M R}\left(101 \mathrm{MHz}, \mathrm{CDCl}_{3}\right.$, $298 \mathrm{~K}): \delta 200.8,166.8,138.5,135.9,133.0,130.6$ (two coincident resonances), 129.7, 128.5, 128.3, 126.9, 125.9, 105.0, 99.9, 64.7, 30.5, 28.6, 27.2, 22.9, 22.8, 20.5; IR (neat): $3063(\mathrm{w}), 2957$ (m), 
2925 (w), 2868 (w), 1958 (w), 1718 (s), 1602 (w), 1585 (w), 1489 (w), 1451 (m), 1380 (w), 1363 (w), $1314(\mathrm{w}), 1270(\mathrm{~s}), 1176(\mathrm{w}), 1112(\mathrm{~m}), 1070(\mathrm{w}), 1027(\mathrm{w}), 975(\mathrm{w}), 936(\mathrm{w}), 806(\mathrm{w}), 756(\mathrm{w})$, 710 (s), 675 (w) $\mathrm{cm}^{-1}$; HRMS (ESI) $m / z$ : exact mass calculated for $\mathrm{C}_{23} \mathrm{H}_{26} \mathrm{NaO}_{2}[\mathrm{M}+\mathrm{Na}]^{+}$357.1825, found 357.1825; SFC (Jasco 2080 Plus, Daicel Chiralpak IB, 98\% CO2, 2\% $\mathrm{MeOH}$ at 100 bar, flow rate $2.0 \mathrm{~mL} \cdot \mathrm{min}^{-1}, 25^{\circ} \mathrm{C}$, detection $209 \mathrm{~nm}$ ): $93 \%$ ee, $\mathrm{t}_{\mathrm{R}}$ (minor enantiomer) $9.0 \mathrm{~min}, \mathrm{t}_{\mathrm{R}}$ (major enantiomer) $10.3 \mathrm{~min}$.

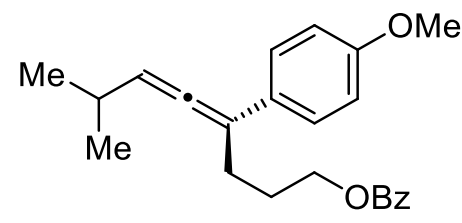

(+)-(S)-4-(4-Methoxyphenyl)-7-methylocta-4,5-dien-1-yl benzoate (3c). General procedure C was followed but the reaction was stirred at $40{ }^{\circ} \mathrm{C}$ for $12 \mathrm{~h}$. (-)-(S)-7-methyloct-4-yne-1,6-diyl dibenzoate (1a) (182 mg, $0.500 \mathrm{mmol}, 96 \%$ ee) and 4-methoxyphenylboronic acid (152 mg, $1.00 \mathrm{mmol}$ ) were used. The crude product was purified by flash column chromatography on silica gel (cyclohexane/EtOAc 20:1). The title compound was isolated as a light yellow, clear oil (150 mg, $0.427 \mathrm{mmol}, 85 \%)$.

TLC: $R_{f}=0.30$ (cyclohexane/EtOAc 12:1; UV, CAM); Specific Rotation: $[\alpha]_{\mathrm{D}}^{28}+77.2(\mathrm{c}=1.00$, $\left.\mathrm{CHCl}_{3}\right) ;{ }^{1} \mathbf{H}-\mathbf{N M R}\left(400 \mathrm{MHz}, \mathrm{CDCl}_{3}, 298 \mathrm{~K}\right): \delta 8.07$ - $8.05(\mathrm{~m}, 2 \mathrm{H}), 7.58-7.54(\mathrm{~m}, 1 \mathrm{H}), 7.46-7.42$ (m, 2H), $7.37-7.34(\mathrm{~m}, 2 \mathrm{H}), 6.88-6.85(\mathrm{~m}, 2 \mathrm{H}), 5.57-5.54(\mathrm{~m}, 1 \mathrm{H}), 4.45-4.39(\mathrm{~m}, 2 \mathrm{H}), 3.80(\mathrm{~s}$, 3H), $2.59-2.55(\mathrm{~m}, 2 \mathrm{H}), 2.47-2.39(\mathrm{~m}, 1 \mathrm{H}), 2.06-1.99(\mathrm{~m}, 2 \mathrm{H}), 1.08(\mathrm{~d}, J=6.8 \mathrm{~Hz}, 6 \mathrm{H}) ;{ }^{13} \mathbf{C}-\mathbf{N M R}$

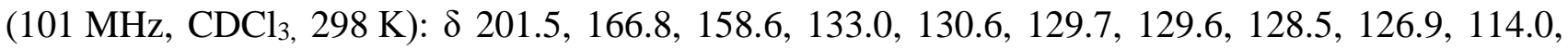
105.5, 102.7, 64.8, 55.4, 28.9, 27.3, 26.8, 22.9; IR (neat): 2957 (m), 2867 (w), 2836 (w), 1717 (s), 1605 (m), 1589 (w), 1509 (s), 1451 (m), 1381 (w), 1363 (w), 1314 (w), 1271 (s), 1246 (s), 1176 (s), 1112 (s), 1070 (w), 1027 (m), 978 (w), 833 (m), 805 (w), 710 (s), 687 8w), 594 (w) cm c $^{-1}$ HRMS (ESI) $m / z$ : exact mass calculated for $\mathrm{C}_{23} \mathrm{H}_{26} \mathrm{NaO}_{3}[\mathrm{M}+\mathrm{Na}]^{+} 373.1774$, found 373.1770; SFC (Jasco 2080 Plus, Daicel Chiralpak IB, 98\% $\mathrm{CO}_{2}, 2 \% \mathrm{MeOH}$ at $100 \mathrm{bar}$, flow rate $2.0 \mathrm{~mL} \cdot \mathrm{min}^{-1}, 25^{\circ} \mathrm{C}$, detection $253 \mathrm{~nm}$ ): 94\% ee, $\mathrm{t}_{\mathrm{R}}$ (minor enantiomer) $15.7 \mathrm{~min}, \mathrm{t}_{\mathrm{R}}$ (major enantiomer) $17.1 \mathrm{~min}$. 


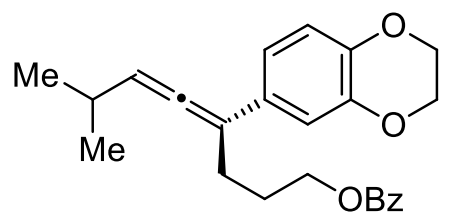

(+)-(S)-4-(2,3-Dihydrobenzo[b][1,4]dioxin-6-yl)-7-methylocta-4,5-dien-1-yl benzoate (3d).

General procedure C was followed. (-)-(S)-7-methyloct-4-yne-1,6-diyl dibenzoate (1a) (182 mg, $0.500 \mathrm{mmol}, 96 \%$ ee $)$ and 2,3-dihydrobenzo[b][1,4]dioxin-6-ylboronic acid (180 $\mathrm{mg}, 1.00 \mathrm{mmol})$ were used. The crude product was purified by flash column chromatography on silica gel (cyclohexane/EtOAc 12:1). The title compound was isolated as a light yellow, clear oil (168 mg, $0.443 \mathrm{mmol}, 89 \%)$.

TLC: $R_{f}=0.35$ (cyclohexane/EtOAc 5:1; UV, CAM); Specific Rotation: $[\alpha]_{\mathrm{D}}^{22}+39.4(\mathrm{c}=1.00$, $\left.\mathrm{CHCl}_{3}\right) ;{ }^{1} \mathbf{H}-\mathbf{N M R}\left(400 \mathrm{MHz}, \mathrm{CDCl}_{3}, 298 \mathrm{~K}\right): \delta 8.07-8.05(\mathrm{~m}, 2 \mathrm{H}), 7.58-7.53(\mathrm{~m}, 1 \mathrm{H}), 7.46-7.42$ $(\mathrm{m}, 2 \mathrm{H}), 6.95-6.91(\mathrm{~m}, 2 \mathrm{H}), 6.81(\mathrm{~d}, J=8.3 \mathrm{~Hz}, 1 \mathrm{H}), 5.56-5.53(\mathrm{~m}, 1 \mathrm{H}), 4.41$ (app td, $J=6.4$, $1.6 \mathrm{~Hz}, 2 \mathrm{H}), 4.25(\mathrm{~s}, 4 \mathrm{H}), 2.56-2.51(\mathrm{~m}, 2 \mathrm{H}), 2.46-2.36(\mathrm{~m}, 1 \mathrm{H}), 2.01-1.98(\mathrm{~m}, 2 \mathrm{H}), 1.07(\mathrm{~d}$, $J=6.8 \mathrm{~Hz}, 6 \mathrm{H}) ;{ }^{13} \mathrm{C}-\mathrm{NMR}\left(101 \mathrm{MHz}, \mathrm{CDCl}_{3}, 298 \mathrm{~K}\right): \delta 201.6,166.8,143.5,142.5,133.0,130.8$, 130.6, 129.7, 128.5, 119.1, 117.2, 114.6, 105.4, 102.8, 64.8, 64.6, 64.5, 28.9, 27.3, 26.7, 22.8, 22.8; IR (neat): 3062 (w), 2958 (w), 2871 (w), 1945 (w), 1715 (s), 1602 (w), 1583 (m), 1504 (s), 1451 (m), 1430 (w), 1382 (w), 1304 (m), 1272 (s), 1175 (m), 1114 (s), 1068 (s), 1027 (m), 1001 (w), 930 (w), $891(\mathrm{~m}), 873$ (w), 811 (m), 749 (w), 711 (s), 687 (w), 675 (w), 631 (w), 602 (w) cm ${ }^{-1}$; HRMS (ESI) $m / z$ : exact mass calculated for $\mathrm{C}_{24} \mathrm{H}_{27} \mathrm{O}_{4}[\mathrm{M}+\mathrm{H}]^{+}$379.1904, found 379.1907; SFC (Jasco 2080 Plus, Daicel Chiralpak IB, 95\% $\mathrm{CO}_{2}, 5 \% \mathrm{MeOH}$ at $100 \mathrm{bar}$, flow rate $2.0 \mathrm{~mL} \cdot \mathrm{min}^{-1}, 25{ }^{\circ} \mathrm{C}$, detection $271 \mathrm{~nm}$ ): $94 \%$ ee, $\mathrm{t}_{\mathrm{R}}$ (major enantiomer) $21.7 \mathrm{~min}, \mathrm{t}_{\mathrm{R}}$ (minor enantiomer) $23.8 \mathrm{~min}$.

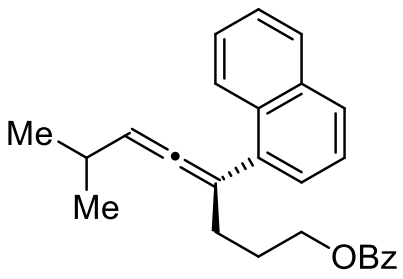

(+)-(S)-7-Methyl-4-(naphthalen-1-yl)octa-4,5-dien-1-yl benzoate (3e). General procedure C was followed. (-)-(S)-7-methyloct-4-yne-1,6-diyl dibenzoate (1a) (182 mg, $0.500 \mathrm{mmol}, 96 \%$ ee) and 1-naphthaleneboronic acid $(172 \mathrm{mg}, 1.00 \mathrm{mmol})$ were used. The crude product was purified by flash S-19 
column chromatography on silica gel (cyclohexane/EtOAc 50:1 to 20:1 gradient). The title compound was isolated as a yellow, clear oil (176 mg, $0.476 \mathrm{mmol}, 95 \%)$.

TLC: $R_{f}=0.25$ (cyclohexane/EtOAc 20:1; UV, CAM); Specific Rotation: $[\alpha]_{\mathrm{D}}^{22}+54.7(\mathrm{c}=1.00$, $\left.\mathrm{CHCl}_{3}\right) ;{ }^{1} \mathbf{H}-\mathbf{N M R}\left(400 \mathrm{MHz}, \mathrm{CDCl}_{3}, 298 \mathrm{~K}\right): \delta 8.19-8.15(\mathrm{~m}, 1 \mathrm{H}), 8.05-8.02(\mathrm{~m}, 2 \mathrm{H}), 7.88-7.84$ (m, 1H), $7.77(\mathrm{~d}, J=7.8 \mathrm{~Hz}, 1 \mathrm{H}), 7.58-7.53(\mathrm{~m}, 1 \mathrm{H}), 7.51-7.40(\mathrm{~m}, 6 \mathrm{H}), 5.38-5.35(\mathrm{~m}, 1 \mathrm{H}), 4.44$ $(\operatorname{app} \mathrm{td}, J=6.5,0.9 \mathrm{~Hz}, 2 \mathrm{H}), 2.65-2.61(\mathrm{~m}, 2 \mathrm{H}), 2.47-2.35(\mathrm{~m}, 1 \mathrm{H}), 2.06-1.99(\mathrm{~m}, 2 \mathrm{H}), 1.07$ (app dd, $J=6.8,2.8 \mathrm{~Hz}, 6 \mathrm{H}) ;{ }^{13} \mathbf{C}-\mathbf{N M R}\left(101 \mathrm{MHz}, \mathrm{CDCl}_{3}, 298 \mathrm{~K}\right): \delta$ 201.5, 166.8, 137.1, 134.1, 133.0, $131.4,130.5,129.7,128.5,128.4,127.5,125.9,125.8,125.7,125.7,125.6,104.3,99.9,64.6,31.3$, 28.6, 27.4, 22.9, 22.7; IR (neat): 3060 (w), 2957 (w), 2925 (w), 2867 (w), 1957 (w), 1717 (s), 1602 (w), 1583 (w), 1506 (w), 1492 (w), 1451 (w), 1392 (w), 1363 (w), 1314 (w), 1270 (s), 1175 (w), $1112(\mathrm{~m}), 1070$ (m), 1027 (m), 968 (w), 933 (w), 862 (w), 800 (m), 777 (s), 735 (w), 710 (s), 687 (w), $675(\mathrm{w}), 635(\mathrm{w}), 526(\mathrm{w}) \mathrm{cm}^{-1}$; HRMS (ESI) $\mathrm{m} / z$ : exact mass calculated for $\mathrm{C}_{26} \mathrm{H}_{26} \mathrm{NaO}_{2}[\mathrm{M}+\mathrm{H}]^{+}$ 393.1825, found 393.1828; SFC (Jasco 2080 Plus, Daicel Chiralpak IB, 90\% CO $2,10 \% \mathrm{MeOH}$ at $100 \mathrm{bar}$, flow rate $2.0 \mathrm{~mL} \cdot \mathrm{min}^{-1}, 25^{\circ} \mathrm{C}$, detection $225 \mathrm{~nm}$ ): $93 \%$ ee, $\mathrm{t}_{\mathrm{R}}$ (major enantiomer) $11.1 \mathrm{~min}$, $\mathrm{t}_{\mathrm{R}}$ (minor enantiomer) $14.5 \mathrm{~min}$.

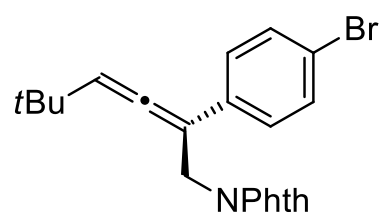

(+)-(R)-2-(2-(4-Bromophenyl)-5,5-dimethylhexa-2,3-dien-1-yl)isoindoline-1,3-dione

(3f).

General procedure $\mathrm{C}$ was followed but deionized water $(90.0 \mu \mathrm{L}, 5.00 \mathrm{mmol}, 10.0$ equiv.) was added subsequent to the addition of $\mathrm{K}_{3} \mathrm{PO}_{4}$. (-)-(S)-6-(1,3-dioxoisoindolin-2-yl)-2,2-dimethylhex-4-yn-3-yl benzoate (1f) (188 mg, $0.500 \mathrm{mmol}, 97 \% \mathrm{ee})$ and 4-bromophenylboronic acid (201 mg, $1.00 \mathrm{mmol})$ were used. The crude product was purified by flash column chromatography on silica gel (cyclohexane/EtOAc 12:1 to 8:1 gradient). The title compound was isolated as a light yellow solid (185 mg, $0.451 \mathrm{mmol}, 90 \%)$. Crystals suitable for X-ray crystallographic analysis were obtained by slow evaporation from $\mathrm{CH}_{2} \mathrm{Cl}_{2} / n$-hexane at $24{ }^{\circ} \mathrm{C}$.

TLC: $R_{f}=0.32$ (cyclohexane/EtOAc 5:1; UV, CAM); Melting point: $126-128{ }^{\circ} \mathrm{C}$ $\left(\mathrm{CH}_{2} \mathrm{Cl}_{2} / n\right.$-hexane); Specific Rotation: $[\alpha]_{\mathrm{D}}^{22}+85.8$ (c = 1.00, $\left.\mathrm{CHCl}_{3}\right) ;{ }^{1} \mathbf{H}-\mathbf{N M R}\left(400 \mathrm{MHz}, \mathrm{CDCl}_{3}\right.$, 
$298 \mathrm{~K}): \delta 7.89-7.84(\mathrm{~m}, 2 \mathrm{H}), 7.74-7.70(\mathrm{~m}, 2 \mathrm{H}), 7.47-7.43(\mathrm{~m}, 2 \mathrm{H}), 7.29-7.26(\mathrm{~m}, 2 \mathrm{H}), 5.51(\mathrm{t}$, $J=3.6 \mathrm{~Hz}, 1 \mathrm{H}), 4.73(\mathrm{dd}, J=15.4,3.5 \mathrm{~Hz}, 1 \mathrm{H}), 4.61(\mathrm{dd}, J=15.4,3.8 \mathrm{~Hz}, 1 \mathrm{H}), 0.84(\mathrm{~s}, 9 \mathrm{H})$; ${ }^{13}$ C-NMR (101 MHz, $\left.\mathrm{CDCl}_{3}, 298 \mathrm{~K}\right): \delta$ 199.4, 167.9, 134.2, 134.1, 132.3, 131.7, 127.3, 123.4, 121.0, 110.7, 103.7, 37.6, 33.2, 29.8; IR (neat): 2964 (w), 2928 (w), 1953 (w), 1769 (m), 1710 (s), 1615 (w), 1489 (m), 1466 (m), 1430 (m), 1418 (m), 1395 (s), 1362 (m), 1327 (m), 1248 (w), 1207 (w), 1190 (w), $1168(\mathrm{w}), 1111(\mathrm{~s}), 1089(\mathrm{w}), 1073(\mathrm{~m}), 1001(\mathrm{~m}), 954(\mathrm{~m}), 938(\mathrm{w}), 899(\mathrm{w}), 850(\mathrm{w}), 836(\mathrm{~m})$, $806(\mathrm{~s}), 770(\mathrm{~m}), 752(\mathrm{w}), 725(\mathrm{~s}), 708$ (s), $693(\mathrm{~m}), 686(\mathrm{~m}), 611(\mathrm{~m}), 584(\mathrm{~m}), 540(\mathrm{~m}), 529$ (s), $498(\mathrm{w}), 486(\mathrm{~m}) \mathrm{cm}^{-1}$; HRMS (ESI) $\mathrm{m} / z$ : exact mass calculated for $\mathrm{C}_{22} \mathrm{H}_{20} \mathrm{BrNNaO}_{2}[\mathrm{M}+\mathrm{Na}]^{+}$ 432.0570, found 432.0569; SFC (Jasco 2080 Plus, Daicel Chiralcel OJ-H, 95\% CO $2,5 \% \mathrm{MeOH}$ at 100 bar, flow rate $2.0 \mathrm{~mL} \cdot \mathrm{min}^{-1}, 25^{\circ} \mathrm{C}$, detection $218 \mathrm{~nm}$ ): $98 \%$ ee, $\mathrm{t}_{\mathrm{R}}$ (major enantiomer) $8.8 \mathrm{~min}, \mathrm{t}_{\mathrm{R}}$ (minor enantiomer) $9.9 \mathrm{~min}$.

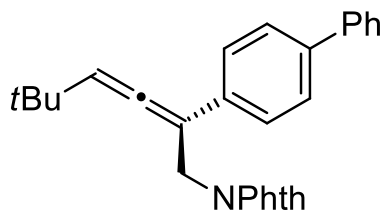

(+)-(R)-2-(2-([1,1'-Biphenyl]-4-yl)-5,5-dimethylhexa-2,3-dien-1-yl)isoindoline-1,3-dione (3g). General procedure $\mathrm{C}$ was followed but deionized water $(90.0 \mu \mathrm{L}, 5.00 \mathrm{mmol}, 10.0$ equiv.) was added subsequent to the addition of $\mathrm{K}_{3} \mathrm{PO}_{4}$. (-)-(S)-6-(1,3-dioxoisoindolin-2-yl)-2,2-dimethylhex-4-yn-3-yl benzoate (1f) (188 mg, $0.500 \mathrm{mmol}, 97 \%$ ee) and 4-biphenylboronic acid (198 mg, $1.00 \mathrm{mmol})$ were used. The crude product was purified by flash column chromatography on silica gel (cyclohexane/EtOAc 12:1 to 8:1 gradient). The title compound was isolated as an orange solid (195 mg, $0.479 \mathrm{mmol}, 96 \%$ ). Crystals suitable for X-ray crystallographic analysis were obtained by slow evaporation from $\mathrm{CHCl}_{3} / n$-hexane at $24{ }^{\circ} \mathrm{C}$.

TLC: $R_{f}=0.32$ (cyclohexane/EtOAc 5:1; UV, CAM); Melting point: $152-154{ }^{\circ} \mathrm{C}$ $\left(\mathrm{CHCl}_{3} / n\right.$-hexane); Specific Rotation: $[\alpha]_{\mathrm{D}}^{22}+99.4$ (c = 1.00, $\left.\mathrm{CHCl}_{3}\right)$; ${ }^{\mathbf{1}} \mathbf{H}-\mathbf{N M R}\left(400 \mathrm{MHz}, \mathrm{CDCl}_{3}\right.$, $298 \mathrm{~K}): \delta 7.90-7.85(\mathrm{~m}, 2 \mathrm{H}), 7.75-7.70(\mathrm{~m}, 2 \mathrm{H}), 7.62-7.57(\mathrm{~m}, 4 \mathrm{H}), 7.51-7.48(\mathrm{~m}, 2 \mathrm{H}), 7.44-$ $7.42(\mathrm{~m}, 2 \mathrm{H}), 7.37-7.33(\mathrm{~m}, 1 \mathrm{H}), 5.56(\mathrm{t}, J=3.7 \mathrm{~Hz}, 1 \mathrm{H}), 4.81(\mathrm{dd}, J=15.4,3.5 \mathrm{~Hz}, 1 \mathrm{H}), 4.70(\mathrm{dd}$, $J=15.4,3.9 \mathrm{~Hz}, 1 \mathrm{H}), 0.87(\mathrm{~s}, 9 \mathrm{H}) ;{ }^{13} \mathrm{C}-\mathrm{NMR}\left(101 \mathrm{MHz}, \mathrm{CDCl}_{3}, 298 \mathrm{~K}\right): \delta 199.4,168.0,140.8,139.9$, 134.1 (two coincident resonances), 132.4, 128.9, 127.4, 127.3, 127.1, 126.1, 123.4, 110.5, 104.2, 37.8, 
33.2, 29.9; IR (neat): 3033 (w), 2959 (w), 2926 (w), $2867(\mathrm{w}), 1952$ (w), 1770 (m), $1712(\mathrm{~s})$, 1614 (w), 1598 (w), 1486 (m), 1466 (m), 1430 (m), 1417 (w), 1393 (s), 1384 (m), 1362 (w), 1328 (m), 1249 (w), 1202 (w), 1189 (w), 1168 (w), 1109 (s), 1089 (w), 1072 (w), 1003 (w), 955 (m), 941 (w), $925(\mathrm{w}), 850(\mathrm{~m}), 838(\mathrm{~m}), 814(\mathrm{w}), 760$ (s), $741(\mathrm{~m}), 709(\mathrm{~s}), 692(\mathrm{~s}), 648(\mathrm{w}), 607(\mathrm{~m}), 583(\mathrm{~m})$, $556(\mathrm{w}), 531$ (s), $516(\mathrm{w}), 480(\mathrm{w}) \mathrm{cm}^{-1}$; HRMS (ESI) $\mathrm{m} / z$ : exact mass calculated for $\mathrm{C}_{28} \mathrm{H}_{26} \mathrm{NO}_{2}$ $[\mathrm{M}+\mathrm{H}]^{+}$408.1958, found 408.1957; SFC (Jasco 2080 Plus, Daicel Chiralcel OJ-H, 90\% CO, $10 \%$ $\mathrm{MeOH}$ at $100 \mathrm{bar}$, flow rate $2.0 \mathrm{~mL} \cdot \mathrm{min}^{-1}, 25^{\circ} \mathrm{C}$, detection $271 \mathrm{~nm}$ ): $92 \%$ ee, $\mathrm{t}_{\mathrm{R}}$ (major enantiomer) $20.5 \mathrm{~min}, \mathrm{t}_{\mathrm{R}}$ (minor enantiomer) $25.1 \mathrm{~min}$.

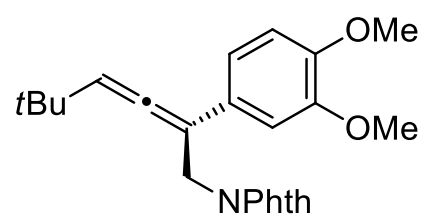

\section{(+)-(R)-2-(2-(3,4-Dimethoxyphenyl)-5,5-dimethylhexa-2,3-dien-1-yl)isoindoline-1,3-dione}

(3h). General procedure $\mathrm{C}$ was followed but deionized water $(90.0 \mu \mathrm{L}, 5.00 \mathrm{mmol}, 10.0$ equiv.) was added subsequent to the addition of $\mathrm{K}_{3} \mathrm{PO}_{4}$. (-)-(S)-6-(1,3-dioxoisoindolin-2-yl)-2,2-dimethylhex-4yn-3-yl benzoate (1f) (188 mg, $0.500 \mathrm{mmol}, 97 \%$ ee ) and 3,4-dimethoxybenzeneboronic acid (182 mg, $1.00 \mathrm{mmol}$ ) were used. The crude product was purified by flash column chromatography on silica gel (cyclohexane/EtOAc 12:1 to 2:1 gradient). The title compound was isolated as a light yellow foam (191 mg, $0.487 \mathrm{mmol}, 97 \%$ ). Crystals suitable for X-ray crystallographic analysis were obtained by slow evaporation from $\mathrm{CH}_{2} \mathrm{Cl}_{2} / n$-hexane at $24{ }^{\circ} \mathrm{C}$.

TLC: $R_{f}=0.29$ (cyclohexane/EtOAc 3:1; UV, CAM); Melting point: $117-118^{\circ} \mathrm{C}$ $\left(\mathrm{CH}_{2} \mathrm{Cl}_{2} / n\right.$-hexane); Specific Rotation: $[\alpha]_{\mathrm{D}}^{21}+85.7$ (c = 1.00, $\left.\mathrm{CHCl}_{3}\right) ;{ }^{1} \mathbf{H}-\mathbf{N M R}\left(400 \mathrm{MHz}, \mathrm{CDCl}_{3}\right.$, $298 \mathrm{~K}): \delta 7.88-7.84(\mathrm{~m}, 2 \mathrm{H}), 7.74-7.68(\mathrm{~m}, 2 \mathrm{H}), 7.00-6.95(\mathrm{~m}, 2 \mathrm{H}), 6.85(\mathrm{~d}, J=8.2 \mathrm{~Hz}, 1 \mathrm{H}), 5.51$ (t, $J=3.6 \mathrm{~Hz}, 1 \mathrm{H}), 4.75(\mathrm{dd}, J=15.4,3.5 \mathrm{~Hz}, 1 \mathrm{H}), 4.64(\mathrm{dd}, J=15.4,3.7 \mathrm{~Hz}, 1 \mathrm{H}), 3.88-3.87$ (m, 6H), 0.86 (s, 9H); ${ }^{13} \mathrm{C}-\mathbf{N M R}\left(101 \mathrm{MHz}, \mathrm{CDCl}_{3}, 298 \mathrm{~K}\right): \delta$ 198.9, 168.0, 149.1, 148.5, 134.1, 132.4, 127.7, 123.4, 117.7, 111.2, 110.3, 109.5, 104.3, 56.1, 55.9, 37.9, 33.1, 29.9; IR (neat): 2953 (m), 2861 (w), 1772 (m), 1713 (s), 1605 (w), 1587 (w), 1514 (m), 1469 (m), 1455 (w), 1433 (w), 1410 (m), $1393(\mathrm{~m}), 1363(\mathrm{w}), 1342(\mathrm{w}), 1323(\mathrm{~m}), 1259(\mathrm{~m}), 1241(\mathrm{~m}), 1205(\mathrm{w}), 1192(\mathrm{w}), 1166(\mathrm{w})$, $1147(\mathrm{~m}), 1110(\mathrm{~m}), 1089(\mathrm{w}), 1045(\mathrm{w}), 1023(\mathrm{~m}), 975(\mathrm{w}), 952(\mathrm{~m}), 857(\mathrm{~m}), 824(\mathrm{w}), 798(\mathrm{~m})$, 
760 (m), 748 (m), 714 (s), 633 (m), 615 (m), 596 (w), 527 (m), 482 (w) cm ${ }^{-1}$; HRMS (ESI) m/z: exact mass calculated for $\mathrm{C}_{24} \mathrm{H}_{26} \mathrm{NO}_{4}[\mathrm{M}+\mathrm{H}]^{+}$392.1856, found 392.1858; SFC (Waters Acquity UPC ${ }^{2}$, Trefoil, $95 \% \mathrm{CO}_{2}, 5 \% \mathrm{MeOH}$, flow rate $2.0 \mathrm{~mL} \cdot \mathrm{min}^{-1}, 40{ }^{\circ} \mathrm{C}$, detection $254 \mathrm{~nm}$ ): $87 \%$ ee, $\mathrm{t}_{\mathrm{R}}$ (minor enantiomer) $8.4 \mathrm{~min}, \mathrm{t}_{\mathrm{R}}$ (major enantiomer) $9.1 \mathrm{~min}$.

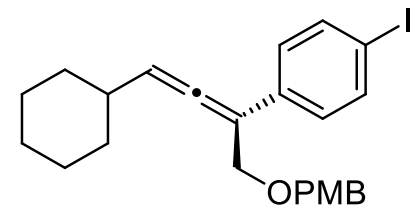

(+)-(R)-1-(4-Cyclohexyl-1-((4-methoxybenzyl)oxy)buta-2,3-dien-2-yl)-4-iodobenzene

(3i).

General procedure $\mathrm{C}$ was followed but $\left[\{\mathrm{Rh}(\mathrm{cod}) \mathrm{OH}\}_{2}\right](6.8 \mathrm{mg}, 0.015 \mathrm{mmol}, 3.0 \mathrm{~mol} \%)$ was used instead of $\left[\{\mathrm{Rh}(\operatorname{cod}) \mathrm{Cl}\}_{2}\right]$ and deionized water $(90.0 \mu \mathrm{L}, 5.00 \mathrm{mmol}, 10.0$ equiv.) was added subsequent to the addition of $\mathrm{K}_{3} \mathrm{PO}_{4}$. (-)-(S)-1-cyclohexyl-4-((4-methoxybenzyl)oxy)but-2-yn-1-yl benzoate (1i) (196 mg, $0.500 \mathrm{mmol}, 98 \%$ ee ) and 4-iodophenylboronic acid (248 mg, $1.00 \mathrm{mmol}$ ) were used. The crude product was purified by flash column chromatography on silica gel (cyclohexane/EtOAc 30:1 to 20:1 gradient). The title compound was isolated as a yellow, clear oil (134 mg, $0.282 \mathrm{mmol}, 57 \%$ ).

TLC: $R_{f}=0.22$ (cyclohexane/EtOAc 20:1; UV, CAM); Specific Rotation: $[\alpha]_{\mathrm{D}}^{22}+99.6(\mathrm{c}=1.00$, $\left.\mathrm{CHCl}_{3}\right) ;{ }^{1} \mathbf{H}-\mathbf{N M R}\left(400 \mathrm{MHz}, \mathrm{CDCl}_{3}, 298 \mathrm{~K}\right): \delta 7.64-7.60(\mathrm{~m}, 2 \mathrm{H}), 7.25-7.21(\mathrm{~m}, 4 \mathrm{H}), 6.89-6.85$ $(\mathrm{m}, 2 \mathrm{H}), 5.57-5.56(\mathrm{~m}, 1 \mathrm{H}), 4.47(\mathrm{~s}, 2 \mathrm{H}), 4.41(\mathrm{~d}, J=1.2 \mathrm{~Hz}, 2 \mathrm{H}), 3.81(\mathrm{~s}, 3 \mathrm{H}), 2.19-2.10(\mathrm{~m}, 1 \mathrm{H})$, $1.85-1.81(\mathrm{~m}, 2 \mathrm{H}), 1.75-1.71(\mathrm{~m}, 2 \mathrm{H}), 1.66-1.62(\mathrm{~m}, 1 \mathrm{H}), 1.36-1.11(\mathrm{~m}, 5 \mathrm{H}) ;{ }^{13} \mathbf{C}-\mathbf{N M R}$ (101 MHz, $\left.\mathrm{CDCl}_{3}, 298 \mathrm{~K}\right): \delta 204.4,159.4,137.5,135.3,130.3,129.7,128.3,113.9,102.9,100.7,92.0$, 71.2, 69.7, 55.4, 37.9, 33.4, 33.3, 26.2, 26.14, 26.13; IR (neat): $2999(\mathrm{w}), 2922(\mathrm{~m}), 2849$ (m), 1945 (w), 1612 (m), 1585 (w), 1512 (s), 1484 (m), 1463 (w), 1447 (m), 1381 (w), 1348 (w), 1302 (w), 1246 (s), 1172 (m), 1076 (s), 1063 (s), 1036 (s), 1003 (s), 949 (w), 910 (w), 890 (w), 823 (s), 807 (s), 757 (m), 738 (m), 709 (w), 637 (w), 629 (w), 580 (m), $518(\mathrm{~m}) \mathrm{cm}^{-1}$; HRMS (ESI) m/z: exact mass calculated for $\mathrm{C}_{24} \mathrm{H}_{31} \mathrm{INO}_{2}\left[\mathrm{M}+\mathrm{NH}_{4}\right]^{+}$492.1394, found 492.1392; SFC (Jasco 2080 Plus, Daicel Chiralcel OJ-H, $80 \% \mathrm{CO}_{2}, 20 \% \mathrm{MeOH}$ at 100 bar, flow rate $2.0 \mathrm{~mL} \cdot \mathrm{min}^{-1}, 25^{\circ} \mathrm{C}$, detection $254 \mathrm{~nm}$ ): $91 \% e e, t_{R}$ (major enantiomer) $23.4 \mathrm{~min}, \mathrm{t}_{\mathrm{R}}$ (minor enantiomer) $30.1 \mathrm{~min}$. 


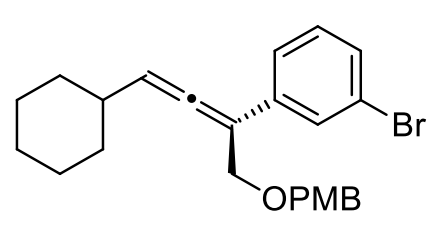

(+)-(R)-1-Bromo-3-(4-cyclohexyl-1-((4-methoxybenzyl)oxy)buta-2,3-dien-2-yl)benzene (3j). General procedure $\mathrm{C}$ was followed deionized water $(90.0 \mu \mathrm{L}, 5.00 \mathrm{mmol}, 10.0$ equiv.) was added subsequent to the addition of $\mathrm{K}_{3} \mathrm{PO}_{4}$. (-)-(S)-1-cyclohexyl-4-((4-methoxybenzyl)oxy)but-2-yn-1-yl benzoate (1i) (196 mg, $0.500 \mathrm{mmol}, 98 \%$ ee) and 3-bromophenylboronic acid (201 mg, $1.00 \mathrm{mmol}$ ) were used. The crude product was purified by flash column chromatography on silica gel (cyclohexane/EtOAc 30:1 to 20:1 gradient). The title compound was isolated as a yellow, clear oil (134 mg, $0.314 \mathrm{mmol}, 63 \%)$.

TLC: $R_{f}=0.34$ (cyclohexane/EtOAc 12:1; UV, CAM); Specific Rotation: $[\alpha]_{\mathrm{D}}^{22}+88.8(\mathrm{c}=1.00$, $\left.\mathrm{CHCl}_{3}\right) ;{ }^{1} \mathbf{H}-\mathbf{N M R}\left(400 \mathrm{MHz}, \mathrm{CDCl}_{3}, 298 \mathrm{~K}\right): \delta 7.62(\mathrm{t}, J=1.8 \mathrm{~Hz}, 1 \mathrm{H}), 7.43-7.62(\mathrm{~m}, 1 \mathrm{H}), 7.34-$ $7.31(\mathrm{~m}, 1 \mathrm{H}), 7.27-7.24(\mathrm{~m}, 2 \mathrm{H}), 7.17(\mathrm{t}, J=7.9 \mathrm{~Hz}, 1 \mathrm{H}), 6.90-6.86(\mathrm{~m}, 2 \mathrm{H}), 5.61-5.60(\mathrm{~m}, 1 \mathrm{H})$, $4.49(\mathrm{~s}, 2 \mathrm{H}), 4.40(\mathrm{~d}, J=1.2 \mathrm{~Hz}, 2 \mathrm{H}), 3.81(\mathrm{~s}, 3 \mathrm{H}), 2.21-2.12(\mathrm{~m}, 1 \mathrm{H}), 1.87-1.82(\mathrm{~m}, 2 \mathrm{H}), 1.77-$ $1.72(\mathrm{~m}, 2 \mathrm{H}), 1.67-1.63(\mathrm{~m}, 1 \mathrm{H}), 1.37-1.13(\mathrm{~m}, 5 \mathrm{H}) . ;{ }^{13} \mathbf{C}-\mathbf{N M R}\left(101 \mathrm{MHz}, \mathrm{CDCl}_{3}, 298 \mathrm{~K}\right): \delta 204.6$, $159.4,138.0,130.3,129.9,129.7,129.7,129.3,124.9,122.7,113.9,102.6,100.8,71.3,69.6,55.4$, 37.9, 33.4, 33.3, 26.2, 26.1 (two coincident resonances); IR (neat): 2999 (w), 2911 (s), 2849 (m), 1945 (w), 1612 (m), 1589 (m), 1559 (m), 1512 (s), 1474 (m), 1464 (m), 1448 (m), 1381 (w), 1348 (w), $1302(\mathrm{~m}), 1246(\mathrm{~s}), 1172(\mathrm{~m}), 1070(\mathrm{~s}), 1036(\mathrm{~s}), 995(\mathrm{w}), 948(\mathrm{w}), 890(\mathrm{w}), 819(\mathrm{~m}), 784(\mathrm{~m})$, 756 (w), 733 (w), 690 (m), 665 (w), 575 (w), 517 (w) cm c $^{-1}$ HRMS (ESI) m/z: exact mass calculated for $\mathrm{C}_{24} \mathrm{H}_{31} \mathrm{BrNO}_{2}\left[\mathrm{M}+\mathrm{NH}_{4}\right]^{+}$444.1533, found 444.1534; SFC (Jasco 2080 Plus, Daicel Chiralpak AS-H, $99 \% \mathrm{CO}_{2}, 1 \% \mathrm{MeOH}$ at $100 \mathrm{bar}$, flow rate $2.0 \mathrm{~mL} \cdot \mathrm{min}^{-1}, 25^{\circ} \mathrm{C}$, detection $254 \mathrm{~nm}$ ): $90 \%$ ee, $\mathrm{t}_{\mathrm{R}}$ (minor enantiomer) $29.7 \mathrm{~min}, \mathrm{t}_{\mathrm{R}}$ (major enantiomer) $31.3 \mathrm{~min}$.

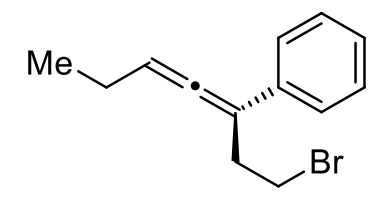

(+)-(S)-(1-Bromohepta-3,4-dien-3-yl)benzene (3k). General procedure C was followed but the reaction was conducted on a $0.200 \mathrm{mmol}$ scale and stirred at $40{ }^{\circ} \mathrm{C}$ for $12 \mathrm{~h}$. (-)-(S)-7-bromohept-4- 
yn-3-yl benzoate (1k) $(59.0 \mathrm{mg}, 0.200 \mathrm{mmol}, 86 \%$ ee) and phenylboronic acid (49.0 mg, $0.400 \mathrm{mmol})$ were used. The crude product was purified by flash column chromatography on silica gel (cyclohexane/EtOAc 80:1). The title compound was isolated as a pale brown, clear oil (31.0 $\mathrm{mg}$, $0.123 \mathrm{mmol}, 62 \%)$.

TLC: $R_{f}=0.42$ (cyclohexane/EtOAc 30:1; UV, CAM); Specific Rotation: $[\alpha]_{\mathrm{D}}^{22}+32.0(\mathrm{c}=1.00$, $\mathrm{CHCl}_{3}$ ); ${ }^{1} \mathrm{H}-\mathrm{NMR}\left(400 \mathrm{MHz}, \mathrm{CDCl}_{3}, 298 \mathrm{~K}\right): \delta 7.40-7.31$ (m, 4H), $7.24-7.20$ (m, 1H), 5.67 (tt, $J=6.1,3.0 \mathrm{~Hz}, 1 \mathrm{H}), 3.55(\mathrm{t}, J=7.6 \mathrm{~Hz}, 2 \mathrm{H}), 3.04-2.95(\mathrm{~m}, 2 \mathrm{H}), 2.21-2.14(\mathrm{~m}, 2 \mathrm{H}), 1.10(\mathrm{t}$, $J=7.4 \mathrm{~Hz}, 3 \mathrm{H}) . ;{ }^{13} \mathbf{C}-\mathbf{N M R}\left(101 \mathrm{MHz}, \mathrm{CDCl}_{3}, 298 \mathrm{~K}\right): \delta 203.4,136.5,128.6,126.9,125.8,104.4$, 97.9, 33.7, 30.8, 22.3, 13.7; IR (film, $\mathrm{CHCl}_{3}$ ): 3060 (w), 3028 (w), 2964 (m), 2930 (w), 2872 (w), $1946(w), 1597(w), 1494(m), 1451(\mathrm{~m}), 1374(\mathrm{w}), 1323(\mathrm{w}), 1269(\mathrm{w}), 1204(\mathrm{~m}), 1069(\mathrm{w})$, 1031 (w), $812(\mathrm{w}), 754(\mathrm{~m}), 730$ (w), $692(\mathrm{~s}), 597(\mathrm{~m}) \mathrm{cm}^{-1}$; HRMS (EI) m/z: exact mass calculated for $\mathrm{C}_{13} \mathrm{H}_{14} \mathrm{Br}[\mathrm{M}]^{+}$250.0352, found 250.0351; SFC (Jasco 2080 Plus, Daicel Chiralcel OB-H, 100\% $\mathrm{CO}_{2}$ at $100 \mathrm{bar}$, flow rate $2.0 \mathrm{~mL} \cdot \mathrm{min}^{-1}, 25^{\circ} \mathrm{C}$, detection $221 \mathrm{~nm}$ ): $83 \% e e, \mathrm{t}_{\mathrm{R}}$ (major enantiomer) $12.6 \mathrm{~min}, \mathrm{t}_{\mathrm{R}}$ (minor enantiomer) $13.4 \mathrm{~min}$. 


\section{X-Ray Crystallographic Data}

\section{X-Ray Crystallographic Data for (+)-3f}

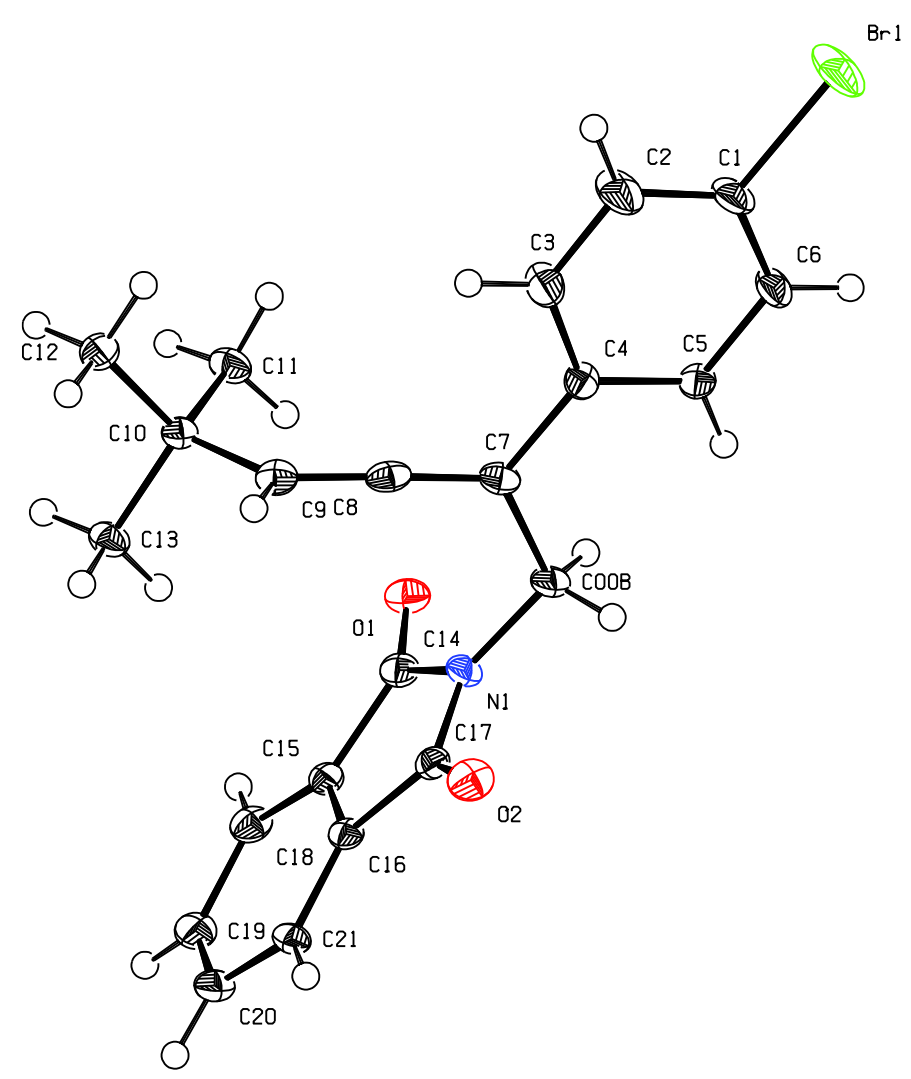

ORTEP view ${ }^{8}$ of (+)-3f, the thermal ellipsoids are drawn at the $50 \%$ probability level.

Database Reference. Crystallographic data have been deposited with the Cambridge Crystallographic Data Centre (CCDC) as supplementary publication no. CCDC-1437268. Data can be obtained free of charge on application to CCDC.

Experimental. A suitable clear, colorless block was selected, mounted in perfluoroalkyl polyether oil on polyimide Micromounts (supplied by MiTeGen) and measured on a Bruker/Nonius Kappa Apex II diffractometer with a Bruker Apex II area detector. The detector type was a CCD area detector. The crystal was kept at 100.0(2) K during data collection. Using Olex $2,{ }^{9}$ the structure was solved with the XS structure solution program ${ }^{10}$ using Direct Methods and refined with the XL refinement package ${ }^{10}$

\footnotetext{
${ }^{8}$ All ORTEP views were generated using the Platon software (version 70414) by A. L. Spek, see: Spek, A. L. Acta Crystallogr., Sect. D: Biol. Crystallogr. 2009, 65, 148.

${ }^{9}$ Dolomanov, O. V.; Bourhis, L. J.; Gildea, R. J.; Howard, J. A. K.; Puschmann, H. J. Appl. Crystallogr. 2009, 42, 339.

${ }^{10}$ Sheldrick, G. M. Acta Crystallogr., Sect. A: Found. Crystallogr. 2008, 64, 112. 
using Least Squares minimization. The absolute stereochemistry was not determined by X-Ray diffraction.

Table A1. Crystal data and structure refinement.

\begin{tabular}{|c|c|}
\hline Empirical formula & $\mathrm{C}_{22} \mathrm{H}_{20} \mathrm{BrNO}_{2}$ \\
\hline Formula weight & 410.30 \\
\hline Temperature/K & $100.0(2)$ \\
\hline Crystal system & monoclinic \\
\hline Space group & $\mathrm{P} 2{ }_{1}$ \\
\hline $\mathrm{a} / \AA$ & $8.6651(6)$ \\
\hline $\mathrm{b} / \AA$ & $5.8524(3)$ \\
\hline $\mathrm{c} / \AA$ & $18.9495(10)$ \\
\hline$\alpha /^{\circ}$ & 90 \\
\hline$\beta /{ }^{\circ}$ & $93.712(5)$ \\
\hline$\gamma /{ }^{\circ}$ & 90 \\
\hline Volume $/ \AA^{3}$ & $958.94(10)$ \\
\hline $\mathrm{Z}$ & 2 \\
\hline$\rho_{\text {calc }} \mathrm{g} / \mathrm{cm}^{3}$ & 1.421 \\
\hline$\mu / \mathrm{mm}^{-1}$ & 2.159 \\
\hline $\mathrm{F}(000)$ & 420.0 \\
\hline Crystal size $/ \mathrm{mm}^{3}$ & $0.28 \times 0.11 \times 0.07$ \\
\hline Radiation & $\operatorname{MoK} \alpha(\lambda=0.71073)$ \\
\hline $2 \Theta$ range for data collection $/^{\circ}$ & 4.71 to 61.33 \\
\hline Index ranges & $-12 \leq \mathrm{h} \leq 12,-8 \leq \mathrm{k} \leq 8,-26 \leq 1 \leq 25$ \\
\hline Reflections collected & 5272 \\
\hline Independent reflections & $5272\left[\mathrm{R}_{\mathrm{int}}=0.0688, \mathrm{R}_{\text {sigma }}=0.0274\right]$ \\
\hline Data/restraints/parameters & $5272 / 73 / 239$ \\
\hline Goodness-of-fit on $\mathrm{F}^{2}$ & 1.091 \\
\hline Final $\mathrm{R}$ indexes $[\mathrm{I}>=2 \sigma(\mathrm{I})]$ & $\mathrm{R}_{1}=0.0589, \mathrm{wR}_{2}=0.1301$ \\
\hline Final $\mathrm{R}$ indexes [all data] & $\mathrm{R}_{1}=0.0720, \mathrm{wR}_{2}=0.1410$ \\
\hline Largest diff. peak/hole / e $\AA^{-3}$ & $0.47 /-0.66$ \\
\hline Flack parameter & $0.047(15)$ \\
\hline
\end{tabular}

Table A2. Fractional Atomic Coordinates $\left(\times 10^{4}\right)$ and Equivalent Isotropic Displacement Parameters $\left(\AA^{2} \times 10^{3}\right)$. $\mathrm{U}_{\text {eq }}$ is defined as $1 / 3$ of the trace of the orthogonalised $\mathrm{U}_{\mathrm{IJ}}$ tensor.

$\begin{array}{lllll}\text { Atom } & \boldsymbol{x} & \boldsymbol{y} & \boldsymbol{z} & \mathbf{U}(\mathbf{e q}) \\ \text { Br1 } & 1991.3(12) & -927.0(15) & 5502.3(4) & 43.3(3)\end{array}$




$\begin{array}{lllll}\text { O1 } & 3650(5) & 3681(8) & 1127(2) & 21.4(11) \\ \text { O2 } & 106(5) & 8881(11) & 1816(2) & 23.8(10) \\ \text { N1 } & 1751(6) & 5888(9) & 1596(3) & 17.4(11) \\ \text { C00B } & 1498(7) & 4479(11) & 2208(3) & 20.9(15) \\ \text { C1 } & 2163(9) & 941(12) & 4687(4) & 24.8(14) \\ \text { C2 } & 3115(10) & 2832(14) & 4729(4) & 33.2(18) \\ \text { C3 } & 3211(8) & 4191(17) & 4134(3) & 28.2(15) \\ \text { C4 } & 2379(7) & 3669(11) & 3497(3) & 17.1(12) \\ \text { C5 } & 1435(8) & 1726(12) & 3475(3) & 18.0(12) \\ \text { C6 } & 1331(8) & 339(12) & 4075(3) & 22.0(14) \\ \text { C7 } & 2519(8) & 5114(11) & 2859(3) & 17.9(12) \\ \text { C8 } & 3482(8) & 6842(12) & 2846(3) & 18.7(13) \\ \text { C9 } & 4460(7) & 8512(10) & 2793(3) & 18.9(13) \\ \text { C10 } & 6083(7) & 8323(11) & 2546(3) & 17.5(12) \\ \text { C11 } & 6566(8) & 5828(11) & 2484(4) & 20.7(13) \\ \text { C12 } & 7201(8) & 9546(11) & 3079(4) & 22.5(15) \\ \text { C13 } & 6111(8) & 9492(11) & 1820(3) & 19.4(14) \\ \text { C14 } & 2821(8) & 5350(11) & 1101(3) & 18.5(13) \\ \text { C15 } & 2658(7) & 7178(11) & 552(3) & 16.5(12) \\ \text { C16 } & 1584(6) & 8732(11) & 762(3) & 15.1(12) \\ \text { C17 } & 1020(7) & 7964(11) & 1450(3) & 16.1(12) \\ \text { C18 } & 3377(8) & 7401(12) & -77(3) & 20.3(13) \\ \text { C19 } & 2954(8) & 9314(18) & -493(3) & 23.8(16) \\ \text { C20 } & 1888(8) & 10892(12) & -279(3) & 22.3(13) \\ \text { C21 } & 1161(8) & 10622(11) & 354(3) & 17.5(13)\end{array}$

Table A3. Anisotropic Displacement Parameters $\left(\AA^{2} \times 10^{3}\right)$. The Anisotropic displacement factor exponent takes the form: $-2 \pi^{2}\left[h^{2} a^{* 2} U_{11}+2 h k a * b * U_{12}+\ldots\right]$.

$\begin{array}{lllllll}\text { Atom } & \mathbf{U}_{11} & \mathbf{U}_{22} & \mathbf{U}_{33} & \mathbf{U}_{23} & \mathbf{U}_{13} & \mathbf{U}_{12} \\ \text { Br1 } & 77.0(6) & 37.1(4) & 14.9(3) & 10.3(4) & -5.7(3) & -21.7(5) \\ \text { O1 } & 24(2) & 22(3) & 18(2) & -1.1(19) & -3.5(17) & 3.1(19) \\ \text { O2 } & 25(2) & 31(3) & 15.6(19) & -4(2) & 2.5(16) & 2(2) \\ \text { N1 } & 21(3) & 19(3) & 12(2) & 4(2) & -1(2) & -2(2) \\ \text { C00B } & 24(3) & 24(4) & 14(3) & 5(2) & -5(2) & -3(3) \\ \text { C1 } & 35(4) & 25(3) & 14(3) & 7(2) & -2(3) & -4(3) \\ \text { C2 } & 51(5) & 34(4) & 14(3) & 5(3) & -5(3) & -13(3) \\ \text { C3 } & 38(4) & 28(3) & 18(3) & 2(3) & -5(2) & -15(3) \\ \text { C4 } & 17(3) & 20(3) & 14(2) & -1(2) & 2.9(19) & -1(2)\end{array}$




$\begin{array}{lllllll}\text { C5 } & 20(3) & 24(3) & 10(3) & -1(2) & -1(2) & -4(2) \\ \text { C6 } & 29(3) & 24(3) & 14(3) & 5(2) & 2(2) & -7(3) \\ \text { C7 } & 22(3) & 21(3) & 11(3) & 1(2) & 0(2) & 2(2) \\ \text { C8 } & 19(3) & 27(3) & 10(3) & -2(2) & -1(2) & 6(3) \\ \text { C9 } & 22(3) & 19(3) & 15(3) & -3(2) & -1(2) & 2(2) \\ \text { C10 } & 18(3) & 19(3) & 15(3) & 2(2) & -2(2) & -1(2) \\ \text { C11 } & 26(3) & 14(3) & 21(3) & 2(2) & -1(3) & 3(2) \\ \text { C12 } & 25(3) & 23(4) & 18(3) & 0(2) & -6(2) & -6(3) \\ \text { C13 } & 27(3) & 18(4) & 14(3) & 1(2) & -2(2) & -1(2) \\ \text { C14 } & 20(3) & 22(3) & 13(3) & -1(2) & -2(2) & -1(3) \\ \text { C15 } & 18(3) & 16(3) & 14(3) & -1(2) & -4(2) & -3(2) \\ \text { C16 } & 16(3) & 19(3) & 10(2) & -1(2) & -1(2) & -1(2) \\ \text { C17 } & 14(3) & 19(3) & 15(3) & 0(2) & -1(2) & -1(2) \\ \text { C18 } & 19(3) & 28(3) & 14(3) & -2(3) & 0(2) & 1(3) \\ \text { C19 } & 22(3) & 38(5) & 12(3) & 5(3) & 0.9(19) & -2(3) \\ \text { C20 } & 24(3) & 24(3) & 18(3) & 5(2) & -6(3) & -2(3) \\ \text { C21 } & 21(3) & 18(3) & 12(3) & 2(2) & -1(2) & 1(2)\end{array}$

Table A4. Bond Lengths.

\begin{tabular}{|c|c|c|c|c|c|}
\hline Atom & Atom & Length/Å & Atom & Atom & Length/Å \\
\hline Br1 & $\mathrm{C} 1$ & $1.907(7)$ & $\mathrm{C} 7$ & $\mathrm{C} 8$ & $1.312(10)$ \\
\hline $\mathrm{O} 1$ & $\mathrm{C} 14$ & $1.212(8)$ & $\mathrm{C} 8$ & C9 & $1.302(9)$ \\
\hline $\mathrm{O} 2$ & C17 & $1.211(8)$ & C9 & $\mathrm{C} 10$ & $1.515(9)$ \\
\hline N1 & $\mathrm{C} 00 \mathrm{~B}$ & $1.451(8)$ & $\mathrm{C} 10$ & C11 & $1.525(9)$ \\
\hline N1 & C14 & $1.396(9)$ & $\mathrm{C} 10$ & $\mathrm{C} 12$ & $1.531(9)$ \\
\hline N1 & $\mathrm{C} 17$ & $1.390(8)$ & $\mathrm{C} 10$ & C13 & $1.538(9)$ \\
\hline C00B & $\mathrm{C} 7$ & $1.518(9)$ & C14 & $\mathrm{C} 15$ & $1.493(9)$ \\
\hline $\mathrm{C} 1$ & $\mathrm{C} 2$ & $1.379(10)$ & $\mathrm{C} 15$ & $\mathrm{C} 16$ & $1.378(9)$ \\
\hline $\mathrm{C} 1$ & C6 & $1.371(10)$ & C15 & C18 & $1.386(9)$ \\
\hline $\mathrm{C} 2$ & $\mathrm{C} 3$ & $1.387(10)$ & $\mathrm{C} 16$ & $\mathrm{C} 17$ & $1.492(9)$ \\
\hline $\mathrm{C} 3$ & $\mathrm{C} 4$ & $1.398(8)$ & $\mathrm{C} 16$ & $\mathrm{C} 21$ & $1.385(9)$ \\
\hline $\mathrm{C} 4$ & $\mathrm{C} 5$ & $1.400(9)$ & C18 & C19 & $1.404(11)$ \\
\hline $\mathrm{C} 4$ & $\mathrm{C} 7$ & $1.487(9)$ & C19 & $\mathrm{C} 20$ & $1.386(11)$ \\
\hline $\mathrm{C} 5$ & C6 & $1.403(9)$ & C20 & $\mathrm{C} 21$ & $1.400(9)$ \\
\hline
\end{tabular}

Table A5. Bond Angles.

Atom Atom Atom Angle/ ${ }^{\circ}$ Atom Atom Atom Angle ${ }^{\circ}$ $\begin{array}{llll}\text { C14 N1 C00B 123.4(6) } & \text { N1 }\end{array}$

C9 $\quad \mathrm{C} 10 \quad \mathrm{C} 13 \quad 108.2(5)$ 


$\begin{array}{llll}\mathrm{C} 17 & \mathrm{~N} 1 & \mathrm{C} 00 \mathrm{~B} & 124.4(6) \\ \mathrm{C} 17 & \mathrm{~N} 1 & \mathrm{C} 14 & 112.1(5) \\ \mathrm{N} 1 & \mathrm{C} 00 \mathrm{~B} & \mathrm{C} 7 & 113.6(5) \\ \mathrm{C} 2 & \mathrm{C} 1 & \mathrm{~B} 1 & 119.3(5) \\ \mathrm{C} 6 & \mathrm{C} 1 & \mathrm{~B} 1 & 118.3(5) \\ \mathrm{C} 6 & \mathrm{C} 1 & \mathrm{C} 2 & 122.4(6) \\ \mathrm{C} 1 & \mathrm{C} 2 & \mathrm{C} 3 & 118.6(7) \\ \mathrm{C} 2 & \mathrm{C} 3 & \mathrm{C} 4 & 121.5(7) \\ \mathrm{C} 3 & \mathrm{C} 4 & \mathrm{C} 5 & 118.1(6) \\ \mathrm{C} 3 & \mathrm{C} 4 & \mathrm{C} 7 & 120.8(6) \\ \mathrm{C} 5 & \mathrm{C} 4 & \mathrm{C} 7 & 121.1(6) \\ \mathrm{C} 4 & \mathrm{C} 5 & \mathrm{C} 6 & 120.9(6) \\ \mathrm{C} 1 & \mathrm{C} 6 & \mathrm{C} 5 & 118.6(6) \\ \mathrm{C} 4 & \mathrm{C} 7 & \mathrm{C} 00 \mathrm{~B} & 116.6(5) \\ \mathrm{C} 8 & \mathrm{C} 7 & \mathrm{C} 00 \mathrm{~B} & 120.7(6) \\ \mathrm{C} 8 & \mathrm{C} 7 & \mathrm{C} 4 & 122.6(6) \\ \mathrm{C} 9 & \mathrm{C} 8 & \mathrm{C} 7 & 176.3(7) \\ \mathrm{C} 8 & \mathrm{C} 9 & \mathrm{C} 10 & 126.2(6) \\ \mathrm{C} 9 & \mathrm{C} 10 & \mathrm{C} 11 & 111.0(5) \\ \mathrm{C} 9 & \mathrm{C} 10 & \mathrm{C} 12 & 108.8(5)\end{array}$

$\begin{array}{llll}\mathrm{C} 11 & \mathrm{C} 10 & \mathrm{C} 12 & 109.5(5) \\ \mathrm{C} 11 & \mathrm{C} 10 & \mathrm{C} 13 & 109.7(5) \\ \mathrm{C} 12 & \mathrm{C} 10 & \mathrm{C} 13 & 109.6(5) \\ \mathrm{O} 1 & \mathrm{C} 14 & \mathrm{~N} 1 & 124.9(6) \\ \mathrm{O} 1 & \mathrm{C} 14 & \mathrm{C} 15 & 129.4(6) \\ \mathrm{N} 1 & \mathrm{C} 14 & \mathrm{C} 15 & 105.7(6) \\ \mathrm{C} 16 & \mathrm{C} 15 & \mathrm{C} 14 & 107.8(6) \\ \mathrm{C} 16 & \mathrm{C} 15 & \mathrm{C} 18 & 122.4(6) \\ \mathrm{C} 18 & \mathrm{C} 15 & \mathrm{C} 14 & 129.8(6) \\ \mathrm{C} 15 & \mathrm{C} 16 & \mathrm{C} 17 & 108.7(6) \\ \mathrm{C} 15 & \mathrm{C} 16 & \mathrm{C} 21 & 121.7(6) \\ \mathrm{C} 21 & \mathrm{C} 16 & \mathrm{C} 17 & 129.7(6) \\ \mathrm{O} 2 & \mathrm{C} 17 & \mathrm{~N} 1 & 125.4(6) \\ \mathrm{O} 2 & \mathrm{C} 17 & \mathrm{C} 16 & 129.1(6) \\ \mathrm{N} 1 & \mathrm{C} 17 & \mathrm{C} 16 & 105.5(5) \\ \mathrm{C} 15 & \mathrm{C} 18 & \mathrm{C} 19 & 116.3(6) \\ \mathrm{C} 20 & \mathrm{C} 19 & \mathrm{C} 18 & 121.5(6) \\ \mathrm{C} 19 & \mathrm{C} 20 & \mathrm{C} 21 & 121.4(6) \\ \mathrm{C} 16 & \mathrm{C} 21 & \mathrm{C} 20 & 116.8(6)\end{array}$

Table A6. Torsion Angles.

\begin{tabular}{|c|c|c|c|c|c|c|c|c|c|}
\hline $\mathbf{A}$ & B & C & D & Angle ${ }^{\circ}$ & $\mathbf{A}$ & B & C & D & Angle ${ }^{\circ}$ \\
\hline $\mathrm{Br} 1$ & $\mathrm{C} 1$ & $\mathrm{C} 2$ & $\mathrm{C} 3$ & $-179.1(7)$ & $\mathrm{C} 8$ & C9 & $\mathrm{C} 10$ & C11 & $-10.0(9)$ \\
\hline $\mathrm{Br} 1$ & $\mathrm{C} 1$ & C6 & $\mathrm{C} 5$ & 179.1(5) & C8 & C9 & $\mathrm{C} 10$ & $\mathrm{C} 12$ & $-130.5(7)$ \\
\hline $\mathrm{O} 1$ & C14 & $\mathrm{C} 15$ & C16 & $-179.1(6)$ & $\mathrm{C} 8$ & C9 & $\mathrm{C} 10$ & $\mathrm{C} 13$ & $110.4(7)$ \\
\hline $\mathrm{O} 1$ & $\mathrm{C} 14$ & C15 & $\mathrm{C} 18$ & $2.1(12)$ & C14 & N1 & $\mathrm{COOB}$ & C7 & $90.4(7)$ \\
\hline N1 & $\mathrm{C} 00 \mathrm{~B}$ & $\mathrm{C} 7$ & $\mathrm{C} 4$ & $-177.1(5)$ & C14 & N1 & $\mathrm{C} 17$ & $\mathrm{O} 2$ & $-175.9(6)$ \\
\hline N1 & $\mathrm{COOB}$ & C7 & $\mathrm{C} 8$ & $2.1(9)$ & C14 & N1 & $\mathrm{C} 17$ & C16 & $5.0(7)$ \\
\hline N1 & C14 & C15 & C16 & $2.9(7)$ & C14 & C15 & C16 & $\mathrm{C} 17$ & $0.0(7)$ \\
\hline $\mathrm{N} 1$ & C14 & $\mathrm{C} 15$ & $\mathrm{C} 18$ & $-175.8(6)$ & C14 & C15 & $\mathrm{C} 16$ & $\mathrm{C} 21$ & $-179.0(6)$ \\
\hline $\mathrm{COOB}$ & N1 & $\mathrm{C} 14$ & $\mathrm{O} 1$ & $-0.4(10)$ & $\mathrm{C} 14$ & $\mathrm{C} 15$ & $\mathrm{C} 18$ & C19 & $178.8(7)$ \\
\hline $\mathrm{C} 00 \mathrm{~B}$ & N1 & $\mathrm{C} 14$ & $\mathrm{C} 15$ & $177.6(5)$ & $\mathrm{C} 15$ & C16 & $\mathrm{C} 17$ & $\mathrm{O} 2$ & $178.0(7)$ \\
\hline $\mathrm{C} 00 \mathrm{~B}$ & N1 & $\mathrm{C} 17$ & $\mathrm{O} 2$ & $1.4(10)$ & $\mathrm{C} 15$ & C16 & $\mathrm{C} 17$ & N1 & $-2.9(7)$ \\
\hline $\mathrm{C} 00 \mathrm{~B}$ & N1 & $\mathrm{C} 17$ & C16 & $-177.7(5)$ & $\mathrm{C} 15$ & C16 & $\mathrm{C} 21$ & $\mathrm{C} 20$ & $-0.6(9)$ \\
\hline $\mathrm{C} 1$ & $\mathrm{C} 2$ & $\mathrm{C} 3$ & $\mathrm{C} 4$ & $-0.6(13)$ & $\mathrm{C} 15$ & C18 & C19 & $\mathrm{C} 20$ & $0.6(11)$ \\
\hline $\mathrm{C} 2$ & $\mathrm{C} 1$ & C6 & $\mathrm{C} 5$ & $-1.2(12)$ & C16 & C15 & $\mathrm{C} 18$ & C19 & $0.2(10)$ \\
\hline $\mathrm{C} 2$ & $\mathrm{C} 3$ & $\mathrm{C} 4$ & C5 & $0.2(12)$ & $\mathrm{C} 17$ & N1 & $\mathrm{C} 00 \mathrm{~B}$ & $\mathrm{C} 7$ & $-86.7(7)$ \\
\hline $\mathrm{C} 2$ & $\mathrm{C} 3$ & $\mathrm{C} 4$ & $\mathrm{C} 7$ & $-178.7(8)$ & $\mathrm{C} 17$ & N1 & C14 & $\mathrm{O} 1$ & $176.9(6)$ \\
\hline
\end{tabular}




$\begin{array}{llllllllll}\mathrm{C} 3 & \mathrm{C} 4 & \mathrm{C} 5 & \mathrm{C} 6 & -0.2(10) & \mathrm{C} 17 & \mathrm{~N} 1 & \mathrm{C} 14 & \mathrm{C} 15 & -5.0(7) \\ \mathrm{C} 3 & \mathrm{C} 4 & \mathrm{C} 7 & \mathrm{C} 00 \mathrm{~B} & -176.6(6) & \mathrm{C} 17 & \mathrm{C} 16 & \mathrm{C} 21 & \mathrm{C} 20 & -179.4(6) \\ \mathrm{C} 3 & \mathrm{C} 4 & \mathrm{C} 7 & \mathrm{C} 8 & 4.2(10) & \mathrm{C} 18 & \mathrm{C} 15 & \mathrm{C} 16 & \mathrm{C} 17 & 178.9(6) \\ \mathrm{C} 4 & \mathrm{C} 5 & \mathrm{C} 6 & \mathrm{C} 1 & 0.7(11) & \mathrm{C} 18 & \mathrm{C} 15 & \mathrm{C} 16 & \mathrm{C} 21 & -0.2(10) \\ \mathrm{C} 5 & \mathrm{C} 4 & \mathrm{C} 7 & \mathrm{C} 00 \mathrm{~B} & 4.6(9) & \mathrm{C} 18 & \mathrm{C} 19 & \mathrm{C} 20 & \mathrm{C} 21 & -1.3(11) \\ \mathrm{C} 5 & \mathrm{C} 4 & \mathrm{C} 7 & \mathrm{C} 8 & -174.6(6) & \mathrm{C} 19 & \mathrm{C} 20 & \mathrm{C} 21 & \mathrm{C} 16 & 1.3(10) \\ \mathrm{C} 6 & \mathrm{C} 1 & \mathrm{C} 2 & \mathrm{C} 3 & 1.2(13) & \mathrm{C} 21 & \mathrm{C} 16 & \mathrm{C} 17 & \mathrm{O} 2 & -3.1(11) \\ \mathrm{C} 7 & \mathrm{C} 4 & \mathrm{C} 5 & \mathrm{C} 6 & 178.7(6) & \mathrm{C} 21 & \mathrm{C} 16 & \mathrm{C} 17 & \mathrm{~N} 1 & 176.0(6)\end{array}$

Table A7. Hydrogen Atom Coordinates $\left(\AA \times 10^{4}\right)$ and Isotropic Displacement Parameters $\left(\AA^{2} \times 10^{3}\right)$.

$\begin{array}{lllll}\text { Atom } & \boldsymbol{x} & \boldsymbol{y} & \boldsymbol{z} & \mathbf{U}(\mathbf{e q}) \\ \text { H00A } & 1687 & 2895 & 2090 & 25 \\ \text { H00B } & 423 & 4610 & 2318 & 25 \\ \text { H2 } & 3682 & 3188 & 5149 & 40 \\ \text { H3 } & 3841 & 5478 & 4159 & 34 \\ \text { H5 } & 869 & 1349 & 3058 & 22 \\ \text { H6 } & 712 & -960 & 4057 & 26 \\ \text { H9 } & 4130 & 9960 & 2918 & 23 \\ \text { H11A } & 6618 & 5132 & 2943 & 31 \\ \text { H11B } & 7562 & 5749 & 2292 & 31 \\ \text { H11C } & 5821 & 5034 & 2177 & 31 \\ \text { H12A } & 6892 & 11113 & 3121 & 34 \\ \text { H12B } & 8229 & 9482 & 2918 & 34 \\ \text { H12C } & 7184 & 8811 & 3531 & 34 \\ \text { H13A } & 5457 & 8668 & 1480 & 29 \\ \text { H13B } & 7150 & 9506 & 1673 & 29 \\ \text { H13C } & 5744 & 11033 & 1854 & 29 \\ \text { H18 } & 4098 & 6343 & -216 & 24 \\ \text { H19 } & 3400 & 9527 & -922 & 29 \\ \text { H20 } & 1649 & 12158 & -562 & 27 \\ \text { H21 } & 430 & 11662 & 495 & 21\end{array}$




\section{X-Ray Crystallographic Data for (+)-3g}

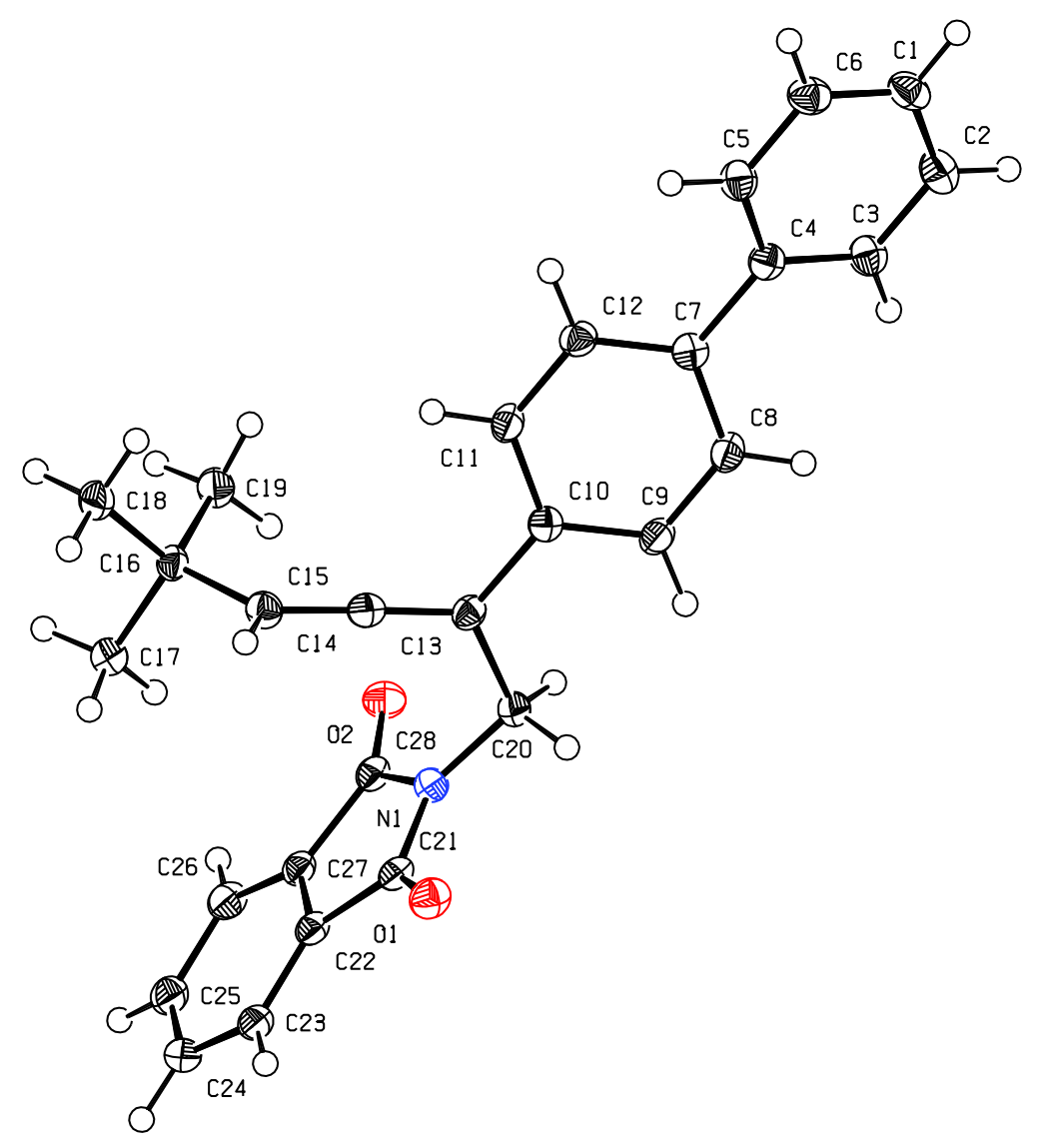

ORTEP view ${ }^{8}$ of (+)-3g, the thermal ellipsoids are drawn at the $50 \%$ probability level.

Database Reference. Crystallographic data have been deposited with the Cambridge Crystallographic Data Centre (CCDC) as supplementary publication no. CCDC-1446561. Data can be obtained free of charge on application to CCDC.

Experimental. A suitable clear, colorless block was selected, mounted in perfluoroalkyl polyether oil on polyimide Micromounts (supplied by MiTeGen) and measured on a Bruker/Nonius Kappa Apex II diffractometer with a Bruker Apex II area detector. The detector type was a CCD area detector. The crystal was kept at 100.0(2) K during data collection. Using Olex $2,{ }^{9}$ the structure was solved with the XS structure solution program ${ }^{10}$ using Direct Methods and refined with the XL refinement package ${ }^{10}$ using Least Squares minimization. The absolute stereochemistry was not determined by X-Ray diffraction.

Table A8. Crystal data and structure refinement. 


\begin{tabular}{|c|c|}
\hline Empirical formula & $\mathrm{C}_{28} \mathrm{H}_{25} \mathrm{NO}_{2}$ \\
\hline Formula weight & 407.49 \\
\hline Temperature/K & $100.0(2)$ \\
\hline Crystal system & orthorhombic \\
\hline Space group & $\mathrm{P} 2{ }_{1}{ }_{1} 2_{1}$ \\
\hline $\mathrm{a} / \AA$ & $5.99770(10)$ \\
\hline $\mathrm{b} / \AA$ & $8.66900(10)$ \\
\hline $\mathrm{c} / \AA$ & $42.0392(7)$ \\
\hline$\alpha /^{\circ}$ & 90 \\
\hline$\beta /{ }^{\circ}$ & 90 \\
\hline$\gamma /{ }^{\circ}$ & 90 \\
\hline Volume $/ \AA^{3}$ & $2185.79(6)$ \\
\hline $\mathrm{Z}$ & 4 \\
\hline$\rho_{\text {calc }} \mathrm{g} / \mathrm{cm}^{3}$ & 1.238 \\
\hline$\mu / \mathrm{mm}^{-1}$ & 0.607 \\
\hline $\mathrm{F}(000)$ & 864.0 \\
\hline Crystal size $/ \mathrm{mm}^{3}$ & $0.14 \times 0.13 \times 0.06$ \\
\hline Radiation & $\mathrm{CuK} \alpha(\lambda=1.54178)$ \\
\hline $2 \Theta$ range for data collection ${ }^{\circ}$ & 4.204 to 133.2 \\
\hline Index ranges & $-7 \leq \mathrm{h} \leq 4,-10 \leq \mathrm{k} \leq 10,-50 \leq 1 \leq 49$ \\
\hline Reflections collected & 28660 \\
\hline Independent reflections & $3862\left[\mathrm{R}_{\mathrm{int}}=0.0430, \mathrm{R}_{\mathrm{sigma}}=0.0225\right]$ \\
\hline Data/restraints/parameters & $3862 / 0 / 283$ \\
\hline Goodness-of-fit on $\mathrm{F}^{2}$ & 1.075 \\
\hline Final $R$ indexes $[\mathrm{I}>=2 \sigma(\mathrm{I})]$ & $\mathrm{R}_{1}=0.0299, \mathrm{wR}_{2}=0.0721$ \\
\hline Final $R$ indexes [all data] & $\mathrm{R}_{1}=0.0317, \mathrm{wR}_{2}=0.0732$ \\
\hline Largest diff. peak/hole / e $\AA^{-3}$ & $0.10 /-0.18$ \\
\hline Flack parameter & $-0.01(10)$ \\
\hline
\end{tabular}

Table A9. Fractional Atomic Coordinates $\left(\times 10^{4}\right)$ and Equivalent Isotropic Displacement Parameters $\left(\AA^{2} \times 10^{3}\right)$. $U_{\text {eq }}$ is defined as $1 / 3$ of the trace of the orthogonalised $U_{\text {IJ }}$ tensor.

\begin{tabular}{lllll} 
Atom & \multicolumn{1}{c}{$\boldsymbol{x}$} & \multicolumn{1}{c}{$\boldsymbol{y}$} & \multicolumn{1}{c}{$\boldsymbol{z}$} & $\mathbf{U}(\mathbf{e q})$ \\
$\mathrm{O} 1$ & $-3094(2)$ & $7697.6(15)$ & $4188.9(3)$ & $25.6(3)$ \\
$\mathrm{O} 2$ & $1915(2)$ & $4031.2(15)$ & $4506.2(3)$ & $25.3(3)$ \\
$\mathrm{N} 1$ & $-201(3)$ & $6009.7(17)$ & $4292.9(3)$ & $19.6(3)$ \\
$\mathrm{C} 1$ & $9731(3)$ & $5803(2)$ & $2146.3(4)$ & $25.9(4)$ \\
C2 & $10305(4)$ & $6483(2)$ & $2433.8(5)$ & $27.0(4)$ \\
C3 & $8846(3)$ & $6403(2)$ & $2689.7(4)$ & $23.4(4)$
\end{tabular}




$\begin{array}{lllll}\text { C4 } & 6793(3) & 5662(2) & 2663.6(4) & 20.4(4) \\ \text { C5 } & 6232(3) & 5001(2) & 2370.2(4) & 23.3(4) \\ \text { C6 } & 7706(4) & 5070(2) & 2115.5(4) & 25.9(4) \\ \text { C7 } & 5239(3) & 5565(2) & 2939.1(4) & 19.4(4) \\ \text { C8 } & 5266(3) & 6675(2) & 3180.4(4) & 22.7(4) \\ \text { C9 } & 3809(3) & 6601(2) & 3434.8(4) & 22.1(4) \\ \text { C10 } & 2237(3) & 5422(2) & 3459.8(4) & 18.2(4) \\ \text { C11 } & 2211(3) & 4303(2) & 3219.6(4) & 21.1(4) \\ \text { C12 } & 3684(3) & 4372(2) & 2967.0(4) & 21.5(4) \\ \text { C13 } & 700(3) & 5310(2) & 3734.5(4) & 18.6(4) \\ \text { C14 } & -1025(3) & 4379(2) & 3740.6(4) & 19.8(4) \\ \text { C15 } & -2751(3) & 3465(2) & 3752.4(4) & 21.1(4) \\ \text { C16 } & -2746(3) & 1804(2) & 3869.3(4) & 18.8(4) \\ \text { C17 } & -3826(3) & 1754(2) & 4200.4(4) & 24.4(4) \\ \text { C18 } & -4150(3) & 832(2) & 3640.3(4) & 25.0(4) \\ \text { C19 } & -384(3) & 1167(2) & 3884.1(5) & 23.5(4) \\ \text { C20 } & 1201(3) & 6337(2) & 4019.0(4) & 21.2(4) \\ \text { C21 } & -2236(3) & 6719(2) & 4354.6(4) & 19.3(4) \\ \text { C22 } & -3046(3) & 6044(2) & 4659.4(4) & 18.8(4) \\ \text { C23 } & -4919(3) & 6393(2) & 4835.9(4) & 21.5(4) \\ \text { C24 } & -5220(4) & 5569(2) & 5118.9(4) & 25.2(4) \\ \text { C25 } & -3693(4) & 4464(2) & 5216.6(4) & 26.1(4) \\ \text { C26 } & -1806(3) & 4123(2) & 5037.0(4) & 23.5(4) \\ \text { C27 } & -1520(3) & 4936(2) & 4756.5(4) & 18.9(4) \\ \text { C28 } & 298(3) & 4866(2) & 4516.7(4) & 19.3(4)\end{array}$

Table A10. Anisotropic Displacement Parameters $\left(\AA^{2} \times 10^{3}\right)$. The Anisotropic displacement factor exponent takes the form: $-2 \pi^{2}\left[h^{2} a * 2 U_{11}+2 h k a * b * U_{12}+\ldots\right]$.

\begin{tabular}{lllllll}
\multicolumn{1}{c}{ Atom } & \multicolumn{1}{c}{$\mathbf{U}_{\mathbf{1 1}}$} & \multicolumn{1}{c}{$\mathbf{U}_{\mathbf{2 2}}$} & \multicolumn{1}{c}{$\mathbf{U}_{\mathbf{3 3}}$} & \multicolumn{1}{c}{$\mathbf{U}_{\mathbf{2 3}}$} & \multicolumn{1}{c}{$\mathbf{U}_{\mathbf{1 3}}$} & $\mathbf{U}_{\mathbf{1 2}}$ \\
$\mathrm{O} 1$ & $30.4(8)$ & $21.0(7)$ & $25.4(7)$ & $3.5(6)$ & $-1.0(6)$ & $2.8(6)$ \\
$\mathrm{O} 2$ & $24.3(7)$ & $24.4(7)$ & $27.1(7)$ & $-1.0(6)$ & $0.3(6)$ & $5.0(6)$ \\
$\mathrm{N} 1$ & $22.0(8)$ & $18.5(7)$ & $18.4(7)$ & $0.0(6)$ & $2.3(6)$ & $-1.0(7)$ \\
$\mathrm{C} 1$ & $26.9(10)$ & $27(1)$ & $23.8(9)$ & $5.9(8)$ & $6.3(8)$ & $5.7(9)$ \\
C2 & $21.7(10)$ & $30.3(10)$ & $29.1(10)$ & $5.8(8)$ & $1.7(8)$ & $0.5(9)$ \\
C3 & $24.5(10)$ & $25(1)$ & $20.7(9)$ & $2.4(8)$ & $-0.2(8)$ & $-0.7(8)$ \\
C4 & $23(1)$ & $17.4(8)$ & $20.7(9)$ & $3.3(7)$ & $-0.2(7)$ & $2.5(8)$ \\
C5 & $23.9(10)$ & $23.6(9)$ & $22.2(9)$ & $2.3(8)$ & $0.2(8)$ & $-1.2(8)$ \\
C6 & $31.6(11)$ & $25.9(10)$ & $20.1(9)$ & $1.3(8)$ & $2.3(8)$ & $3.9(9)$
\end{tabular}




$\begin{array}{lllllll}\text { C7 } & 20.9(9) & 19.9(9) & 17.3(8) & 2.0(7) & -1.7(7) & 1.3(8) \\ \text { C8 } & 26.9(10) & 18.5(9) & 22.8(9) & 0.8(8) & -0.7(8) & -4.7(9) \\ \text { C9 } & 29(1) & 17.0(9) & 20.3(9) & -1.4(7) & 0.5(8) & -3.5(8) \\ \text { C10 } & 19.7(9) & 17.3(9) & 17.5(8) & 2.7(7) & -1.2(7) & 1.4(8) \\ \text { C11 } & 24.4(10) & 18.4(9) & 20.5(9) & 0.0(7) & -1.1(7) & -5.2(8) \\ \text { C12 } & 25.7(10) & 20.8(9) & 18.0(8) & -2.2(7) & -0.6(7) & -1.2(8) \\ \text { C13 } & 22.2(10) & 14.9(9) & 18.8(8) & 1.1(7) & -0.9(7) & 0.1(8) \\ \text { C14 } & 23.3(10) & 18.8(9) & 17.1(8) & 0.9(7) & -0.1(7) & 2.9(8) \\ \text { C15 } & 18.0(9) & 22.0(9) & 23.4(9) & 0.7(8) & -0.5(8) & 1.2(8) \\ \text { C16 } & 16.4(9) & 17.6(9) & 22.3(9) & -0.8(7) & -0.3(7) & -2.5(8) \\ \text { C17 } & 25.6(10) & 23.2(10) & 24.5(9) & -1.2(8) & 3.8(8) & -2.2(9) \\ \text { C18 } & 21.9(10) & 27.4(10) & 25.7(9) & -4.5(8) & 1.8(8) & -6.3(9) \\ \text { C19 } & 20.1(10) & 21.4(9) & 29(1) & 2.9(8) & 0.8(8) & 1.5(8) \\ \text { C20 } & 24.0(9) & 19.4(9) & 20.0(9) & -1.3(7) & 4.2(8) & -4.1(8) \\ \text { C21 } & 23.2(10) & 15.0(8) & 19.5(8) & -2.6(7) & -1.6(8) & -0.8(8) \\ \text { C22 } & 21.7(9) & 16.0(9) & 18.8(8) & -2.9(7) & -1.4(7) & -1.7(8) \\ \text { C23 } & 20.6(10) & 18.8(9) & 25.1(9) & -3.2(7) & 0.4(8) & -0.6(8) \\ \text { C24 } & 27.8(11) & 22.4(9) & 25.3(9) & -4.6(8) & 7.4(8) & -2.3(9) \\ \text { C25 } & 37.5(11) & 20.6(9) & 20.1(9) & 0.8(8) & 4.9(8) & -4.0(9) \\ \text { C26 } & 30.7(11) & 19.3(9) & 20.7(9) & -0.4(7) & -0.6(8) & -0.4(8) \\ \text { C27 } & 21.8(10) & 16.4(8) & 18.6(8) & -2.2(7) & -1.1(7) & -2.8(7) \\ \text { C28 } & 21.3(10) & 17.2(9) & 19.6(8) & -2.2(7) & -1.9(7) & -2.3(8) \\ & & & & & \end{array}$

Table A11. Bond Lengths.

\begin{tabular}{|c|c|c|c|c|c|}
\hline Atom & Atom & Length/Å & Atom & Atom & Length/Å \\
\hline $\mathrm{O} 1$ & $\mathrm{C} 21$ & $1.213(2)$ & $\mathrm{C} 10$ & C13 & $1.481(2)$ \\
\hline $\mathrm{O} 2$ & C28 & $1.211(2)$ & C11 & $\mathrm{C} 12$ & $1.383(3)$ \\
\hline N1 & C20 & $1.454(2)$ & $\mathrm{C} 13$ & $\mathrm{C} 14$ & $1.312(3)$ \\
\hline N1 & $\mathrm{C} 21$ & $1.391(2)$ & C13 & C20 & $1.522(2)$ \\
\hline N1 & C28 & $1.399(2)$ & C14 & $\mathrm{C} 15$ & $1.305(3)$ \\
\hline $\mathrm{C} 1$ & $\mathrm{C} 2$ & $1.388(3)$ & $\mathrm{C} 15$ & $\mathrm{C} 16$ & $1.521(3)$ \\
\hline $\mathrm{C} 1$ & C6 & $1.377(3)$ & C16 & C17 & $1.536(3)$ \\
\hline $\mathrm{C} 2$ & $\mathrm{C} 3$ & $1.388(3)$ & $\mathrm{C} 16$ & C18 & $1.531(3)$ \\
\hline $\mathrm{C} 3$ & $\mathrm{C} 4$ & $1.393(3)$ & $\mathrm{C} 16$ & C19 & $1.522(3)$ \\
\hline $\mathrm{C} 4$ & $\mathrm{C} 5$ & $1.401(3)$ & $\mathrm{C} 21$ & $\mathrm{C} 22$ & $1.490(3)$ \\
\hline $\mathrm{C} 4$ & $\mathrm{C} 7$ & $1.489(2)$ & $\mathrm{C} 22$ & $\mathrm{C} 23$ & $1.380(3)$ \\
\hline $\mathrm{C} 5$ & C6 & $1.389(3)$ & $\mathrm{C} 22$ & $\mathrm{C} 27$ & $1.388(3)$ \\
\hline $\mathrm{C} 7$ & $\mathrm{C} 8$ & $1.398(3)$ & $\mathrm{C} 23$ & $\mathrm{C} 24$ & $1.399(3)$ \\
\hline $\mathrm{C} 7$ & $\mathrm{C} 12$ & $1.398(3)$ & $\begin{array}{l}\mathrm{C} 24 \\
\mathrm{~S}-35\end{array}$ & $\mathrm{C} 25$ & $1.387(3)$ \\
\hline
\end{tabular}




$\begin{array}{llllll}\mathrm{C} 8 & \mathrm{C} 9 & 1.383(3) & \mathrm{C} 25 & \mathrm{C} 26 & 1.393(3) \\ \mathrm{C} 9 & \mathrm{C} 10 & 1.395(3) & \mathrm{C} 26 & \mathrm{C} 27 & 1.384(3) \\ \mathrm{C} 10 & \mathrm{C} 11 & 1.400(2) & \mathrm{C} 27 & \mathrm{C} 28 & 1.486(3)\end{array}$

Table A12. Bond Angles.

\begin{tabular}{|c|c|c|c|}
\hline Atom & Atom & Atom & Angle ${ }^{\circ}$ \\
\hline $\mathrm{C} 21$ & N1 & $\mathrm{C} 20$ & $124.68(15)$ \\
\hline 21 & N1 & $\mathrm{C} 28$ & $112.03(14)$ \\
\hline 28 & N1 & $\mathrm{C} 20$ & $123.19(15)$ \\
\hline C6 & $\mathrm{C} 1$ & $\mathrm{C} 2$ & $119.79(18)$ \\
\hline $\mathrm{C1}$ & $\mathrm{C} 2$ & C3 & $119.79(19)$ \\
\hline $\mathrm{C} 2$ & $\mathrm{C} 3$ & $\mathrm{C} 4$ & $121.28(18)$ \\
\hline $\mathrm{C} 3$ & $\mathrm{C} 4$ & C5 & $118.05(17)$ \\
\hline $\mathrm{C} 3$ & $\mathrm{C} 4$ & C7 & $121.20(16)$ \\
\hline $\mathrm{C} 5$ & $\mathrm{C} 4$ & C7 & $120.75(17)$ \\
\hline C6 & $\mathrm{C} 5$ & $\mathrm{C} 4$ & $120.53(19)$ \\
\hline $\mathrm{C} 1$ & C6 & $\mathrm{C} 5$ & $120.56(18)$ \\
\hline $\mathrm{C} 8$ & C7 & $\mathrm{C} 4$ & $121.19(17)$ \\
\hline $\mathrm{C} 12$ & C7 & $\mathrm{C} 4$ & $121.64(16)$ \\
\hline $\mathrm{C} 12$ & $\mathrm{C} 7$ & $\mathrm{C} 8$ & $117.17(16)$ \\
\hline C9 & C8 & C7 & $121.45(17)$ \\
\hline $\mathrm{C} 8$ & C9 & $\mathrm{C} 10$ & $121.29(17)$ \\
\hline C9 & $\mathrm{C} 10$ & $\mathrm{C} 11$ & $117.47(16)$ \\
\hline C9 & $\mathrm{C} 10$ & $\mathrm{C} 13$ & $121.86(15)$ \\
\hline C11 & $\mathrm{C} 10$ & $\mathrm{C} 13$ & $120.63(16)$ \\
\hline $\mathrm{C} 12$ & C11 & $\mathrm{C} 10$ & $121.10(18)$ \\
\hline C11 & $\mathrm{C} 12$ & C7 & $121.51(17)$ \\
\hline C10 & $\mathrm{C} 13$ & $\mathrm{C} 20$ & $116.84(15)$ \\
\hline C14 & $\mathrm{C} 13$ & $\mathrm{C} 10$ & $123.14(16)$ \\
\hline C14 & $\mathrm{C} 13$ & $\mathrm{C} 20$ & $120.02(16)$ \\
\hline 15 & C14 & $\mathrm{C} 13$ & 78. \\
\hline
\end{tabular}

\begin{tabular}{|c|c|c|c|}
\hline Atom & Atom & Atom & Angle ${ }^{\circ}$ \\
\hline C14 & C15 & $\mathrm{C} 16$ & $125.85(17)$ \\
\hline 615 & C16 & $\mathrm{C} 17$ & $108.57(15)$ \\
\hline $\mathrm{C} 15$ & $\mathrm{C} 16$ & $\mathrm{C} 18$ & $108.46(15)$ \\
\hline C15 & C16 & C19 & $110.97(15)$ \\
\hline C18 & C16 & $\mathrm{C} 17$ & $108.81(15)$ \\
\hline C19 & C16 & $\mathrm{C} 17$ & $110.22(15)$ \\
\hline C19 & C16 & $\mathrm{C} 18$ & $109.76(15)$ \\
\hline $\mathrm{N} 1$ & $\mathrm{C} 20$ & $\mathrm{C} 13$ & $113.19(15)$ \\
\hline $\mathrm{O} 1$ & $\mathrm{C} 21$ & N1 & $125.07(17)$ \\
\hline $\mathrm{D} 1$ & $\mathrm{C} 21$ & $\mathrm{C} 22$ & $129.06(18)$ \\
\hline N1 & $\mathrm{C} 21$ & $\mathrm{C} 22$ & $105.86(15)$ \\
\hline $\mathrm{C} 23$ & $\mathrm{C} 22$ & $\mathrm{C} 21$ & $129.95(17)$ \\
\hline $\mathrm{C} 23$ & $\mathrm{C} 22$ & $\mathrm{C} 27$ & $122.01(16)$ \\
\hline $\mathrm{C} 27$ & $\mathrm{C} 22$ & $\mathrm{C} 21$ & $108.02(16)$ \\
\hline $\mathrm{C} 22$ & $\mathrm{C} 23$ & $\mathrm{C} 24$ & 116.77(18) \\
\hline $\mathrm{C} 25$ & $\mathrm{C} 24$ & $\mathrm{C} 23$ & $121.27(19)$ \\
\hline C24 & $\mathrm{C} 25$ & $\mathrm{C} 26$ & $121.50(18)$ \\
\hline $\mathrm{C} 27$ & $\mathrm{C} 26$ & $\mathrm{C} 25$ & $117.03(18)$ \\
\hline $\mathrm{C} 22$ & $\mathrm{C} 27$ & $\mathrm{C} 28$ & $108.20(15)$ \\
\hline $\mathrm{C} 26$ & $\mathrm{C} 27$ & $\mathrm{C} 22$ & $121.41(18)$ \\
\hline $\mathrm{C} 26$ & $\mathrm{C} 27$ & $\mathrm{C} 28$ & $130.38(18)$ \\
\hline $\mathrm{O} 2$ & $\mathrm{C} 28$ & N1 & $124.76(17)$ \\
\hline $\mathrm{O} 2$ & $\mathrm{C} 28$ & $\mathrm{C} 27$ & $129.54(17)$ \\
\hline N1 & $\mathrm{C} 28$ & $\mathrm{C} 27$ & $105.70(15)$ \\
\hline
\end{tabular}

Table A13. Torsion Angles.

\begin{tabular}{rrrrl}
$\mathbf{A}$ & $\mathbf{B}$ & $\mathbf{C}$ & $\mathbf{D}$ & \multicolumn{1}{c}{ Angle $^{\circ}$} \\
$\mathrm{O} 1$ & $\mathrm{C} 21$ & $\mathrm{C} 22$ & $\mathrm{C} 23$ & $-2.6(3)$ \\
$\mathrm{O} 1$ & $\mathrm{C} 21$ & $\mathrm{C} 22$ & $\mathrm{C} 27$ & $178.57(18)$ \\
$\mathrm{N} 1$ & $\mathrm{C} 21$ & $\mathrm{C} 22$ & $\mathrm{C} 23$ & $176.47(18)$ \\
$\mathrm{N} 1$ & $\mathrm{C} 21$ & $\mathrm{C} 22$ & $\mathrm{C} 27$ & $-2.34(19)$
\end{tabular}

\begin{tabular}{rrrrl} 
A & $\mathbf{B}$ & $\mathbf{C}$ & $\mathbf{D}$ & \multicolumn{1}{c}{ Angle $^{\circ}$} \\
$\mathrm{C} 13$ & $\mathrm{C} 10$ & $\mathrm{C} 11$ & $\mathrm{C} 12$ & $-178.05(18)$ \\
$\mathrm{C} 14$ & $\mathrm{C} 13$ & $\mathrm{C} 20$ & $\mathrm{~N} 1$ & $7.6(2)$ \\
$\mathrm{C} 14$ & $\mathrm{C} 15$ & $\mathrm{C} 16$ & $\mathrm{C} 17$ & $105.0(2)$ \\
$\mathrm{C} 14$ & $\mathrm{C} 15$ & $\mathrm{C} 16$ & $\mathrm{C} 18$ & $-136.87(19)$
\end{tabular}




\begin{tabular}{|c|c|c|c|c|c|c|c|c|}
\hline $\mathrm{C} 2$ & $\mathrm{C} 3$ & $\mathrm{C} 4$ & $-0.7(3)$ & $\mathrm{C} 14$ & $\mathrm{C} 15$ & $\mathrm{C} 16$ & C19 & $-16.2(2)$ \\
\hline $\mathrm{C} 1$ & C6 & $\mathrm{C} 5$ & $-0.2(3)$ & $\mathrm{C} 20$ & N1 & $\mathrm{C} 21$ & $\mathrm{O} 1$ & $-0.1(3)$ \\
\hline $\mathrm{C} 2$ & $\mathrm{C} 4$ & $\mathrm{C} 5$ & $-0.2(3)$ & $\mathrm{C} 20$ & N1 & $\mathrm{C} 21$ & $\mathrm{C} 22$ & $-179.21(15)$ \\
\hline C3 & $\mathrm{C} 4$ & C7 & $179.47(18)$ & $\mathrm{C} 20$ & N1 & $\mathrm{C} 28$ & $\mathrm{O} 2$ & $-0.5(3)$ \\
\hline C3 & $\mathrm{C} 5$ & C6 & $0.9(3)$ & $\mathrm{C} 20$ & N1 & $\mathrm{C} 28$ & $\mathrm{C} 27$ & $179.01(15)$ \\
\hline $\mathrm{C} 3$ & C7 & $\mathrm{C} 8$ & $26.8(3)$ & $\mathrm{C} 21$ & N1 & $\mathrm{C} 20$ & $\mathrm{C} 13$ & $-90.6(2)$ \\
\hline $\mathrm{C} 3$ & $\mathrm{C} 7$ & $\mathrm{C} 12$ & $-153.66(18)$ & $\mathrm{C} 21$ & N1 & $\mathrm{C} 28$ & $\mathrm{O} 2$ & 176.11(17) \\
\hline $\mathrm{C} 4$ & C6 & $\mathrm{C} 1$ & $-0.7(3)$ & $\mathrm{C} 21$ & N1 & $\mathrm{C} 28$ & $\mathrm{C} 27$ & $-4.37(19)$ \\
\hline $\mathrm{C} 4$ & $\mathrm{C} 8$ & C9 & $179.21(17)$ & $\mathrm{C} 21$ & $\mathrm{C} 22$ & $\mathrm{C} 23$ & $\mathrm{C} 24$ & $-178.89(18)$ \\
\hline $\mathrm{C} 4$ & $\mathrm{C} 12$ & C11 & $-178.60(17)$ & $\mathrm{C} 21$ & $\mathrm{C} 22$ & $\mathrm{C} 27$ & $\mathrm{C} 26$ & $178.87(17)$ \\
\hline $\mathrm{C} 5$ & C7 & $\mathrm{C} 8$ & $-153.53(18)$ & $\mathrm{C} 21$ & $\mathrm{C} 22$ & $\mathrm{C} 27$ & $\mathrm{C} 28$ & $-0.24(19)$ \\
\hline C5 & $\mathrm{C} 7$ & $\mathrm{C} 12$ & $26.0(3)$ & $\mathrm{C} 22$ & $\mathrm{C} 23$ & $\mathrm{C} 24$ & $\mathrm{C} 25$ & $0.4(3)$ \\
\hline C6 & $\mathrm{C} 2$ & C3 & $0.9(3)$ & $\mathrm{C} 22$ & $\mathrm{C} 27$ & $\mathrm{C} 28$ & $\mathrm{O} 2$ & $-177.80(18)$ \\
\hline $\mathrm{C} 7$ & $\mathrm{C} 5$ & C6 & $-178.79(17)$ & $\mathrm{C} 22$ & $\mathrm{C} 27$ & $\mathrm{C} 28$ & N1 & $2.72(19)$ \\
\hline C7 & C9 & $\mathrm{C} 10$ & $-0.6(3)$ & $\mathrm{C} 23$ & $\mathrm{C} 22$ & $\mathrm{C} 27$ & $\mathrm{C} 26$ & $-0.1(3)$ \\
\hline $\mathrm{C} 7$ & $\mathrm{C} 12$ & C11 & $1.0(3)$ & $\mathrm{C} 23$ & $\mathrm{C} 22$ & $\mathrm{C} 27$ & $\mathrm{C} 28$ & $-179.16(16)$ \\
\hline $\mathrm{C} 8$ & $\mathrm{C} 10$ & $\mathrm{C} 11$ & $0.9(3)$ & $\mathrm{C} 23$ & $\mathrm{C} 24$ & $\mathrm{C} 25$ & $\mathrm{C} 26$ & $-0.3(3)$ \\
\hline C9 & $\mathrm{C} 10$ & $\mathrm{C} 13$ & $178.63(17)$ & $\mathrm{C} 24$ & $\mathrm{C} 25$ & $\mathrm{C} 26$ & $\mathrm{C} 27$ & $0.0(3)$ \\
\hline $\mathrm{C} 10$ & $\mathrm{C} 11$ & $\mathrm{C} 12$ & $-0.3(3)$ & $\mathrm{C} 25$ & $\mathrm{C} 26$ & $\mathrm{C} 27$ & $\mathrm{C} 22$ & $0.2(3)$ \\
\hline $\mathrm{C} 10$ & $\mathrm{C} 13$ & C14 & $168.39(18)$ & $\mathrm{C} 25$ & $\mathrm{C} 26$ & $\mathrm{C} 27$ & $\mathrm{C} 28$ & $179.04(18)$ \\
\hline $\mathrm{C} 10$ & $\mathrm{C} 13$ & $\mathrm{C} 20$ & $-11.7(3)$ & $\mathrm{C} 26$ & $\mathrm{C} 27$ & $\mathrm{C} 28$ & $\mathrm{O} 2$ & $3.2(3)$ \\
\hline $\mathrm{C} 10$ & $\mathrm{C} 12$ & C7 & $-0.7(3)$ & $\mathrm{C} 26$ & $\mathrm{C} 27$ & $\mathrm{C} 28$ & N1 & $-176.28(18)$ \\
\hline $\mathrm{C} 10$ & $\mathrm{C} 20$ & N1 & $-172.30(15)$ & $\mathrm{C} 27$ & $\mathrm{C} 22$ & $\mathrm{C} 23$ & $\mathrm{C} 24$ & $-0.2(3)$ \\
\hline $\mathrm{C} 10$ & $\mathrm{C} 13$ & C14 & $-13.9(3)$ & $\mathrm{C} 28$ & N1 & $\mathrm{C} 20$ & $\mathrm{C} 13$ & $85.6(2)$ \\
\hline $\mathrm{C} 11$ & $\mathrm{C} 13$ & $\mathrm{C} 20$ & $165.93(17)$ & $\mathrm{C} 28$ & N1 & $\mathrm{C} 21$ & $\mathrm{O} 1$ & $-176.63(18)$ \\
\hline $\mathrm{C} 12$ & $\mathrm{C} 8$ & C9 & $-0.3(3)$ & $\mathrm{C} 28$ & N1 & $\mathrm{C} 21$ & $\mathrm{C} 22$ & $4.23(19)$ \\
\hline
\end{tabular}

Table A14. Hydrogen Atom Coordinates $\left(\AA \times 10^{4}\right)$ and Isotropic Displacement Parameters $\left(\AA^{2} \times 10^{3}\right)$.

\begin{tabular}{lllll}
\multicolumn{1}{r}{ Atom } & \multicolumn{1}{c}{$\boldsymbol{x}$} & \multicolumn{1}{c}{$\boldsymbol{y}$} & $\boldsymbol{z}$ & $\mathbf{U}(\mathbf{e q})$ \\
H1 & 10732 & 5843 & 1971 & 31 \\
H2 & 11691 & 7003 & 2455 & 32 \\
H3 & 9256 & 6861 & 2886 & 28 \\
H5 & 4833 & 4502 & 2345 & 28 \\
H6 & 7313 & 4607 & 1919 & 31 \\
H8 & 6309 & 7498 & 3169 & 27 \\
H9 & 3881 & 7369 & 3596 & 27 \\
H11 & 1163 & 3482 & 3230 & 25 \\
H12 & 3638 & 3590 & 2809 & 26 \\
H15 & -4135 & 3873 & 3682 & 25 \\
& & & & S-37
\end{tabular}




$\begin{array}{lllll}\text { H17A } & -2852 & 2272 & 4354 & 37 \\ \text { H17B } & -4045 & 678 & 4265 & 37 \\ \text { H17C } & -5271 & 2281 & 4194 & 37 \\ \text { H18A } & -5667 & 1249 & 3631 & 38 \\ \text { H18B } & -4199 & -237 & 3716 & 38 \\ \text { H18C } & -3481 & 863 & 3428 & 38 \\ \text { H19A } & 237 & 1112 & 3669 & 35 \\ \text { H19B } & -410 & 132 & 3978 & 35 \\ \text { H19C } & 544 & 1847 & 4015 & 35 \\ \text { H20A } & 2783 & 6202 & 4080 & 25 \\ \text { H20B } & 992 & 7428 & 3956 & 25 \\ \text { H23 } & -5955 & 7155 & 4769 & 26 \\ \text { H24 } & -6494 & 5770 & 5247 & 30 \\ \text { H25 } & -3941 & 3928 & 5411 & 31 \\ \text { H26 } & -761 & 3366 & 5104 & 28\end{array}$

\section{X-Ray Crystallographic Data for (+)-3h}

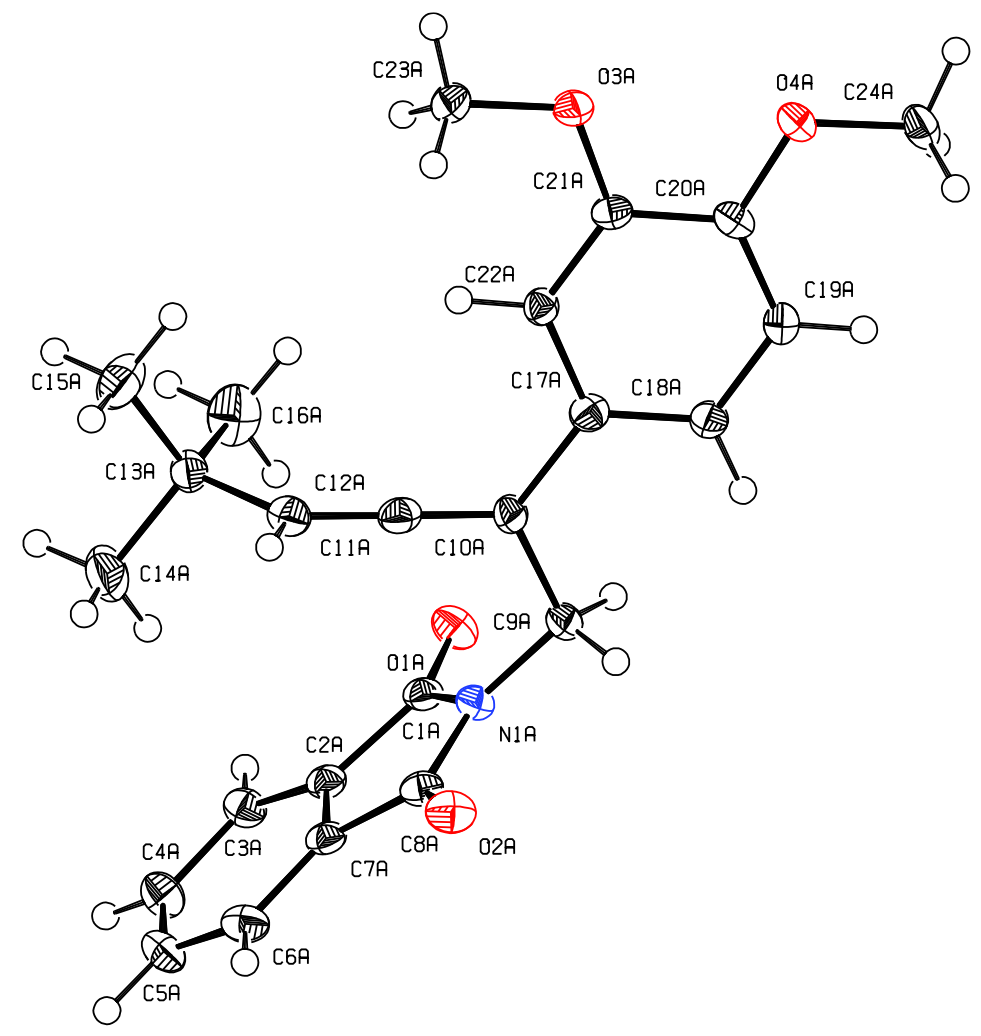

ORTEP view ${ }^{8}$ of (+)-3h, the thermal ellipsoids are drawn at the $50 \%$ probability level. 
Database Reference. Crystallographic data have been deposited with the Cambridge Crystallographic Data Centre (CCDC) as supplementary publication no. CCDC-1437269. Data can be obtained free of charge on application to CCDC.

Experimental. A suitable clear, colorless block was selected, mounted in perfluoroalkyl polyether oil on polyimide Micromounts (supplied by MiTeGen) and measured on a Bruker/Nonius Kappa Apex II diffractometer with a Bruker Apex II area detector. The detector type was a CCD area detector. The crystal was kept at 100.0(2) K during data collection. Using Olex2, ${ }^{9}$ the structure was solved with the XS structure solution program ${ }^{10}$ using Direct Methods and refined with the XL refinement package ${ }^{10}$ using Least Squares minimization. The absolute stereochemistry was not determined by X-Ray diffraction.

Table A15. Crystal data and structure refinement.

\begin{tabular}{|c|c|}
\hline Empirical formula & $\mathrm{C}_{24} \mathrm{H}_{25} \mathrm{NO}_{4}$ \\
\hline Formula weight & 391.45 \\
\hline Temperature/K & 100.0 \\
\hline Crystal system & triclinic \\
\hline Space group & P1 \\
\hline $\mathrm{a} / \AA$ & $12.7971(3)$ \\
\hline $\mathrm{b} / \mathrm{A}$ & $12.8656(3)$ \\
\hline$c / \AA$ & $14.0933(3)$ \\
\hline$\alpha{ }^{\circ}$ & $88.9680(10)$ \\
\hline$\beta /{ }^{\circ}$ & $86.1080(10)$ \\
\hline$\gamma /{ }^{\circ}$ & $66.5820(10)$ \\
\hline Volume $/ \AA^{3}$ & $2124.21(8)$ \\
\hline $\mathrm{Z}$ & 4 \\
\hline$\rho_{\text {calc }} \mathrm{g} / \mathrm{cm}^{3}$ & 1.224 \\
\hline$\mu / \mathrm{mm}^{-1}$ & 0.671 \\
\hline$F(000)$ & 832.0 \\
\hline Crystal size $/ \mathrm{mm}^{3}$ & $0.15 \times 0.13 \times 0.07$ \\
\hline Radiation & $\mathrm{CuK} \alpha(\lambda=1.54178)$ \\
\hline $2 \Theta$ range for data collection ${ }^{\circ}$ & 6.286 to 133.496 \\
\hline Index ranges & $-11 \leq \mathrm{h} \leq 14,-14 \leq \mathrm{k} \leq 15,-16 \leq 1 \leq 16$ \\
\hline Reflections collected & 36886 \\
\hline Independent reflections & $11081\left[R_{\text {int }}=0.0261, R_{\text {sigma }}=0.0255\right]$ \\
\hline Data/restraints/parameters & $11081 / 3 / 1065$ \\
\hline Goodness-of-fit on $\mathrm{F}^{2}$ & 1.020 \\
\hline
\end{tabular}


Final R indexes $[\mathrm{I}>=2 \sigma(\mathrm{I})]$

Final $R$ indexes [all data]

Largest diff. peak/hole / e $\AA^{-3}$

Flack parameter
$\mathrm{R}_{1}=0.0307, \mathrm{wR}_{2}=0.0827$

$\mathrm{R}_{1}=0.0313, \mathrm{wR}_{2}=0.0833$

$0.27 /-0.20$

$0.12(6)$

Table A16. Fractional Atomic Coordinates $\left(\times 10^{4}\right)$ and Equivalent Isotropic Displacement Parameters $\left(\AA^{2} \times 10^{3}\right)$. $U_{\text {eq }}$ is defined as $1 / 3$ of the trace of the orthogonalised $U_{\text {IJ }}$ tensor.

$\begin{array}{lllll}\text { Atom } & \boldsymbol{x} & \boldsymbol{y} & \boldsymbol{z} & \mathbf{U}(\mathbf{e q}) \\ \mathrm{O} 1 \mathrm{C} & 3936.0(16) & 2732.8(15) & 4309.0(12) & 30.1(4) \\ \text { O2C } & 4439.5(15) & 5154.2(14) & 6397.9(12) & 27.6(4) \\ \text { O3C } & -1422.4(14) & 9066.3(14) & 4621.7(11) & 22.6(4) \\ \text { O4C } & -471.1(15) & 9954.7(14) & 3356.9(12) & 24.6(4) \\ \text { N1C } & 4032.5(17) & 4170.1(17) & 5214.9(14) & 20.5(4) \\ \text { C1C } & 4209(2) & 3059(2) & 5018.7(16) & 20.7(5) \\ \text { C2C } & 4798(2) & 2383(2) & 5836.8(16) & 18.7(5) \\ \text { C3C } & 5152(2) & 1235(2) & 6016.7(17) & 24.2(5) \\ \text { C4C } & 5676(2) & 844(2) & 6860.7(17) & 24.8(5) \\ \text { C5C } & 5828(2) & 1571(2) & 7499.2(17) & 23.0(5) \\ \text { C6C } & 5454(2) & 2733(2) & 7318.8(17) & 21.3(5) \\ \text { C7C } & 4944(2) & 3110(2) & 6474.9(17) & 19.6(5) \\ \text { C8C } & 4465(2) & 4279(2) & 6074.3(17) & 20.6(5) \\ \text { C9C } & 3410(2) & 5133(2) & 4629.4(17) & 23.1(5) \\ \text { C10C } & 2217(2) & 5831(2) & 5065.1(17) & 21.7(5) \\ \text { C11C } & 1896(2) & 5575.5(19) & 5906.4(17) & 24.2(5) \\ \text { C12C } & 1645(2) & 5320.8(19) & 6769.0(16) & 26.0(5) \\ \text { C13C } & 1040(2) & 4548(2) & 7041.1(17) & 26.1(6) \\ \text { C14C } & -81(3) & 5241(3) & 7599(2) & 48.3(8) \\ \text { C15C } & 810(3) & 4021(3) & 6166(2) & 45.3(7) \\ \text { C16C } & 1798(3) & 3609(2) & 7674(2) & 44.6(7) \\ \text { C17C } & 1521(2) & 6866(2) & 4545.8(16) & 18.0(5) \\ \text { C18C } & 366(2) & 7461.4(19) & 4839.4(16) & 18.6(5) \\ \text { C19C } & -285(2) & 8467.7(19) & 4415.8(15) & 18.3(5) \\ \text { C20C } & 230(2) & 8930(2) & 3708.9(16) & 19.0(5) \\ \text { C21C } & 1354(2) & 8331(2) & 3394.5(17) & 20.5(5) \\ \text { C22C } & 1995(2) & 7294(2) & 3801.7(16) & 20.5(5) \\ \text { C23C } & -2003(2) & 8524(2) & 5220.5(17) & 25.1(6) \\ \text { C24C } & 28(2) & 10451(2) & 2644.3(19) & 28.7(6) \\ \text { O1A } & 5734.3(15) & 7488.8(14) & -128.2(12) & 27.2(4)\end{array}$




\begin{tabular}{|c|c|c|c|c|}
\hline $\mathrm{O} 2 \mathrm{~A}$ & $2034.4(15)$ & $8627.6(15)$ & $1066.6(12)$ & $26.9(4)$ \\
\hline O3A & $6548.4(14)$ & $4608.4(13)$ & $4790.0(11)$ & $22.0(4)$ \\
\hline O4A & $6725.4(15)$ & $2710.7(14)$ & $4057.9(12)$ & $24.6(4)$ \\
\hline N1A & 3949.7(18) & 7798.7(16) & $562.9(13)$ & $19.9(4)$ \\
\hline C1A & $4744(2)$ & $8114(2)$ & $54.6(16)$ & $19.4(5)$ \\
\hline $\mathrm{C} 2 \mathrm{~A}$ & $4121(2)$ & $9323(2)$ & $-203.0(16)$ & $19.2(5)$ \\
\hline $\mathrm{C} 3 \mathrm{~A}$ & $4511(2)$ & $10060(2)$ & $-687.7(17)$ & $22.6(5)$ \\
\hline $\mathrm{C} 4 \mathrm{~A}$ & $3712(2)$ & $11166(2)$ & $-812.8(18)$ & $26.8(6)$ \\
\hline $\mathrm{C} 5 \mathrm{~A}$ & $2585(2)$ & $11495(2)$ & $-456.2(18)$ & $26.4(6)$ \\
\hline C6A & $2207(2)$ & $10750(2)$ & $36.9(17)$ & $24.7(6)$ \\
\hline C7A & $2999(2)$ & $9661(2)$ & $154.1(15)$ & $19.3(5)$ \\
\hline C8A & $2864(2)$ & $8691(2)$ & $653.4(15)$ & $20.5(5)$ \\
\hline C9A & $4257(2)$ & $6718(2)$ & 1041.1(16) & $21.7(5)$ \\
\hline C10A & $4610(2)$ & $6770.4(19)$ & $2040.2(16)$ & $21.2(5)$ \\
\hline C11A & $4365(2)$ & 7758.5(19) & $2448.3(15)$ & $22.3(5)$ \\
\hline C12A & $4145(2)$ & 8740.4(19) & $2842.6(16)$ & $23.8(5)$ \\
\hline C13A & $4951(2)$ & $9344(2)$ & $2823.3(17)$ & $22.2(5)$ \\
\hline C14A & $4425(3)$ & $10463(2)$ & $2288(2)$ & $41.5(7)$ \\
\hline C15A & $5073(3)$ & $9611(2)$ & $3853.8(18)$ & $38.7(7)$ \\
\hline C16A & $6104(3)$ & $8619(2)$ & $2353(2)$ & $40.4(7)$ \\
\hline C17A & $5175(2)$ & $5678(2)$ & $2536.2(16)$ & $20.0(5)$ \\
\hline C18A & $5258(2)$ & $4662(2)$ & $2161.9(17)$ & $22.0(5)$ \\
\hline C19A & $5776(2)$ & $3648(2)$ & $2651.9(17)$ & $22.0(5)$ \\
\hline C20A & $6214(2)$ & $3649(2)$ & $3520.1(16)$ & $19.4(5)$ \\
\hline $\mathrm{C} 21 \mathrm{~A}$ & $6133(2)$ & $4680(2)$ & $3910.6(16)$ & $18.1(5)$ \\
\hline $\mathrm{C} 22 \mathrm{~A}$ & $5634(2)$ & $5672(2)$ & $3418.1(16)$ & $18.0(5)$ \\
\hline $\mathrm{C} 23 \mathrm{~A}$ & $6350(2)$ & $5667(2)$ & $5250.4(17)$ & $24.2(5)$ \\
\hline C24A & $6844(2)$ & $1644(2)$ & $3667.0(18)$ & $26.7(6)$ \\
\hline O1D & $-132.4(15)$ & $10976.9(15)$ & $9306.8(12)$ & $25.8(4)$ \\
\hline O2D & $-515.3(16)$ & $8576.1(14)$ & $7131.1(12)$ & $25.9(4)$ \\
\hline O3D & 4307.9(16) & $3700.3(14)$ & $10110.1(12)$ & $25.0(4)$ \\
\hline O4D & $5272.3(14)$ & $4683.6(13)$ & 8938.4(11) & $21.7(4)$ \\
\hline N1D & $-183.9(17)$ & $9545.1(16)$ & $8361.2(13)$ & $19.4(4)$ \\
\hline C1D & $-362(2)$ & $10657(2)$ & $8578.1(16)$ & $20.4(5)$ \\
\hline $\mathrm{C} 2 \mathrm{D}$ & $-899(2)$ & $11338(2)$ & $7741.3(17)$ & $19.9(5)$ \\
\hline C3D & $-1235(2)$ & $12479(2)$ & $7572.4(17)$ & $23.7(5)$ \\
\hline C4D & $-1728(2)$ & $12885(2)$ & $6716.9(18)$ & $26.3(6)$ \\
\hline C5D & $-1865(2)$ & $12167(2)$ & $6054.6(17)$ & $24.7(6)$ \\
\hline \multirow[t]{2}{*}{ C6D } & $-1507(2)$ & $11016(2)$ & $6226.4(17)$ & $23.5(5)$ \\
\hline & & & $S-41$ & \\
\hline
\end{tabular}




\begin{tabular}{|c|c|c|c|c|}
\hline C7D & $-1023(2)$ & $10614(2)$ & $7081.8(16)$ & $18.7(5)$ \\
\hline C8D & $-566(2)$ & $9450(2)$ & $7470.6(16)$ & $19.7(5)$ \\
\hline C9D & $394(2)$ & $8568(2)$ & 8944.7(17) & $21.4(5)$ \\
\hline C10D & $1673(2)$ & 8042.1(19) & $8704.6(15)$ & $18.4(5)$ \\
\hline C11D & 2174.1(19) & $8598.9(17)$ & $8190.2(14)$ & $18.5(5)$ \\
\hline C12D & $2665(2)$ & $9156.5(18)$ & $7689.4(15)$ & $21.1(5)$ \\
\hline C13D & $2928(2)$ & $9079(2)$ & $6620.4(17)$ & $26.6(6)$ \\
\hline C14D & $2479(3)$ & $8292(3)$ & $6160.3(19)$ & $43.2(7)$ \\
\hline C15D & $2400(3)$ & $10275(3)$ & $6215(2)$ & $49.1(8)$ \\
\hline C16D & $4227(3)$ & $8616(2)$ & $6448(2)$ & $38.6(7)$ \\
\hline C17D & $2321(2)$ & $6889(2)$ & $9081.1(16)$ & $19.2(5)$ \\
\hline C18D & $1824(2)$ & $6366(2)$ & $9722.0(17)$ & $21.0(5)$ \\
\hline C19D & $2461(2)$ & $5297(2)$ & $10078.1(17)$ & $21.9(5)$ \\
\hline C20D & $3602(2)$ & $4737(2)$ & $9795.3(16)$ & $20.0(5)$ \\
\hline C21D & $4116(2)$ & $5267.4(19)$ & $9144.2(16)$ & $18.3(5)$ \\
\hline C22D & $3482(2)$ & $6319(2)$ & $8787.6(15)$ & $18.0(5)$ \\
\hline C23D & $5832(2)$ & $5235(2)$ & $8336.7(17)$ & $23.4(5)$ \\
\hline C24D & $3827(3)$ & $3192(2)$ & $10835.4(19)$ & $29.7(6)$ \\
\hline O1B & $8175.6(16)$ & $6209.2(15)$ & $3767.7(12)$ & $27.3(4)$ \\
\hline $\mathrm{O} 2 \mathrm{~B}$ & $11793.0(16)$ & $5069.4(16)$ & $2378.6(13)$ & $31.8(4)$ \\
\hline $\mathrm{O} 3 \mathrm{~B}$ & $7326.2(14)$ & $9115.8(13)$ & $-1263(1)$ & $19.3(4)$ \\
\hline $\mathrm{O} 4 \mathrm{~B}$ & $7209.5(15)$ & $11005.8(13)$ & $-545.3(11)$ & 21.3(4) \\
\hline $\mathrm{N} 1 \mathrm{~B}$ & $9914.1(18)$ & $5899.2(17)$ & $2971.5(13)$ & $21.3(4)$ \\
\hline C1B & $9144(2)$ & $5584(2)$ & $3520.3(16)$ & $21.3(5)$ \\
\hline $\mathrm{C} 2 \mathrm{~B}$ & $9772(2)$ & $4358(2)$ & $3731.7(16)$ & $21.5(5)$ \\
\hline $\mathrm{C} 3 \mathrm{~B}$ & $9413(2)$ & $3602(2)$ & $4213.4(17)$ & $25.9(6)$ \\
\hline $\mathrm{C} 4 \mathrm{~B}$ & $10222(3)$ & $2500(2)$ & 4299.6(18) & $29.7(6)$ \\
\hline $\mathrm{C} 5 \mathrm{~B}$ & $11336(3)$ & $2180(2)$ & 3928.2(19) & $30.9(6)$ \\
\hline C6B & $11692(2)$ & $2947(2)$ & $3439.6(18)$ & $26.7(6)$ \\
\hline C7B & $10883(2)$ & $4030(2)$ & $3344.1(16)$ & $22.4(5)$ \\
\hline C8B & $10978(2)$ & $5022(2)$ & $2832.5(16)$ & $22.2(5)$ \\
\hline C9B & $9590(2)$ & $6978(2)$ & $2491.9(17)$ & $23.5(5)$ \\
\hline $\mathrm{C} 10 \mathrm{~B}$ & $8917(2)$ & $7021(2)$ & $1628.3(16)$ & $20.8(5)$ \\
\hline $\mathrm{C} 11 \mathrm{~B}$ & $8588.5(19)$ & $6195.9(17)$ & $1475.4(14)$ & $18.4(4)$ \\
\hline C12B & $8210(2)$ & $5406.3(18)$ & $1394.2(15)$ & $20.0(5)$ \\
\hline C13B & $8755(2)$ & $4343.3(19)$ & $784.1(16)$ & $20.6(5)$ \\
\hline C14B & $7967(2)$ & $4437(2)$ & $-12.2(17)$ & $29.9(6)$ \\
\hline C15B & $8872(3)$ & $3309(2)$ & $1397.2(18)$ & $30.8(6)$ \\
\hline C16B & $9925(2)$ & $4218(2)$ & $352.8(19)$ & $33.2(6)$ \\
\hline
\end{tabular}




$\begin{array}{lllll}\text { C17B } & 8562(2) & 8059.1(19) & 1042.9(16) & 18.2(5) \\ \text { C18B } & 8130(2) & 8066(2) & 151.4(16) & 18.3(5) \\ \text { C19B } & 7712(2) & 9046(2) & -369.8(16) & 17.1(5) \\ \text { C20B } & 7679(2) & 10068.7(19) & 5.2(16) & 18.2(5) \\ \text { C21B } & 8130(2) & 10058(2) & 872.8(16) & 21.0(5) \\ \text { C22B } & 8578(2) & 9060(2) & 1385.0(16) & 21.2(5) \\ \text { C23B } & 7045(2) & 12072(2) & -133.6(19) & 26.8(6) \\ \text { C24B } & 7493(2) & 8071(2) & -1715.8(17) & 22.1(5)\end{array}$

Table A17. Anisotropic Displacement Parameters $\left(\AA^{2} \times 10^{3}\right)$. The Anisotropic displacement factor exponent takes the form: $-2 \pi^{2}\left[h^{2} a * 2 U_{11}+2 h k a * b * U_{12}+\ldots\right]$.

$\begin{array}{lllllll}\text { Atom } & \mathbf{U}_{11} & \mathbf{U}_{\mathbf{2 2}} & \mathbf{U} 3 \mathbf{3} & \mathbf{U}_{23} & \mathbf{U}_{\mathbf{1 3}} & \mathbf{U}_{\mathbf{1 2}} \\ \text { O1C } & 31.8(11) & 31.3(10) & 24.8(9) & -1.3(7) & -9.1(8) & -8.7(8) \\ \text { O2C } & 29.4(10) & 21.0(9) & 31.7(9) & 0.9(7) & -3.1(8) & -9.1(8) \\ \text { O3C } & 17.5(9) & 21.6(9) & 23.6(9) & 0.9(7) & -0.8(7) & -2.4(7) \\ \text { O4C } & 26.2(10) & 18.8(8) & 25.3(8) & 5.7(7) & -5.3(7) & -5.1(8) \\ \text { N1C } & 17.8(11) & 20.2(11) & 20.4(10) & 3.8(8) & -1.7(8) & -4.2(9) \\ \text { C1C } & 14.4(12) & 22.8(13) & 20.6(12) & -0.3(10) & 0.3(9) & -3.2(10) \\ \text { C2C } & 14.9(12) & 21.7(12) & 16.8(11) & 0.7(9) & -0.3(9) & -4.3(10) \\ \text { C3C } & 25.7(14) & 22.4(13) & 23.1(12) & -2.5(10) & -3.8(10) & -7.7(11) \\ \text { C4C } & 26.5(14) & 19.9(12) & 24.3(12) & 3.8(10) & -4.1(10) & -5.2(11) \\ \text { C5C } & 19.6(13) & 25.7(13) & 21.4(11) & 4.3(9) & -3.7(10) & -6.5(10) \\ \text { C6C } & 20.3(13) & 23.7(12) & 19.7(11) & -0.7(9) & -3.0(9) & -8.3(11) \\ \text { C7C } & 15.4(12) & 19.4(12) & 21.9(11) & 0.0(9) & 1.1(9) & -4.9(10) \\ \text { C8C } & 15.7(12) & 20.8(13) & 23.2(12) & 2.5(10) & 0.9(10) & -5.3(10) \\ \text { C9C } & 17.5(13) & 24.1(13) & 21.9(12) & 7.4(10) & -0.4(10) & -2.5(11) \\ \text { C10C } & 17.7(12) & 21.7(12) & 22.4(11) & 2.6(9) & 1.7(9) & -4.8(10) \\ \text { C11C } & 19.3(12) & 19.3(11) & 30.5(12) & 1.3(9) & -3.3(9) & -3.8(9) \\ \text { C12C } & 28.0(13) & 24.2(12) & 21.6(11) & 2.0(9) & -1.5(10) & -5.8(10) \\ \text { C13C } & 27.8(14) & 30.7(13) & 19.2(11) & 2.4(10) & 3.5(10) & -11.8(11) \\ \text { C14C } & 43.6(19) & 43.7(17) & 50.7(18) & 5.0(14) & 17.8(14) & -13.5(14) \\ \text { C15C } & 61(2) & 57.2(18) & 33.4(14) & 0.4(13) & 1.2(14) & -40.2(17) \\ \text { C16C } & 54(2) & 31.9(15) & 44.5(17) & 15.9(12) & -2.4(14) & -14.4(14) \\ \text { C17C } & 18.2(12) & 19.6(11) & 16.2(10) & 0.4(9) & -2.6(9) & -7.3(10) \\ \text { C18C } & 21.0(13) & 17.2(11) & 17.7(10) & 0.1(9) & -0.8(9) & -7.7(10) \\ \text { C19C } & 18.1(13) & 19.1(12) & 16.2(11) & -3.4(9) & -2.1(9) & -5.5(10) \\ \text { C20C } & 23.9(13) & 15.3(11) & 17.6(11) & 1.4(9) & -7.7(9) & -6.6(10) \\ \text { C21C } & 21.0(13) & 23.2(12) & 19.4(11) & 3.1(9) & -2.7(9) & -11.1(10)\end{array}$




\begin{tabular}{|c|c|c|c|c|c|c|}
\hline $\mathrm{C} 22 \mathrm{C}$ & 18.7(13) & $21.9(12)$ & 20.1(11) & $-0.1(9)$ & $-0.8(10)$ & $-7.2(10)$ \\
\hline $\mathrm{C} 23 \mathrm{C}$ & 19.7(13) & $27.5(14)$ & $25.3(13)$ & $-0.1(10)$ & $1.3(10)$ & $-6.8(11)$ \\
\hline $\mathrm{C} 24 \mathrm{C}$ & $31.7(15)$ & $22.3(13)$ & $35.7(14)$ & $12.4(11)$ & $-8.6(11)$ & $-13.9(12)$ \\
\hline O1A & $22.7(10)$ & $22.2(9)$ & $31.2(9)$ & $2.0(7)$ & $-0.3(7)$ & $-3.4(8)$ \\
\hline $\mathrm{O} 2 \mathrm{~A}$ & 24.1(10) & $32.6(10)$ & $23.8(8)$ & $4.8(7)$ & $-0.8(7)$ & $-11.4(8)$ \\
\hline $\mathrm{O} 3 \mathrm{~A}$ & $25.6(10)$ & 21.9(9) & $17.5(8)$ & $1.6(7)$ & $-5.6(7)$ & $-7.9(8)$ \\
\hline $\mathrm{O} 4 \mathrm{~A}$ & $28.2(10)$ & $16.4(8)$ & $26.9(9)$ & $3.5(7)$ & $-7.0(7)$ & $-5.7(7)$ \\
\hline N1A & $21.8(11)$ & $17.8(10)$ & 19.1(9) & $2.8(8)$ & $-4.6(8)$ & $-6.2(9)$ \\
\hline $\mathrm{C} 1 \mathrm{~A}$ & 19.7(13) & 21.1(12) & $15.7(11)$ & $0.1(9)$ & $-4.5(9)$ & $-5.9(11)$ \\
\hline $\mathrm{C} 2 \mathrm{~A}$ & 20.3(13) & $21.8(12)$ & $14.4(10)$ & $-0.7(9)$ & $-3.7(9)$ & $-6.8(10)$ \\
\hline C3A & $22.8(14)$ & $22.7(12)$ & $20.4(11)$ & $1.9(9)$ & $-1.2(10)$ & $-7.2(11)$ \\
\hline $\mathrm{C} 4 \mathrm{~A}$ & 28.3(14) & $22.5(13)$ & $28.6(13)$ & $4.2(10)$ & $0.2(11)$ & $-9.4(11)$ \\
\hline C5A & 26.4(14) & $18.2(12)$ & $28.5(13)$ & $3.3(10)$ & $-2.3(11)$ & $-2.6(11)$ \\
\hline C6A & $19.6(13)$ & $27.8(14)$ & $22.2(12)$ & $1.4(10)$ & $-2.2(10)$ & $-4.8(11)$ \\
\hline C7A & $20.0(13)$ & $23.2(12)$ & $13.9(10)$ & $-0.6(9)$ & $-4.2(9)$ & $-7.3(10)$ \\
\hline C8A & $21.5(14)$ & $25.6(13)$ & $14.2(11)$ & $0.9(9)$ & $-3.3(10)$ & $-8.8(11)$ \\
\hline C9A & 27.9(14) & $17.1(12)$ & $20.6(12)$ & $3.7(9)$ & $-6.1(10)$ & $-8.9(11)$ \\
\hline C10A & $29.4(14)$ & $17.4(11)$ & $20.7(11)$ & $4.3(9)$ & $-6.3(10)$ & $-12.8(10)$ \\
\hline C11A & $22.8(12)$ & $26.8(12)$ & $20.2(10)$ & $5.5(9)$ & $-5.6(9)$ & $-12.3(10)$ \\
\hline $\mathrm{C} 12 \mathrm{~A}$ & 23.6(12) & $23.5(12)$ & $22.0(11)$ & $1.2(9)$ & $-1.8(9)$ & $-7(1)$ \\
\hline $\mathrm{C} 13 \mathrm{~A}$ & $27.5(14)$ & $19.7(12)$ & $20.4(11)$ & $0.1(9)$ & $-1.5(10)$ & $-10.5(11)$ \\
\hline C14A & $66(2)$ & $24.5(13)$ & $37.7(15)$ & $6.2(11)$ & $-10.4(14)$ & $-20.2(14)$ \\
\hline $\mathrm{C} 15 \mathrm{~A}$ & $56.6(19)$ & $47.0(16)$ & 27.1(13) & $1.5(11)$ & $-8.0(12)$ & $-35.4(15)$ \\
\hline C16A & $35.5(16)$ & $42.7(16)$ & $45.3(16)$ & $-5.3(13)$ & $7.3(13)$ & $-19.4(13)$ \\
\hline C17A & $22.0(13)$ & $22.9(12)$ & $17.5(11)$ & $3.1(9)$ & $-2.2(9)$ & $-11.4(10)$ \\
\hline $\mathrm{C} 18 \mathrm{~A}$ & $28.0(14)$ & $21.0(12)$ & $19.7(11)$ & $4.0(9)$ & $-6.5(10)$ & $-11.8(11)$ \\
\hline C19A & $24.4(14)$ & $18.8(12)$ & $23.6(12)$ & $-1.5(9)$ & $-1.9(10)$ & $-9.4(11)$ \\
\hline $\mathrm{C} 20 \mathrm{~A}$ & $15.8(12)$ & $18.2(12)$ & $22.4(12)$ & $4.9(9)$ & $-0.2(10)$ & $-5.1(10)$ \\
\hline $\mathrm{C} 21 \mathrm{~A}$ & $13.7(12)$ & $22.4(12)$ & $17.0(11)$ & $-0.1(9)$ & $-0.3(9)$ & $-6(1)$ \\
\hline $\mathrm{C} 22 \mathrm{~A}$ & 20.1(12) & $17.8(11)$ & $17.9(11)$ & $1.2(9)$ & $-1.3(9)$ & $-9.6(10)$ \\
\hline $\mathrm{C} 23 \mathrm{~A}$ & $29.7(15)$ & $23.9(13)$ & $20.6(12)$ & $-0.7(10)$ & $-5.4(11)$ & $-11.6(11)$ \\
\hline $\mathrm{C} 24 \mathrm{~A}$ & $30.4(15)$ & $14.9(12)$ & $30.9(13)$ & $-0.5(10)$ & $-3.3(11)$ & $-4.5(11)$ \\
\hline O1D & $24.9(10)$ & $29.4(10)$ & $22.3(8)$ & $1.1(7)$ & $-7.2(7)$ & $-9.1(8)$ \\
\hline $\mathrm{O} 2 \mathrm{D}$ & $30(1)$ & $19.3(9)$ & $27.5(9)$ & $-0.5(7)$ & $-2.3(7)$ & $-8.6(8)$ \\
\hline O3D & 26.7(10) & $16.3(8)$ & $27.9(9)$ & $6.0(7)$ & $-3.2(7)$ & $-4.2(7)$ \\
\hline $\mathrm{O} 4 \mathrm{D}$ & $16.7(9)$ & $19.8(9)$ & $23.2(8)$ & $-0.5(7)$ & $0.2(7)$ & $-1.7(7)$ \\
\hline N1D & $15.5(11)$ & $19.3(10)$ & $20.2(10)$ & $4.2(8)$ & $-2.5(8)$ & $-3.3(8)$ \\
\hline C1D & $15.0(12)$ & $24.1(13)$ & $20.3(12)$ & $2.1(10)$ & $-0.7(10)$ & $-5.9(10)$ \\
\hline $\mathrm{C} 2 \mathrm{D}$ & $14.7(12)$ & $22.3(12)$ & 21.1(11) & $1.2(9)$ & $0.2(9)$ & $-5.9(10)$ \\
\hline
\end{tabular}




\begin{tabular}{|c|c|c|c|c|c|c|}
\hline C3D & $25.7(14)$ & $20.1(12)$ & $22.8(12)$ & $0(1)$ & $-0.6(10)$ & $-6.6(11)$ \\
\hline C4D & $25.9(14)$ & $20.4(12)$ & $28.9(13)$ & $6.1(10)$ & $-0.7(11)$ & $-5.7(11)$ \\
\hline C5D & $23.2(14)$ & $28.3(13)$ & $19.8(11)$ & $6.3(10)$ & $-1.6(10)$ & $-7.6(11)$ \\
\hline C6D & $22.6(14)$ & 28.1(13) & $20.5(12)$ & $2.7(10)$ & $-2.4(10)$ & $-10.9(11)$ \\
\hline C7D & $14.8(12)$ & $21.0(12)$ & $19.4(11)$ & $2.1(9)$ & $-0.2(9)$ & $-6.4(10)$ \\
\hline C8D & $15.2(12)$ & $23.7(13)$ & $19.6(11)$ & $-0.4(10)$ & $1.1(9)$ & $-7.3(10)$ \\
\hline C9D & $16.2(12)$ & $20.4(12)$ & $23.8(12)$ & $6.5(9)$ & $-1.1(10)$ & $-3.7(10)$ \\
\hline C10D & $18.9(12)$ & $19.0(11)$ & $14.6(10)$ & $2.3(8)$ & $0.3(9)$ & $-4.9(10)$ \\
\hline C11D & $16.7(11)$ & $16.5(10)$ & $17.2(10)$ & $-1.2(8)$ & $-3.5(8)$ & $-0.6(9)$ \\
\hline C12D & $22.4(12)$ & $19.6(11)$ & $21.4(11)$ & $1.3(8)$ & $-1.2(9)$ & $-8.6(9)$ \\
\hline C13D & $32.3(15)$ & $28.1(13)$ & $20.0(12)$ & $4.4(10)$ & $1.3(10)$ & $-13.4(12)$ \\
\hline C14D & $52.5(19)$ & $64.1(19)$ & $23.4(13)$ & $-8.5(12)$ & $3.1(12)$ & $-34.5(16)$ \\
\hline C15D & $59(2)$ & $42.7(17)$ & $34.3(15)$ & $17.2(13)$ & $4.4(14)$ & $-10.3(16)$ \\
\hline C16D & $38.5(17)$ & $42.0(15)$ & $33.2(14)$ & $1.8(12)$ & $9.5(12)$ & $-15.6(13)$ \\
\hline C17D & $19.4(13)$ & $18.2(12)$ & $18.4(11)$ & $-0.2(9)$ & $-1.9(9)$ & $-5.6(10)$ \\
\hline C18D & $16.8(13)$ & $22.2(13)$ & $22.7(12)$ & $3.1(9)$ & $-1(1)$ & $-6.5(10)$ \\
\hline C19D & $25.0(13)$ & $22.0(12)$ & $21.3(11)$ & $4.6(9)$ & $-2.7(10)$ & $-12.2(10)$ \\
\hline C20D & $23.2(13)$ & $17.5(12)$ & $18.5(11)$ & $0.0(9)$ & $-4.2(10)$ & $-6.8(10)$ \\
\hline C21D & $16.2(12)$ & $17.8(12)$ & $17.9(11)$ & $-4.8(9)$ & $-2.5(9)$ & $-3.1(10)$ \\
\hline C22D & $19.3(13)$ & $21.5(12)$ & $13.5(10)$ & $0.5(9)$ & $-1.8(9)$ & $-8.3(10)$ \\
\hline C23D & $16.2(13)$ & $25.4(13)$ & $25.1(12)$ & $-1.2(10)$ & $0.8(10)$ & $-4.8(10)$ \\
\hline C24D & $32.9(16)$ & $21.4(13)$ & $35.1(14)$ & $9.5(11)$ & $-7.0(12)$ & $-10.8(12)$ \\
\hline O1B & $24.9(10)$ & 24.1(9) & $27.0(9)$ & $0.8(7)$ & $1.5(8)$ & $-4.0(8)$ \\
\hline $\mathrm{O} 2 \mathrm{~B}$ & $29.0(11)$ & $38.1(11)$ & $29.9(9)$ & $3.9(8)$ & $1.5(8)$ & $-15.8(9)$ \\
\hline $\mathrm{O} 3 \mathrm{~B}$ & $21.9(9)$ & $17.3(8)$ & $17.2(8)$ & $-1.2(6)$ & $-5.1(7)$ & $-5.6(7)$ \\
\hline O4B & 24.4(9) & $14.4(8)$ & $23.4(8)$ & $1.8(6)$ & $-7.2(7)$ & $-5.0(7)$ \\
\hline N1B & $23.5(11)$ & $24.4(11)$ & 17.1(9) & $3.1(8)$ & $-4.0(8)$ & $-10.5(9)$ \\
\hline $\mathrm{C} 1 \mathrm{~B}$ & $27.6(15)$ & $22.2(12)$ & $15.8(11)$ & $0.3(9)$ & $-4.1(10)$ & $-11.1(11)$ \\
\hline $\mathrm{C} 2 \mathrm{~B}$ & $25.4(14)$ & $22.3(12)$ & $15.8(11)$ & $0.0(9)$ & $-4.3(10)$ & $-8.2(11)$ \\
\hline $\mathrm{C} 3 \mathrm{~B}$ & $25.8(14)$ & $28.2(14)$ & $22.2(12)$ & $3.6(10)$ & $-1.2(10)$ & $-9.4(11)$ \\
\hline C4B & $37.3(16)$ & $25.6(14)$ & $24.2(12)$ & $6.5(10)$ & $-2.3(11)$ & $-10.5(12)$ \\
\hline $\mathrm{C} 5 \mathrm{~B}$ & $32.6(16)$ & $23.7(14)$ & $29.4(13)$ & $4.9(10)$ & $-7.8(11)$ & $-3.1(12)$ \\
\hline C6B & $23.7(14)$ & $28.3(14)$ & $24.4(12)$ & $0.2(10)$ & $-3.6(10)$ & $-5.9(11)$ \\
\hline $\mathrm{C} 7 \mathrm{~B}$ & $24.5(14)$ & $25.8(13)$ & $16.7(11)$ & $1.8(9)$ & $-6.2(10)$ & $-9.2(11)$ \\
\hline $\mathrm{C} 8 \mathrm{~B}$ & $23.8(14)$ & 28.7(13) & $15.8(11)$ & $0.5(9)$ & $-5.2(10)$ & $-11.7(11)$ \\
\hline C9B & $31.9(15)$ & $22.3(13)$ & $20.0(11)$ & $2.4(10)$ & $-6.3(10)$ & $-14.0(11)$ \\
\hline $\mathrm{C} 10 \mathrm{~B}$ & $24.0(13)$ & $20.3(11)$ & $19.2(11)$ & $0.4(9)$ & $-4.0(9)$ & $-9.5(10)$ \\
\hline C11B & $18.6(11)$ & $18.3(10)$ & $14.8(9)$ & $2.6(8)$ & $-1.3(8)$ & $-3.8(9)$ \\
\hline C12B & $22.1(12)$ & $20.1(11)$ & $18.5(10)$ & $0.8(8)$ & $-2.0(9)$ & $-9.1(9)$ \\
\hline
\end{tabular}




$\begin{array}{lllllll}\text { C13B } & 25.2(13) & 18.0(12) & 20.7(11) & -1.8(9) & -1.9(10) & -10.8(10) \\ \text { C14B } & 39.5(16) & 24.7(12) & 27.8(12) & -0.4(9) & -11.1(11) & -13.9(11) \\ \text { C15B } & 42.8(16) & 19.5(12) & 30.7(13) & 3.5(10) & -7.5(12) & -12.3(12) \\ \text { C16B } & 28.8(14) & 31.2(13) & 37.9(14) & -8.0(11) & 7.9(11) & -11.2(11) \\ \text { C17B } & 17.1(12) & 17.6(12) & 20.1(11) & 1.1(9) & -2.4(9) & -7(1) \\ \text { C18B } & 17.1(12) & 16.9(11) & 21.2(11) & -3.1(9) & 0.3(9) & -7.2(9) \\ \text { C19B } & 13.4(12) & 21.3(12) & 16.9(11) & -0.4(9) & 0.6(9) & -7.3(10) \\ \text { C20B } & 15.5(12) & 16.7(12) & 20.6(11) & 1.2(9) & -0.5(9) & -4.6(10) \\ \text { C21B } & 24.6(14) & 18.8(12) & 21.7(12) & -1.9(9) & -2.9(10) & -10.6(11) \\ \text { C22B } & 24.0(14) & 23.5(13) & 18.7(12) & -1.2(10) & -3.8(10) & -11.5(11) \\ \text { C23B } & 27.4(15) & 15.1(12) & 33.1(14) & -0.7(10) & -6.0(11) & -2.7(11) \\ \text { C24B } & 28.2(14) & 20.5(12) & 19.2(11) & -1.9(9) & -2.3(10) & -11.1(11)\end{array}$

Table A18. Bond Lengths.

\begin{tabular}{|c|c|c|c|c|c|}
\hline Atom & Atom & Length/Å & Atom & Atom & Length/Å \\
\hline $\mathrm{O} 1 \mathrm{C}$ & $\mathrm{C} 1 \mathrm{C}$ & $1.215(3)$ & O1D & C1D & $1.210(3)$ \\
\hline $\mathrm{O} 2 \mathrm{C}$ & $\mathrm{C} 8 \mathrm{C}$ & $1.211(3)$ & $\mathrm{O} 2 \mathrm{D}$ & C8D & $1.206(3)$ \\
\hline $\mathrm{O} 3 \mathrm{C}$ & C19C & $1.362(3)$ & O3D & C20D & $1.366(3)$ \\
\hline $\mathrm{O} 3 \mathrm{C}$ & $\mathrm{C} 23 \mathrm{C}$ & $1.434(3)$ & O3D & C24D & $1.436(3)$ \\
\hline $\mathrm{O} 4 \mathrm{C}$ & C20C & $1.373(3)$ & O4D & C21D & $1.379(3)$ \\
\hline $\mathrm{O} 4 \mathrm{C}$ & $\mathrm{C} 24 \mathrm{C}$ & $1.428(3)$ & O4D & C23D & $1.427(3)$ \\
\hline $\mathrm{N} 1 \mathrm{C}$ & $\mathrm{C} 1 \mathrm{C}$ & $1.386(3)$ & N1D & C1D & $1.393(3)$ \\
\hline $\mathrm{N} 1 \mathrm{C}$ & $\mathrm{C} 8 \mathrm{C}$ & $1.397(3)$ & N1D & C8D & $1.402(3)$ \\
\hline $\mathrm{N} 1 \mathrm{C}$ & $\mathrm{C} 9 \mathrm{C}$ & $1.455(3)$ & N1D & C9D & $1.453(3)$ \\
\hline $\mathrm{C} 1 \mathrm{C}$ & $\mathrm{C} 2 \mathrm{C}$ & $1.488(3)$ & C1D & $\mathrm{C} 2 \mathrm{D}$ & $1.491(3)$ \\
\hline $\mathrm{C} 2 \mathrm{C}$ & $\mathrm{C} 3 \mathrm{C}$ & $1.386(3)$ & $\mathrm{C} 2 \mathrm{D}$ & C3D & $1.377(3)$ \\
\hline $\mathrm{C} 2 \mathrm{C}$ & $\mathrm{C} 7 \mathrm{C}$ & $1.382(3)$ & $\mathrm{C} 2 \mathrm{D}$ & C7D & $1.387(3)$ \\
\hline $\mathrm{C} 3 \mathrm{C}$ & $\mathrm{C} 4 \mathrm{C}$ & $1.391(3)$ & $\mathrm{C} 3 \mathrm{D}$ & C4D & $1.394(4)$ \\
\hline $\mathrm{C} 4 \mathrm{C}$ & $\mathrm{C} 5 \mathrm{C}$ & $1.387(4)$ & C4D & C5D & $1.393(4)$ \\
\hline $\mathrm{C} 5 \mathrm{C}$ & C6C & $1.400(4)$ & C5D & C6D & $1.387(4)$ \\
\hline C6C & $\mathrm{C} 7 \mathrm{C}$ & $1.382(3)$ & C6D & C7D & $1.388(3)$ \\
\hline $\mathrm{C} 7 \mathrm{C}$ & $\mathrm{C} 8 \mathrm{C}$ & $1.497(3)$ & C7D & C8D & $1.485(3)$ \\
\hline C9C & $\mathrm{C} 10 \mathrm{C}$ & $1.521(3)$ & C9D & C10D & $1.517(3)$ \\
\hline $\mathrm{C} 10 \mathrm{C}$ & $\mathrm{C} 11 \mathrm{C}$ & $1.308(3)$ & C10D & C11D & $1.317(3)$ \\
\hline $\mathrm{C} 10 \mathrm{C}$ & $\mathrm{C} 17 \mathrm{C}$ & $1.488(3)$ & C10D & C17D & $1.492(3)$ \\
\hline $\mathrm{C} 11 \mathrm{C}$ & $\mathrm{C} 12 \mathrm{C}$ & $1.306(3)$ & C11D & C12D & $1.299(3)$ \\
\hline $\mathrm{C} 12 \mathrm{C}$ & $\mathrm{C} 13 \mathrm{C}$ & $1.515(4)$ & C12D & C13D & $1.518(3)$ \\
\hline $\mathrm{C} 13 \mathrm{C}$ & $\mathrm{C} 14 \mathrm{C}$ & $1.525(4)$ & C13D & C14D & $1.520(4)$ \\
\hline $\mathrm{C} 13 \mathrm{C}$ & $\mathrm{C} 15 \mathrm{C}$ & $1.518(4)$ & $\begin{array}{l}\text { C13D } \\
S-46\end{array}$ & C15D & $1.532(4)$ \\
\hline
\end{tabular}




\begin{tabular}{|c|c|c|c|c|c|}
\hline $\mathrm{C} 13 \mathrm{C}$ & $\mathrm{C} 16 \mathrm{C}$ & $1.531(4)$ & C13D & C16D & $1.530(4)$ \\
\hline $\mathrm{C} 17 \mathrm{C}$ & $\mathrm{C} 18 \mathrm{C}$ & $1.404(3)$ & C17D & C18D & $1.383(3)$ \\
\hline $\mathrm{C} 17 \mathrm{C}$ & $\mathrm{C} 22 \mathrm{C}$ & $1.388(3)$ & C17D & C22D & $1.406(3)$ \\
\hline C18C & C19C & $1.382(3)$ & C18D & C19D & $1.395(3)$ \\
\hline C19C & $\mathrm{C} 20 \mathrm{C}$ & $1.408(3)$ & C19D & C20D & $1.380(4)$ \\
\hline $\mathrm{C} 20 \mathrm{C}$ & $\mathrm{C} 21 \mathrm{C}$ & $1.380(4)$ & C20D & C21D & $1.411(4)$ \\
\hline $\mathrm{C} 21 \mathrm{C}$ & $\mathrm{C} 22 \mathrm{C}$ & $1.396(3)$ & C21D & C22D & $1.378(3)$ \\
\hline O1A & $\mathrm{C} 1 \mathrm{~A}$ & $1.214(3)$ & O1B & $\mathrm{C} 1 \mathrm{~B}$ & $1.210(3)$ \\
\hline $\mathrm{O} 2 \mathrm{~A}$ & $\mathrm{C} 8 \mathrm{~A}$ & $1.207(3)$ & $\mathrm{O} 2 \mathrm{~B}$ & $\mathrm{C} 8 \mathrm{~B}$ & $1.208(3)$ \\
\hline O3A & $\mathrm{C} 21 \mathrm{~A}$ & $1.369(3)$ & O3B & C19B & $1.372(3)$ \\
\hline O3A & $\mathrm{C} 23 \mathrm{~A}$ & 1.441(3) & O3B & $\mathrm{C} 24 \mathrm{~B}$ & $1.430(3)$ \\
\hline $\mathrm{O} 4 \mathrm{~A}$ & $\mathrm{C} 20 \mathrm{~A}$ & $1.366(3)$ & O4B & $\mathrm{C} 20 \mathrm{~B}$ & $1.368(3)$ \\
\hline $\mathrm{O} 4 \mathrm{~A}$ & $\mathrm{C} 24 \mathrm{~A}$ & $1.435(3)$ & O4B & $\mathrm{C} 23 \mathrm{~B}$ & $1.430(3)$ \\
\hline N1A & $\mathrm{C} 1 \mathrm{~A}$ & $1.389(3)$ & N1B & $\mathrm{C} 1 \mathrm{~B}$ & $1.391(3)$ \\
\hline N1A & $\mathrm{C} 8 \mathrm{~A}$ & $1.406(3)$ & N1B & $\mathrm{C} 8 \mathrm{~B}$ & $1.384(3)$ \\
\hline N1A & C9A & $1.452(3)$ & N1B & C9B & $1.450(3)$ \\
\hline $\mathrm{C} 1 \mathrm{~A}$ & $\mathrm{C} 2 \mathrm{~A}$ & $1.490(3)$ & $\mathrm{C} 1 \mathrm{~B}$ & $\mathrm{C} 2 \mathrm{~B}$ & $1.493(3)$ \\
\hline $\mathrm{C} 2 \mathrm{~A}$ & $\mathrm{C} 3 \mathrm{~A}$ & $1.386(4)$ & $\mathrm{C} 2 \mathrm{~B}$ & C3B & $1.380(4)$ \\
\hline $\mathrm{C} 2 \mathrm{~A}$ & C7A & $1.386(4)$ & $\mathrm{C} 2 \mathrm{~B}$ & $\mathrm{C} 7 \mathrm{~B}$ & $1.386(4)$ \\
\hline C3A & $\mathrm{C} 4 \mathrm{~A}$ & $1.400(4)$ & C3B & C4B & $1.393(4)$ \\
\hline $\mathrm{C} 4 \mathrm{~A}$ & C5A & $1.391(4)$ & C4B & C5B & $1.384(4)$ \\
\hline $\mathrm{C} 5 \mathrm{~A}$ & C6A & $1.390(4)$ & $\mathrm{C} 5 \mathrm{~B}$ & $\mathrm{C} 6 \mathrm{~B}$ & $1.395(4)$ \\
\hline C6A & $\mathrm{C} 7 \mathrm{~A}$ & $1.380(3)$ & C6B & $\mathrm{C} 7 \mathrm{~B}$ & $1.377(4)$ \\
\hline C7A & $\mathrm{C} 8 \mathrm{~A}$ & $1.484(3)$ & C7B & $\mathrm{C} 8 \mathrm{~B}$ & $1.497(3)$ \\
\hline C9A & $\mathrm{C} 10 \mathrm{~A}$ & $1.518(3)$ & C9B & $\mathrm{C} 10 \mathrm{~B}$ & $1.527(3)$ \\
\hline C10A & C11A & $1.315(3)$ & C10B & C11B & $1.313(3)$ \\
\hline C10A & $\mathrm{C} 17 \mathrm{~A}$ & $1.487(3)$ & $\mathrm{C} 10 \mathrm{~B}$ & $\mathrm{C} 17 \mathrm{~B}$ & $1.484(3)$ \\
\hline C11A & $\mathrm{C} 12 \mathrm{~A}$ & $1.306(3)$ & C11B & $\mathrm{C} 12 \mathrm{~B}$ & $1.298(3)$ \\
\hline C12A & $\mathrm{C} 13 \mathrm{~A}$ & $1.516(4)$ & C12B & $\mathrm{C} 13 \mathrm{~B}$ & $1.516(3)$ \\
\hline $\mathrm{C} 13 \mathrm{~A}$ & C14A & $1.534(3)$ & C13B & C14B & $1.531(3)$ \\
\hline C13A & $\mathrm{C} 15 \mathrm{~A}$ & $1.531(3)$ & C13B & $\mathrm{C} 15 \mathrm{~B}$ & $1.535(3)$ \\
\hline $\mathrm{C} 13 \mathrm{~A}$ & C16A & $1.511(4)$ & C13B & C16B & $1.524(4)$ \\
\hline C17A & $\mathrm{C} 18 \mathrm{~A}$ & $1.381(3)$ & C17B & C18B & $1.405(3)$ \\
\hline C17A & $\mathrm{C} 22 \mathrm{~A}$ & $1.409(3)$ & C17B & $\mathrm{C} 22 \mathrm{~B}$ & $1.391(3)$ \\
\hline $\mathrm{C} 18 \mathrm{~A}$ & C19A & $1.400(3)$ & C18B & C19B & $1.378(3)$ \\
\hline C19A & $\mathrm{C} 20 \mathrm{~A}$ & $1.380(3)$ & C19B & $\mathrm{C} 20 \mathrm{~B}$ & $1.411(3)$ \\
\hline $\mathrm{C} 20 \mathrm{~A}$ & $\mathrm{C} 21 \mathrm{~A}$ & $1.407(3)$ & C20B & $\mathrm{C} 21 \mathrm{~B}$ & $1.384(3)$ \\
\hline $\mathrm{C} 21 \mathrm{~A}$ & $\mathrm{C} 22 \mathrm{~A}$ & $1.379(3)$ & C21B & C22B & $1.391(3)$ \\
\hline
\end{tabular}


Table A19. Bond Angles.

\begin{tabular}{|c|c|c|c|}
\hline Atom & Atom & Atom & Angle $/^{\circ}$ \\
\hline C19C & $\mathrm{O} 3 \mathrm{C}$ & $\mathrm{C} 23 \mathrm{C}$ & $116.61(19)$ \\
\hline C20C & $\mathrm{O} 4 \mathrm{C}$ & $\mathrm{C} 24 \mathrm{C}$ & $116.8(2)$ \\
\hline $\mathrm{C} 1 \mathrm{C}$ & $\mathrm{N} 1 \mathrm{C}$ & $\mathrm{C} 8 \mathrm{C}$ & $112.3(2)$ \\
\hline $\mathrm{C} 1 \mathrm{C}$ & $\mathrm{N} 1 \mathrm{C}$ & C9C & $124.5(2)$ \\
\hline $\mathrm{C} 8 \mathrm{C}$ & $\mathrm{N} 1 \mathrm{C}$ & C9C & $123.1(2)$ \\
\hline $\mathrm{O} 1 \mathrm{C}$ & $\mathrm{C} 1 \mathrm{C}$ & $\mathrm{N} 1 \mathrm{C}$ & $125.4(2)$ \\
\hline $\mathrm{O} 1 \mathrm{C}$ & $\mathrm{C} 1 \mathrm{C}$ & $\mathrm{C} 2 \mathrm{C}$ & $128.6(2)$ \\
\hline $\mathrm{N} 1 \mathrm{C}$ & $\mathrm{C} 1 \mathrm{C}$ & $\mathrm{C} 2 \mathrm{C}$ & $105.9(2)$ \\
\hline $\mathrm{C} 3 \mathrm{C}$ & $\mathrm{C} 2 \mathrm{C}$ & $\mathrm{C} 1 \mathrm{C}$ & $130.2(2)$ \\
\hline C7C & $\mathrm{C} 2 \mathrm{C}$ & $\mathrm{C} 1 \mathrm{C}$ & $108.3(2)$ \\
\hline $\mathrm{C} 7 \mathrm{C}$ & $\mathrm{C} 2 \mathrm{C}$ & $\mathrm{C} 3 \mathrm{C}$ & $121.4(2)$ \\
\hline $\mathrm{C} 2 \mathrm{C}$ & $\mathrm{C} 3 \mathrm{C}$ & $\mathrm{C} 4 \mathrm{C}$ & $117.1(2)$ \\
\hline C5C & $\mathrm{C} 4 \mathrm{C}$ & $\mathrm{C} 3 \mathrm{C}$ & $121.5(2)$ \\
\hline $\mathrm{C} 4 \mathrm{C}$ & $\mathrm{C} 5 \mathrm{C}$ & C6C & $121.0(2)$ \\
\hline C7C & C6C & C5C & $116.9(2)$ \\
\hline $\mathrm{C} 2 \mathrm{C}$ & $\mathrm{C} 7 \mathrm{C}$ & C6C & $122.0(2)$ \\
\hline $\mathrm{C} 2 \mathrm{C}$ & C7C & $\mathrm{C} 8 \mathrm{C}$ & $108.0(2)$ \\
\hline C6C & C7C & $\mathrm{C} 8 \mathrm{C}$ & $130.0(2)$ \\
\hline $\mathrm{O} 2 \mathrm{C}$ & $\mathrm{C} 8 \mathrm{C}$ & $\mathrm{N} 1 \mathrm{C}$ & $125.3(2)$ \\
\hline $\mathrm{O} 2 \mathrm{C}$ & $\mathrm{C} 8 \mathrm{C}$ & C7C & $129.2(2)$ \\
\hline N1C & $\mathrm{C} 8 \mathrm{C}$ & C7C & $105.4(2)$ \\
\hline $\mathrm{N} 1 \mathrm{C}$ & $\mathrm{C} 9 \mathrm{C}$ & $\mathrm{C} 10 \mathrm{C}$ & $112.3(2)$ \\
\hline $\mathrm{C} 11 \mathrm{C}$ & $\mathrm{C} 10 \mathrm{C}$ & C9C & $119.8(2)$ \\
\hline $\mathrm{C} 11 \mathrm{C}$ & $\mathrm{C} 10 \mathrm{C}$ & $\mathrm{C} 17 \mathrm{C}$ & $123.0(2)$ \\
\hline $\mathrm{C} 17 \mathrm{C}$ & $\mathrm{C} 10 \mathrm{C}$ & C9C & $116.8(2)$ \\
\hline $\mathrm{C} 12 \mathrm{C}$ & $\mathrm{C} 11 \mathrm{C}$ & $\mathrm{C} 10 \mathrm{C}$ & $175.7(3)$ \\
\hline $\mathrm{C} 11 \mathrm{C}$ & $\mathrm{C} 12 \mathrm{C}$ & $\mathrm{C} 13 \mathrm{C}$ & $126.4(2)$ \\
\hline $\mathrm{C} 12 \mathrm{C}$ & $\mathrm{C} 13 \mathrm{C}$ & $\mathrm{C} 14 \mathrm{C}$ & $108.5(2)$ \\
\hline $\mathrm{C} 12 \mathrm{C}$ & $\mathrm{C} 13 \mathrm{C}$ & $\mathrm{C} 15 \mathrm{C}$ & $111.1(2)$ \\
\hline $\mathrm{C} 12 \mathrm{C}$ & $\mathrm{C} 13 \mathrm{C}$ & $\mathrm{C} 16 \mathrm{C}$ & $108.5(2)$ \\
\hline $\mathrm{C} 14 \mathrm{C}$ & $\mathrm{C} 13 \mathrm{C}$ & $\mathrm{C} 16 \mathrm{C}$ & $109.3(2)$ \\
\hline $\mathrm{C} 15 \mathrm{C}$ & $\mathrm{C} 13 \mathrm{C}$ & $\mathrm{C} 14 \mathrm{C}$ & $110.0(3)$ \\
\hline $\mathrm{C} 15 \mathrm{C}$ & $\mathrm{C} 13 \mathrm{C}$ & $\mathrm{C} 16 \mathrm{C}$ & $109.3(2)$ \\
\hline C18C & $\mathrm{C} 17 \mathrm{C}$ & $\mathrm{C} 10 \mathrm{C}$ & $119.5(2)$ \\
\hline $\mathrm{C} 22 \mathrm{C}$ & $\mathrm{C} 17 \mathrm{C}$ & $\mathrm{C} 10 \mathrm{C}$ & $121.8(2)$ \\
\hline $\mathrm{C} 22 \mathrm{C}$ & $\mathrm{C} 17 \mathrm{C}$ & $\mathrm{C} 18 \mathrm{C}$ & $118.7(2)$ \\
\hline
\end{tabular}

\begin{tabular}{|c|c|c|c|}
\hline Atom & Atom & Atom & Angle $/^{\circ}$ \\
\hline C20D & O3D & C24D & $116.5(2)$ \\
\hline $21 \mathrm{D}$ & O4D & C23D & $116.70(18)$ \\
\hline C1D & N1D & C8D & $112.21(19)$ \\
\hline C1D & N1D & C9D & $125.2(2)$ \\
\hline $8 D$ & N1D & C9D & $122.5(2)$ \\
\hline O1D & C1D & N1D & $125.7(2)$ \\
\hline O1D & C1D & C2D & $128.7(2)$ \\
\hline N1D & C1D & C2D & $105.58(19)$ \\
\hline C3D & $\mathrm{C} 2 \mathrm{D}$ & C1D & $130.0(2)$ \\
\hline C3D & C2D & C7D & $121.6(2)$ \\
\hline C7D & C2D & C1D & $108.3(2)$ \\
\hline $\mathrm{C} 2 \mathrm{D}$ & C3D & C4D & $117.2(2)$ \\
\hline C5D & C4D & C3D & $121.5(2)$ \\
\hline C6D & C5D & C4D & $120.7(2)$ \\
\hline C5D & C6D & C7D & $117.7(2)$ \\
\hline $\mathrm{C} 2 \mathrm{D}$ & C7D & C6D & $121.2(2)$ \\
\hline $\mathrm{C} 2 \mathrm{D}$ & C7D & C8D & $108.21(19)$ \\
\hline C6D & C7D & C8D & $130.6(2)$ \\
\hline $\mathrm{O} 2 \mathrm{D}$ & C8D & N1D & $124.6(2)$ \\
\hline $\mathrm{O} 2 \mathrm{D}$ & C8D & C7D & $129.8(2)$ \\
\hline N1D & C8D & C7D & $105.68(19)$ \\
\hline N1D & C9D & C10D & $112.3(2)$ \\
\hline C11D & C10D & C9D & $120.5(2)$ \\
\hline C11D & C10D & C17D & $122.3(2)$ \\
\hline C17D & C10D & C9D & $117.2(2)$ \\
\hline C12D & C11D & C10D & $179.4(2)$ \\
\hline C11D & C12D & C13D & $126.6(2)$ \\
\hline C12D & C13D & C14D & $111.5(2)$ \\
\hline C12D & C13D & C15D & $108.2(2)$ \\
\hline C12D & C13D & C16D & $107.0(2)$ \\
\hline C14D & C13D & C15D & $111.0(3)$ \\
\hline C14D & C13D & C16D & $109.6(2)$ \\
\hline C16D & C13D & C15D & $109.4(2)$ \\
\hline C18D & C17D & C10D & $122.2(2)$ \\
\hline C18D & C17D & C22D & $118.7(2)$ \\
\hline C22D & C17D & C10D & $119.0(2)$ \\
\hline
\end{tabular}




\begin{tabular}{|c|c|c|c|}
\hline $\mathrm{C} 19 \mathrm{C}$ & $\mathrm{C} 18 \mathrm{C}$ & $\mathrm{C} 17 \mathrm{C}$ & $121.0(2)$ \\
\hline $\mathrm{O} 3 \mathrm{C}$ & C19C & $\mathrm{C} 18 \mathrm{C}$ & $124.8(2)$ \\
\hline $\mathrm{O} 3 \mathrm{C}$ & $\mathrm{C} 19 \mathrm{C}$ & $\mathrm{C} 20 \mathrm{C}$ & $115.7(2)$ \\
\hline $\mathrm{C} 18 \mathrm{C}$ & C19C & C20C & $119.5(2)$ \\
\hline $\mathrm{O} 4 \mathrm{C}$ & C20C & C19C & $115.4(2)$ \\
\hline $\mathrm{O} 4 \mathrm{C}$ & $\mathrm{C} 20 \mathrm{C}$ & $\mathrm{C} 21 \mathrm{C}$ & $124.9(2)$ \\
\hline $\mathrm{C} 21 \mathrm{C}$ & $\mathrm{C} 20 \mathrm{C}$ & $\mathrm{C} 19 \mathrm{C}$ & $119.7(2)$ \\
\hline $\mathrm{C} 20 \mathrm{C}$ & $\mathrm{C} 21 \mathrm{C}$ & $\mathrm{C} 22 \mathrm{C}$ & $120.4(2)$ \\
\hline $\mathrm{C} 17 \mathrm{C}$ & $\mathrm{C} 22 \mathrm{C}$ & $\mathrm{C} 21 \mathrm{C}$ & $120.5(2)$ \\
\hline $\mathrm{C} 21 \mathrm{~A}$ & $\mathrm{O} 3 \mathrm{~A}$ & $\mathrm{C} 23 \mathrm{~A}$ & $116.27(18)$ \\
\hline $\mathrm{C} 20 \mathrm{~A}$ & $\mathrm{O} 4 \mathrm{~A}$ & $\mathrm{C} 24 \mathrm{~A}$ & $116.83(18)$ \\
\hline C1A & N1A & C8A & $112.1(2)$ \\
\hline $\mathrm{C} 1 \mathrm{~A}$ & N1A & C9A & $122.9(2)$ \\
\hline C8A & N1A & C9A & $124.6(2)$ \\
\hline O1A & C1A & N1A & $124.5(2)$ \\
\hline O1A & $\mathrm{C} 1 \mathrm{~A}$ & $\mathrm{C} 2 \mathrm{~A}$ & $129.4(2)$ \\
\hline N1A & $\mathrm{C} 1 \mathrm{~A}$ & $\mathrm{C} 2 \mathrm{~A}$ & $106.1(2)$ \\
\hline C3A & $\mathrm{C} 2 \mathrm{~A}$ & $\mathrm{C} 1 \mathrm{~A}$ & $130.2(2)$ \\
\hline C3A & $\mathrm{C} 2 \mathrm{~A}$ & C7A & $122.0(2)$ \\
\hline C7A & $\mathrm{C} 2 \mathrm{~A}$ & $\mathrm{C} 1 \mathrm{~A}$ & $107.8(2)$ \\
\hline $\mathrm{C} 2 \mathrm{~A}$ & C3A & C4A & $116.9(2)$ \\
\hline C5A & $\mathrm{C} 4 \mathrm{~A}$ & C3A & $120.7(2)$ \\
\hline C6A & C5A & C4A & $121.9(2)$ \\
\hline C7A & C6A & C5A & $117.1(2)$ \\
\hline $\mathrm{C} 2 \mathrm{~A}$ & C7A & C8A & $108.8(2)$ \\
\hline C6A & C7A & $\mathrm{C} 2 \mathrm{~A}$ & $121.5(2)$ \\
\hline C6A & C7A & C8A & $129.7(2)$ \\
\hline $\mathrm{O} 2 \mathrm{~A}$ & C8A & N1A & $124.6(2)$ \\
\hline $\mathrm{O} 2 \mathrm{~A}$ & C8A & C7A & 130.1(2) \\
\hline N1A & C8A & C7A & $105.2(2)$ \\
\hline N1A & C9A & $\mathrm{C} 10 \mathrm{~A}$ & $111.79(19)$ \\
\hline $\mathrm{C} 11 \mathrm{~A}$ & $\mathrm{C} 10 \mathrm{~A}$ & C9A & $119.8(2)$ \\
\hline $\mathrm{C} 11 \mathrm{~A}$ & $\mathrm{C} 10 \mathrm{~A}$ & C17A & $122.7(2)$ \\
\hline C17A & C10A & C9A & $117.44(19)$ \\
\hline $\mathrm{C} 12 \mathrm{~A}$ & C11A & $\mathrm{C} 10 \mathrm{~A}$ & 178.5(3) \\
\hline $\mathrm{C} 11 \mathrm{~A}$ & C12A & C13A & $125.7(2)$ \\
\hline $\mathrm{C} 12 \mathrm{~A}$ & $\mathrm{C} 13 \mathrm{~A}$ & C14A & $109.2(2)$ \\
\hline $\mathrm{C} 12 \mathrm{~A}$ & C13A & $\mathrm{C} 15 \mathrm{~A}$ & $107.5(2)$ \\
\hline $15 \mathrm{~A}$ & C13A & C14A & $108.6(2)$ \\
\hline
\end{tabular}

\begin{tabular}{|c|c|c|c|}
\hline C17D & C18D & C19D & $120.9(2)$ \\
\hline C20D & C19D & C18D & $120.6(2)$ \\
\hline O3D & C20D & C19D & $125.4(2)$ \\
\hline O3D & C20D & C21D & $115.7(2)$ \\
\hline C19D & C20D & C21D & $118.9(2)$ \\
\hline O4D & C21D & C20D & $115.4(2)$ \\
\hline C22D & C21D & O4D & $124.2(2)$ \\
\hline C22D & C21D & C20D & $120.4(2)$ \\
\hline C21D & C22D & C17D & $120.6(2)$ \\
\hline C19B & O3B & C24B & $116.70(17)$ \\
\hline C20B & O4B & $\mathrm{C} 23 \mathrm{~B}$ & $116.82(17)$ \\
\hline $\mathrm{C} 1 \mathrm{~B}$ & N1B & C9B & $123.3(2)$ \\
\hline C8B & N1B & $\mathrm{C} 1 \mathrm{~B}$ & $112.7(2)$ \\
\hline $88 \mathrm{~B}$ & N1B & C9B & $123.6(2)$ \\
\hline O1B & C1B & N1B & $125.1(2)$ \\
\hline O1B & $\mathrm{C} 1 \mathrm{~B}$ & $\mathrm{C} 2 \mathrm{~B}$ & $129.1(2)$ \\
\hline N1B & C1B & $\mathrm{C} 2 \mathrm{~B}$ & $105.8(2)$ \\
\hline C3B & $\mathrm{C} 2 \mathrm{~B}$ & $\mathrm{C} 1 \mathrm{~B}$ & $130.8(2)$ \\
\hline C3B & $\mathrm{C} 2 \mathrm{~B}$ & C7B & $121.5(2)$ \\
\hline C7B & $\mathrm{C} 2 \mathrm{~B}$ & C1B & $107.7(2)$ \\
\hline $\mathrm{C} 2 \mathrm{~B}$ & C3B & C4B & $116.9(3)$ \\
\hline $\mathrm{C} 5 \mathrm{~B}$ & C4B & C3B & $121.6(3)$ \\
\hline C4B & C5B & C6B & $121.2(3)$ \\
\hline C7B & C6B & C5B & $116.8(3)$ \\
\hline $\mathrm{C} 2 \mathrm{~B}$ & C7B & C8B & $108.2(2)$ \\
\hline C6B & C7B & $\mathrm{C} 2 \mathrm{~B}$ & $122.0(2)$ \\
\hline C6B & C7B & C8B & $129.8(3)$ \\
\hline $\mathrm{O} 2 \mathrm{~B}$ & C8B & N1B & $126.0(2)$ \\
\hline $\mathrm{O} 2 \mathrm{~B}$ & C8B & C7B & $128.5(2)$ \\
\hline N1B & C8B & C7B & $105.6(2)$ \\
\hline N1B & C9B & C10B & $111.56(19)$ \\
\hline $\mathrm{C} 11 \mathrm{~B}$ & C10B & C9B & $119.6(2)$ \\
\hline $\mathrm{C} 11 \mathrm{~B}$ & C10B & $\mathrm{C} 17 \mathrm{~B}$ & $122.3(2)$ \\
\hline C17B & C10B & C9B & $117.8(2)$ \\
\hline $\mathrm{C} 12 \mathrm{~B}$ & C11B & $\mathrm{C} 10 \mathrm{~B}$ & $175.0(2)$ \\
\hline C11B & C12B & C13B & $126.6(2)$ \\
\hline C12B & C13B & C14B & $107.8(2)$ \\
\hline $12 \mathrm{~B}$ & C13B & C15B & $109.11(19)$ \\
\hline 121 & C13B & C16B & $111.1(2)$ \\
\hline
\end{tabular}




$\begin{array}{llll}\text { C16A } & \text { C13A } & \text { C12A } 111.2(2) \\ \text { C16A } & \text { C13A } & \text { C14A } 110.0(2) \\ \text { C16A } & \text { C13A } & \text { C15A } 110.2(2) \\ \text { C18A } & \text { C17A } & \text { C10A } 121.9(2) \\ \text { C18A } & \text { C17A } & \text { C22A } 118.6(2) \\ \text { C22A } & \text { C17A } & \text { C10A } 119.5(2) \\ \text { C17A } & \text { C18A } & \text { C19A } 120.8(2) \\ \text { C20A } & \text { C19A } & \text { C18A } 120.4(2) \\ \text { O4A } & \text { C20A } & \text { C19A } 124.9(2) \\ \text { O4A } & \text { C20A } & \text { C21A } 115.6(2) \\ \text { C19A } & \text { C20A } & \text { C21A } 119.4(2) \\ \text { O3A } & \text { C21A } & \text { C20A } 115.5(2) \\ \text { O3A } & \text { C21A } & \text { C22A } 124.6(2) \\ \text { C22A } & \text { C21A } & \text { C20A } 119.9(2) \\ \text { C21A } & \text { C22A } & \text { C17A } 121.0(2)\end{array}$

Table A20. Torsion Angles.

\begin{tabular}{|c|c|c|c|c|c|c|c|c|c|}
\hline $\mathbf{A}$ & B & $\mathbf{C}$ & D & Angle ${ }^{\circ}$ & $\mathbf{A}$ & B & $\mathbf{C}$ & D & Angle $/^{\circ}$ \\
\hline $\mathrm{O} 1 \mathrm{C}$ & $\mathrm{C} 1 \mathrm{C}$ & $\mathrm{C} 2 \mathrm{C}$ & $\mathrm{C} 3 \mathrm{C}$ & $-2.2(5)$ & O1D & C1D & $\mathrm{C} 2 \mathrm{D}$ & C3D & $2.1(5)$ \\
\hline $\mathrm{O} 1 \mathrm{C}$ & $\mathrm{C} 1 \mathrm{C}$ & $\mathrm{C} 2 \mathrm{C}$ & $\mathrm{C} 7 \mathrm{C}$ & $179.4(3)$ & O1D & C1D & $\mathrm{C} 2 \mathrm{D}$ & C7D & $-178.6(3)$ \\
\hline $\mathrm{O} 3 \mathrm{C}$ & $\mathrm{C} 19 \mathrm{C}$ & C20C & $\mathrm{O} 4 \mathrm{C}$ & $3.3(3)$ & O3D & C20D & C21D & O4D & $-2.1(3)$ \\
\hline $\mathrm{O} 3 \mathrm{C}$ & $\mathrm{C} 19 \mathrm{C}$ & $\mathrm{C} 20 \mathrm{C}$ & $\mathrm{C} 21 \mathrm{C}$ & $-174.2(2)$ & O3D & C20D & C21D & $\mathrm{C} 22 \mathrm{D}$ & $-179.6(2)$ \\
\hline $\mathrm{O} 4 \mathrm{C}$ & $\mathrm{C} 20 \mathrm{C}$ & $\mathrm{C} 21 \mathrm{C}$ & $\mathrm{C} 22 \mathrm{C}$ & $180.0(2)$ & O4D & $\mathrm{C} 21 \mathrm{D}$ & C22D & C17D & $-175.90(19)$ \\
\hline $\mathrm{N} 1 \mathrm{C}$ & $\mathrm{C} 1 \mathrm{C}$ & $\mathrm{C} 2 \mathrm{C}$ & $\mathrm{C} 3 \mathrm{C}$ & $178.5(3)$ & N1D & C1D & $\mathrm{C} 2 \mathrm{D}$ & C3D & $-178.7(3)$ \\
\hline $\mathrm{N} 1 \mathrm{C}$ & $\mathrm{C} 1 \mathrm{C}$ & $\mathrm{C} 2 \mathrm{C}$ & $\mathrm{C} 7 \mathrm{C}$ & $0.0(3)$ & N1D & C1D & $\mathrm{C} 2 \mathrm{D}$ & C7D & $0.6(3)$ \\
\hline $\mathrm{N} 1 \mathrm{C}$ & $\mathrm{C} 9 \mathrm{C}$ & $\mathrm{C} 10 \mathrm{C}$ & $\mathrm{C} 11 \mathrm{C}$ & $4.5(3)$ & N1D & C9D & C10D & C11D & $14.7(3)$ \\
\hline $\mathrm{N} 1 \mathrm{C}$ & $\mathrm{C} 9 \mathrm{C}$ & $\mathrm{C} 10 \mathrm{C}$ & $\mathrm{C} 17 \mathrm{C}$ & $177.4(2)$ & N1D & C9D & C10D & C17D & $-165.76(19)$ \\
\hline $\mathrm{C} 1 \mathrm{C}$ & $\mathrm{N} 1 \mathrm{C}$ & $\mathrm{C} 8 \mathrm{C}$ & $\mathrm{O} 2 \mathrm{C}$ & $178.5(2)$ & C1D & N1D & C8D & $\mathrm{O} 2 \mathrm{D}$ & $-179.2(2)$ \\
\hline $\mathrm{C} 1 \mathrm{C}$ & $\mathrm{N} 1 \mathrm{C}$ & $\mathrm{C} 8 \mathrm{C}$ & $\mathrm{C} 7 \mathrm{C}$ & $-1.4(3)$ & C1D & N1D & C8D & C7D & $0.5(3)$ \\
\hline $\mathrm{C} 1 \mathrm{C}$ & $\mathrm{N} 1 \mathrm{C}$ & C9C & $\mathrm{C} 10 \mathrm{C}$ & $103.7(3)$ & C1D & N1D & C9D & C10D & $-88.7(3)$ \\
\hline $\mathrm{C} 1 \mathrm{C}$ & $\mathrm{C} 2 \mathrm{C}$ & $\mathrm{C} 3 \mathrm{C}$ & $\mathrm{C} 4 \mathrm{C}$ & $-179.2(2)$ & C1D & $\mathrm{C} 2 \mathrm{D}$ & $\mathrm{C} 3 \mathrm{D}$ & C4D & $-179.5(2)$ \\
\hline $\mathrm{C} 1 \mathrm{C}$ & $\mathrm{C} 2 \mathrm{C}$ & C7C & C6C & $179.0(2)$ & C1D & C2D & C7D & C6D & $179.7(2)$ \\
\hline $\mathrm{C} 1 \mathrm{C}$ & $\mathrm{C} 2 \mathrm{C}$ & C7C & $\mathrm{C} 8 \mathrm{C}$ & $-0.9(3)$ & C1D & $\mathrm{C} 2 \mathrm{D}$ & C7D & C8D & $-0.3(3)$ \\
\hline $\mathrm{C} 2 \mathrm{C}$ & $\mathrm{C} 3 \mathrm{C}$ & $\mathrm{C} 4 \mathrm{C}$ & C5C & $0.6(4)$ & $\mathrm{C} 2 \mathrm{D}$ & C3D & C4D & C5D & $-0.7(4)$ \\
\hline $\mathrm{C} 2 \mathrm{C}$ & C7C & $\mathrm{C} 8 \mathrm{C}$ & $\mathrm{O} 2 \mathrm{C}$ & $-178.5(3)$ & $\mathrm{C} 2 \mathrm{D}$ & C7D & C8D & $\mathrm{O} 2 \mathrm{D}$ & $179.5(3)$ \\
\hline $\mathrm{C} 2 \mathrm{C}$ & C7C & $\mathrm{C} 8 \mathrm{C}$ & $\mathrm{N} 1 \mathrm{C}$ & $1.4(3)$ & $\mathrm{C} 2 \mathrm{D}$ & C7D & C8D & N1D & $-0.1(3)$ \\
\hline $\mathrm{C} 3 \mathrm{C}$ & $\mathrm{C} 2 \mathrm{C}$ & C7C & C6C & $0.5(4)$ & C3D & C2D & C7D & C6D & $-0.9(4)$ \\
\hline $\mathrm{C} 3 \mathrm{C}$ & $\mathrm{C} 2 \mathrm{C}$ & C7C & $\mathrm{C} 8 \mathrm{C}$ & $-179.4(2)$ & C3D & $\mathrm{C} 2 \mathrm{D}$ & C7D & C8D & 179.1(2) \\
\hline $\mathrm{C} 3 \mathrm{C}$ & $\mathrm{C} 4 \mathrm{C}$ & C5C & C6C & $0.3(4)$ & $\begin{array}{r}\text { C3D } \\
S-50\end{array}$ & C4D & C5D & C6D & $-0.3(4)$ \\
\hline
\end{tabular}

$\begin{array}{llll}\text { C14B } & \text { C13B } & \text { C15B } & 109.4(2) \\ \text { C16B } & \text { C13B } & \text { C14B } & 109.6(2) \\ \text { C16B } & \text { C13B } & \text { C15B } & 109.9(2) \\ \text { C18B } & \text { C17B } & \text { C10B } & 120.0(2) \\ \text { C22B } & \text { C17B } & \text { C10B } & 121.2(2) \\ \text { C22B } & \text { C17B } & \text { C18B } & 118.6(2) \\ \text { C19B } & \text { C18B } & \text { C17B } & 120.9(2) \\ \text { O3B } & \text { C19B } & \text { C18B } & 124.7(2) \\ \text { O3B } & \text { C19B } & \text { C20B } & 115.4(2) \\ \text { C18B } & \text { C19B } & \text { C20B } & 119.9(2) \\ \text { O4B } & \text { C20B } & \text { C19B } & 115.84(19) \\ \text { O4B } & \text { C20B } & \text { C21B } & 125.0(2) \\ \text { C21B } & \text { C20B } & \text { C19B } & 119.1(2) \\ \text { C20B } & \text { C21B } & \text { C22B } & 120.6(2) \\ \text { C17B } & \text { C22B } & \text { C21B } & 120.6(2)\end{array}$




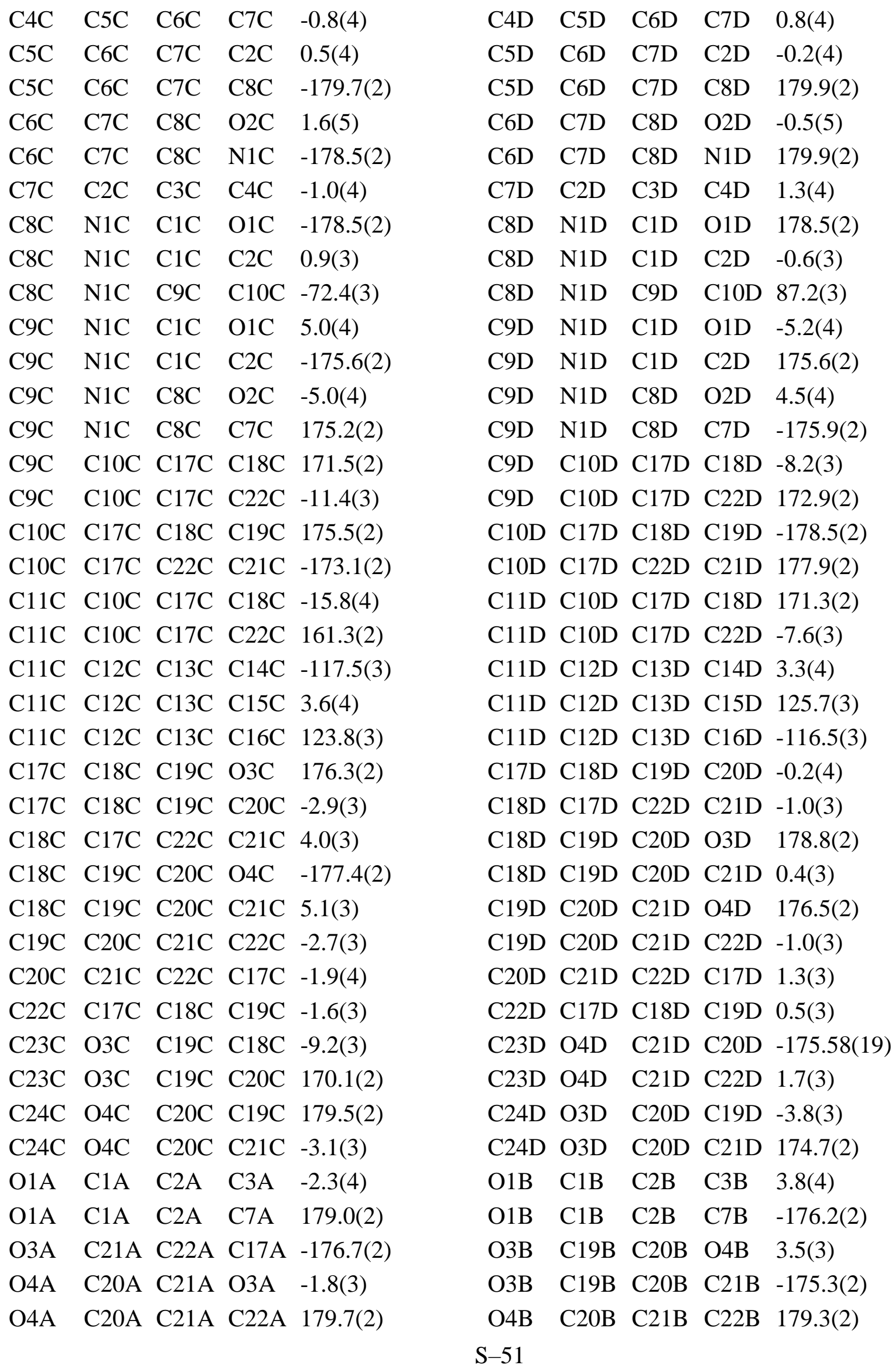


N1A $\quad$ C1A $\quad$ C2A $\quad$ C3A $178.2(2)$

N1A C1A C2A C7A $-0.5(2)$

N1A C9A C10A C11A 15.6(3)

N1A C9A C10A C17A -167.4(2)

C1A N1A C8A O2A $-179.7(2)$

C1A N1A C8A C7A $-0.3(2)$

C1A N1A C9A C10A 87.0(3)

C1A C2A C3A C4A $\quad$-179.3(2)

C1A C2A C7A C6A 179.3(2)

C1A $\quad$ C2A $\quad$ C7A $\quad$ C8A $0.3(2)$

C2A C3A C4A C5A $0.4(4)$

C2A $\quad$ C7A $\quad$ C8A $\quad$ O2A $179.4(2)$

C2A C7A C8A N1A $0.0(2)$

C3A $\quad$ C2A $\quad$ C7A $\quad$ C6A $0.5(3)$

C3A C2A C7A $\quad$ C8A $-178.5(2)$

C3A C4A C5A C6A $0.2(4)$

C4A C5A $\quad$ C6A $\quad$ C7A $-0.4(4)$

C5A C6A C7A C2A $0.1(3)$

C5A C6A C7A C8A 178.9(2)

C6A $\quad$ C7A $\quad$ C8A $\quad$ O2A $0.5(4)$

C6A C7A C8A N1A $-179.0(2)$

C7A $\quad$ C2A $\quad$ C3A $\quad$ C4A $-0.8(3)$

C8A N1A C1A O1A $-179.0(2)$

C8A N1A C1A C2A $0.5(2)$

C8A N1A C9A C10A $-84.8(3)$

C9A N1A C1A O1A 8.3(3)

C9A N1A C1A C2A $-172.24(19)$

C9A N1A C8A O2A

C9A N1A C8A C7A $172.29(19)$

C9A C10A C17A C18A -7.7(4)

C9A C10A C17A C22A 172.8(2)

C10A C17A C18A C19A -178.8(2)

C10A C17A C22A C21A 178.0(2)

C11A C10A C17A C18A 169.1(3)

C11A C10A C17A C22A -10.3(4)

C11A C12A C13A C14A 116.8(3)

C11A C12A C13A C15A -125.6(3)

C11A C12A C13A C16A -4.8(3)

C17A C18A C19A C20A 0.0(4)
N1B $\quad$ C1B $\quad$ C2B $\quad$ C3B $\quad-177.0(2)$

N1B C1B C2B C7B 3.0(2)

N1B C9B C10B C11B 8.0(3)

N1B C9B C10B C17B $-177.8(2)$

$\mathrm{C} 1 \mathrm{~B} \quad \mathrm{~N} 1 \mathrm{~B} \quad \mathrm{C} 8 \mathrm{~B} \quad \mathrm{O} 2 \mathrm{~B} \quad 179.4(2)$

C1B N1B $\quad$ C8B $\quad$ C7B $0.3(2)$

C1B N1B C9B C10B $-73.2(3)$

C1B C2B C3B C4B $\quad$-179.3(2)

C1B $\quad$ C2B $\quad$ C7B $\quad$ C6B $178.2(2)$

$\mathrm{C} 1 \mathrm{~B} \quad \mathrm{C} 2 \mathrm{~B} \quad \mathrm{C} 7 \mathrm{~B} \quad \mathrm{C} 8 \mathrm{~B} \quad-2.8(2)$

C2B $\quad$ C3B $\quad$ C4B $\quad$ C5B $0.6(4)$

C2B $\quad$ C7B $\quad$ C8B $\quad$ O2B $\quad-177.5(2)$

C2B C7B C8B N1B 1.7(2)

C3B $\quad$ C2B $\quad$ C7B $\quad$ C6B $-1.8(4)$

C3B $\quad$ C2B $\quad$ C7B $\quad$ C8B $177.2(2)$

C3B C4B C5B C6B $-0.8(4)$

C4B $\quad$ C5B $\quad$ C6B $\quad$ C7B $-0.2(4)$

C5B C6B $\quad$ C7B $\quad$ C2B $1.5(4)$

C5B $\quad$ C6B $\quad$ C7B $\quad$ C8B $-177.2(2)$

C6B $\quad \mathrm{C} 7 \mathrm{~B} \quad \mathrm{C} 8 \mathrm{~B} \quad \mathrm{O} 2 \mathrm{~B} \quad 1.4(4)$

C6B C7B C8B N1B $\quad-179.5(2)$

C7B $\quad$ C2B $\quad$ C3B $\quad$ C4B $0.6(4)$

C8B N1B $\quad$ C1B $\quad$ O1B $177.3(2)$

C8B N1B C1B C2B $-2.0(2)$

C8B N1B C9B C10B 98.0(3)

C9B N1B C1B O1B $-10.7(3)$

C9B N1B $\quad$ C1B $\quad$ C2B $170.1(2)$

C9B N1B $\quad$ C8B $\quad$ O2B $7.4(4)$

C9B N1B C8B C7B $\quad-171.7(2)$

C9B C10B C17B C18B 168.3(2)

C9B C10B C17B C22B -16.0(3)

C10B C17B C18B C19B 174.8(2)

C10B C17B C22B C21B -173.1(2)

C11B C10B C17B C18B -17.7(4)

C11B C10B C17B C22B 158.0(2)

C11B C12B C13B C14B -112.6(3)

C11B C12B C13B C15B 128.7(2)

C11B C12B C13B C16B 7.4(3)

C17B C18B C19B O3B 176.7(2) 


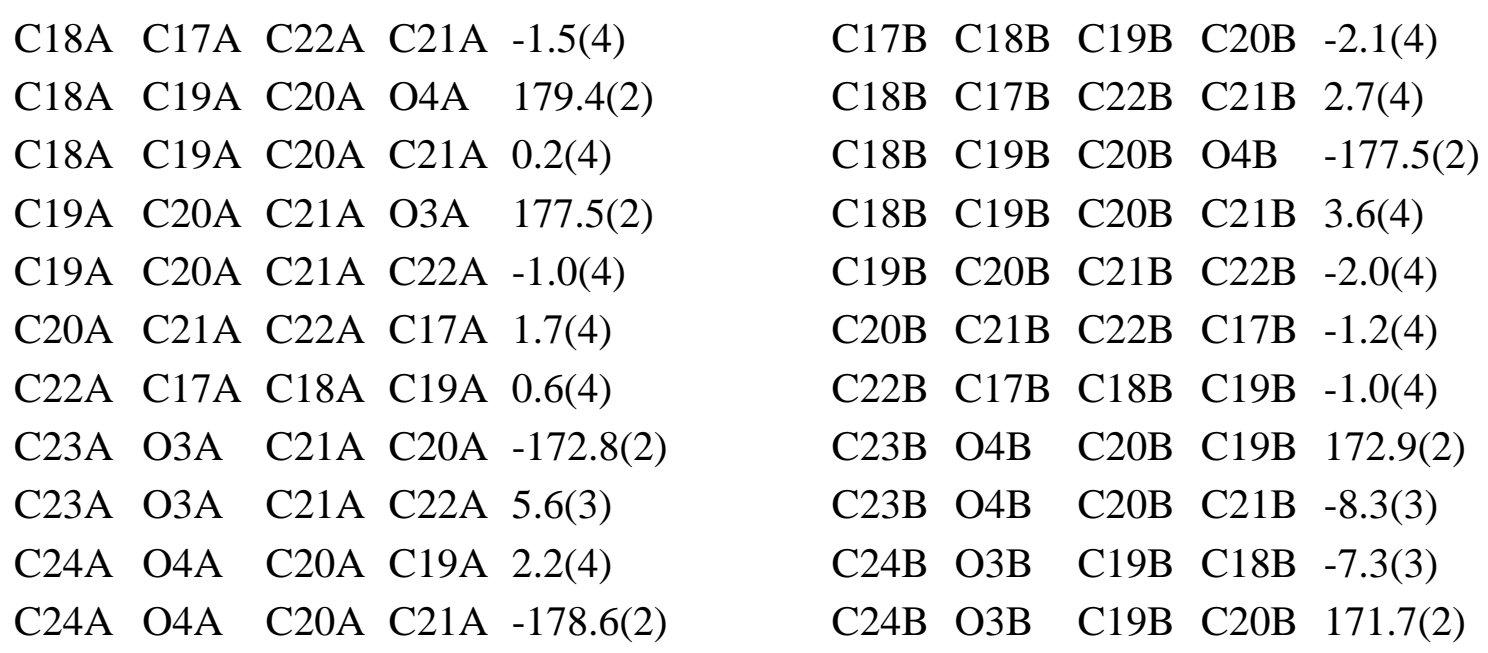

Table A21. Hydrogen Atom Coordinates $\left(\AA \times 10^{4}\right)$ and Isotropic Displacement Parameters $\left(\AA^{2} \times 10^{3}\right)$.

$\begin{array}{lllll}\text { Atom } & \boldsymbol{x} & \boldsymbol{y} & \boldsymbol{z} & \mathbf{U}(\mathbf{e q}) \\ \text { H3C } & 5042 & 737 & 5583 & 29 \\ \text { H4C } & 5935 & 61 & 7003 & 30 \\ \text { H5C } & 6193 & 1277 & 8068 & 28 \\ \text { H6C } & 5547 & 3238 & 7756 & 26 \\ \text { H9CA } & 3350 & 4855 & 3993 & 28 \\ \text { H9CB } & 3841 & 5622 & 4546 & 28 \\ \text { H12C } & 1863 & 5653 & 7275 & 31 \\ \text { H14A } & -447 & 4738 & 7834 & 72 \\ \text { H14B } & -587 & 5810 & 7182 & 72 \\ \text { H14C } & 71 & 5619 & 8138 & 72 \\ \text { H15A } & 1535 & 3571 & 5816 & 68 \\ \text { H15B } & 332 & 4621 & 5756 & 68 \\ \text { H15C } & 414 & 3530 & 6362 & 68 \\ \text { H16A } & 1951 & 3947 & 8238 & 67 \\ \text { H16B } & 2520 & 3164 & 7318 & 67 \\ \text { H16C } & 1408 & 3113 & 7872 & 67 \\ \text { H18C } & 27 & 7168 & 5337 & 22 \\ \text { H21C } & 1694 & 8626 & 2898 & 25 \\ \text { H22C } & 2761 & 6877 & 3568 & 25 \\ \text { H23A } & -1900 & 7799 & 4933 & 38 \\ \text { H23B } & -2818 & 9011 & 5291 & 38 \\ \text { H23C } & -1687 & 8390 & 5847 & 38 \\ \text { H24A } & 246 & 9977 & 2068 & 43 \\ \text { H24B } & 708 & 10508 & 2879 & 43 \\ \text { H24C } & -525 & 11209 & 2496 & 43 \\ & & & & \text { S-53 }\end{array}$




\begin{tabular}{|c|c|c|c|c|}
\hline $\mathrm{H} 3 \mathrm{~A}$ & 5284 & 9826 & -924 & 27 \\
\hline $\mathrm{H} 4 \mathrm{~A}$ & 3942 & 11697 & -1145 & 32 \\
\hline H5A & 2059 & 12250 & -552 & 32 \\
\hline H6A & 1438 & 10982 & 283 & 30 \\
\hline H9AA & 4894 & 6131 & 667 & 26 \\
\hline H9AB & 3597 & 6498 & 1074 & 26 \\
\hline $\mathrm{H} 12 \mathrm{~A}$ & 3418 & 9108 & 3170 & 29 \\
\hline H14D & 3649 & 10897 & 2560 & 62 \\
\hline $\mathrm{H} 14 \mathrm{E}$ & 4401 & 10303 & 1615 & 62 \\
\hline $\mathrm{H} 14 \mathrm{~F}$ & 4891 & 10904 & 2348 & 62 \\
\hline H15D & 5582 & 10013 & 3864 & 58 \\
\hline $\mathrm{H} 15 \mathrm{E}$ & 5394 & 8903 & 4207 & 58 \\
\hline $\mathrm{H} 15 \mathrm{~F}$ & 4322 & 10088 & 4149 & 58 \\
\hline H16D & 6017 & 8469 & 1689 & 61 \\
\hline H16E & 6428 & 7900 & 2691 & 61 \\
\hline $\mathrm{H} 16 \mathrm{~F}$ & 6615 & 9017 & 2372 & 61 \\
\hline H18A & 4959 & 4650 & 1565 & 26 \\
\hline H19A & 5827 & 2955 & 2385 & 26 \\
\hline $\mathrm{H} 22 \mathrm{~A}$ & 5600 & 6363 & 3677 & 22 \\
\hline $\mathrm{H} 23 \mathrm{D}$ & 6737 & 6069 & 4869 & 36 \\
\hline $\mathrm{H} 23 \mathrm{E}$ & 6649 & 5520 & 5883 & 36 \\
\hline $\mathrm{H} 23 \mathrm{~F}$ & 5528 & 6132 & 5312 & 36 \\
\hline $\mathrm{H} 24 \mathrm{D}$ & 7282 & 1515 & 3051 & 40 \\
\hline $\mathrm{H} 24 \mathrm{E}$ & 6086 & 1655 & 3580 & 40 \\
\hline $\mathrm{H} 24 \mathrm{~F}$ & 7244 & 1035 & 4103 & 40 \\
\hline H3D & -1135 & 12970 & 8020 & 28 \\
\hline H4D & -1976 & 13670 & 6583 & 32 \\
\hline H5D & -2208 & 12468 & 5479 & 30 \\
\hline H6D & -1590 & 10519 & 5774 & 28 \\
\hline H9DA & 76 & 7992 & 8852 & 26 \\
\hline H9DB & 247 & 8801 & 9623 & 26 \\
\hline H12D & 2881 & 9665 & 8026 & 25 \\
\hline $\mathrm{H} 14 \mathrm{G}$ & 2705 & 8222 & 5478 & 65 \\
\hline $\mathrm{H} 14 \mathrm{H}$ & 1644 & 8604 & 6252 & 65 \\
\hline H14I & 2797 & 7544 & 6454 & 65 \\
\hline $\mathrm{H} 15 \mathrm{G}$ & 2698 & 10767 & 6525 & 74 \\
\hline $\mathrm{H} 15 \mathrm{H}$ & 1568 & 10576 & 6331 & 74 \\
\hline H15I & 2597 & 10248 & 5529 & 74 \\
\hline $\mathrm{H} 16 \mathrm{G}$ & 4519 & 9100 & 6776 & 58 \\
\hline
\end{tabular}




$\begin{array}{lllll}\text { H16H } & 4429 & 8607 & 5764 & 58 \\ \text { H16I } & 4565 & 7844 & 6694 & 58 \\ \text { H18D } & 1039 & 6739 & 9922 & 25 \\ \text { H19D } & 2106 & 4951 & 10519 & 26 \\ \text { H22D } & 3833 & 6662 & 8340 & 22 \\ \text { H23G } & 5500 & 5376 & 7717 & 35 \\ \text { H23H } & 6647 & 4751 & 8252 & 35 \\ \text { H23I } & 5732 & 5957 & 8628 & 35 \\ \text { H24G } & 3187 & 3066 & 10591 & 45 \\ \text { H24H } & 3554 & 3696 & 11392 & 45 \\ \text { H24I } & 4411 & 2465 & 11018 & 45 \\ \text { H3B } & 8651 & 3824 & 4474 & 31 \\ \text { H4B } & 10003 & 1955 & 4621 & 36 \\ \text { H5B } & 11868 & 1423 & 4008 & 37 \\ \text { H6B } & 12456 & 2733 & 3185 & 32 \\ \text { H9BA } & 10286 & 7104 & 2285 & 28 \\ \text { H9BB } & 9121 & 7595 & 2942 & 28 \\ \text { H12B } & 7521 & 5504 & 1753 & 24 \\ \text { H14J } & 7891 & 5098 & -403 & 45 \\ \text { H14K } & 7214 & 4523 & 266 & 45 \\ \text { H14L } & 8291 & 3751 & -409 & 45 \\ \text { H15J } & 8122 & 3408 & 1692 & 46 \\ \text { H15K } & 9396 & 3234 & 1894 & 46 \\ \text { H15L } & 9172 & 2625 & 997 & 46 \\ \text { H16J } & 10238 & 3552 & -68 & 50 \\ \text { H16K } & 10438 & 4126 & 862 & 50 \\ \text { H16L } & 9849 & 4897 & -13 & 50 \\ \text { H18B } & 8126 & 7386 & -96 & 22 \\ \text { H21B } & 8133 & 10738 & 1121 & 25 \\ \text { H22B } & 8897 & 9062 & 1974 & 25 \\ \text { H23J } & 6646 & 12682 & -569 & 40 \\ \text { H23K } & 7787 & 12083 & -20 & 40 \\ \text { H23L } & 6587 & 12181 & 471 & 40 \\ \text { H24J } & 7264 & 8216 & -2371 & 33 \\ \text { H24K } & 7030 & 7722 & -1366 & 33 \\ \text { H24 } & 8301 & 7558 & -1721 & 33\end{array}$




\section{SFC Traces}

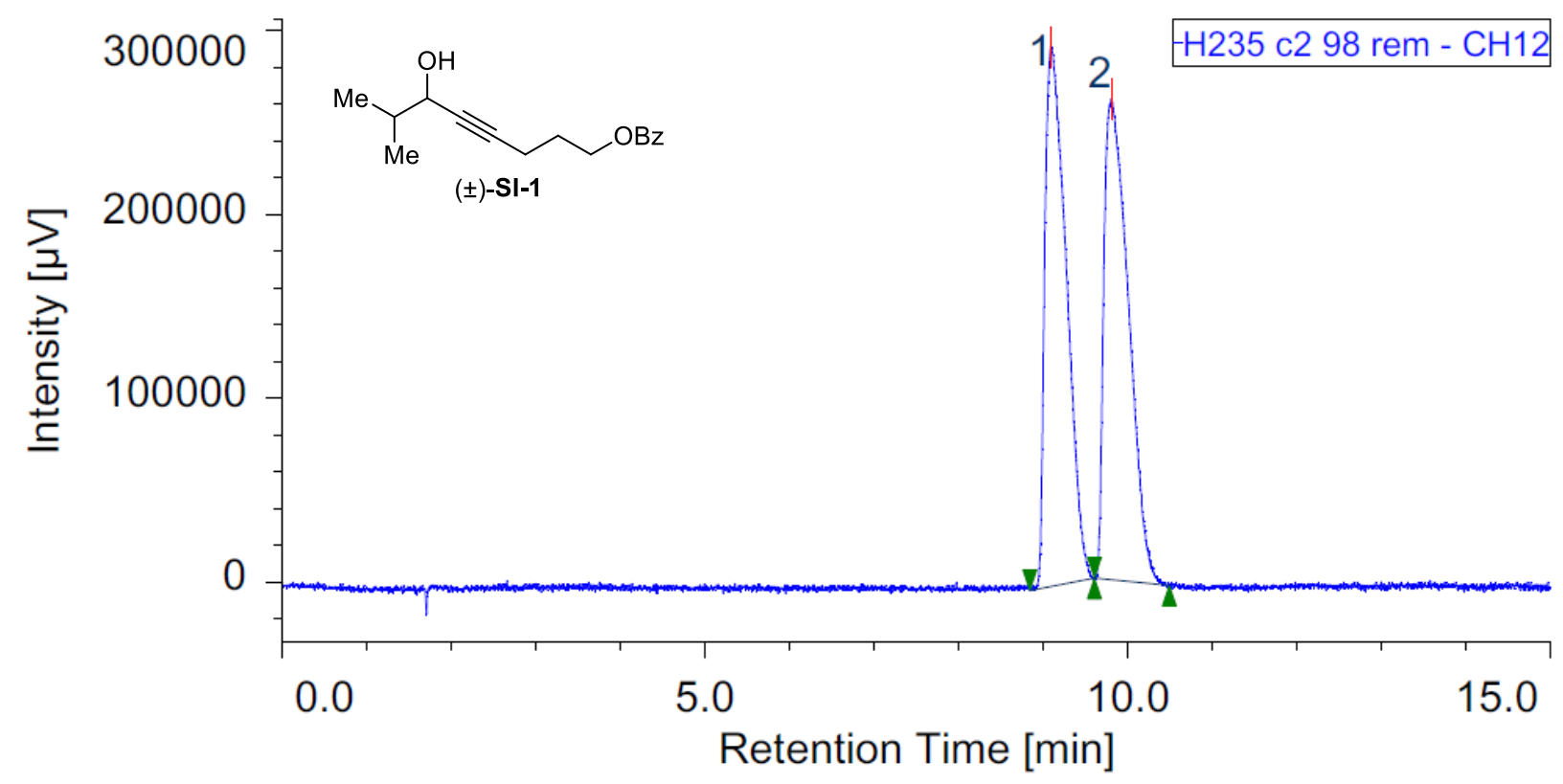

\begin{tabular}{|c|c|c|c|c|c|c|c|c|c|c|}
\hline \begin{tabular}{|l|l|l|}
$\#$ Peak Name \\
\end{tabular} & $\mathrm{CH}$ & $t R[\mathrm{~min}]$ & Area $[\mu \mathrm{V} \cdot \mathrm{sec}]$ & Height $[\mu \mathrm{V}]$ & Area $\%$ & Height $\%$ & \begin{tabular}{|l|} 
Quantity \\
\end{tabular} & NTP & \begin{tabular}{|l|} 
Resolution \\
\end{tabular} & Symmetry Factor \\
\hline Unknown & 12 & 9.093 & 5160990 & 293138 & 49.52 & 52.88 & $\mathrm{~N} / \mathrm{A}$ & 539. & 1.338 & 1.933 \\
\hline Unknown & 12 & 9.800 & 5259778 & 261197 & 50.47 & 47.119 & $\mathrm{~N} / \mathrm{A}$ & 483 & $\mathrm{~N} / \mathrm{A}$ & 2.018 \\
\hline
\end{tabular}

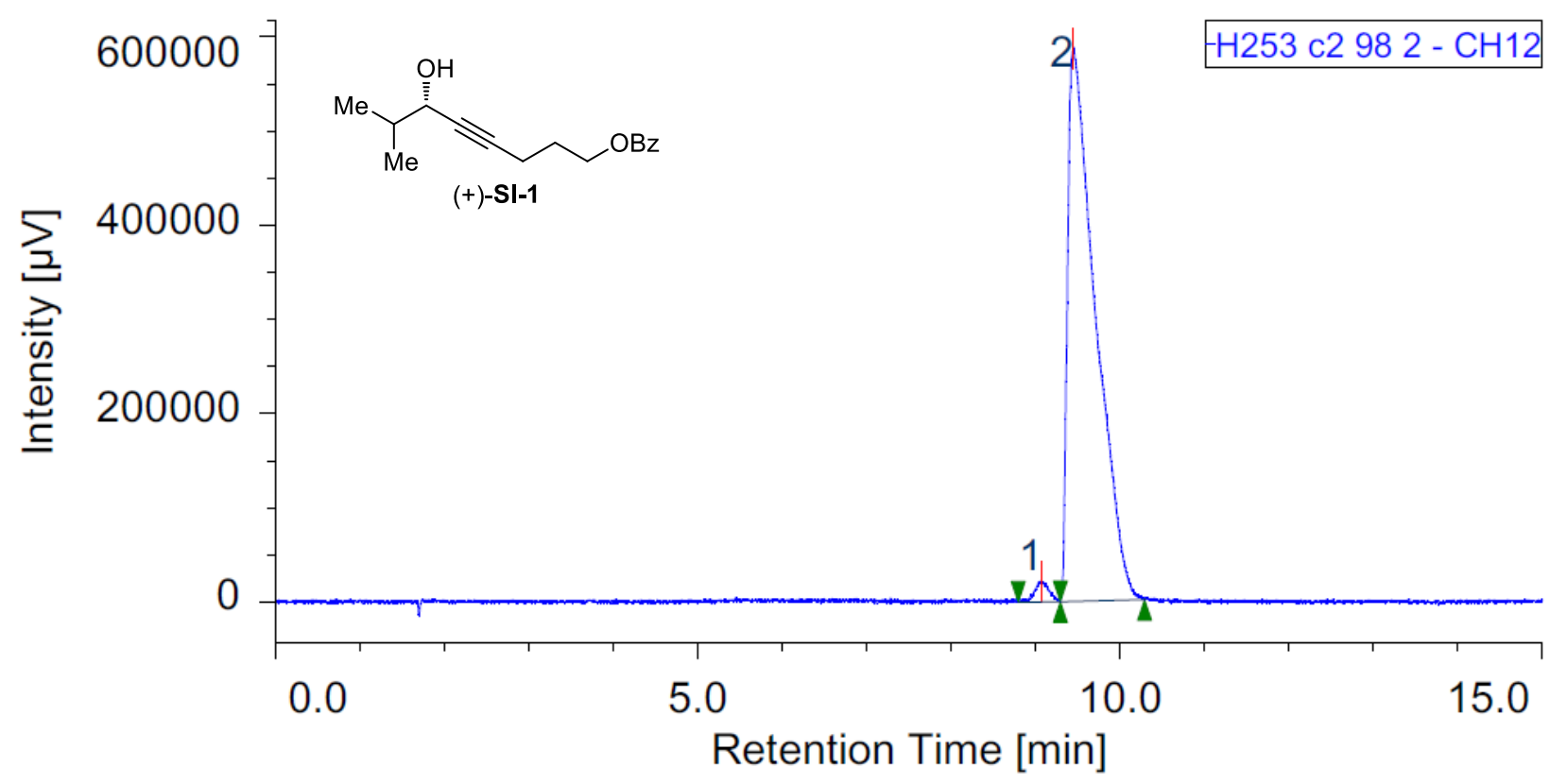

\begin{tabular}{|r|c|r|r|r|r|r|r|r|r|r|r|}
\hline$\#$ & Peak Name & $\mathrm{CH}$ & \multicolumn{1}{c}{$\mathrm{tR}[\mathrm{min}]$} & \multicolumn{1}{|c|}{ Area $[\mu \mathrm{V}-\mathrm{sec}]$} & Height $[\mu \mathrm{V}]$ & Area $\%$ & Height $\%$ & Quantity & NTP & Resolution & Symmetry Factor \\
\hline 1 & Unknown & 12 & 9.073 & 267298 & 2173 & 1.963 & 3.572 & N/A & 13248 & 0.832 & 0.893 \\
\hline 2 & Unknown & 12 & 9.447 & 13349165 & 586821 & 98.037 & 96.428 & N/A & 4171 & N/A & \\
\hline
\end{tabular}




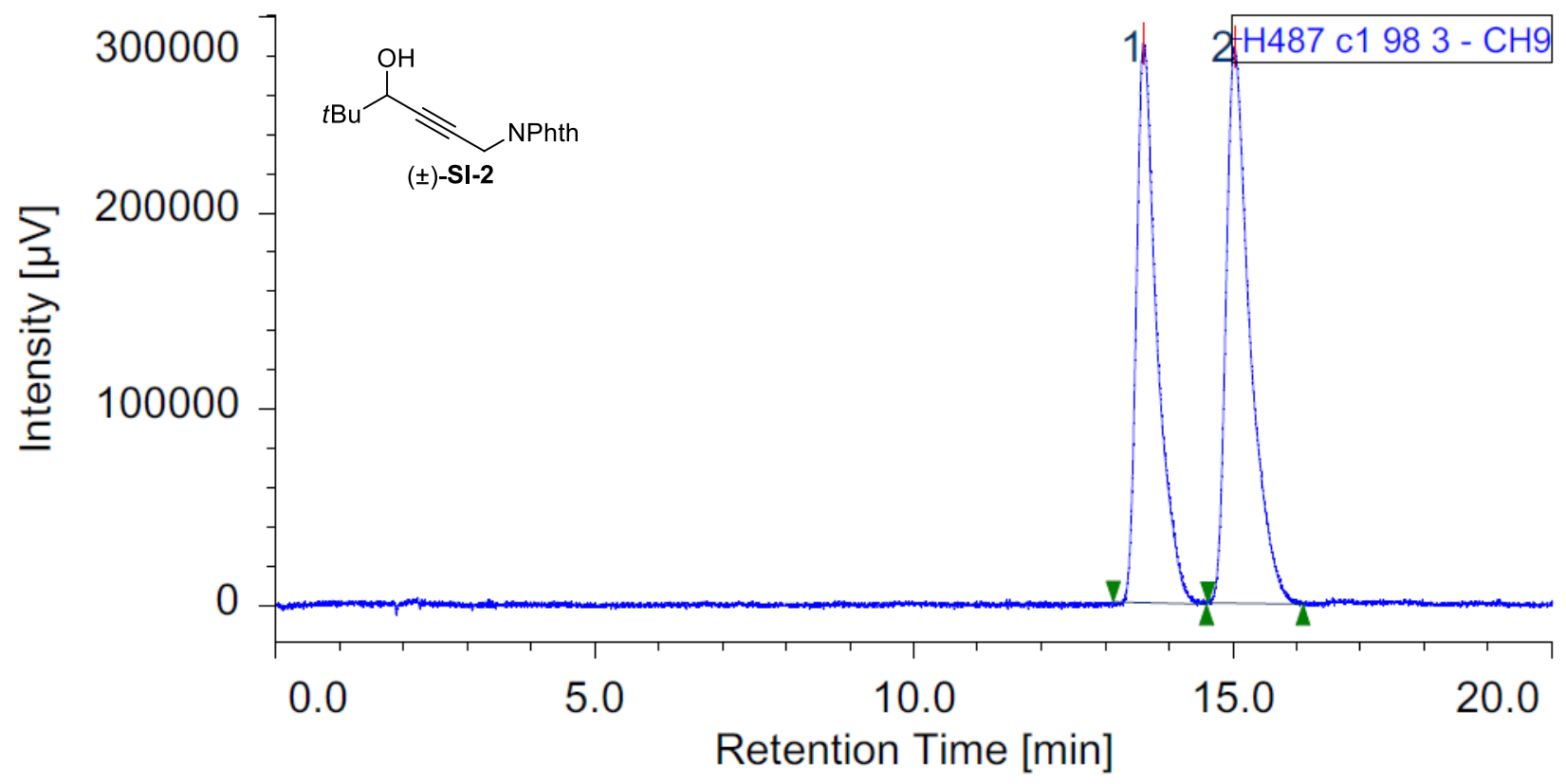

\begin{tabular}{|r|c|c|r|r|r|r|r|r|r|r|r|}
\hline$\#$ & Peak Name & $\mathrm{CH}$ & $\mathrm{tR}[\mathrm{min}]$ & Area $[\mu \mathrm{V}$-sec] & Height $[\mu \mathrm{V}]$ & Area $\%$ & Height $\%$ & Quantity & NTP & Resolution & Symmetry Factor \\
\hline 1 & Unknown & 9 & 13.593 & 6467741 & 284591 & 46.765 & 50.159 & N/A & 9517 & 2.396 & 1.736 \\
\hline 2 & Unknown & 9 & 15.027 & 7362670 & 282788 & 53.23 & 49.841 & N/A & 8759 & N/A & 1.723 \\
\hline
\end{tabular}

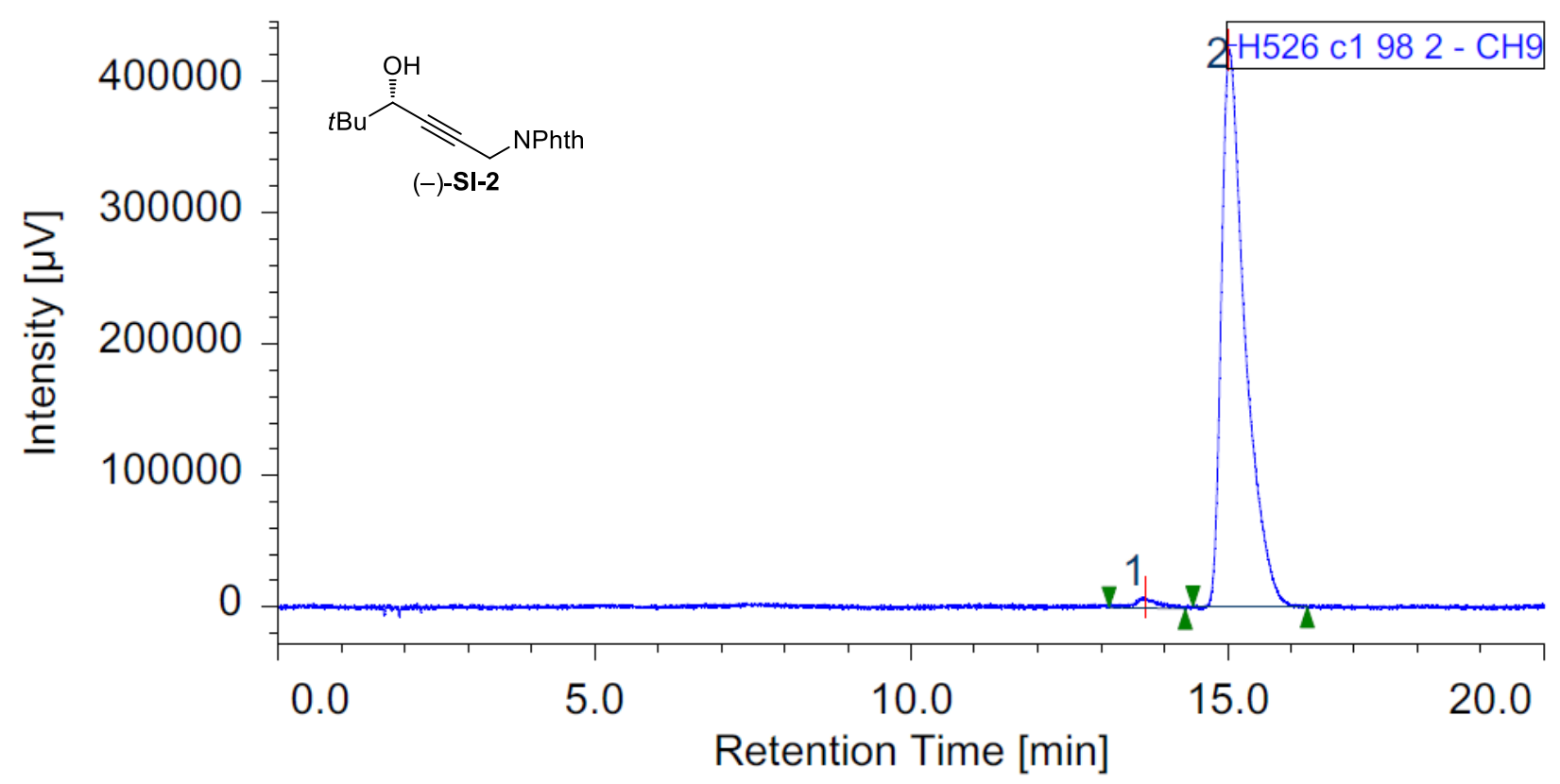

\begin{tabular}{|r|c|c|r|r|r|r|r|r|r|r|r|}
\hline$\#$ & Peak Name & $\mathrm{CH}$ & $\mathrm{tR}[\mathrm{min}]$ & Area $[\mu \mathrm{V} \cdot \mathrm{sec}]$ & Height $[\mu \mathrm{V}]$ & Area $\%$ & Height $\%$ & Quantity & NTP & Resolution & Symmetry Factor \\
\hline 1 & Unknown & 9 & 13.687 & 21388 & 8441 & 1.900 & 1.954 & N/A & 6196 & 1.999 & 1.065 \\
\hline 2 & Unknown & 9 & 15.020 & 11045085 & 423586 & 98.100 & 98.046 & N/A & 8748 & N/A & \\
\hline
\end{tabular}




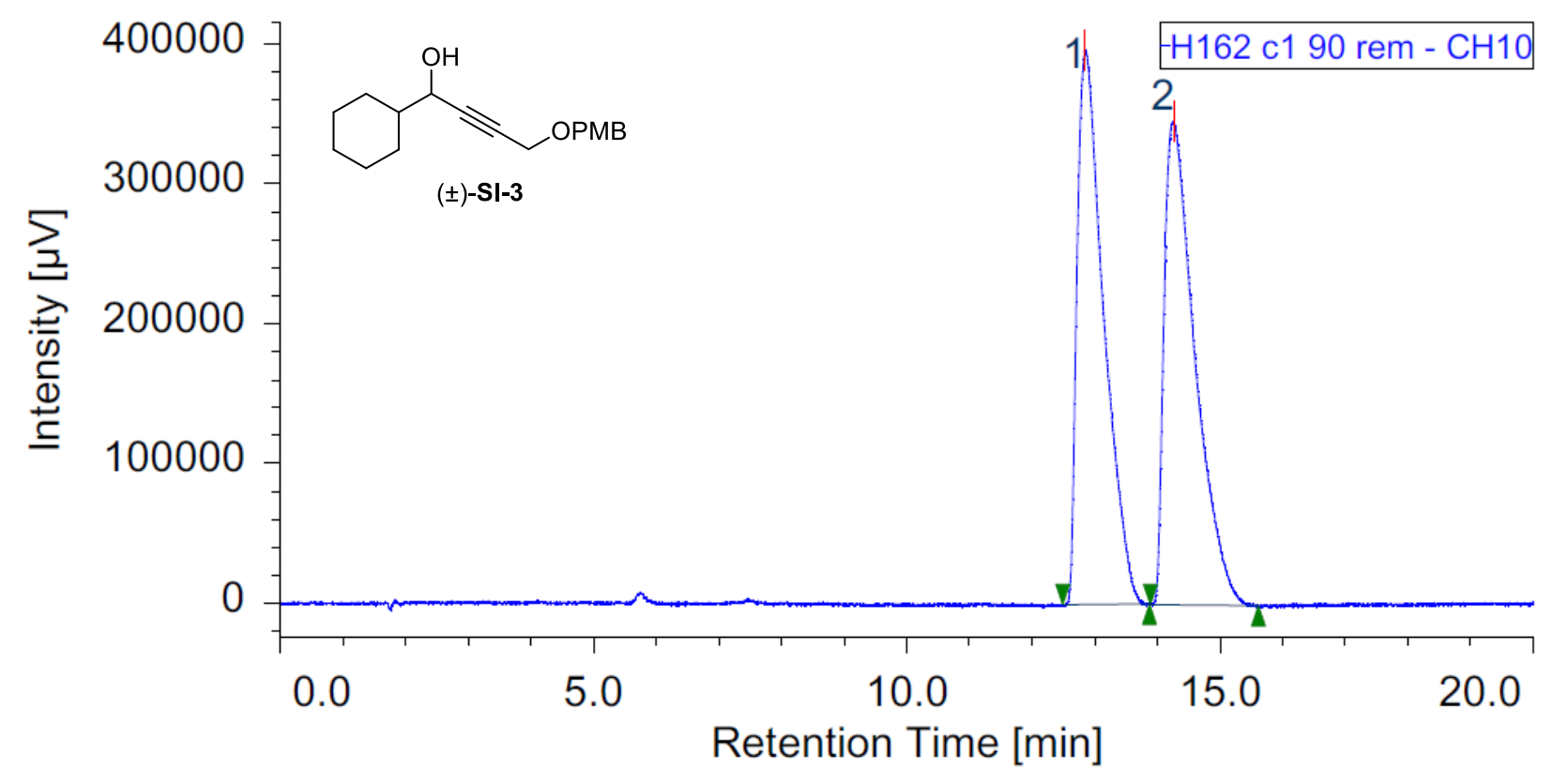

\begin{tabular}{|c|c|c|c|c|c|c|c|c|c|c|c|}
\hline$\#$ & Peak Name & $\mathrm{CH}$ & $2[\mathrm{~min}]$ & Area $[\mu \mathrm{V} \cdot \sec ]$ & [eight $[\mu \mathrm{V}]$ & Area $\%$ & Height $\%$ & Quantity & NTP & Resolution & y Factor \\
\hline & Unknown & 10 & 12.847 & 11658589 & 396001 & 49.731 & 53.416 & N/A & 4431 & 1.709 & 2.042 \\
\hline & Unknown & 10 & 14.260 & 11784701 & 345357 & 50.269 & 46.584 & $\mathrm{~N} / \mathrm{A}$ & 4141 & $\mathrm{~N} / \mathrm{A}$ & 2.160 \\
\hline
\end{tabular}

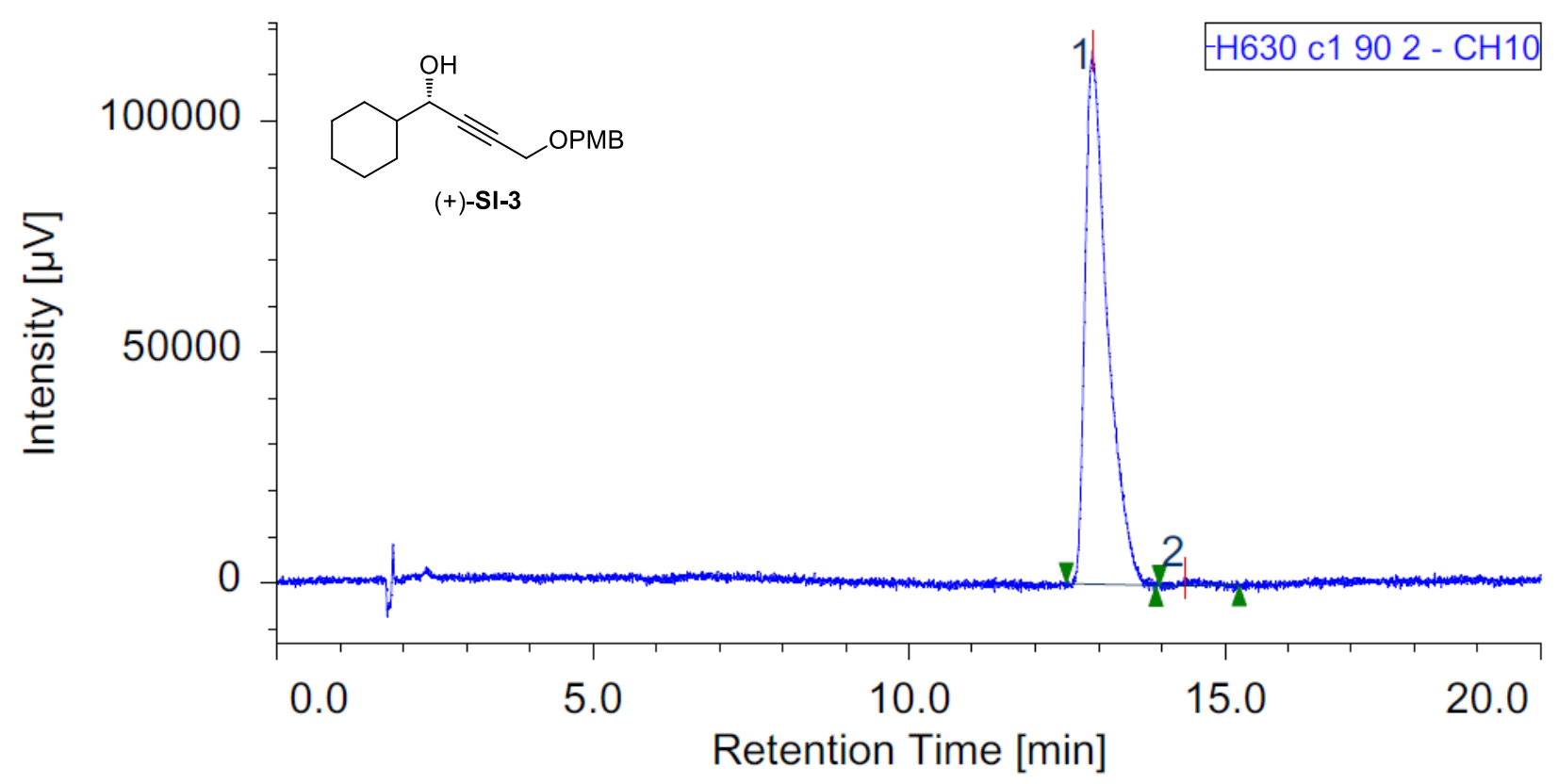

\begin{tabular}{|r|c|r|r|r|r|r|r|r|r|r|r|}
\hline$\#$ & Peak Name & $\mathrm{CH}$ & $\mathrm{tR}[\mathrm{min}]$ & Area $[\mu \mathrm{V} \cdot \mathrm{sec}]$ & Height $[\mu \mathrm{V}]$ & Area\% & Height $\%$ & Quantity & NTP & Resolution & Symmetry Factor \\
\hline 1 & Unknown & 10 & 12.900 & 2829767 & 115374 & 98.984 & 98.47 & N/A & 7308 & 1.676 & 1.865 \\
\hline 2 Unknown & 10 & 14.353 & 29056 & 1784 & 1.016 & 1.523 & N/A & 2559 & N/A & 1.592 \\
\hline
\end{tabular}




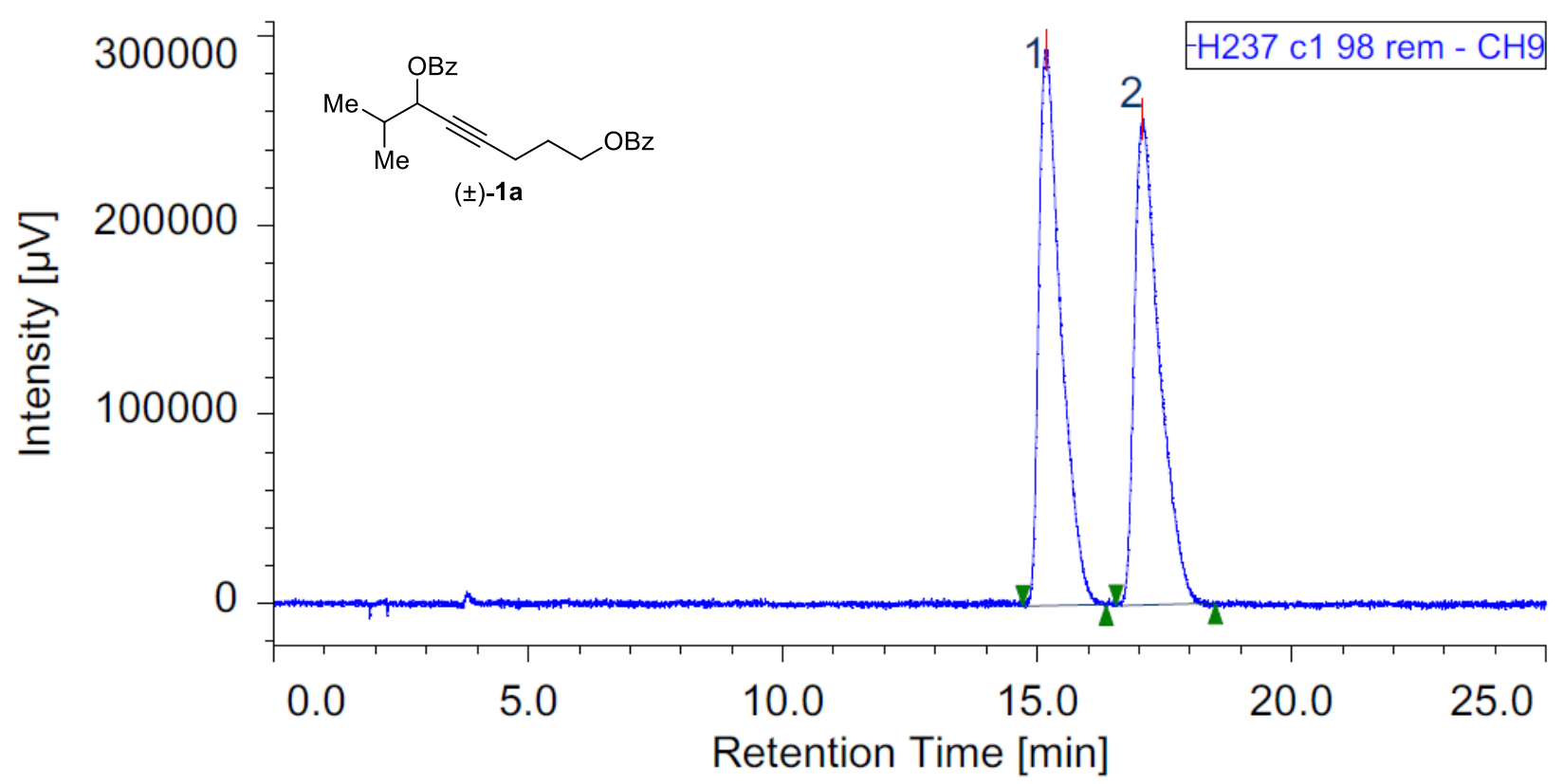

\begin{tabular}{|c|c|c|c|c|c|c|c|c|c|c|c|}
\hline \# & Peak Name & $\mathrm{CH}$ & $\mathrm{tR}$ [min] & Area $[\mu \mathrm{V} \cdot \mathrm{sec}]$ & Height $[\mu \mathrm{V}]$ & Area $\%$ & Height $\%$ & Quantity & NTP & Resolution & Factor \\
\hline & Unknown & 9 & 15.167 & 8882478 & 293888 & 50.120 & 53.362 & N/A & 6144 & 2.307 & 1.893 \\
\hline & Unknown & 9 & 17.067 & 8840032 & 256857 & 49.880 & 46.638 & N/A & 6052 & N/A & 1.857 \\
\hline
\end{tabular}

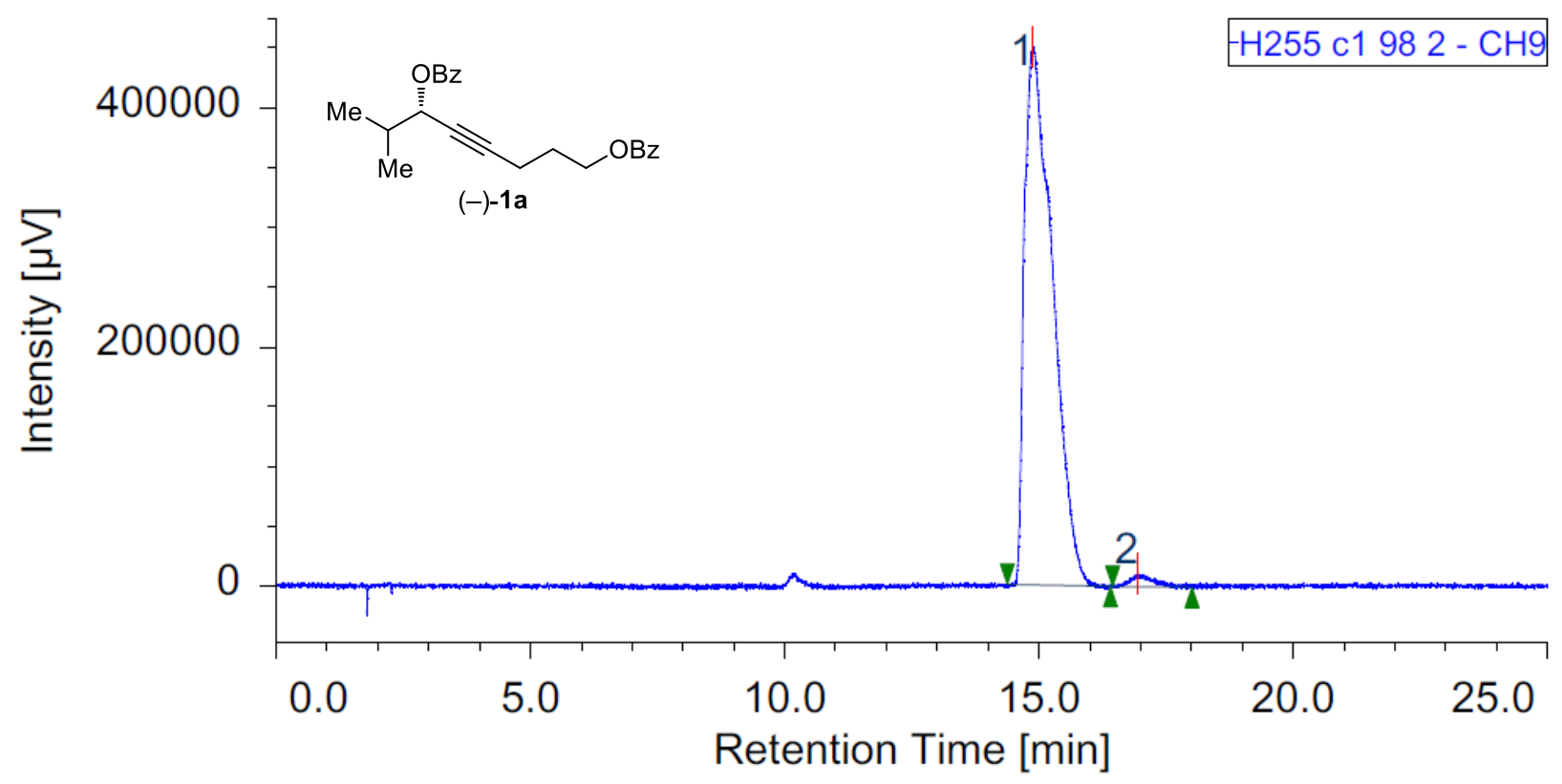

\begin{tabular}{|r|c|c|r|r|r|r|r|r|r|r|r|}
\hline$\#$ & Peak Name & CH & tR [min] & Area $[\mu \mathrm{V} \cdot \mathrm{sec}]$ & Height $[\mu \mathrm{V}]$ & Area $\%$ & Height $\%$ & Quantity & NTP & Resolution & Symmetry Factor \\
\hline 1 & Unknown & 9 & 14.880 & 17270165 & 449445 & 98.083 & 97.66 & N/A & 2954 & 2.112 & 2.031 \\
\hline 2 & Unknown & 9 & 16.940 & 337611 & 10743 & 1.917 & 2.334 & N/A & 6198 & N/A & \\
\hline
\end{tabular}




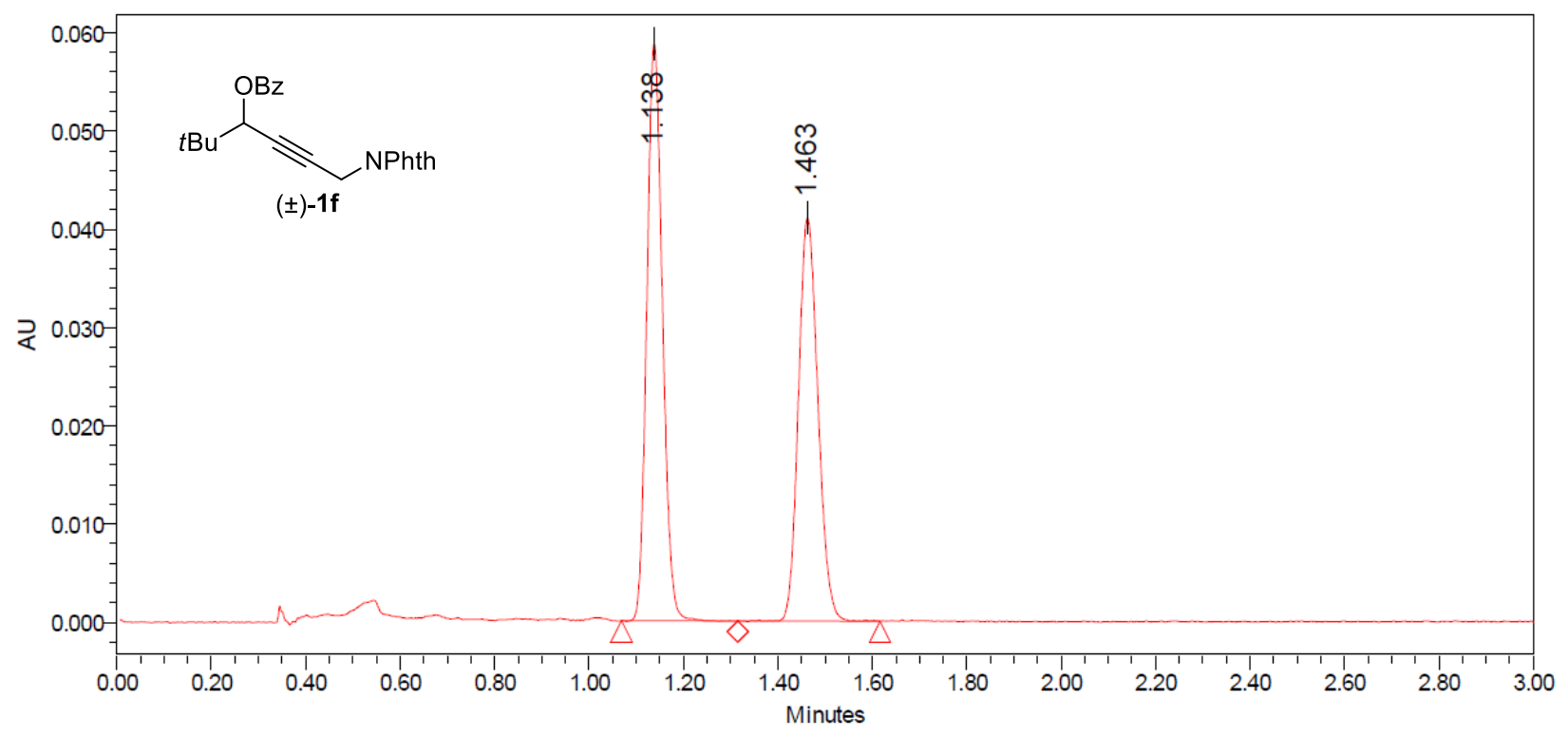

\begin{tabular}{|c|c|r|r|r|}
\hline & Processed Channel & $\begin{array}{r}\text { Retention } \\
\text { Time (min) }\end{array}$ & Area & $\%$ Area \\
\hline 1 & PDA Ch1 254nm@1.2nm -Compens. & 1.138 & 135718 & 53.42 \\
\hline 2 & PDA Ch1 254nm@1.2nm -Compens. & 1.463 & 118364 & 46.58 \\
\hline
\end{tabular}

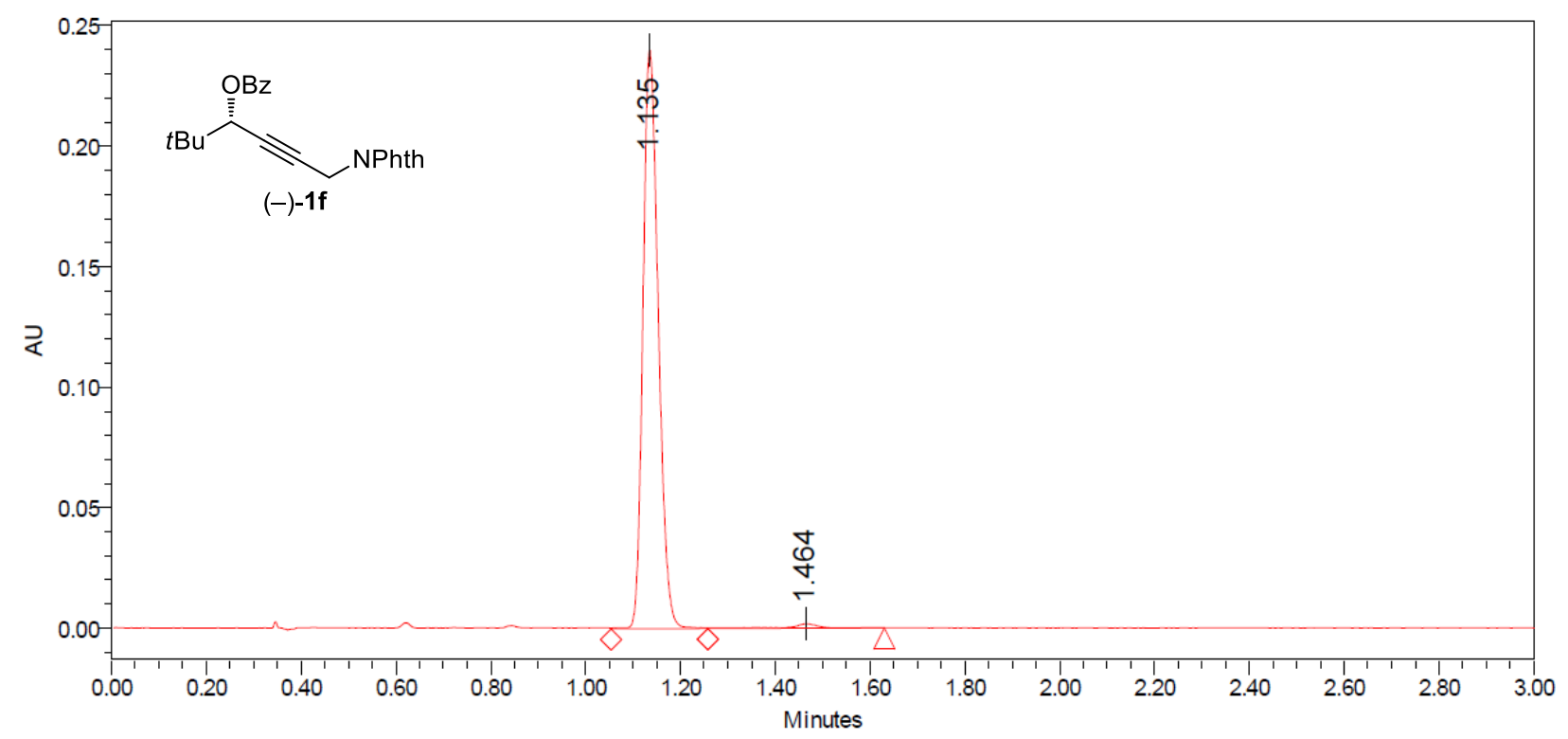

\begin{tabular}{|c|c|r|r|r|}
\hline & Processed Channel & $\begin{array}{r}\text { Retention } \\
\text { Time (min) }\end{array}$ & Area & $\%$ Area \\
\hline 1 & PDA Ch1 254nm@1.2nm -Compens. & 1.135 & 541841 & 98.40 \\
\hline 2 & PDA Ch1 254nm@1.2nm -Compens. & 1.464 & 8823 & 1.60 \\
\hline
\end{tabular}




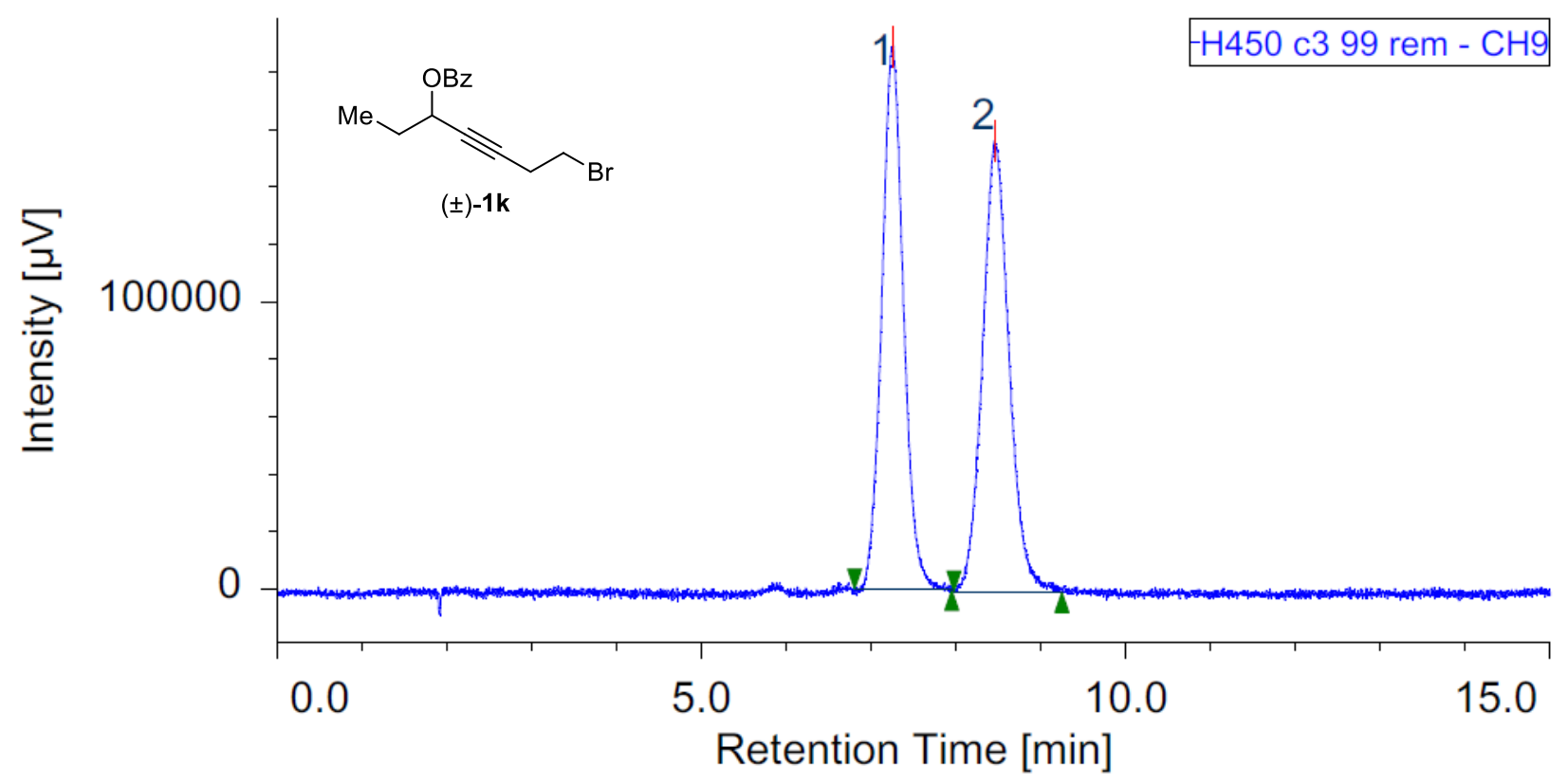

\begin{tabular}{|c|c|c|c|c|c|c|c|c|c|c|c|}
\hline$\#$ & Peak Name & $\mathrm{CH}$ & $\mathrm{tR}$ [min] & Area $[\mu \mathrm{V}$-sec] & Height $[\mu \mathrm{V}]$ & Area $\%$ & Height $\%$ & Quantity & NTP & Resolution & Symmetry Factor \\
\hline & Unknown & 9 & 7.260 & 3360009 & 188947 & 49.562 & 54.663 & N/A & 4117 & 2.378 & 1.055 \\
\hline & Unknown & 9 & 8.453 & 3419425 & 156711 & 50.438 & 45.337 & N/A & 3727 & N/A & 1.115 \\
\hline
\end{tabular}

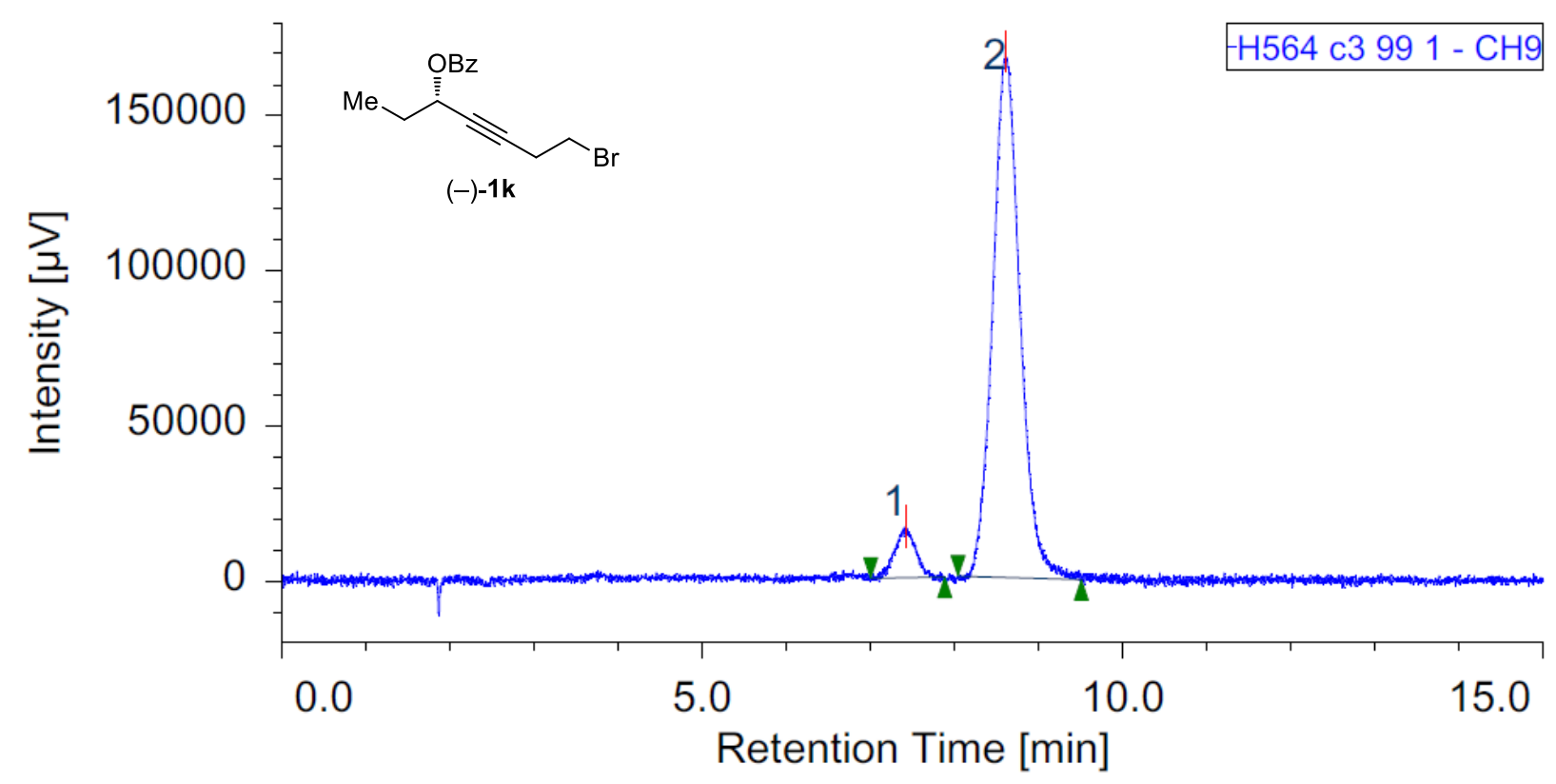

\begin{tabular}{|c|c|c|c|c|c|c|c|c|c|c|c|}
\hline$\#$ & Peak Name & $\mathrm{CH}$ & $\mathrm{tR}[\mathrm{min}]$ & Area $[\mu \mathrm{V} \cdot \mathrm{sec}]$ & Height $[\mu \mathrm{V}]$ & Area $\%$ & Height $\%$ & Quantity & NTP & Resolution & Symmetry Factor \\
\hline & Unknown & 9 & 7.420 & 281140 & 16573 & 6.914 & 8.901 & N/A & 4294 & 2.337 & 1.050 \\
\hline & Unknown & 9 & 8.607 & 3785265 & 169619 & 93.086 & 91.099 & N/A & 3708 & N/A & 1.130 \\
\hline
\end{tabular}

$$
\text { S-61 }
$$




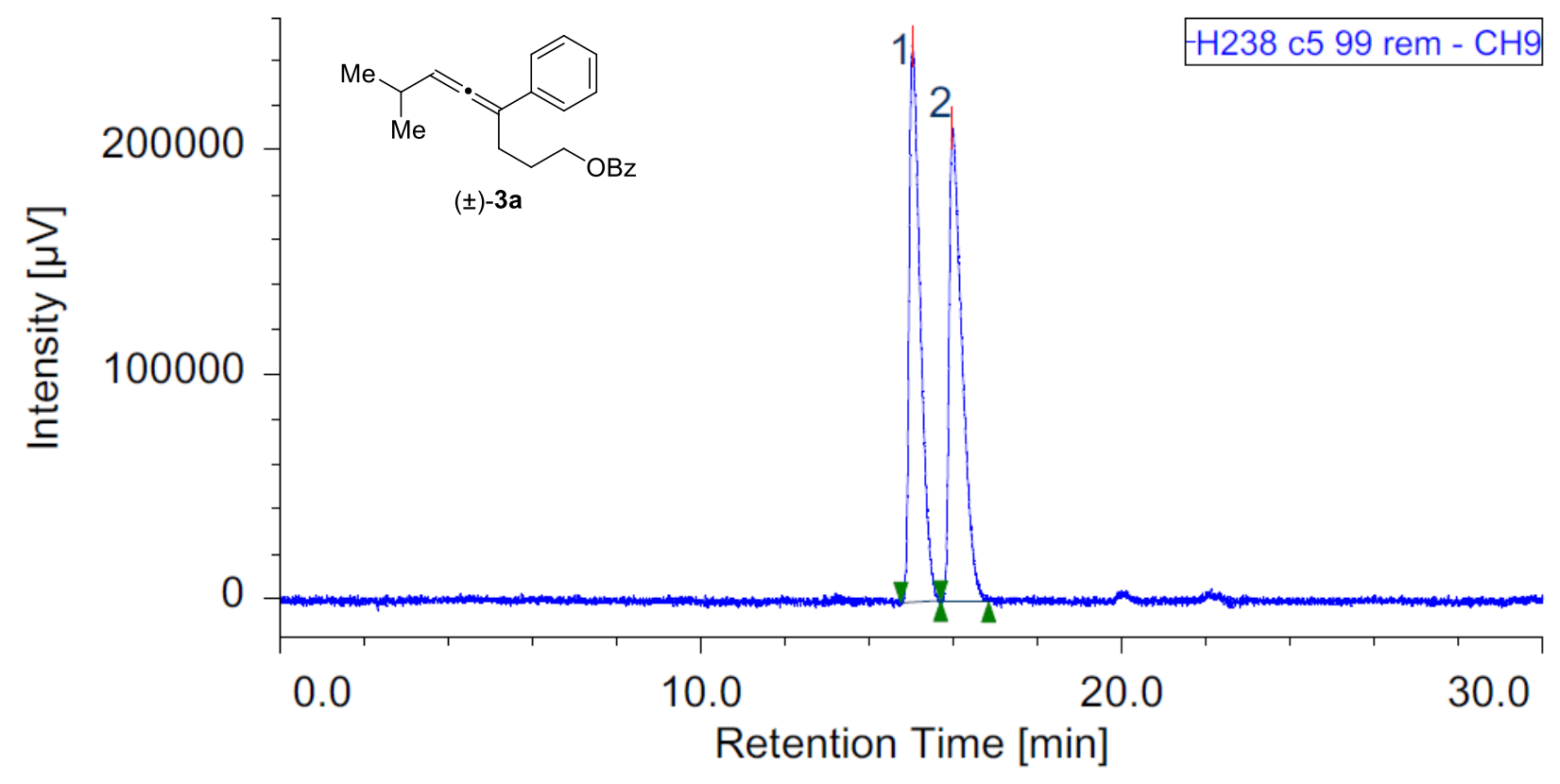

\begin{tabular}{|c|c|c|c|c|c|c|c|c|c|c|c|}
\hline \# & Peak Name & $\mathrm{CH}$ & $\mathrm{tR}$ [min] & Area $[\mu \mathrm{V}$-sec $]$ & Height $[\mu \mathrm{V}]$ & Area $\%$ & Height $\%$ & Quantity & NTP & Resolution & Symmetry Factor \\
\hline & Unknown & 9 & 15.020 & 4518708 & 247615 & 49.925 & 54.023 & N/A & 15827 & 1.838 & 1.978 \\
\hline & Unknown & 9 & 15.967 & 4532315 & 210740 & 50.075 & 45.977 & N/A & 13217 & $\mathrm{~N} / \mathrm{A}$ & 2.320 \\
\hline
\end{tabular}

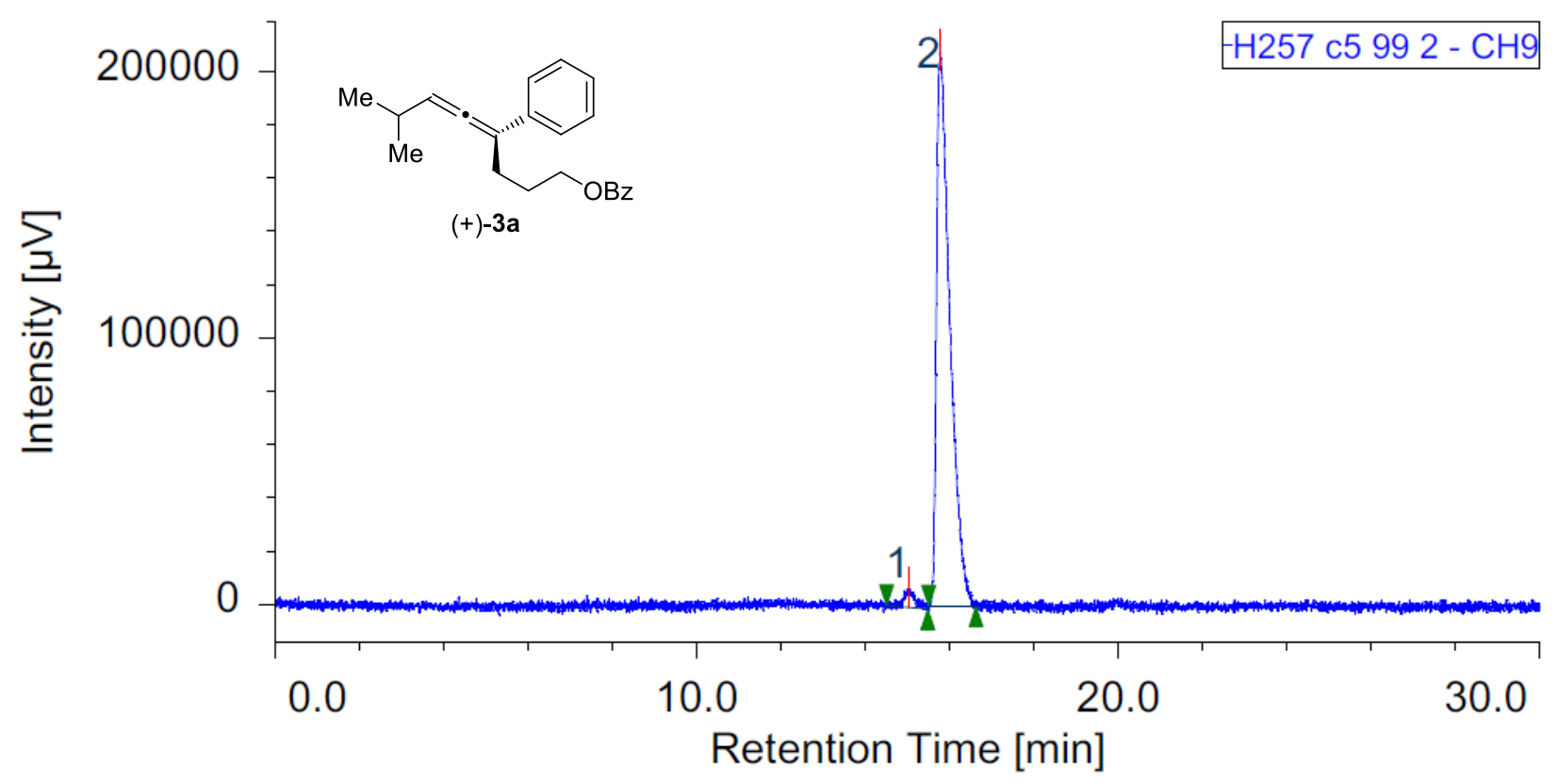

\begin{tabular}{|r|c|c|r|r|r|r|r|r|r|r|r|}
\hline$\#$ & Peak Name & $\mathrm{CH}$ & \multicolumn{1}{|c}{$[\mathrm{min}]$} & Area $[\mu \mathrm{V} \cdot \mathrm{sec}]$ & Height $[\mu \mathrm{V}]$ & \multicolumn{1}{c|}{ Area\% } & Height $\%$ & Quantity & NTP & Resolution & Symmetry Factor \\
\hline 1 & Unknown & 9 & 15.027 & 132255 & 7218 & 2.816 & 3.339 & N/A & 17097 & 1.456 & 0.955 \\
\hline 2 Unknown & 9 & 15.767 & 4564425 & 208961 & 97.184 & 96.661 & N/A & 12692 & N/A & 2.385 \\
\hline
\end{tabular}




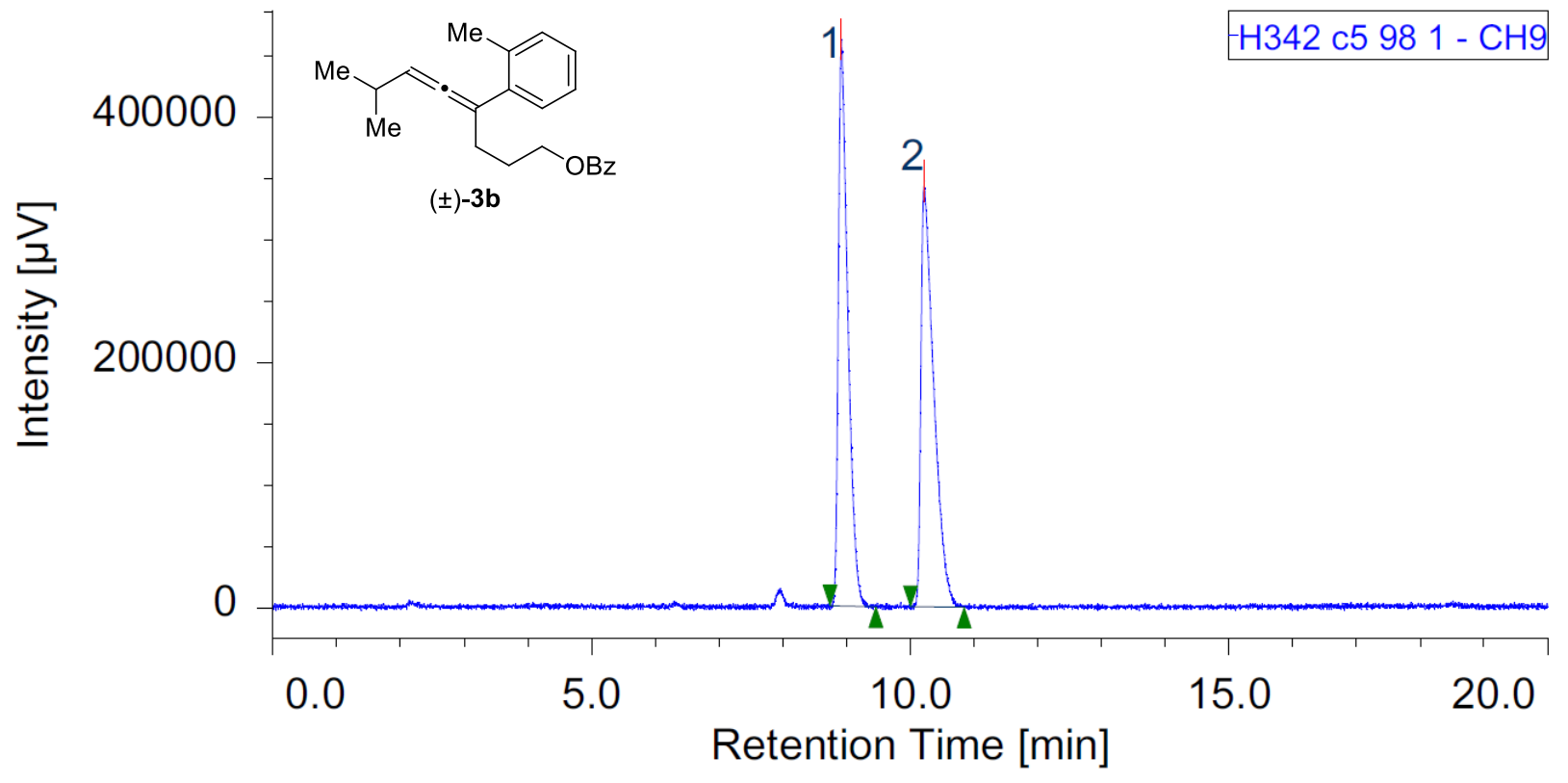

\begin{tabular}{|r|c|c|r|r|r|r|r|r|r|r|r|}
\hline$\#$ & Peak Name & $\mathrm{CH}$ & \multicolumn{1}{|c|}{ [R [min] } & Area $[\mu \mathrm{V} \cdot \mathrm{sec}]$ & Height $[\mu \mathrm{V}]$ & Area $\%$ & Height $\%$ & Quantity & NTP & Resolution & Symmetry Factor \\
\hline 1 & Unknown & 9 & 8.920 & 4851250 & 46215 & 50.026 & 57.110 & N/A & 17338 & 4.091 & 1.752 \\
\hline 2 & Unknown & 9 & 10.213 & 4846116 & 347089 & 49.974 & 42.890 & N/A & 12670 & N/A & \\
\hline
\end{tabular}

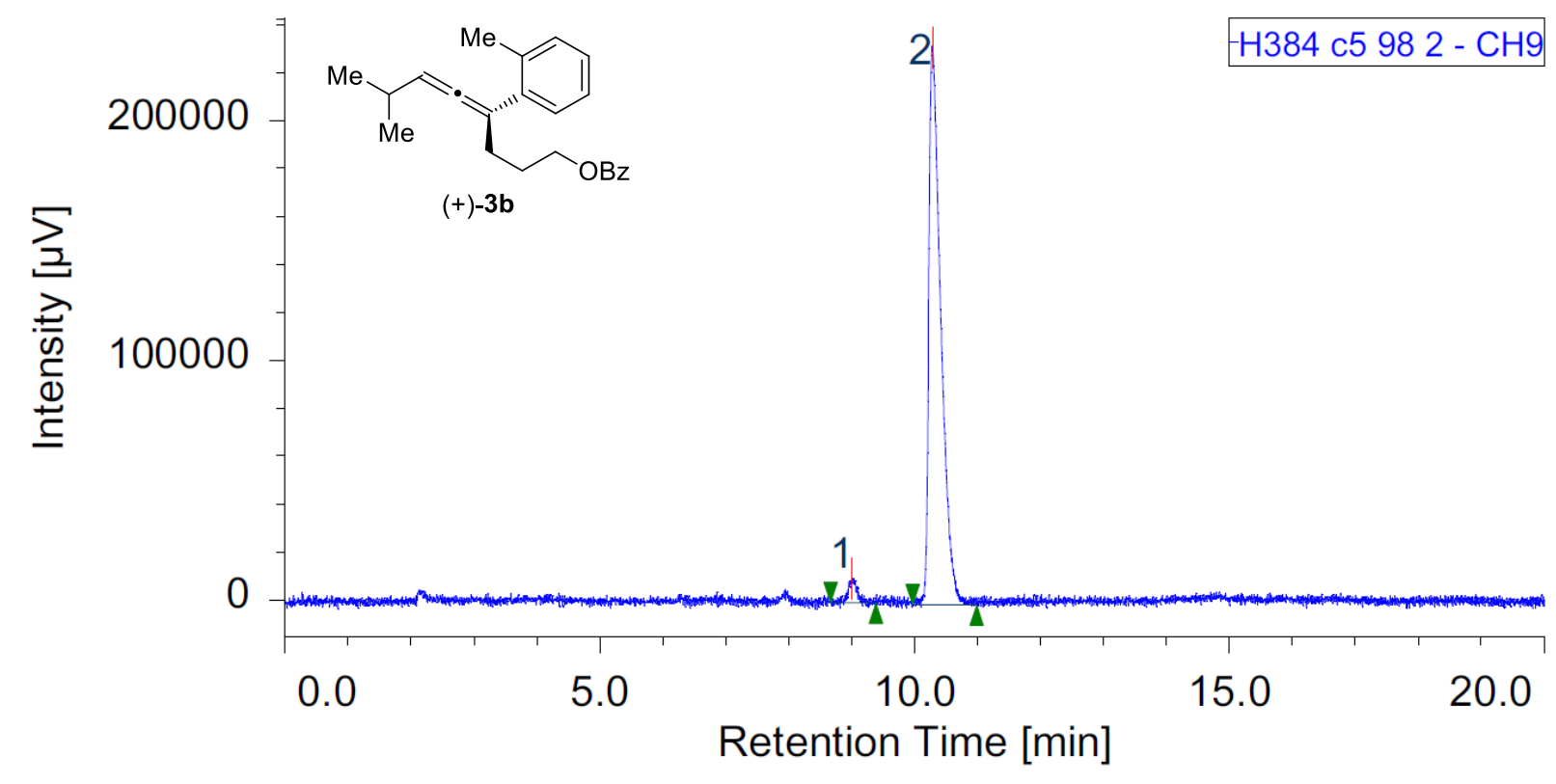

\begin{tabular}{|c|c|c|c|c|c|c|c|c|c|c|c|}
\hline \# & Peak Name & $\mathrm{CH}$ & $\mathrm{tR}$ [min] & Area $[\mu \mathrm{V} \cdot \mathrm{sec}]$ & Height $[\mu \mathrm{V}]$ & Area\% & Height $\%$ & Quantity & NTP & Resolution & Symmetry Factor \\
\hline & Unknown & 9 & 9.007 & 109464 & 10256 & 3.440 & 4.233 & N/A & 21281 & 4.341 & 1.061 \\
\hline & Unknown & 9 & 10.280 & 3072385 & 232048 & 96.560 & 95.767 & $\mathrm{~N} / \mathrm{A}$ & 14520 & N/A & 1.98 \\
\hline
\end{tabular}




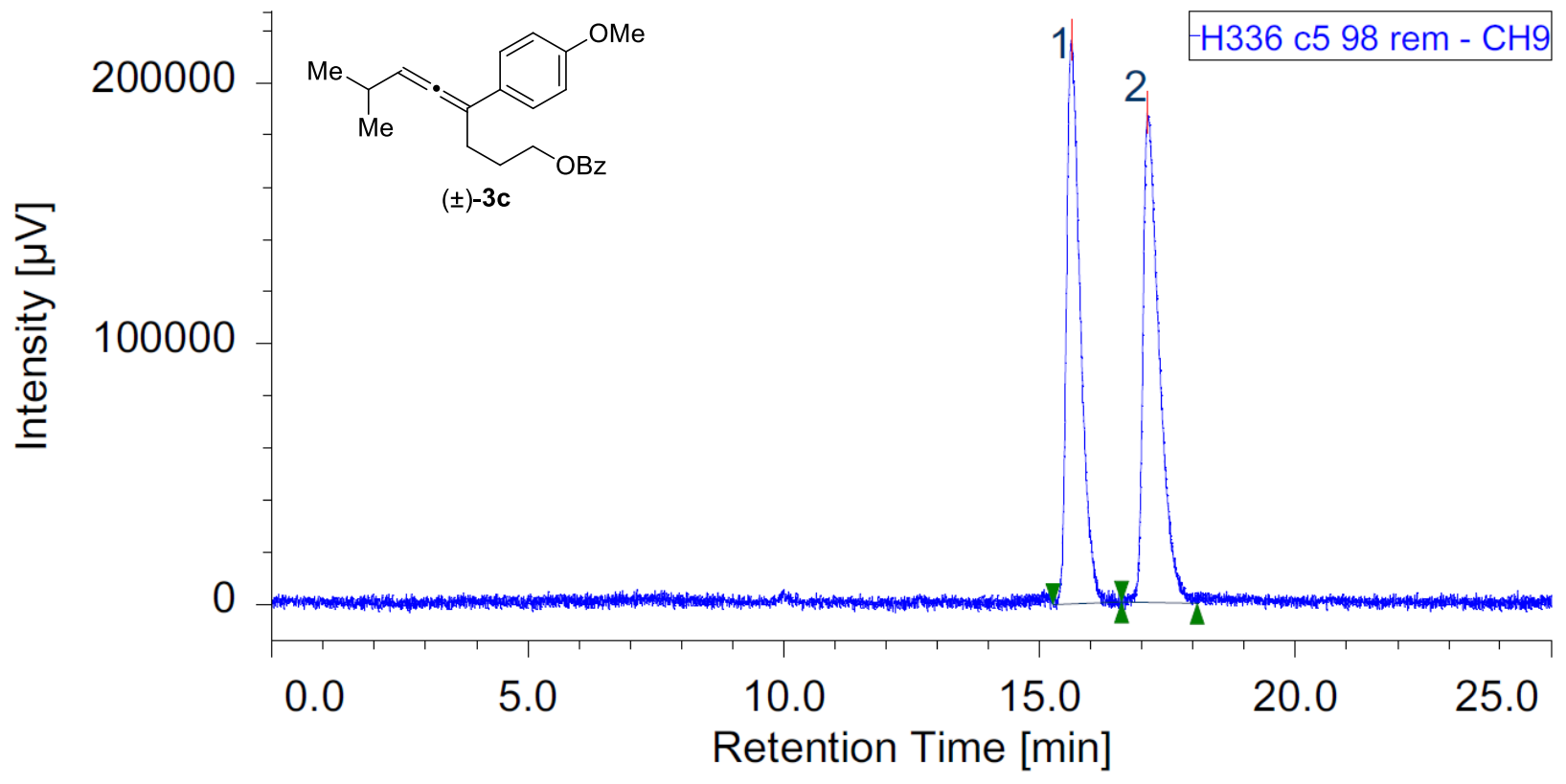

\begin{tabular}{|c|c|c|c|c|c|c|c|c|c|c|}
\hline \begin{tabular}{|l|l|}
$\#$ & Peak Name \\
\end{tabular} & $\mathrm{CH}$ & $\mathrm{tR}$ [min] & Area $[\mu \mathrm{V} \cdot \sec ]$ & Height $[\mu \mathrm{V}]$ & Area $\%$ & Height $\%$ & Quantity & NTP & Resolution & Symmetry Factor \\
\hline Unknown & 9 & 15.620 & 4079345 & 216174 & $49.48 \mathrm{C}$ & 53.523 & $\mathrm{~N} / \mathrm{A}$ & 1659 & 2.85 & 1.649 \\
\hline 2 Unknown & 9 & 17.100 & 4164109 & 187719 & 50.51 & 46.477 & $\mathrm{~N} / \mathrm{A}$ & 1511 & $\overline{\mathrm{N} / \mathrm{A}}$ & 2.179 \\
\hline
\end{tabular}

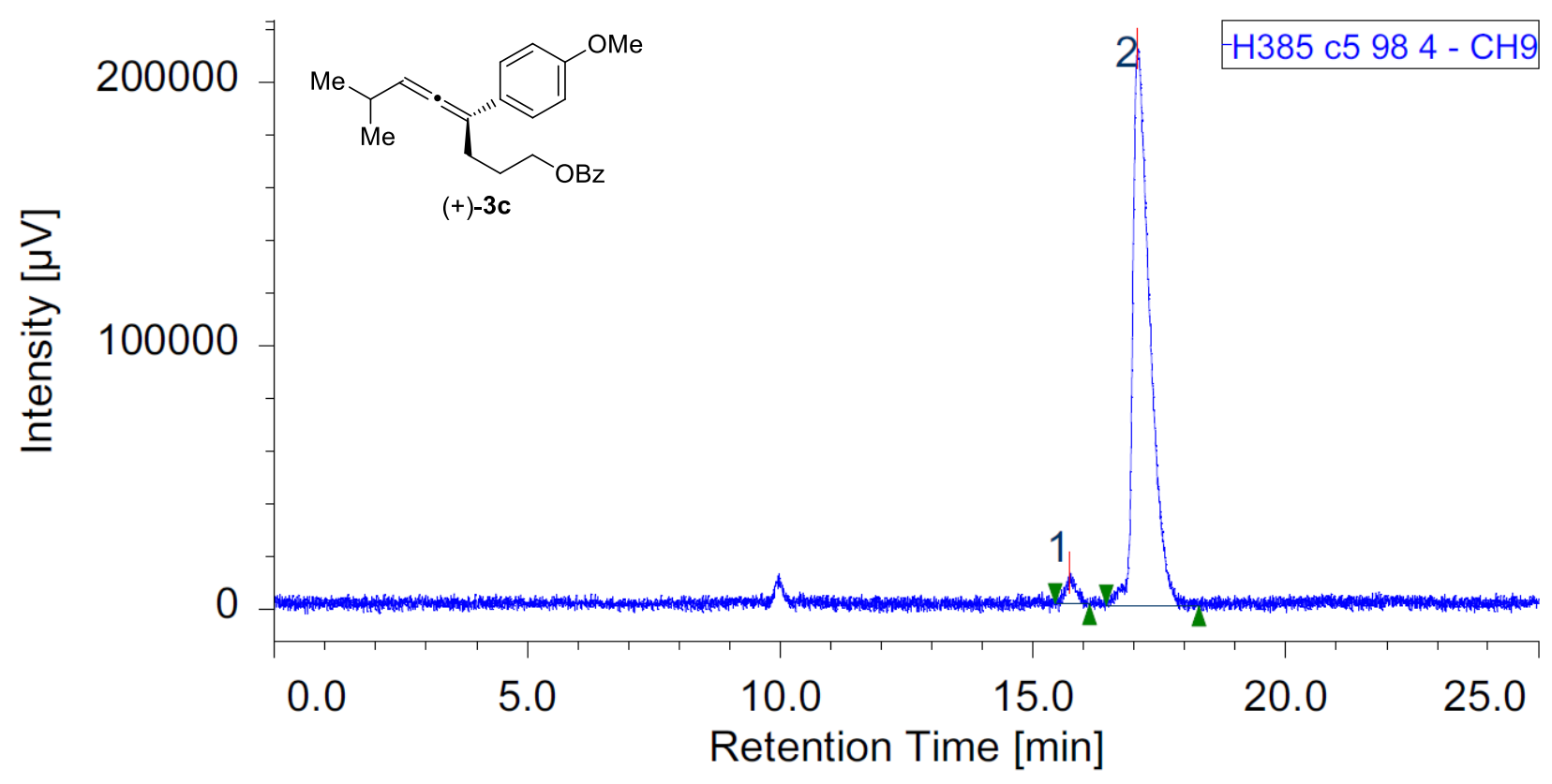

\begin{tabular}{|c|c|c|c|c|c|c|c|c|c|c|}
\hline \begin{tabular}{l|l}
$\#$ & Peak Name \\
\end{tabular} & $\mathrm{CH}$ & $\mathrm{tR}$ [min] & Area $[\mu \mathrm{V} \cdot \mathrm{sec}]$ & Height $[\mu \mathrm{V}]$ & Area\% $\%$ & Height $\%$ & Quantity & NTP & Resolution & Symmetry Factor \\
\hline Jnknown & 9 & 15.727 & 161148 & 11975 & 3.14 & 5.37 & $\mathrm{~N} / \mathrm{A}$ & 24235 & 2.684 & 1.103 \\
\hline Unknown & 9 & 17.067 & 4966245 & 210880 & 96.85 & 94.62 & $\mathrm{~N} / \mathrm{A}$ & 13074 & $\mathrm{~N} / \mathrm{A}$ & 2.011 \\
\hline
\end{tabular}




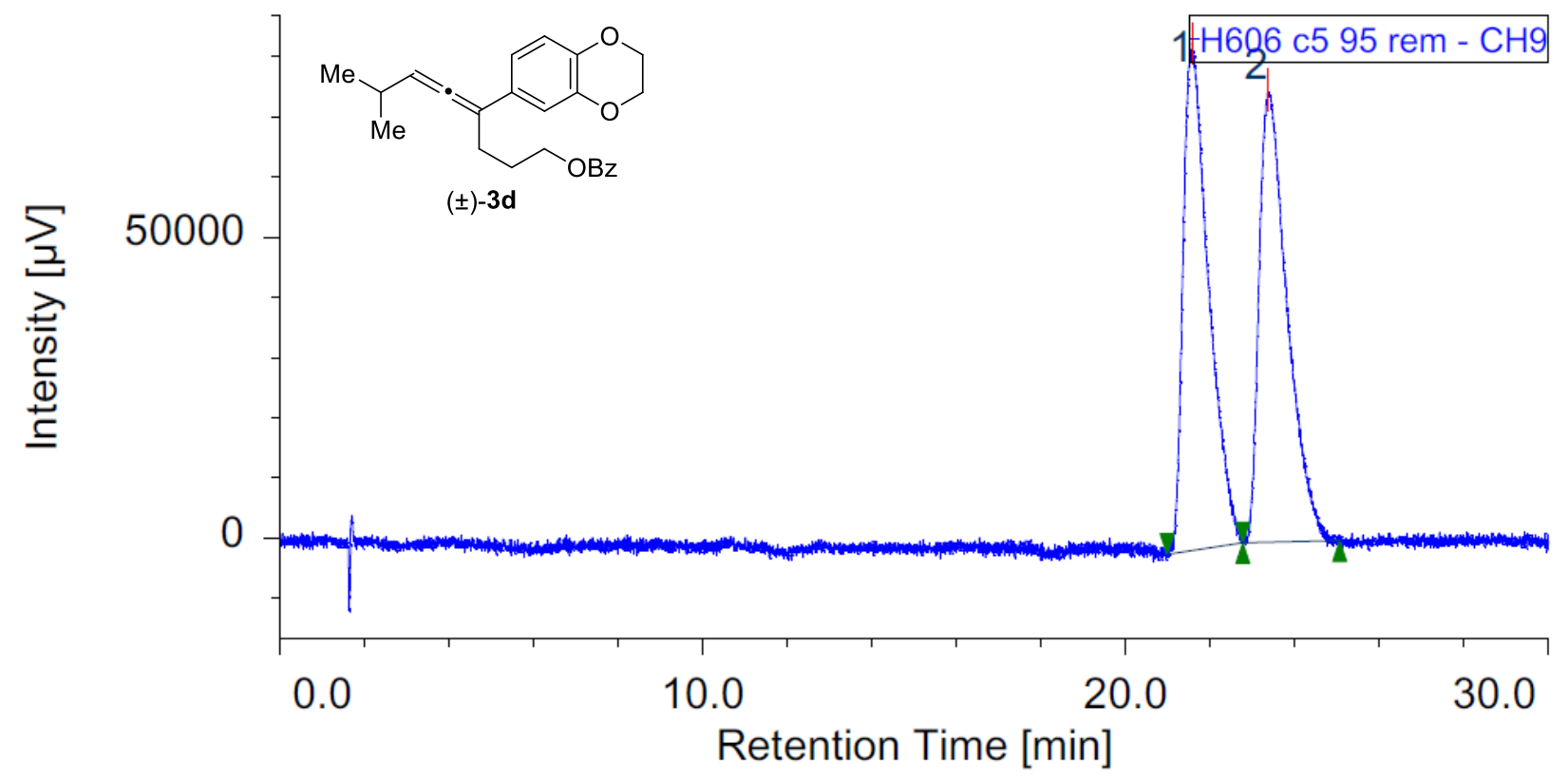

\begin{tabular}{|r|l|c|r|r|r|r|r|r|r|r|r|}
\hline$\#$ & Peak Name & $\mathrm{CH}$ & $\mathrm{tR}[\mathrm{min}]$ & Area $[\mu \mathrm{V} \cdot \mathrm{sec}]$ & Height $[\mu \mathrm{V}]$ & Area\% & Height $\%$ & Quantity & NTP & Resolution & Symmetry Factor \\
\hline 1 & Unknown & 9 & 21.573 & 3512786 & 84196 & 50.311 & 52.866 & N/A & 6327 & 1.562 & 1.760 \\
\hline 2 & Unknown & 9 & 23.360 & 3469375 & 75066 & 49.689 & 47.134 & N/A & 5970 & N/A & 1.923 \\
\hline
\end{tabular}

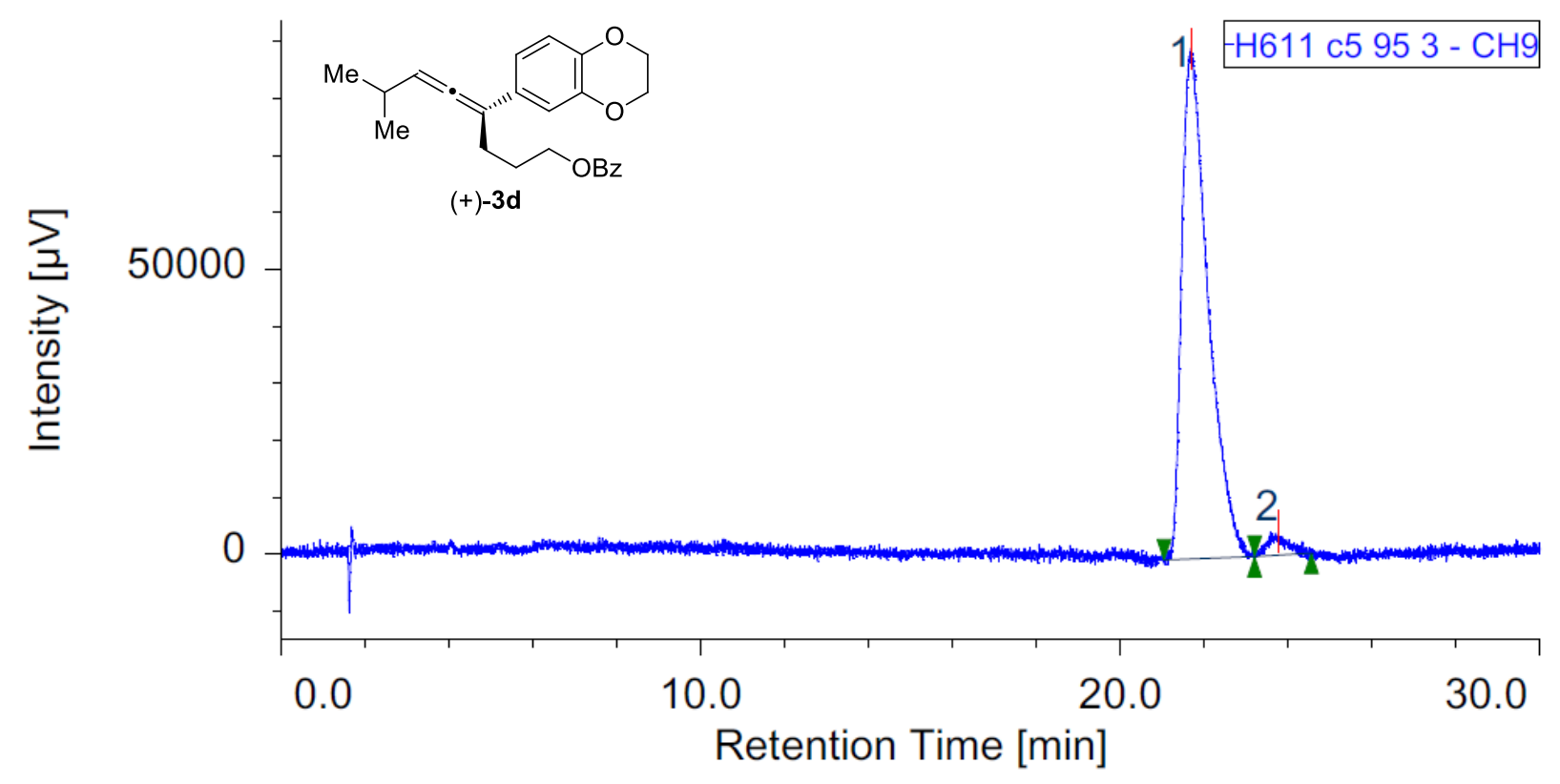

\begin{tabular}{|r|c|c|r|r|r|r|r|r|r|r|r|}
\hline$\#$ & Peak Name & $\mathrm{CH}$ & $\mathrm{tR}[\mathrm{min}]$ & Area $[\mu \mathrm{V}-\mathrm{sec}]$ & Height $[\mu \mathrm{V}]$ & Area $\%$ & Height $\%$ & Quantity & NTP & Resolution & Symmetry Factor \\
\hline 1 & Unknown & 9 & 21.680 & 388907 & 89703 & 96.833 & 95.551 & N/A & 6106 & 1.978 & 1.857 \\
\hline 2 & Unknown & 9 & 23.760 & 127212 & 417 & 3.167 & 4.449 & N/A & 9054 & N/A & \\
\hline
\end{tabular}




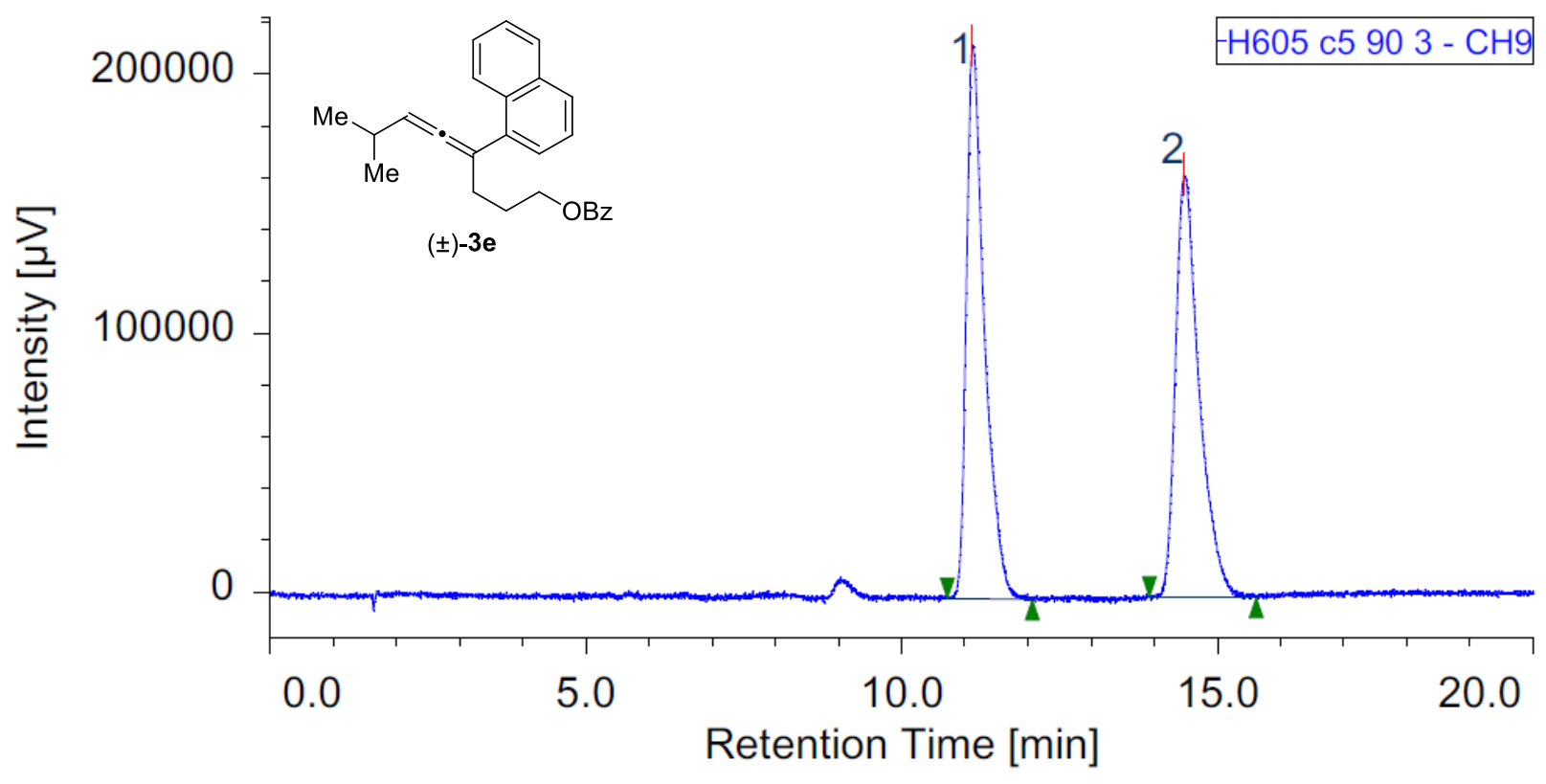

\begin{tabular}{|c|c|c|c|c|c|c|c|c|c|c|c|}
\hline$\#$ & Peak Name & $\mathrm{CH}$ & $\mathrm{tR}[\mathrm{min}]$ & Area $[\mu \mathrm{V}$-sec $]$ & Height $[\mu \mathrm{V}]$ & Area $\%$ & Height $\%$ & Quantity & NTP & Resolution & Symmetry Factor \\
\hline & Unknown & 9 & 11.120 & 4293038 & 213661 & 50.271 & 56.714 & N/A & 8151 & 5.871 & 1.680 \\
\hline 2 & Unknown & 9 & 14.460 & 4246701 & 163075 & 49.729 & 43.286 & N/A & 7962 & N/A & 1.610 \\
\hline
\end{tabular}

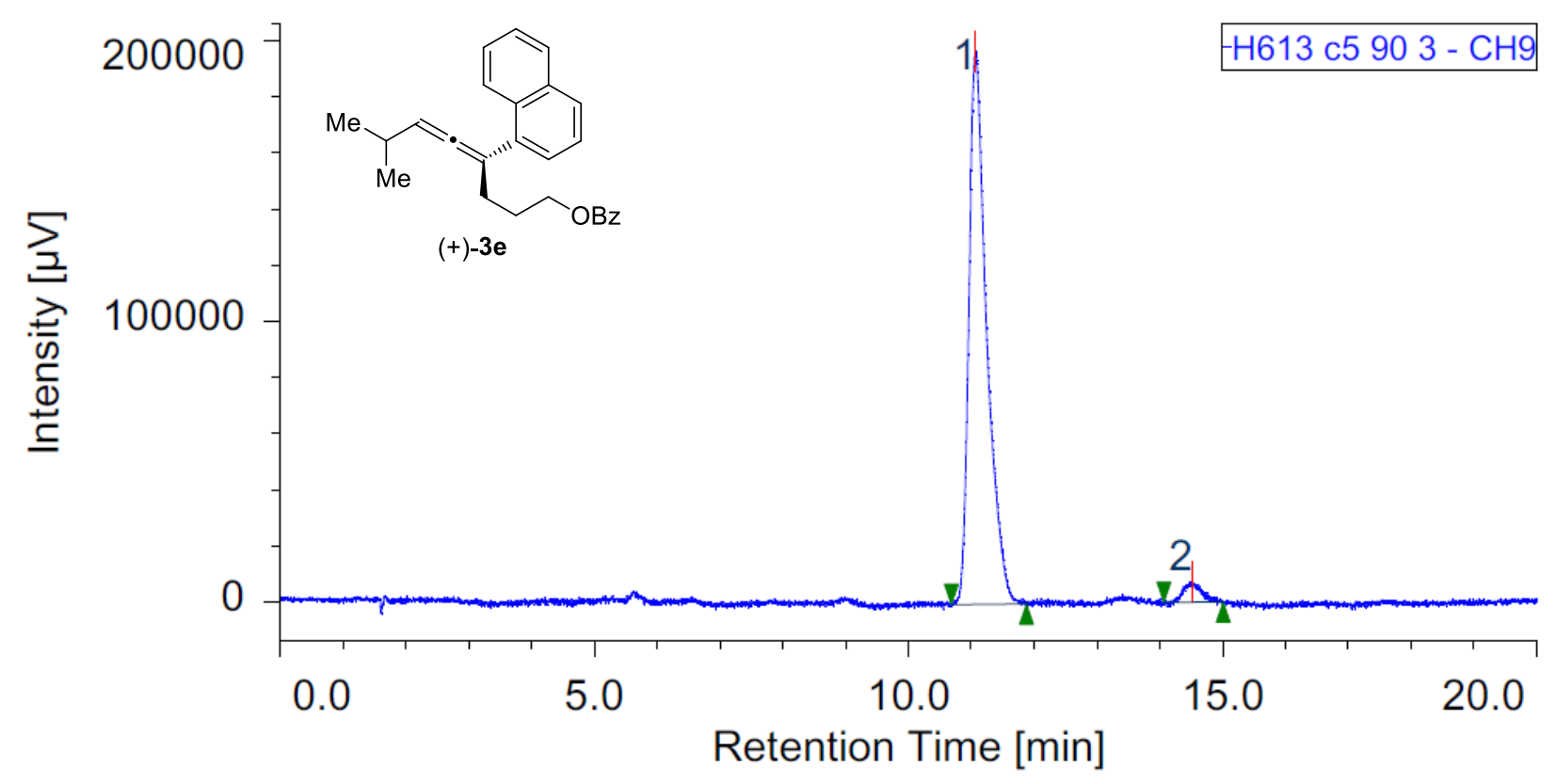

\begin{tabular}{|r|c|c|r|r|r|r|r|r|r|r|r|}
\hline$\#$ & Peak Name & $\mathrm{CH}$ & $\mathrm{tR}[\mathrm{min}]$ & Area $[\mu \mathrm{V} \cdot \mathrm{sec}]$ & Height $[\mu \mathrm{V}]$ & Area $\%$ & Height $\%$ & Quantity & NTP & Resolution & Symmetry Factor \\
\hline 1 & Unknown & 9 & 11.067 & 3931243 & 197010 & 96.532 & 96.447 & N/A & 7929 & 6.471 & 1.551 \\
\hline 2 Unknown & 9 & 14.507 & 141241 & 7257 & 3.468 & 3.553 & N/A & 10405 & N/A & 1.030 \\
\hline
\end{tabular}




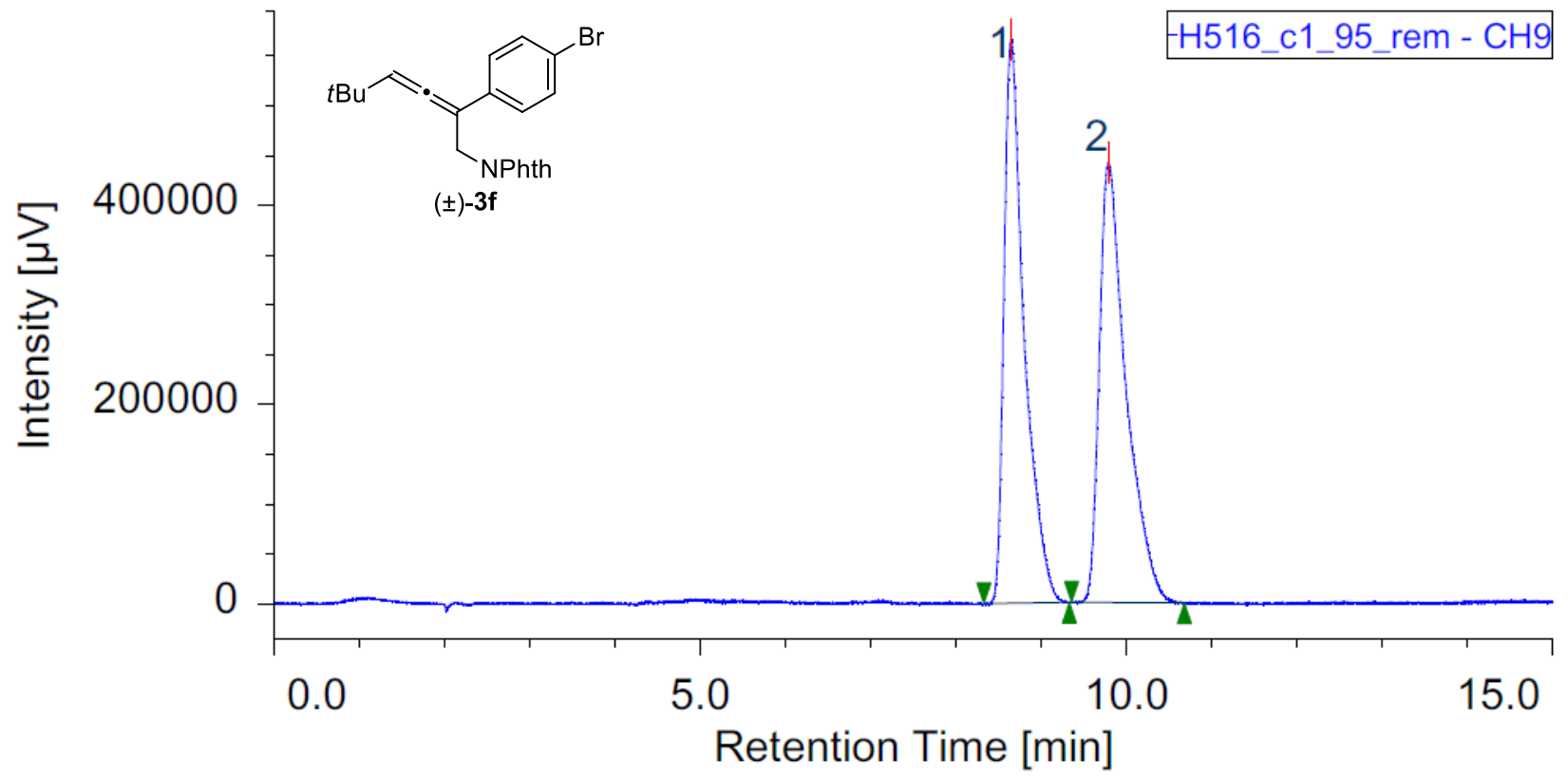

\begin{tabular}{|r|c|c|r|r|r|r|r|r|r|r|r|}
\hline$\#$ & Peak Name & $\mathrm{CH}$ & \multicolumn{1}{|c|}{$[\mathrm{min}]$} & Area $[\mu \mathrm{V} \cdot \mathrm{sec}]$ & Height $[\mu \mathrm{V}]$ & \multicolumn{1}{|c|}{ Area\% } & Height $\%$ & Quantity & NTP & Resolution & Symmetry Factor \\
\hline 1 & Unknown & 9 & 8.647 & 9303466 & 565794 & 49.226 & 56.209 & N/A & 7638 & 2.454 & 1.787 \\
\hline 2 & Unknown & 9 & 9.787 & 959589 & 440793 & 50.774 & 43.791 & N/A & 5335 & N/A & 1.770 \\
\hline
\end{tabular}

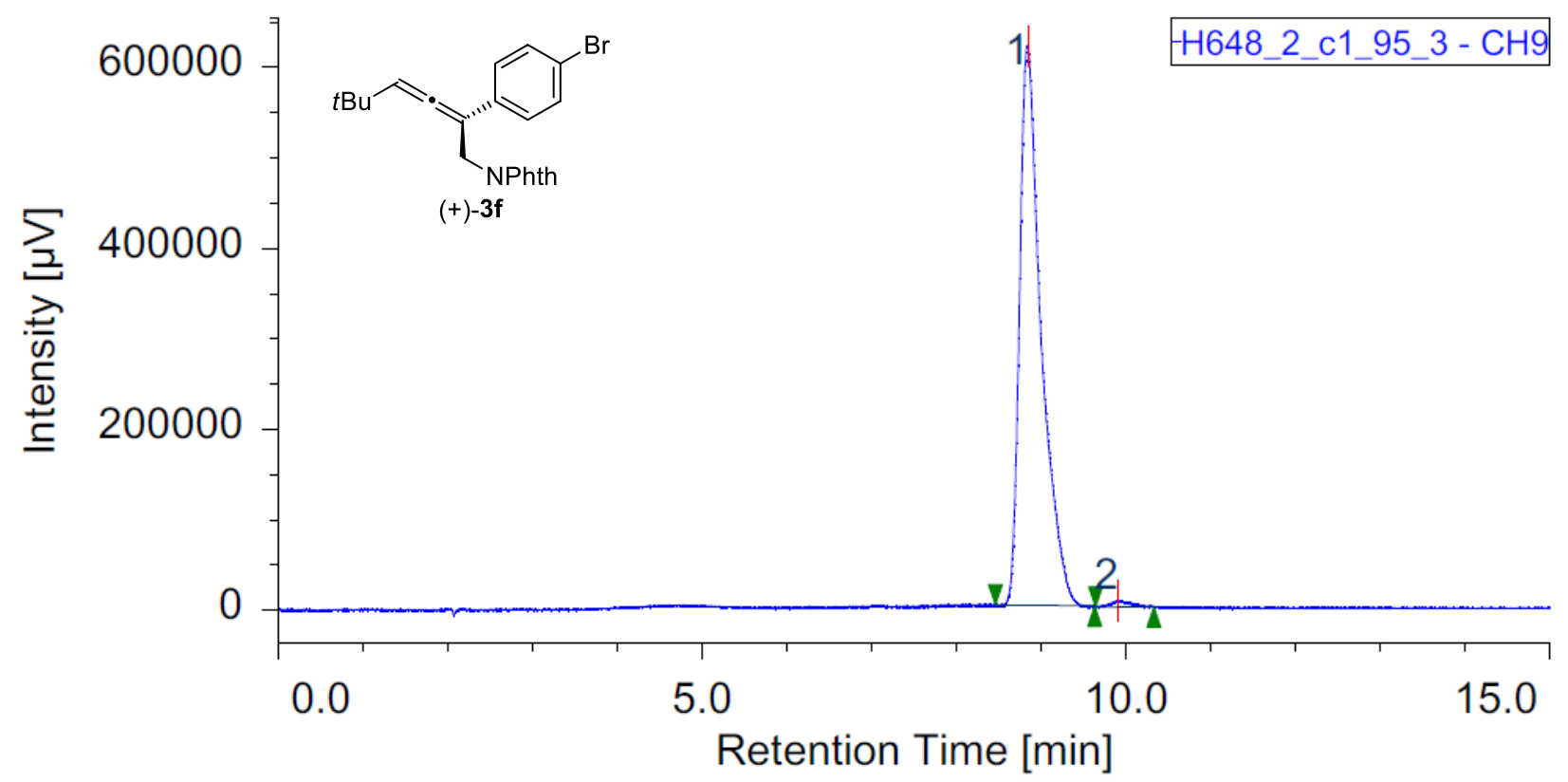

\begin{tabular}{|c|c|c|c|c|c|c|c|c|c|c|}
\hline Peak Name & $\mathrm{CH}$ & $t R[\min ]$ & Area $[\mu \mathrm{V} \cdot \mathrm{sec}]$ & Height $[\mu \mathrm{V}]$ & Area\% & Height $\%$ & Quantity & NTP & Resolution & Symmetry Factor \\
\hline Unknown & 9 & 8.840 & 10917057 & 617655 & 99.002 & 98.82 & $\mathrm{~N} / \mathrm{A}$ & 675 & 2.340 & 1.644 \\
\hline Unknown & 9 & 9.900 & 110025 & 7338 & 0.99 & 1.17 & $\mathrm{~N} / \mathrm{A}$ & 685 & $\mathrm{~N} / \mathrm{A}$ & 1.247 \\
\hline
\end{tabular}




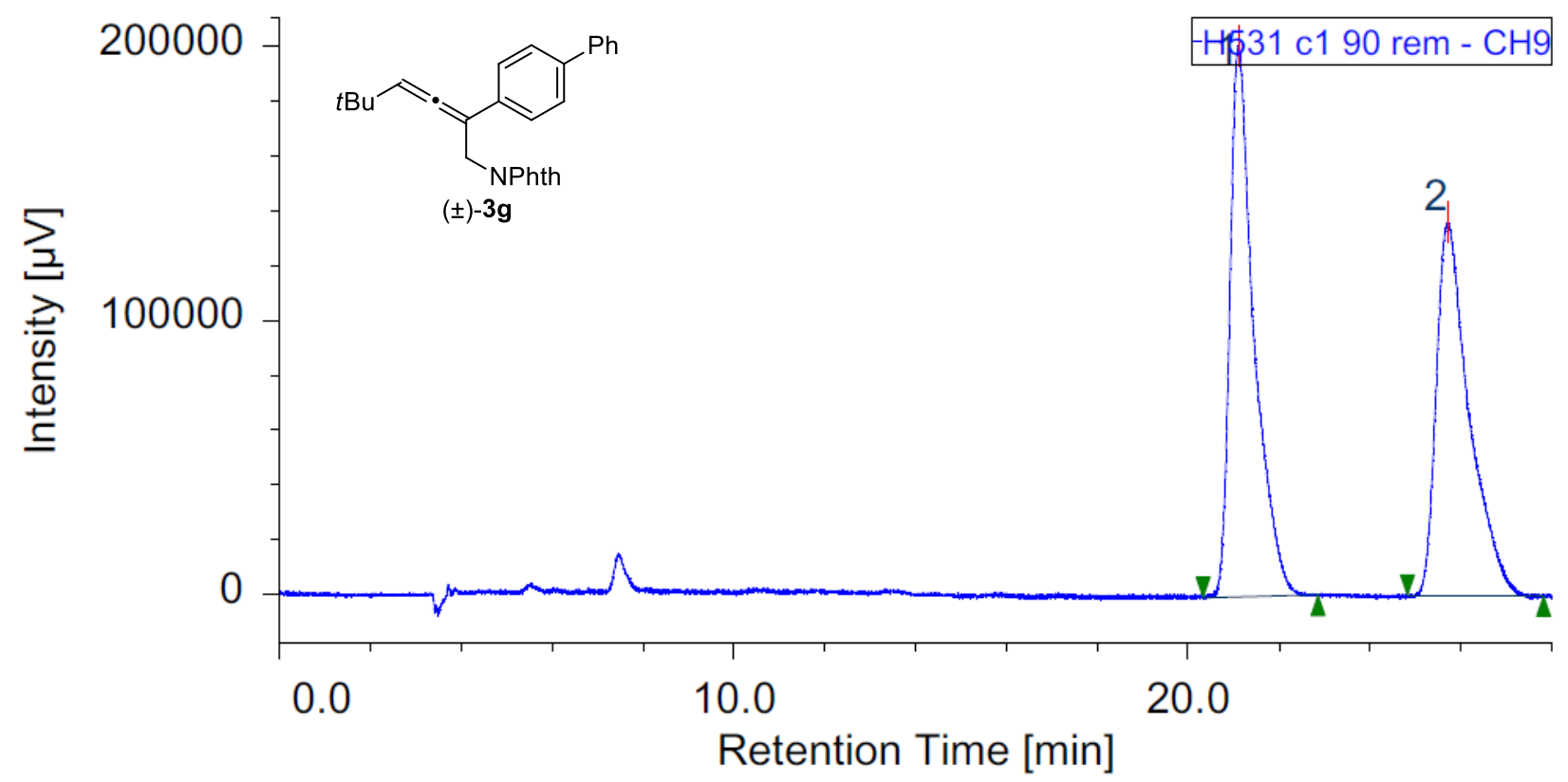

\begin{tabular}{|r|c|c|r|r|r|r|r|r|r|r|r|}
\hline$\#$ & Peak Name & $\mathrm{CH}$ & \multicolumn{1}{c|}{$\mathrm{R}[\mathrm{min}]$} & Area $[\mu \mathrm{V}$-sec] & Height $[\mu \mathrm{V}]$ & \multicolumn{1}{|c|}{ Area\% } & Height $\%$ & Quantity & NTP & Resolution & Symmetry Factor \\
\hline 1 & Unknown & 9 & 21.100 & 7404778 & 201253 & 51.986 & 59.613 & N/A & 9186 & 4.341 & 1.715 \\
\hline 2 & Unknown & 9 & 25.700 & 683893 & 136346 & 48.014 & 40.387 & N/A & 6825 & N/A & 1.717 \\
\hline
\end{tabular}

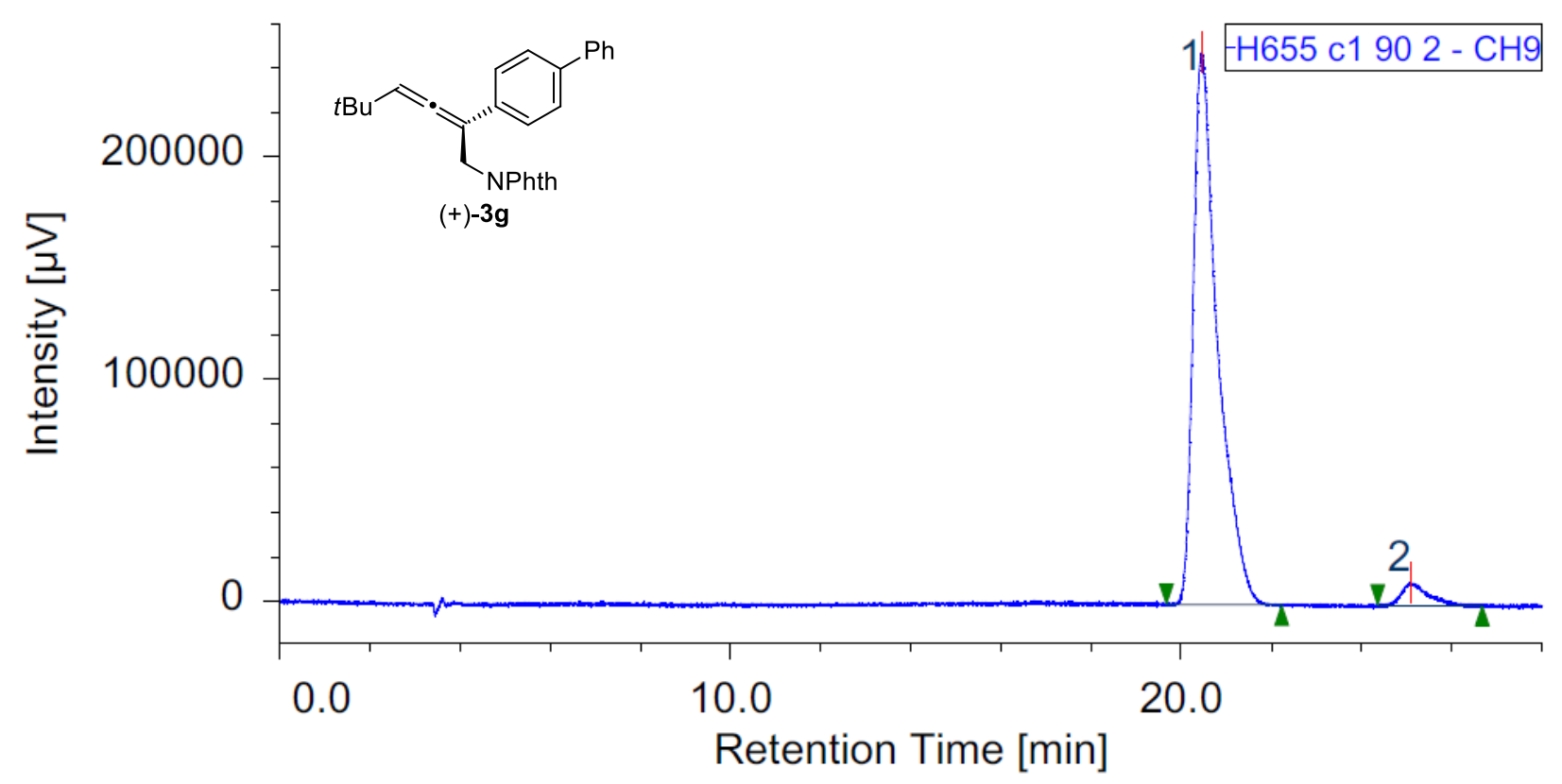

\begin{tabular}{|r|c|c|r|r|r|r|r|r|r|r|r|}
\hline$\#$ & Peak Name & $\mathrm{CH}$ & $\mathrm{tR}[\mathrm{min}]$ & Area $[\mu \mathrm{V}$-sec] & Height $[\mu \mathrm{V}]$ & Area $\%$ & Height $\%$ & Quantity & NTP & Resolution & Symmetry Factor \\
\hline 1 & Unknown & 9 & 20.453 & 9340140 & 248778 & 95.845 & 96.062 & N/A & 8233 & 4.842 & 1.744 \\
\hline 2 & Unknown & 9 & 25.080 & 404917 & 10199 & 4.155 & 3.938 & N/A & 9777 & N/A & 1.528 \\
\hline
\end{tabular}




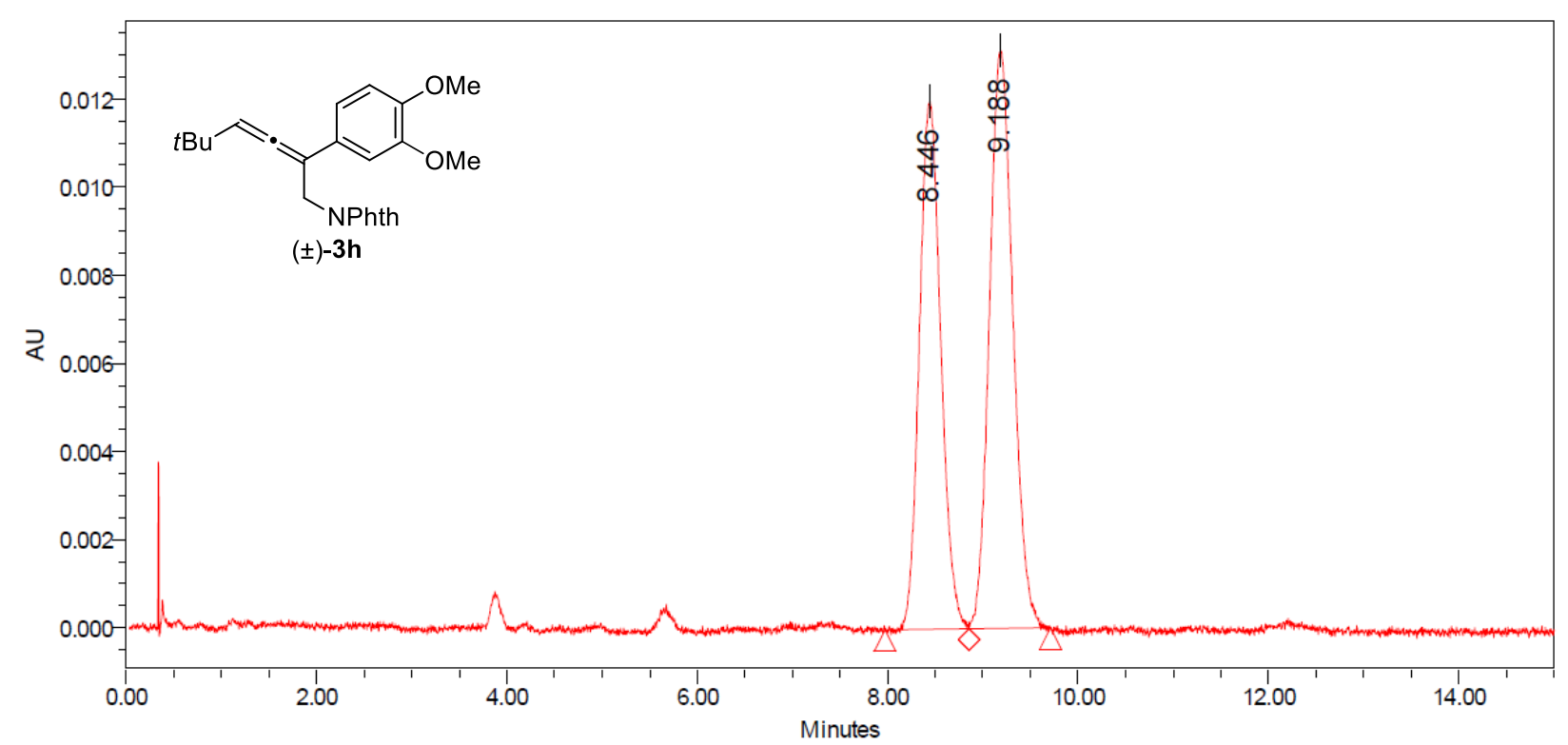

\begin{tabular}{|c|c|r|c|r|}
\hline & Processed Channel & $\begin{array}{r}\text { Retention } \\
\text { Time (min) }\end{array}$ & Area & $\%$ Area \\
\hline 1 & PDA Ch1 254nm@1.2nm -Compens. & 8.446 & 188396 & 45.59 \\
\hline 2 & PDA Ch1 254nm@1.2nm -Compens. & 9.188 & 224828 & 54.41 \\
\hline
\end{tabular}

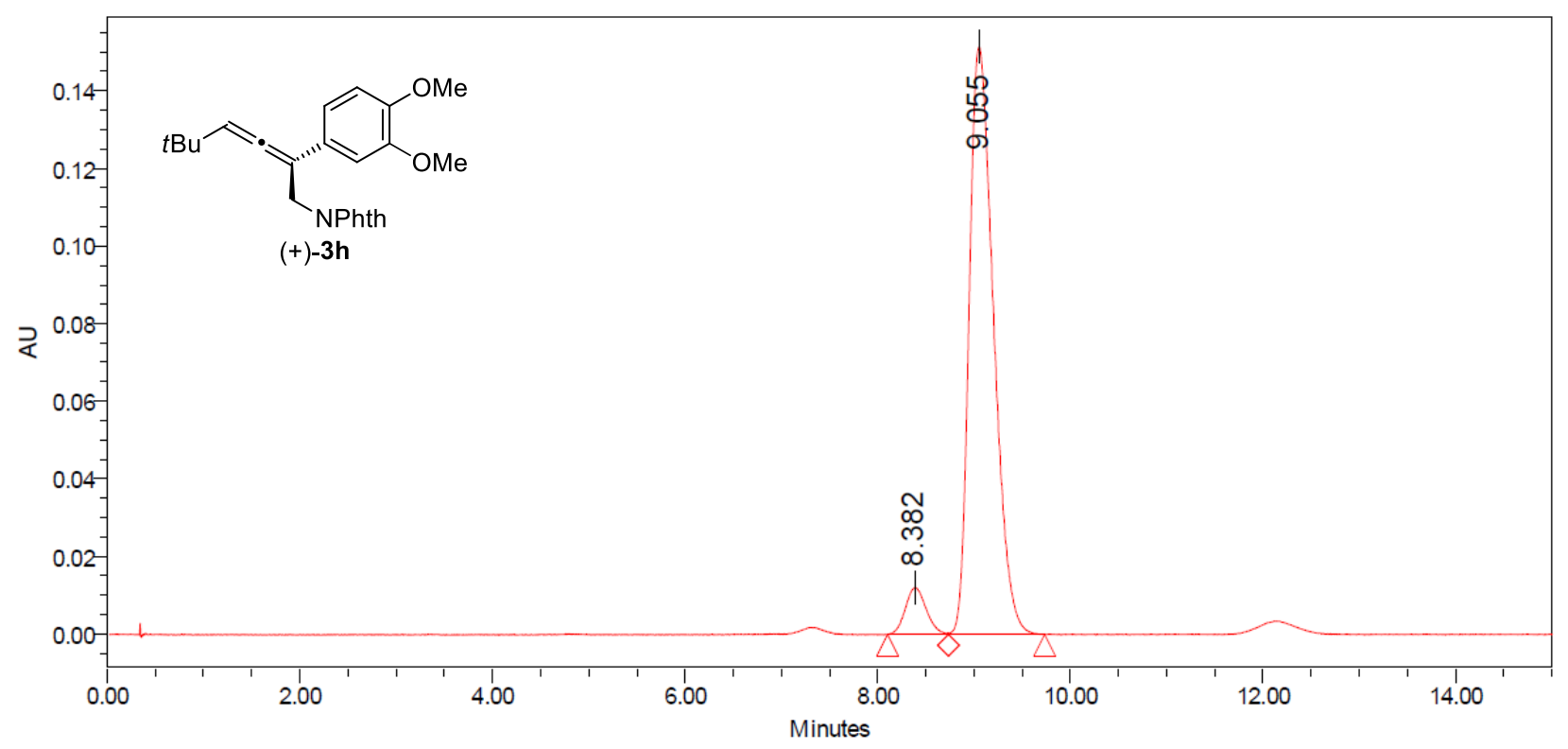

\begin{tabular}{|c|c|r|r|r|}
\hline & Processed Channel & $\begin{array}{r}\text { Retention } \\
\text { Time (min) }\end{array}$ & Area & $\%$ Area \\
\hline 1 & PDA Ch1 254nm@1.2nm -Compens. & 8.382 & 181708 & 6.41 \\
\hline 2 & PDA Ch1 254nm@1.2nm-Compens. & 9.055 & 2651802 & 93.59 \\
\hline
\end{tabular}




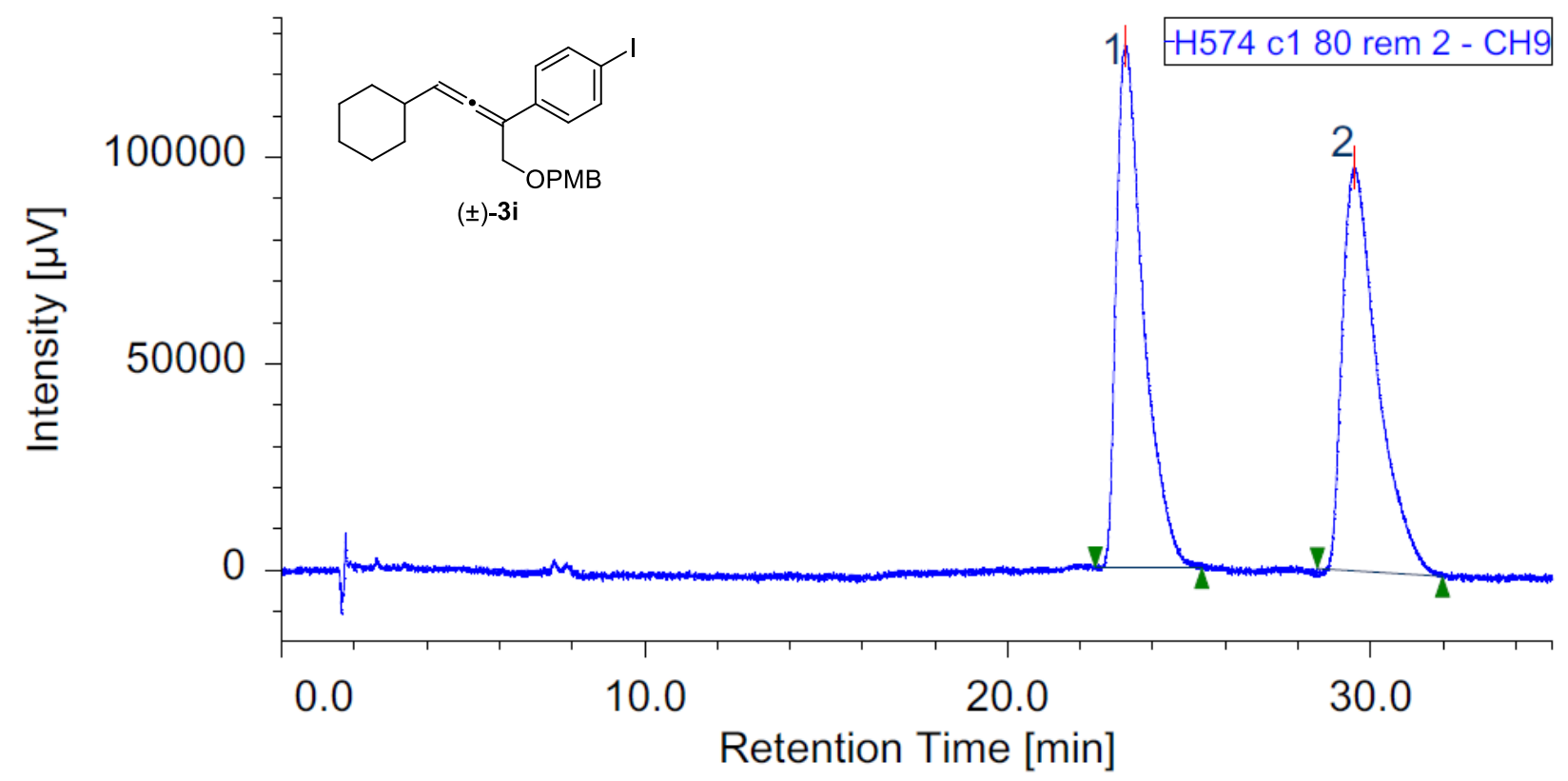

\begin{tabular}{|r|c|c|r|r|r|r|r|r|r|r|r|}
\hline$\#$ & Peak Name & $\mathrm{CH}$ & $\mathrm{tR}[\mathrm{min}]$ & Area $[\mu \mathrm{V} \cdot \mathrm{sec}]$ & Height $[\mu \mathrm{V}]$ & Area $\%$ & Height $\%$ & Quantity & NTP & Resolution & Symmetry Factor \\
\hline 1 & Unknown & 9 & 23.213 & 6587449 & 126352 & 50.135 & 56.410 & N/A & 5100 & 4.244 & 1.986 \\
\hline 2 & Unknown & 9 & 29.540 & 6552020 & 97636 & 49.865 & 43.590 & N/A & 4891 & N/A & 1.942 \\
\hline
\end{tabular}

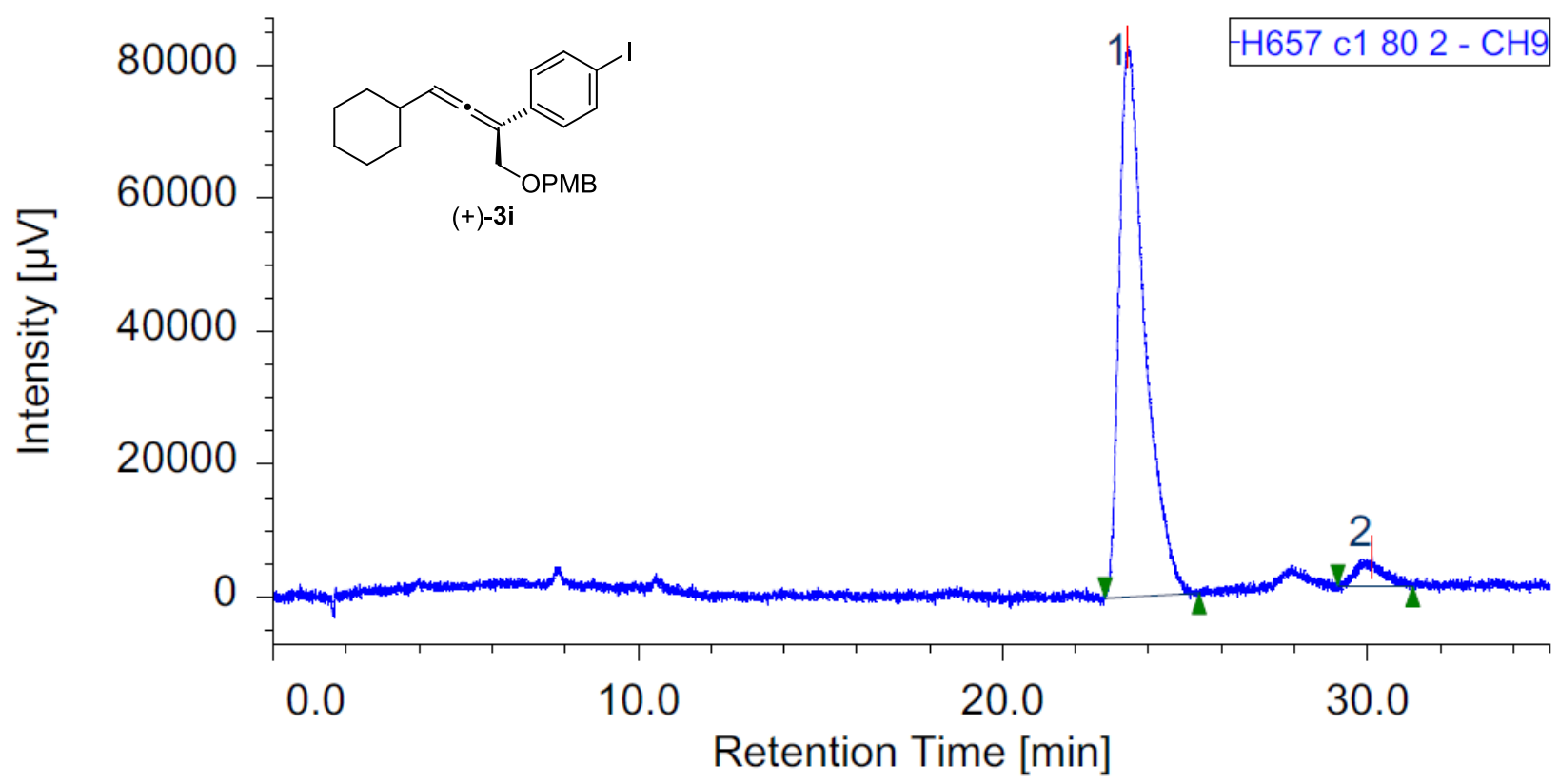

\begin{tabular}{|r|c|c|r|r|r|r|r|r|r|r|r|}
\hline$\#$ & Peak Name & $\mathrm{CH}$ & \multicolumn{1}{c|}{$\mathrm{R}[\mathrm{min}]$} & Area $[\mu \mathrm{V}$-sec] & Height $[\mu \mathrm{V}]$ & Area $\%$ & Height $\%$ & Quantity & NTP & Resolution & Symmetry Factor \\
\hline 1 & Unknown & 9 & 23.420 & 4089549 & 82833 & 95.415 & 94.977 & N/A & 5863 & 4.702 & 1.843 \\
\hline 2 & Unknown & 9 & 30.120 & 196496 & 4381 & 4.585 & 5.023 & N/A & 5438 & N/A & \\
\hline
\end{tabular}




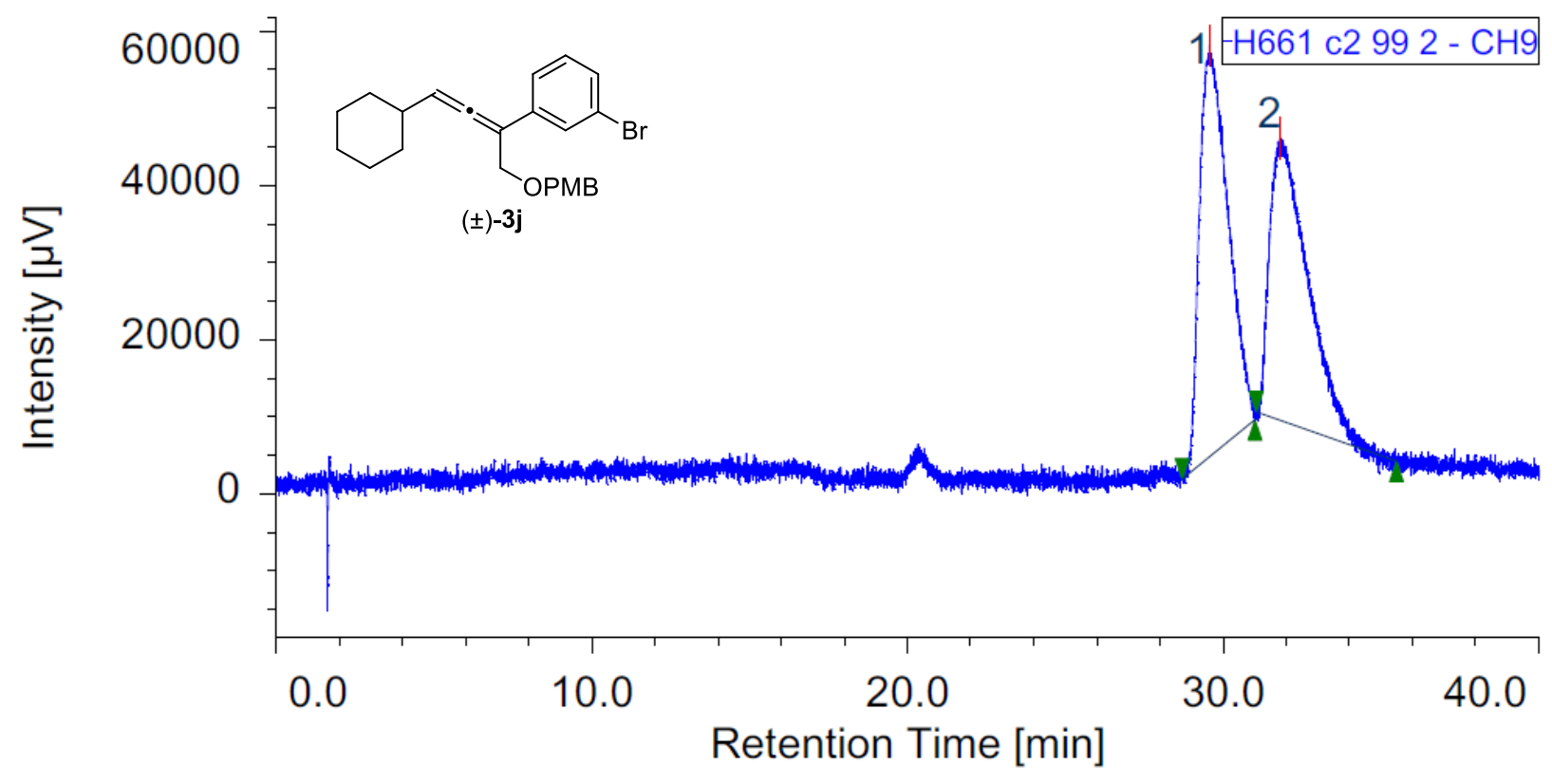

\begin{tabular}{|r|c|c|r|r|r|r|r|r|r|r|r|}
\hline$\#$ & Peak Name & $\mathrm{CH}$ & \multicolumn{1}{|c}{$[\mathrm{min}]$} & Area $[\mu \mathrm{V}$-sec] & Height $[\mu \mathrm{V}]$ & Area $\%$ & Height\% & Quantity & NTP & Resolution & Symmetry Factor \\
\hline 1 & Unknown & 9 & 29.553 & 3316863 & 53385 & 51.609 & 59.302 & N/A & 4671 & 1.116 & 1.625 \\
\hline 2 & Unknown & 9 & 31.813 & 3109987 & 36637 & 48.391 & 40.698 & N/A & 2977 & N/A & 2.342 \\
\hline
\end{tabular}

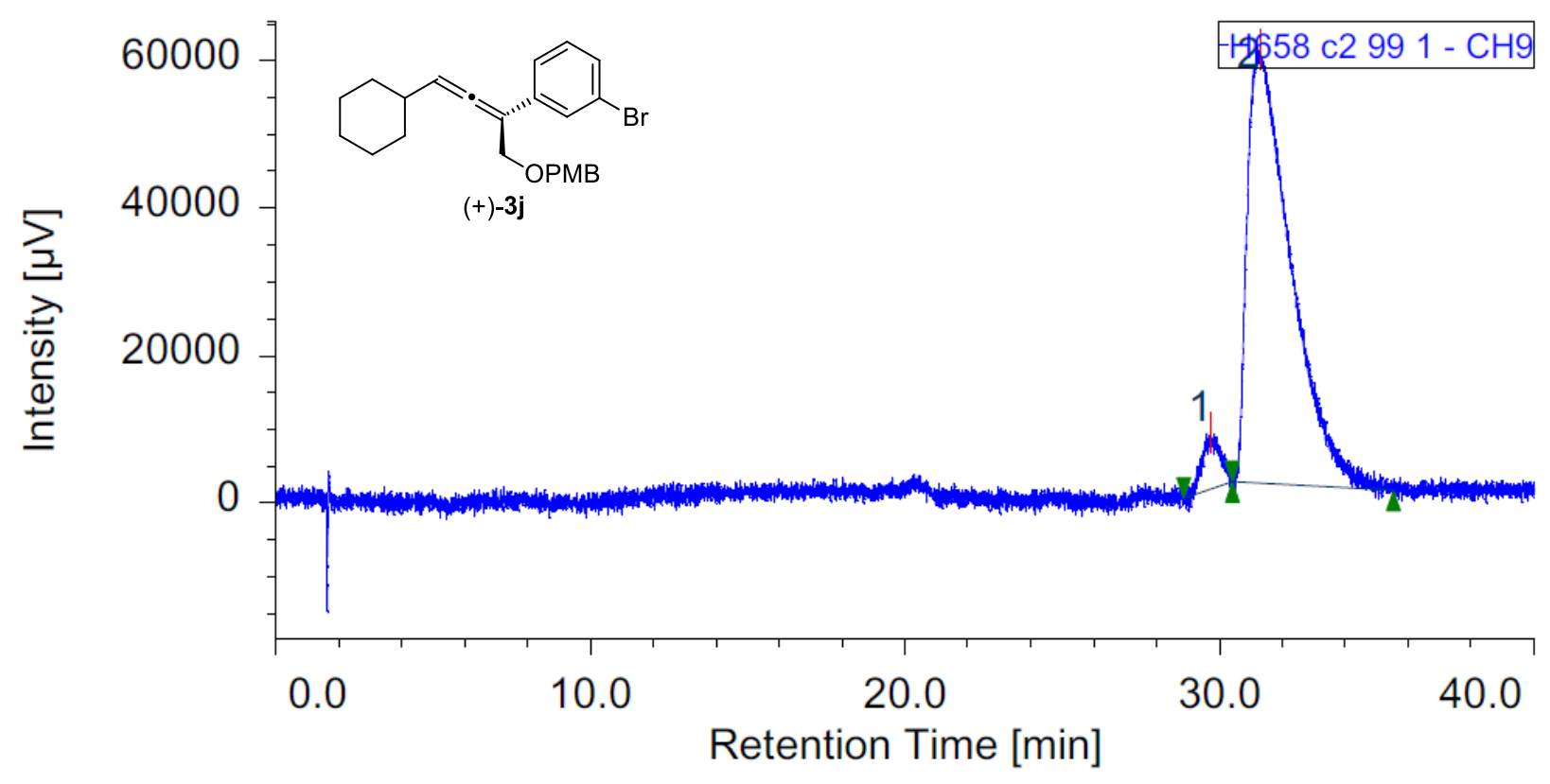

\begin{tabular}{|r|c|c|r|r|r|r|r|r|r|r|r|}
\hline$\#$ & Peak Name & $\mathrm{CH}$ & \multicolumn{1}{|c|}{$[\mathrm{min}]$} & Area $[\mu \mathrm{V}-\mathrm{sec}]$ & Height $[\mu \mathrm{V}]$ & Area\% & Height $\%$ & Quantity & NTP & Resolution & Symmetry Factor \\
\hline 1 & Unknown & 9 & 29.720 & 301006 & 7671 & 5.046 & 11.530 & N/A & 7956 & 0.805 & 0.896 \\
\hline 2 & Unknown & 9 & 31.273 & 5663954 & 58860 & 94.954 & 88.470 & N/A & 2432 & N/A & 2.617 \\
\hline
\end{tabular}




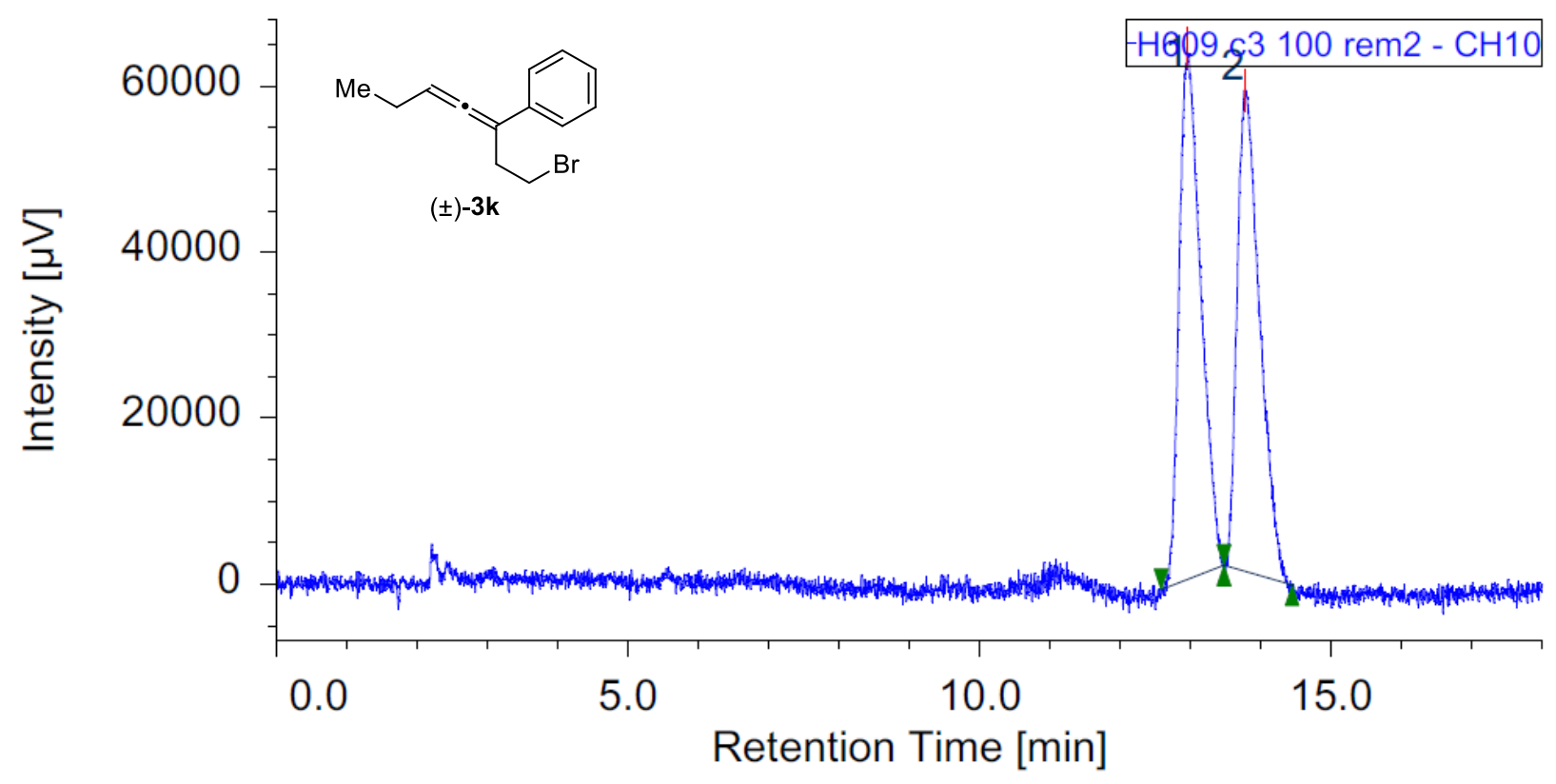

\begin{tabular}{|r|c|c|r|r|r|r|r|r|r|r|r|}
\hline$\#$ & Peak Name & $\mathrm{CH}$ & \multicolumn{1}{|c|}{$[\mathrm{min}]$} & Area $[\mu \mathrm{V}-\mathrm{sec}]$ & Height $[\mu \mathrm{V}]$ & \multicolumn{1}{|c|}{ Area\% } & Height $\%$ & Quantity & NTP & Resolution & Symmetry Factor \\
\hline 1 & Unknown & 10 & 12.947 & 1352694 & 64051 & 51.192 & 52.551 & N/A & 8485 & 1.423 & 1.481 \\
\hline 2 & Unknown & 10 & 13.767 & 1289716 & 57833 & 48.808 & 47.449 & N/A & 8611 & N/A & 1.622 \\
\hline
\end{tabular}

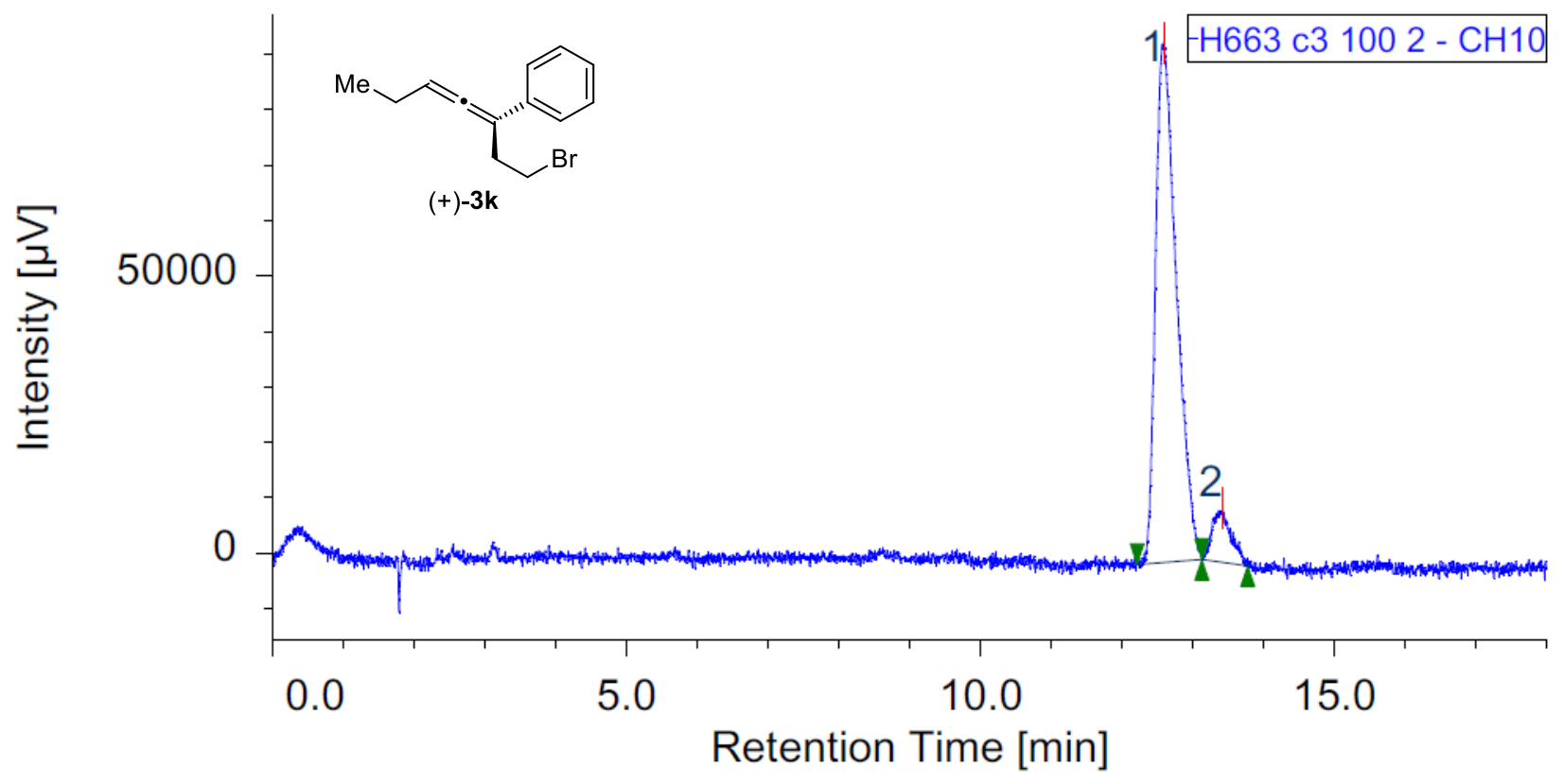

\begin{tabular}{|r|c|r|r|r|r|r|r|r|r|r|r|}
\hline$\#$ & Peak Name & $\mathrm{CH}$ & \multicolumn{1}{|c|}{$[\mathrm{min}]$} & Area $[\mu \mathrm{V}-\mathrm{sec}]$ & Height $[\mu \mathrm{V}]$ & Area $\%$ & Height $\%$ & Quantity & \multicolumn{1}{c|}{ NTP } & Resolution & Symmetry Factor \\
\hline 1 & Unknown & 10 & 12.587 & 1921346 & 93854 & 91.490 & 90.536 & N/A & 8653 & 1.552 & 1.493 \\
\hline 2 & Unknown & 10 & 13.420 & 178722 & 9811 & 8.510 & 9.464 & N/A & 10046 & N/A & 1.105 \\
\hline
\end{tabular}


VII. NMR Spectra

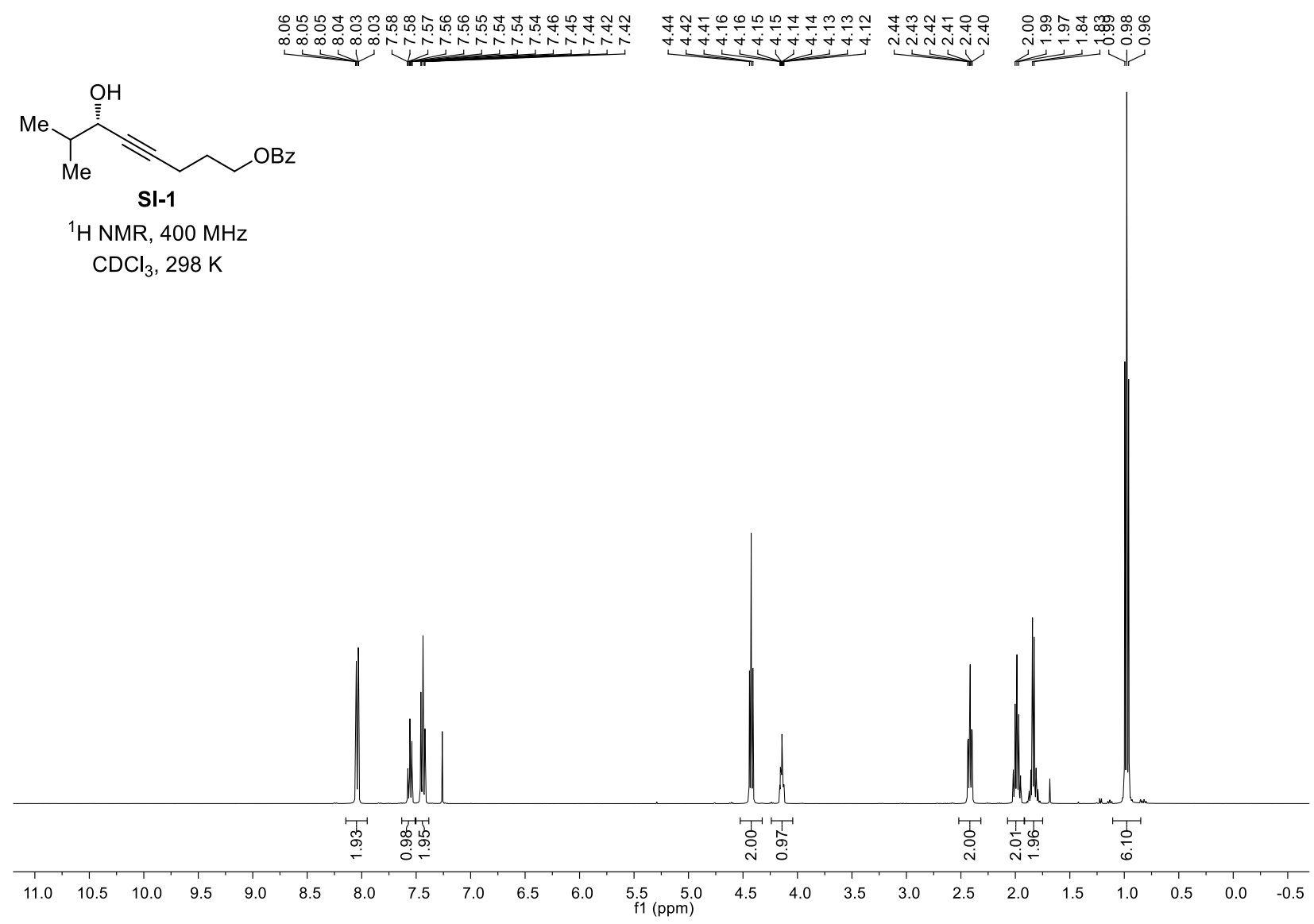

li<smiles>CC(C)C(O)C#CCCCOC(=O)c1ccccc1</smiles>

${ }^{13} \mathrm{C}$ NMR, $101 \mathrm{MHz}$

$\mathrm{CDCl}_{3}, 298 \mathrm{~K}$

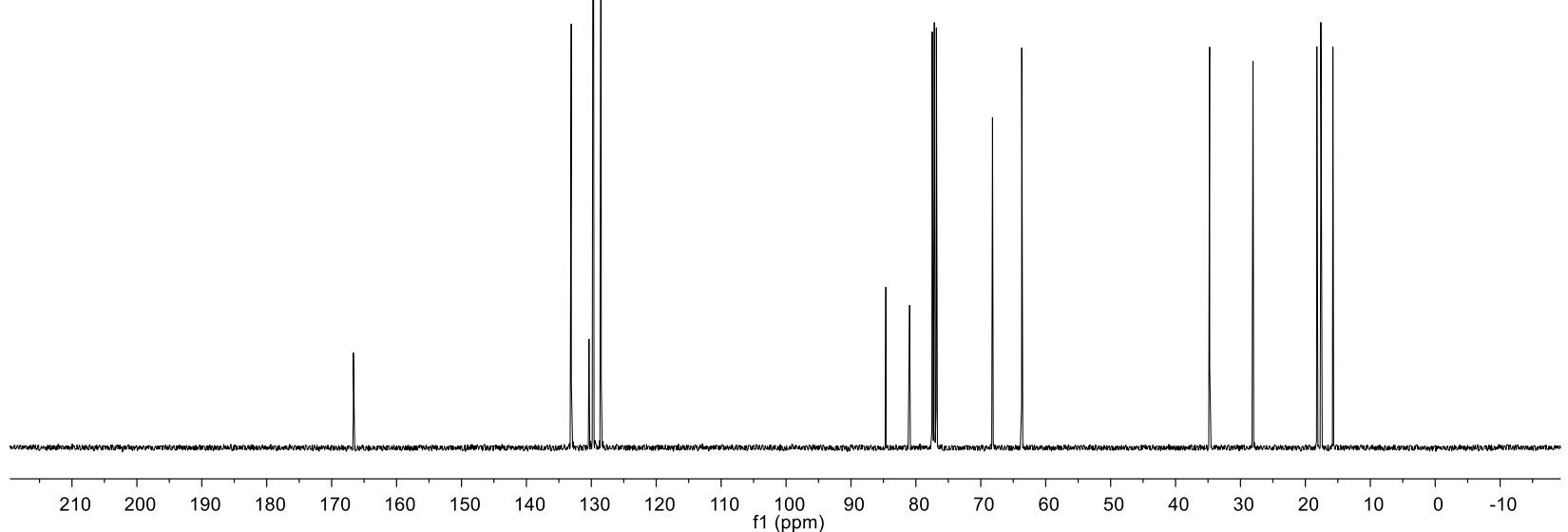




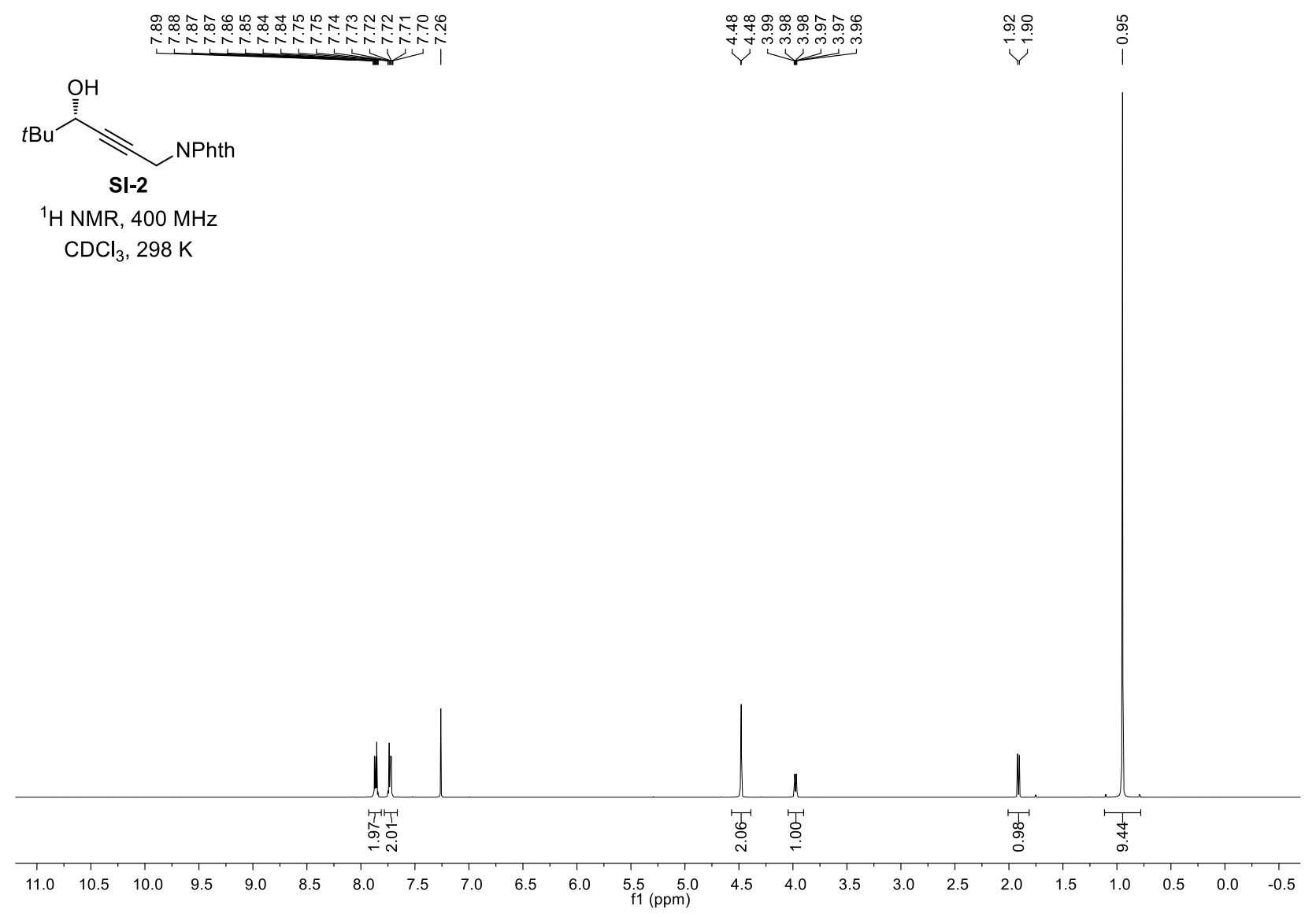

NNPhth

SI-2

${ }^{13} \mathrm{C}$ NMR, $101 \mathrm{MHz}$

$\mathrm{CDCl}_{3}, 298 \mathrm{~K}$

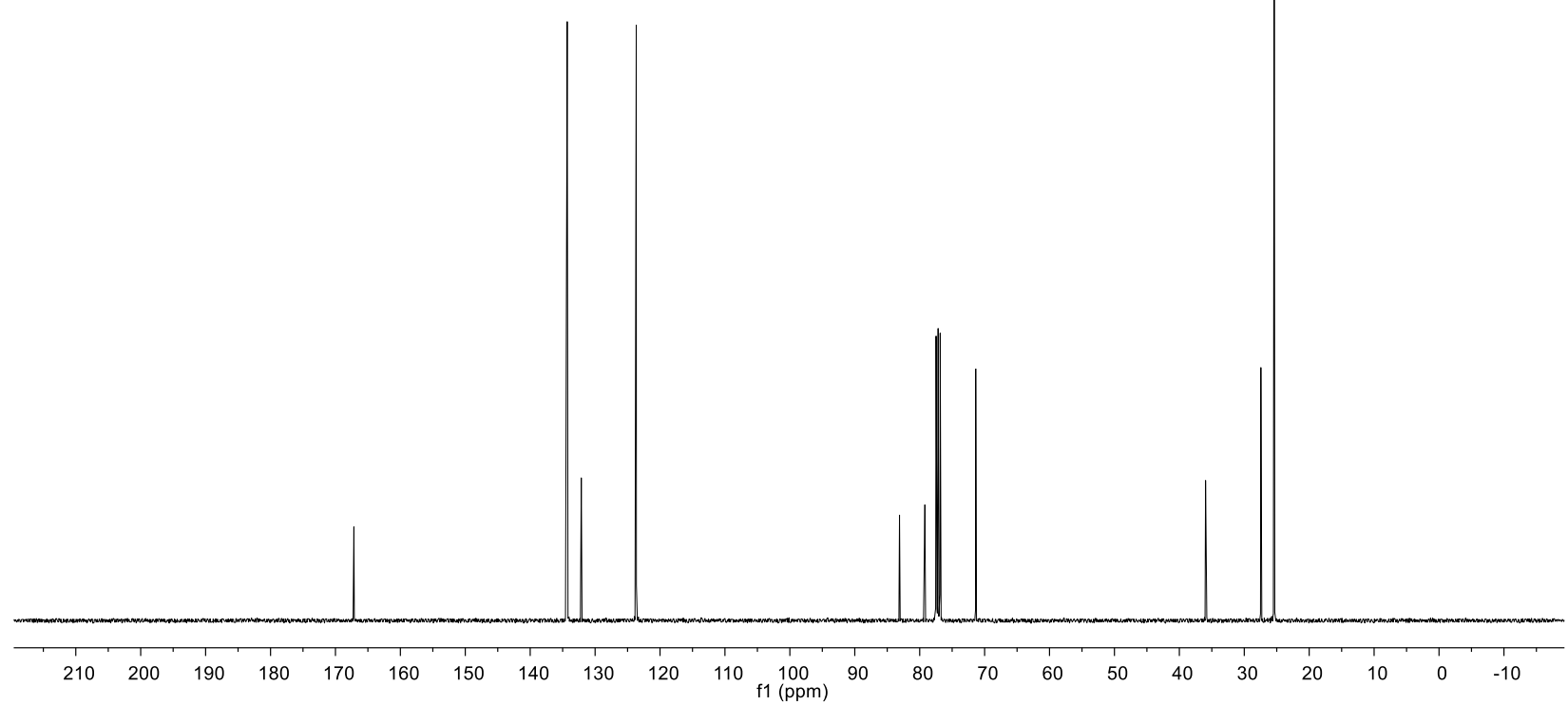




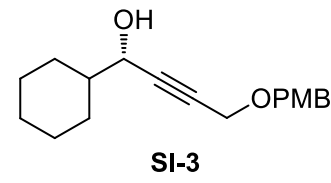

${ }^{1} \mathrm{H}$ NMR, $400 \mathrm{MHz}$ $\mathrm{CDCl}_{3}, 298 \mathrm{~K}$

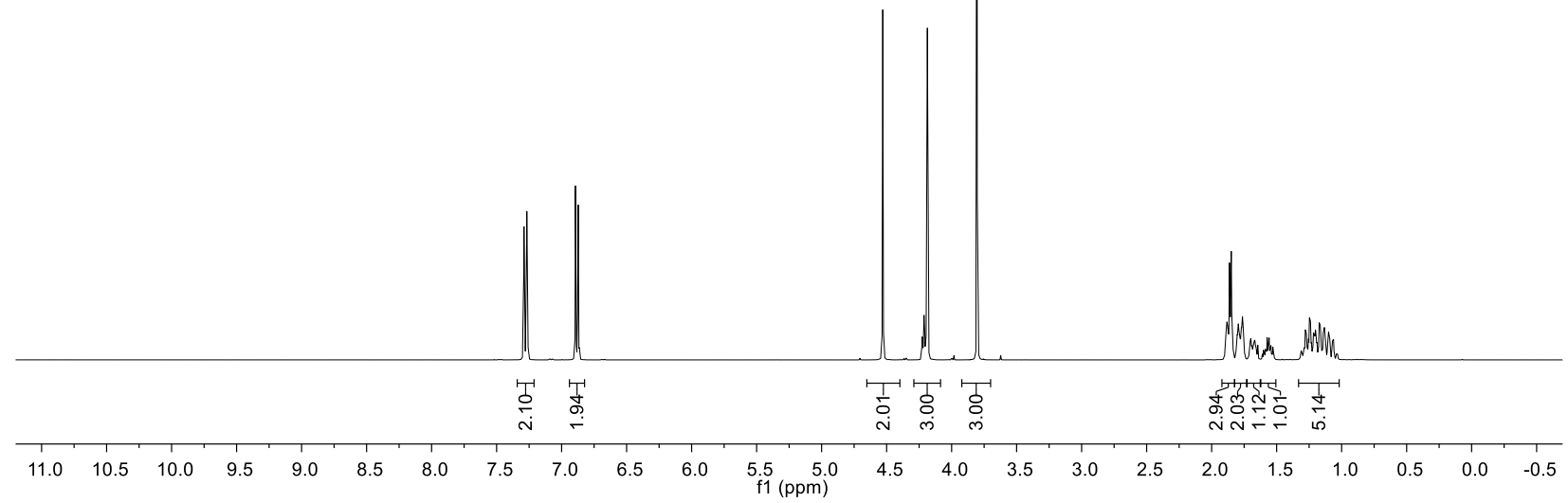<smiles>CCOCOCC#C[C@@H](O)C1CCCCC1</smiles>

SI-3

${ }^{13} \mathrm{C}$ NMR, $101 \mathrm{MHz}$ $\mathrm{CDCl}_{3}, 298 \mathrm{~K}$
光

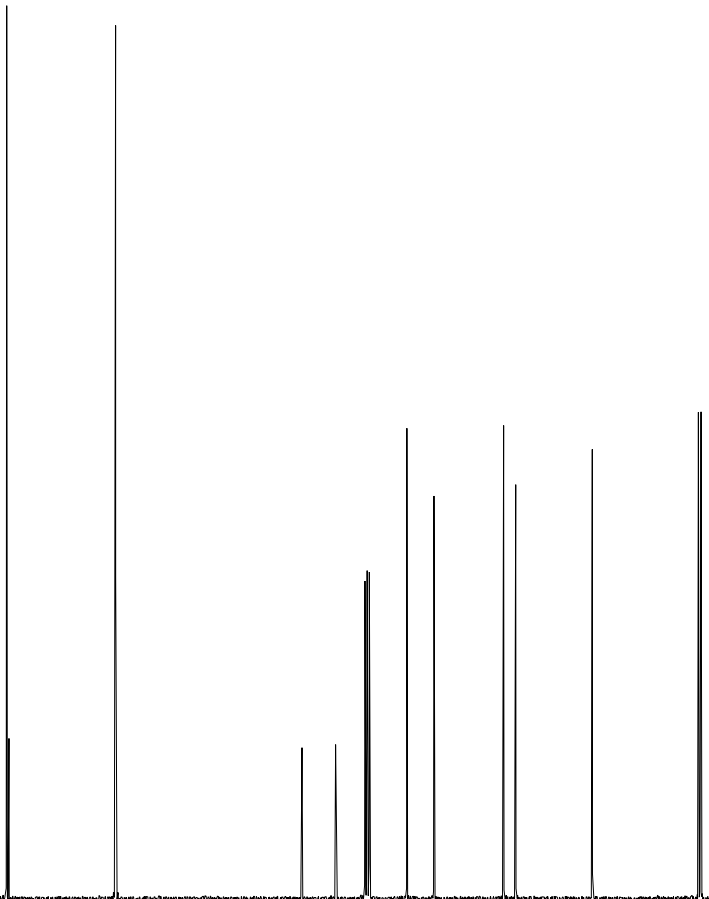

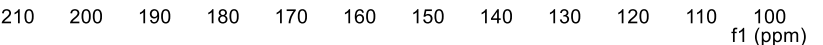




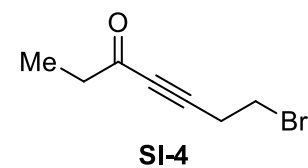

SI-4

${ }^{1} \mathrm{H}$ NMR, $400 \mathrm{MHz}$

$\mathrm{CDCl}_{3}, 298 \mathrm{~K}$

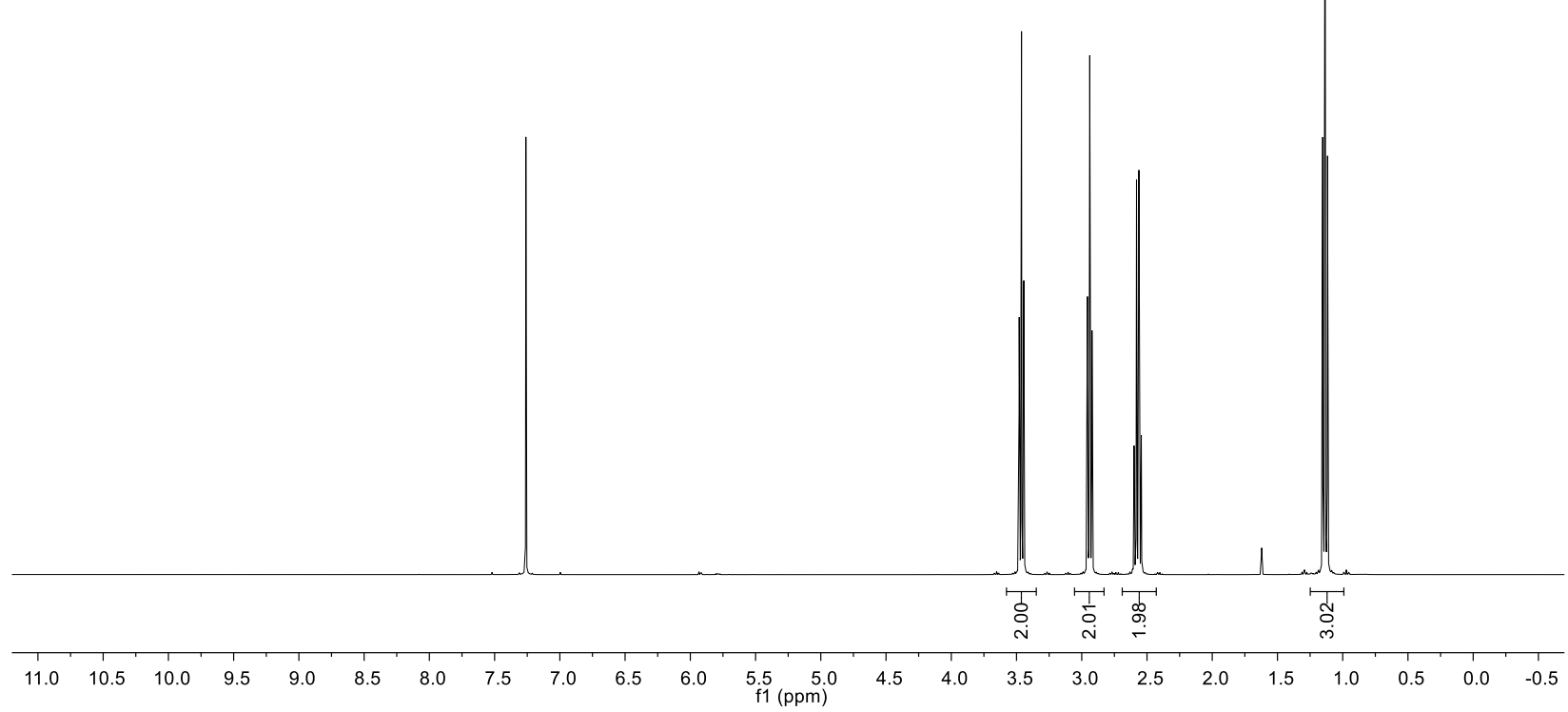

$\stackrel{\substack{\infty \\ \infty}}{\infty}$

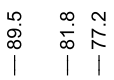

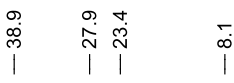

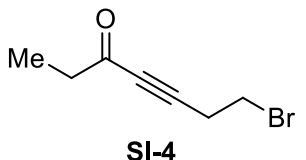

${ }^{13} \mathrm{C}$ NMR, $101 \mathrm{MHz}$

$\mathrm{CDCl}_{3}, 298 \mathrm{~K}$

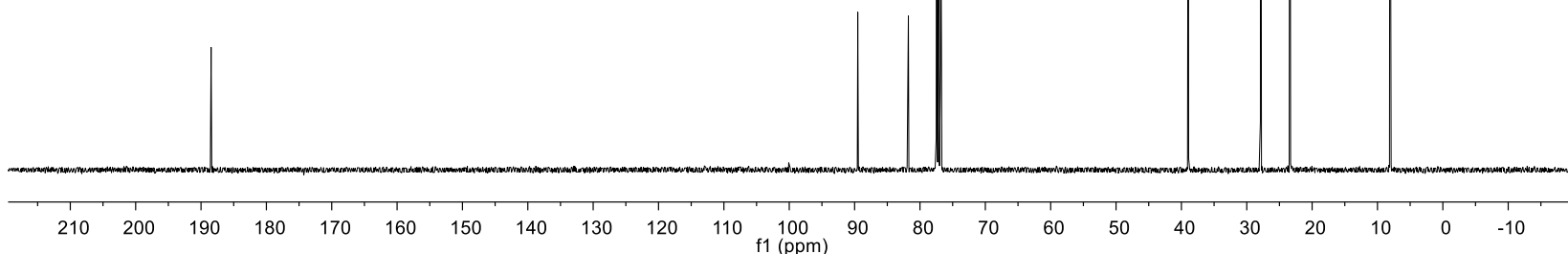




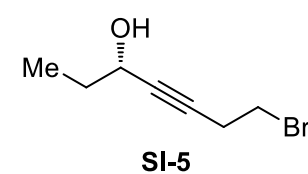

$\mathrm{SI}-5$

${ }^{1} \mathrm{H}$ NMR, $400 \mathrm{MHz}$

$\mathrm{CDCl}_{3}, 298 \mathrm{~K}$
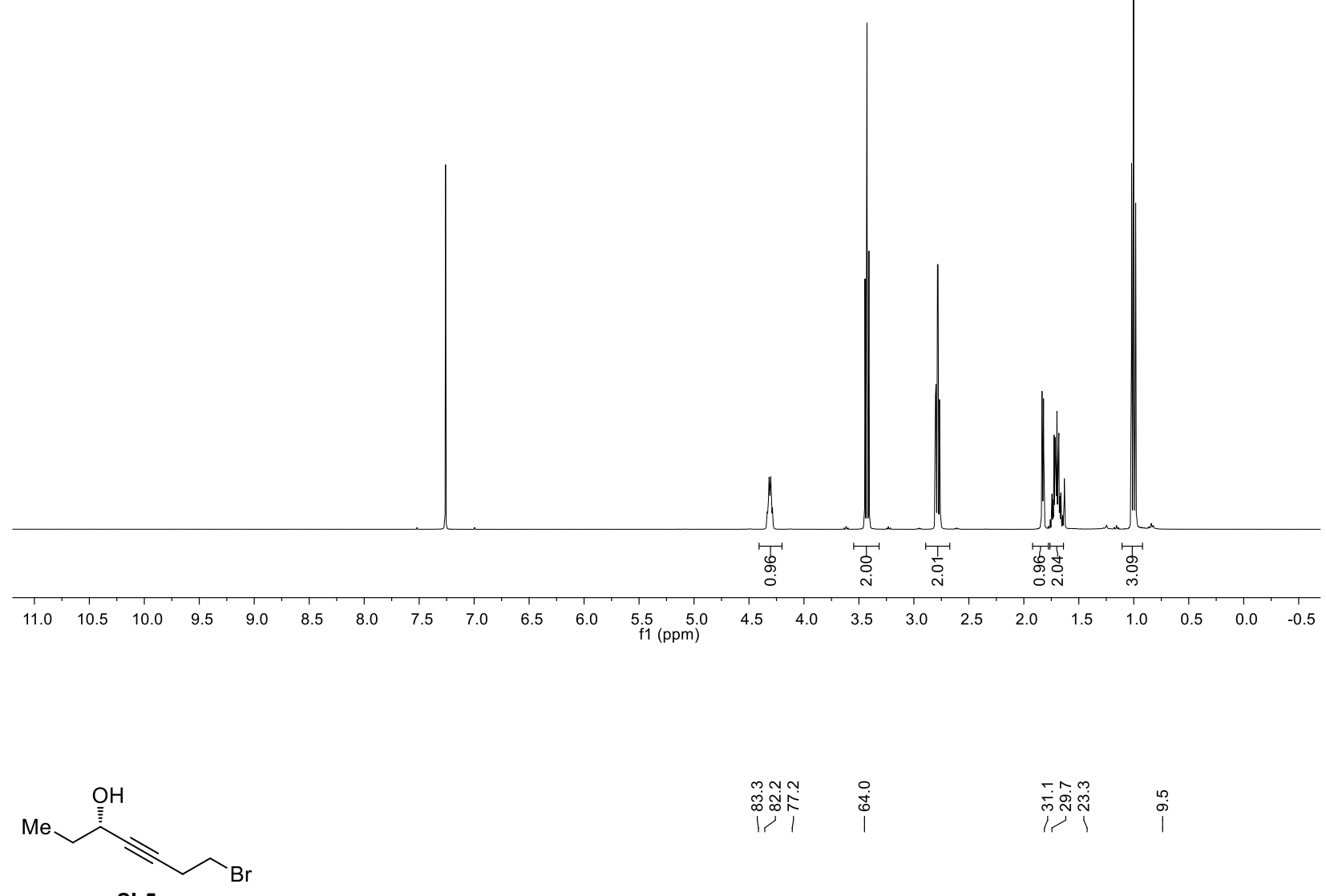

${ }^{13} \mathrm{C}$ NMR, $101 \mathrm{MHz}$

$\mathrm{CDCl}_{3}, 298 \mathrm{~K}$

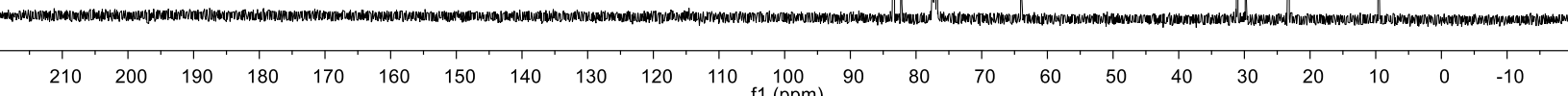




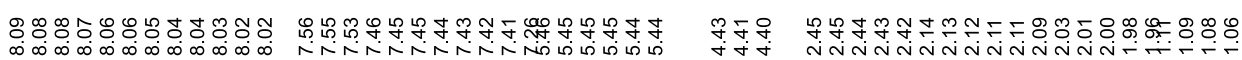

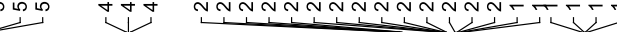

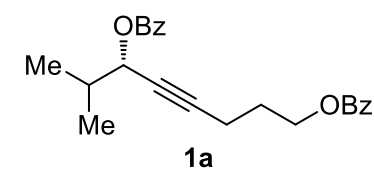

${ }^{1} \mathrm{H}$ NMR, $400 \mathrm{MHz}$

$\mathrm{CDCl}_{3}, 298 \mathrm{~K}$

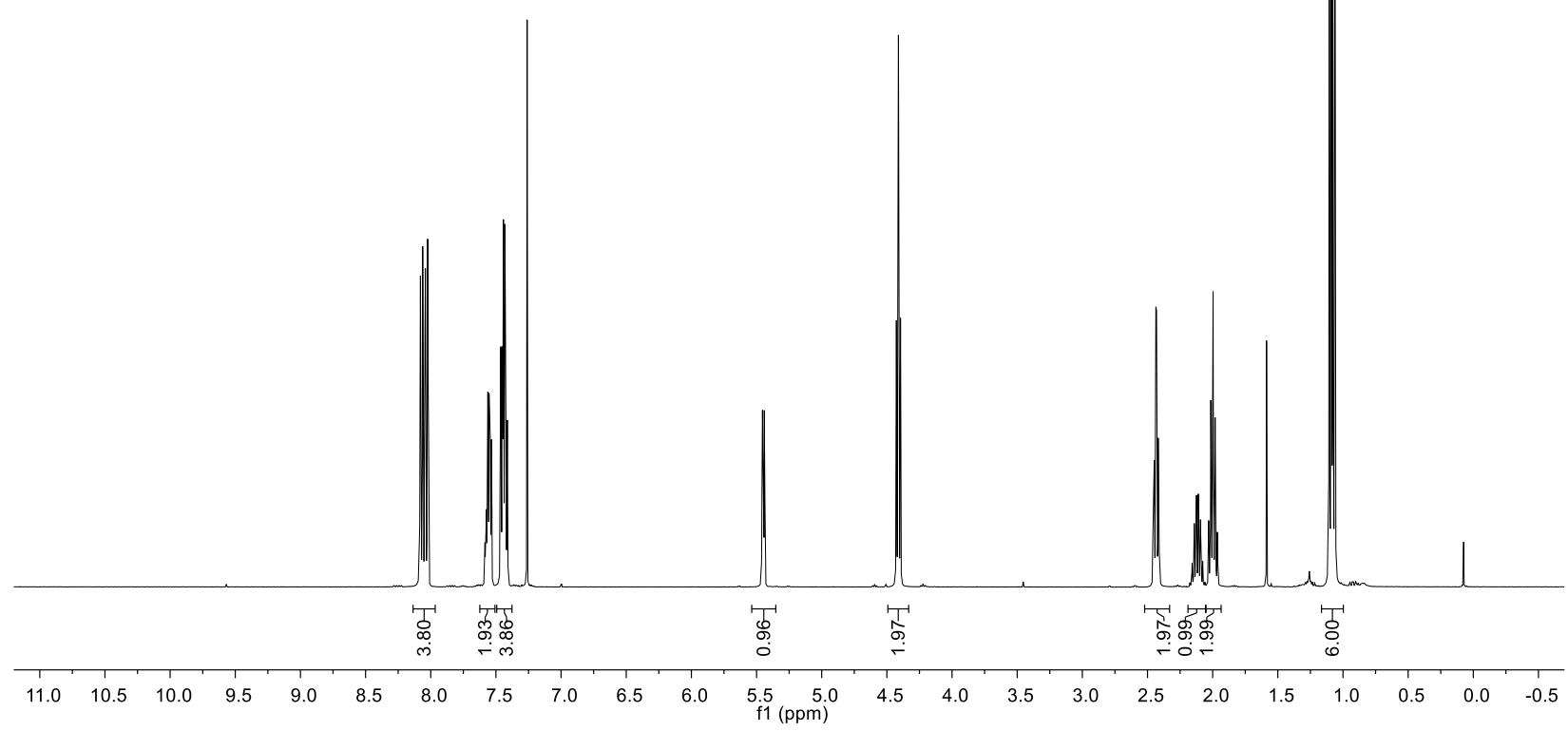

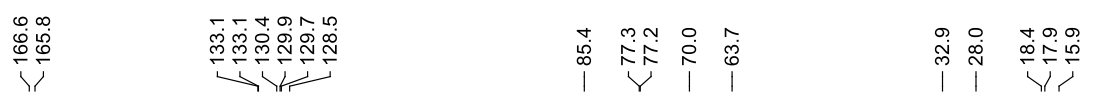<smiles>CC(C)[C@H](C#CCCCOC(C)(C)C)OC(C)(C)C</smiles>

${ }^{13} \mathrm{C}$ NMR, $101 \mathrm{MHz}$

$\mathrm{CDCl}_{3}, 298 \mathrm{~K}$ 


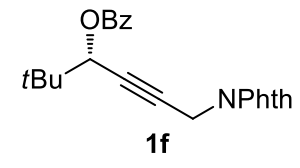

${ }^{1} \mathrm{H} \mathrm{NMR}, 400 \mathrm{MHz}$

$\mathrm{CDCl}_{3}, 298 \mathrm{~K}$
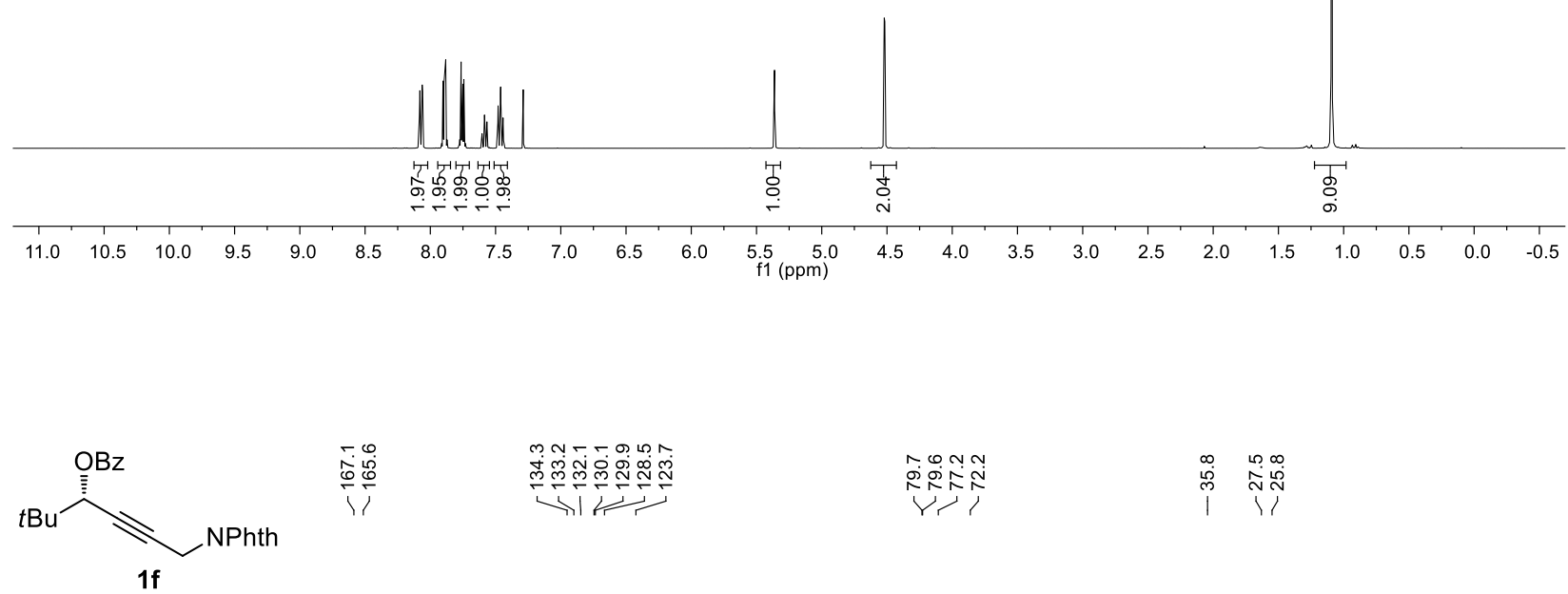

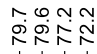

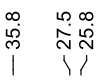

${ }^{13} \mathrm{C}$ NMR, $101 \mathrm{MHz}$

$\mathrm{CDCl}_{3}, 298 \mathrm{~K}$

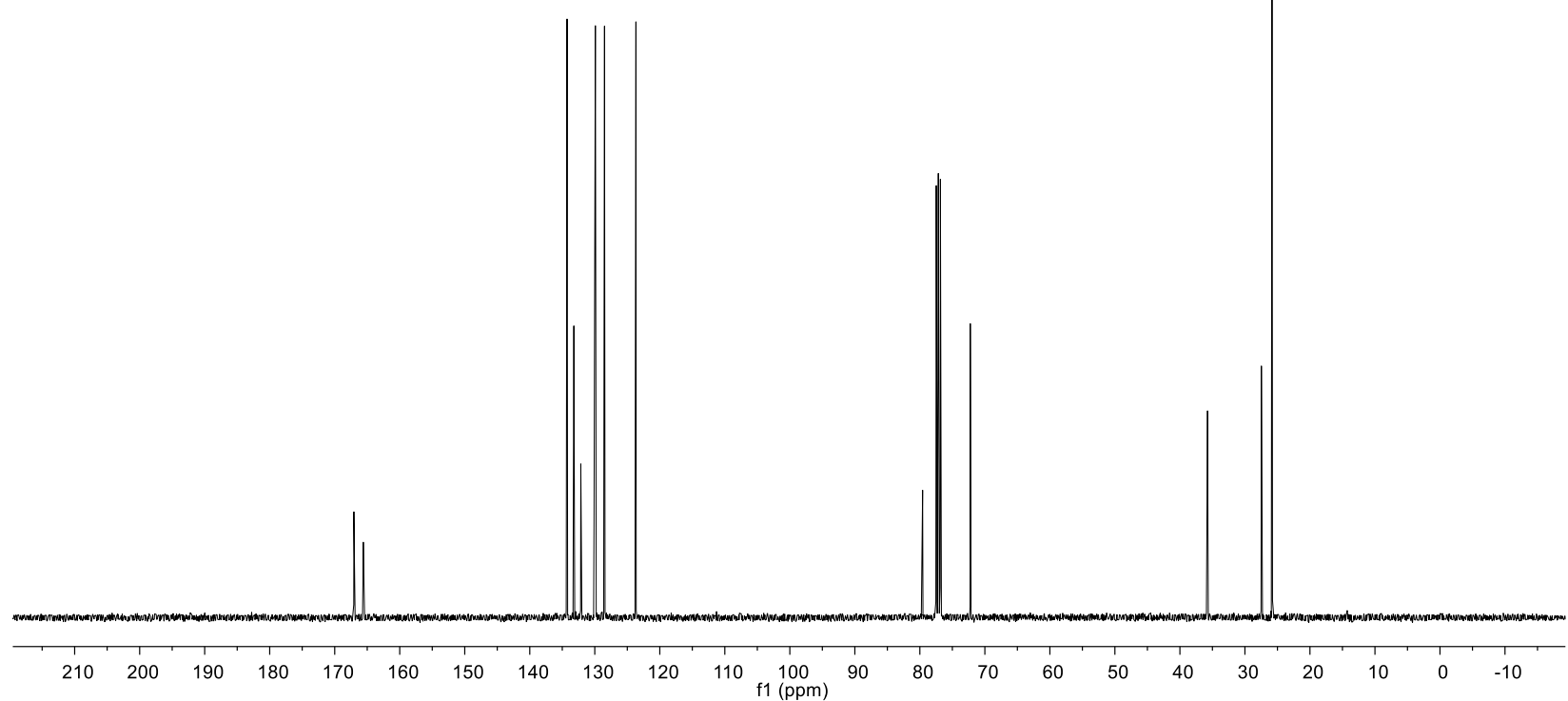




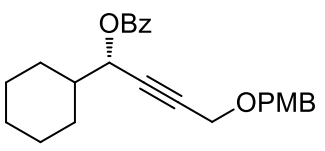

$1 \mathrm{i}$

${ }^{1} \mathrm{H}$ NMR, $400 \mathrm{MHz}$

$\mathrm{CDCl}_{3}, 298 \mathrm{~K}$
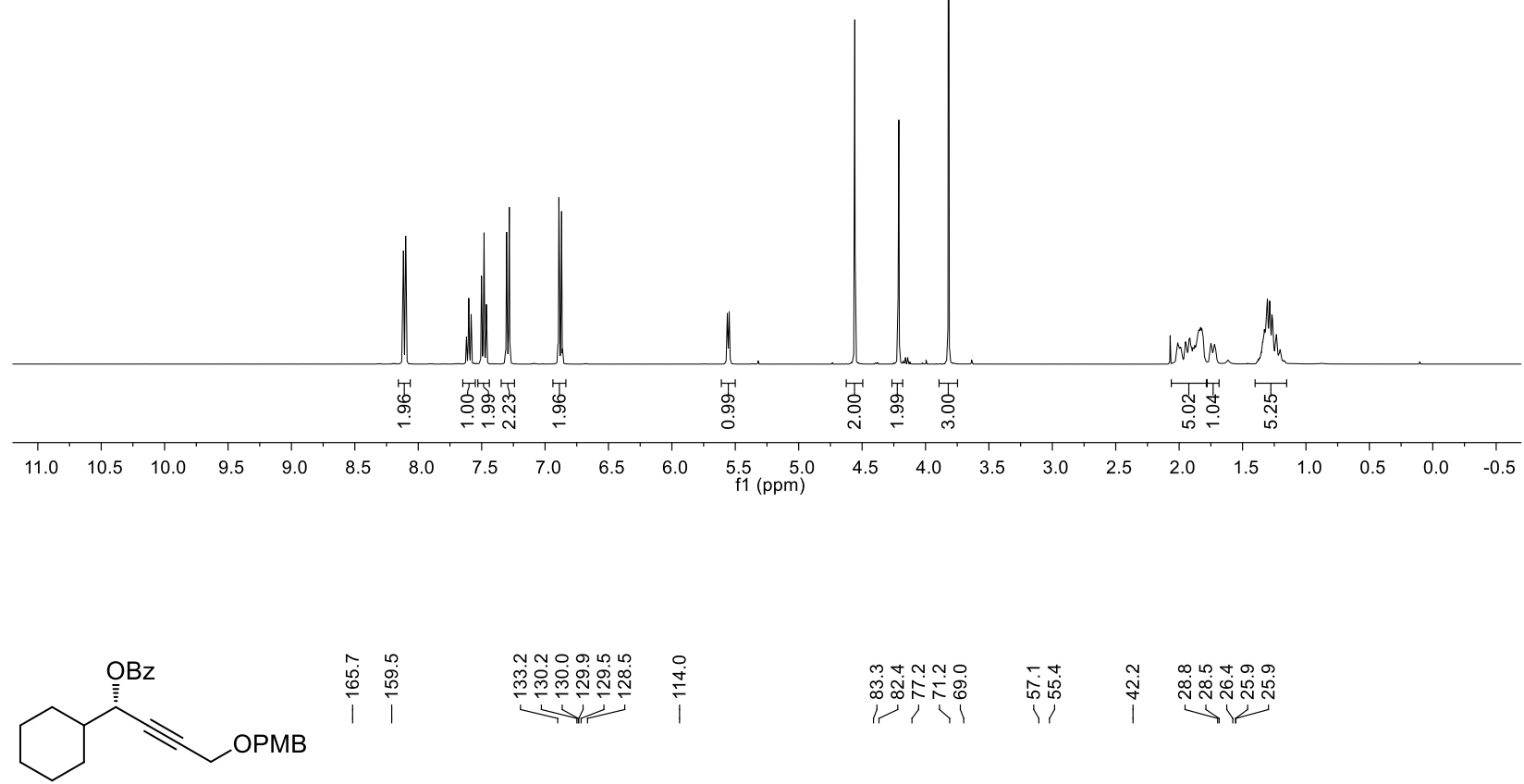

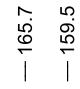
|

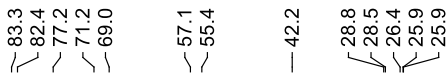

$1 \mathrm{i}$

${ }^{13} \mathrm{C}$ NMR, $101 \mathrm{MHz}$ $\mathrm{CDCl}_{3}, 298 \mathrm{~K}$

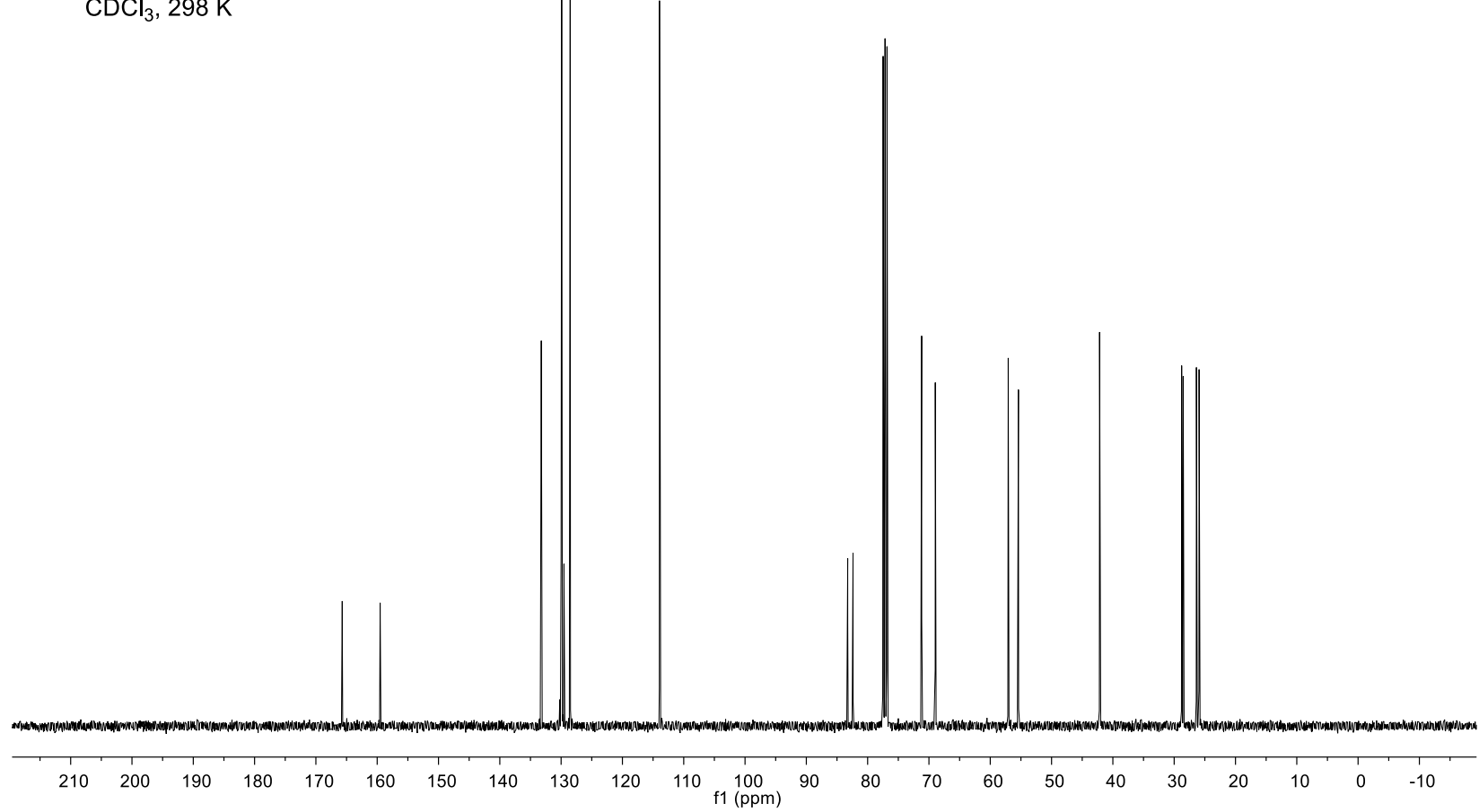




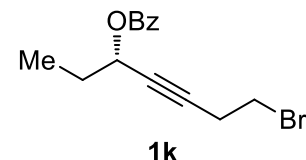

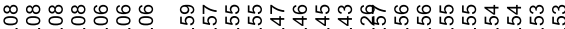

$1 \mathrm{k}$

${ }^{1} \mathrm{H}$ NMR, $400 \mathrm{MHz}$

$\mathrm{CDCl}_{3}, 298 \mathrm{~K}$

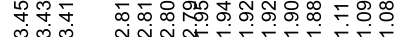

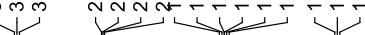
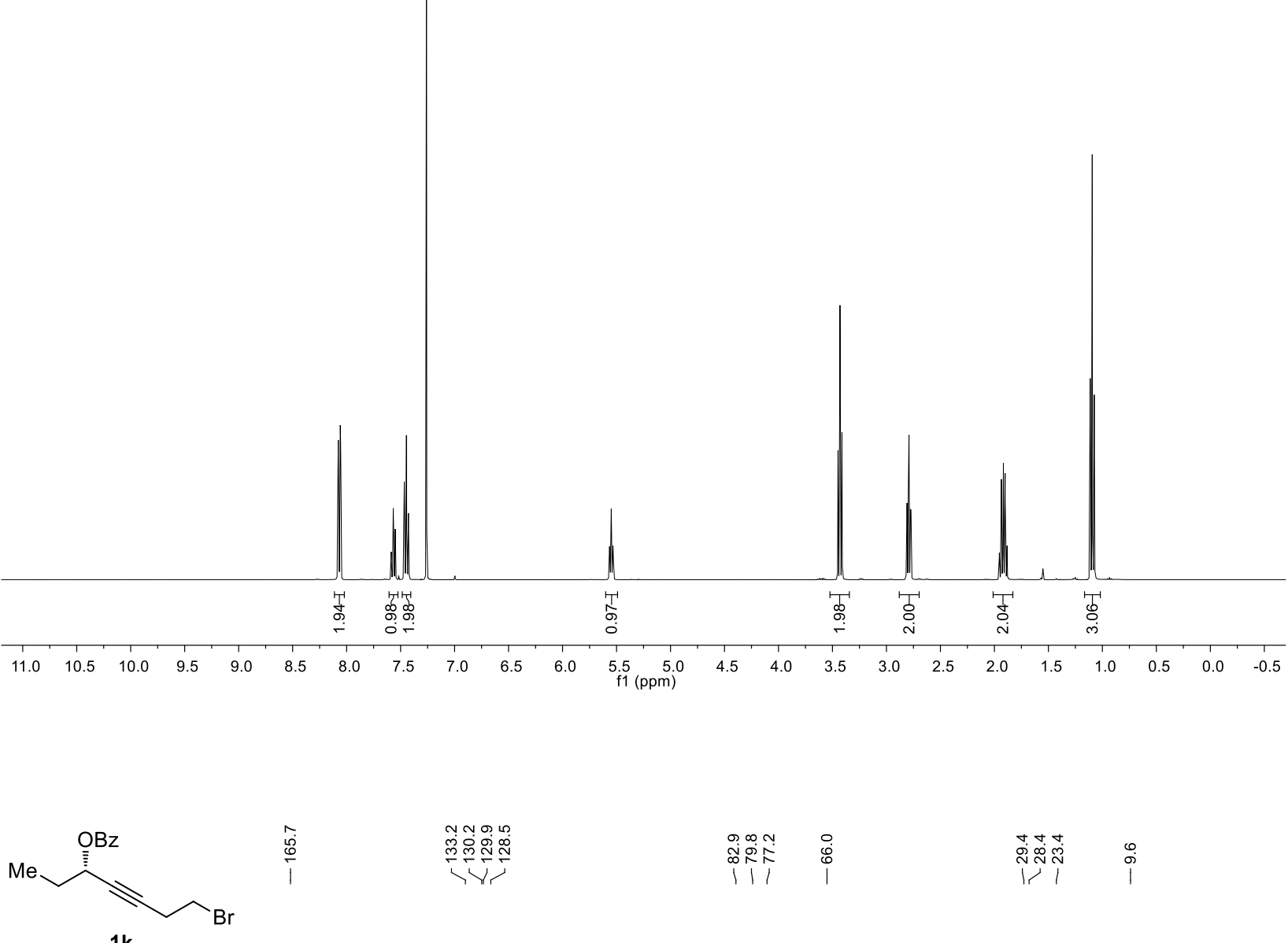

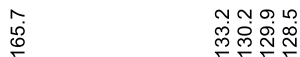

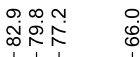

iั

${ }^{13} \mathrm{C}$ NMR, $101 \mathrm{MHz}$

$\mathrm{CDCl}_{3}, 298 \mathrm{~K}$

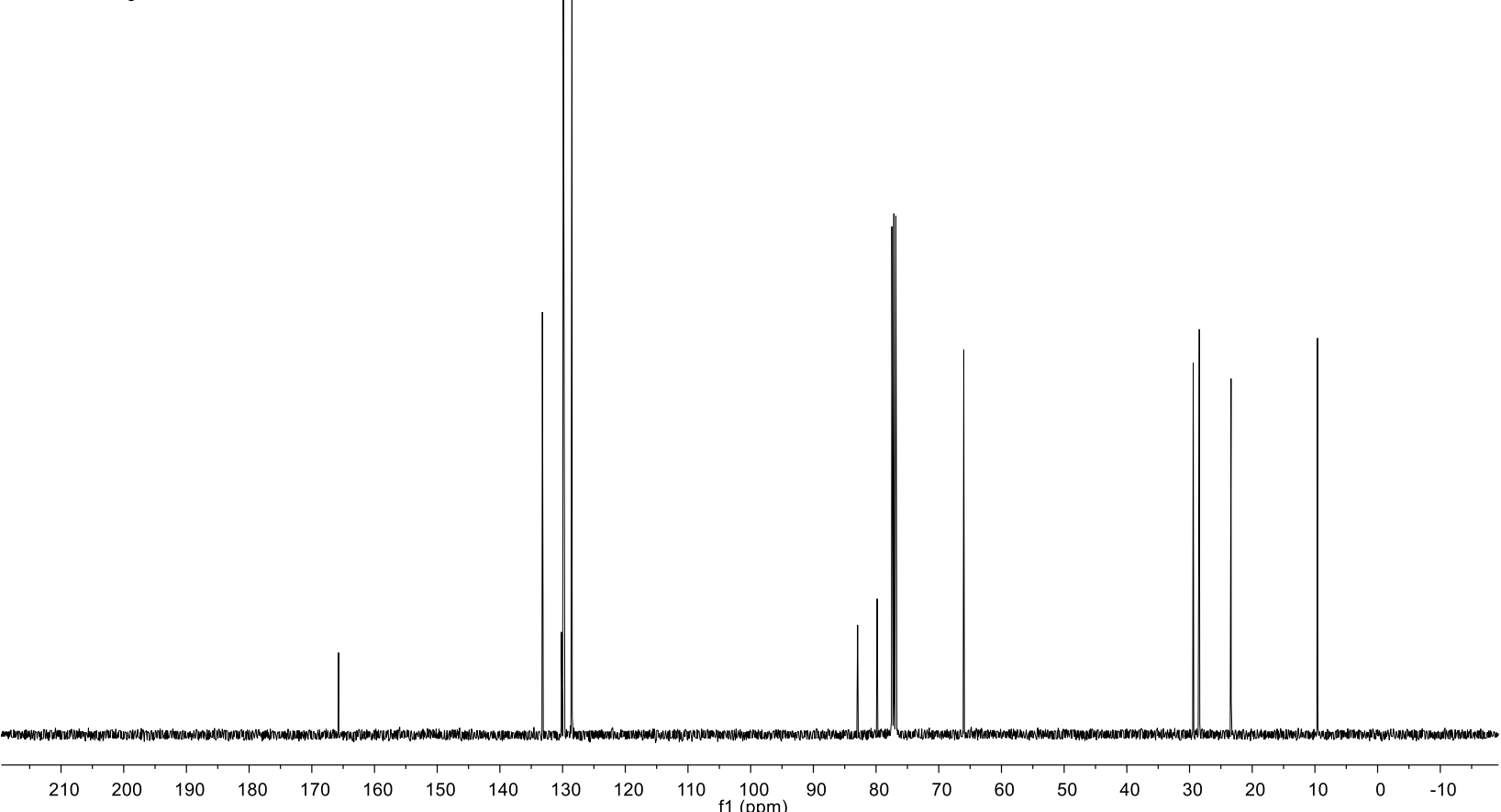


<smiles>CC(C)C=C=C(CCCOC(=O)OCc1ccccc1)c1ccccc1</smiles>

3a

${ }^{1} \mathrm{H} \mathrm{NMR}, 400 \mathrm{MHz}$

$\mathrm{CDCl}_{3}, 298 \mathrm{~K}$

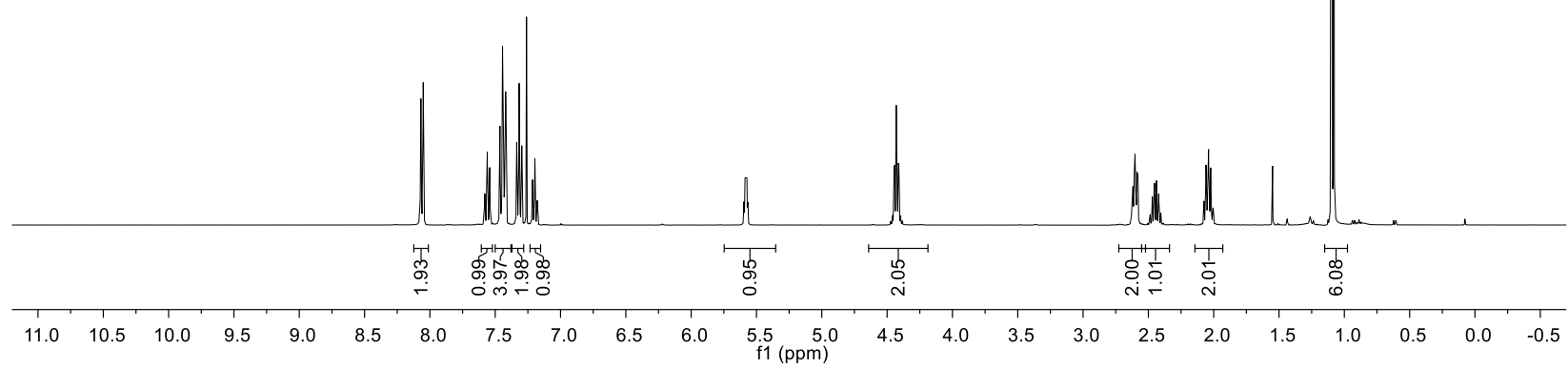

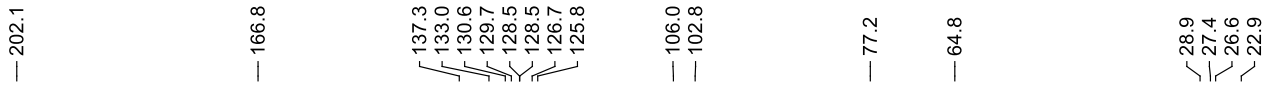<smiles>CC(C)C=C=C(CCCOC(=O)OCc1ccccc1)c1ccccc1</smiles>

3a

${ }^{13} \mathrm{C}$ NMR, $101 \mathrm{MHz}$

$\mathrm{CDCl}_{3}, 298 \mathrm{~K}$

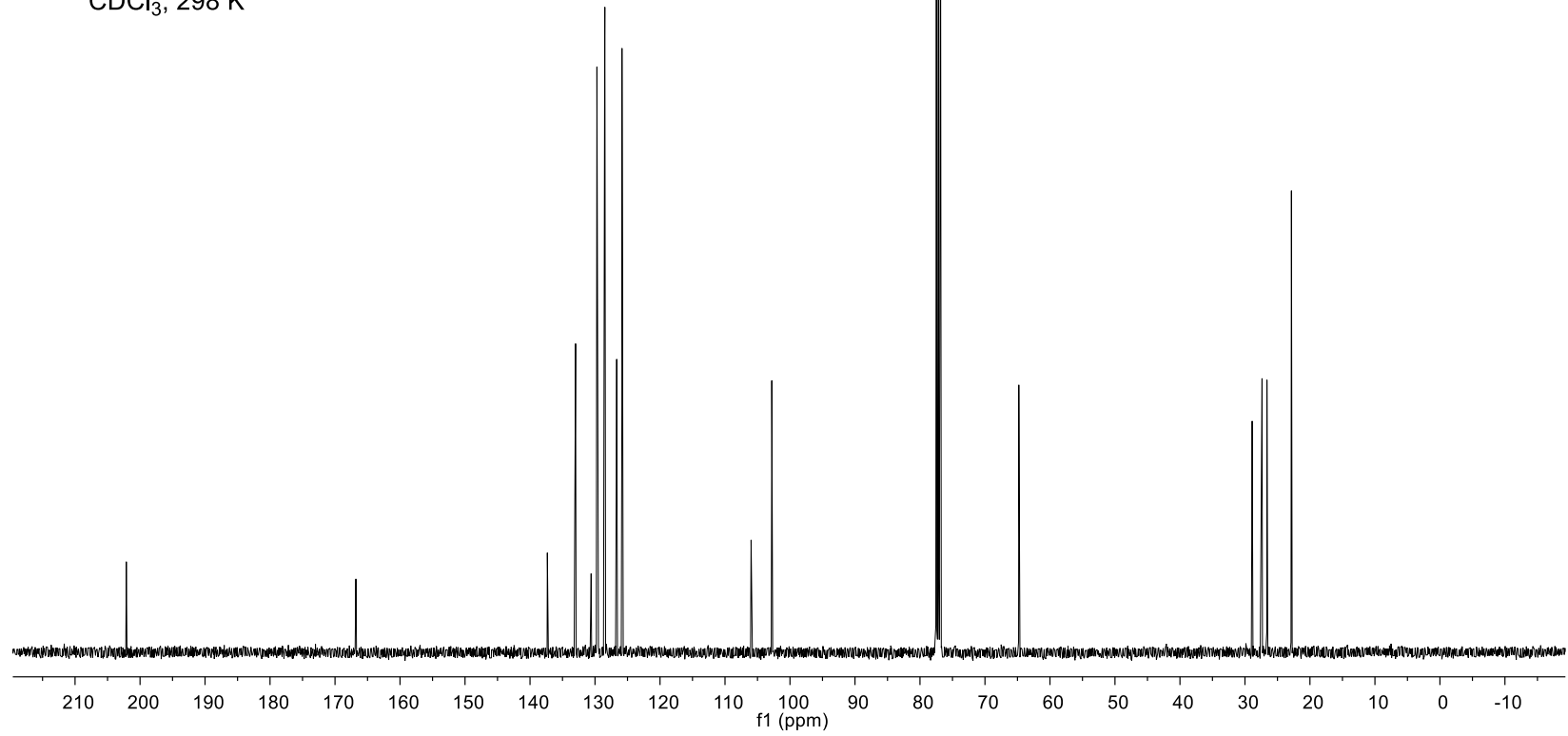




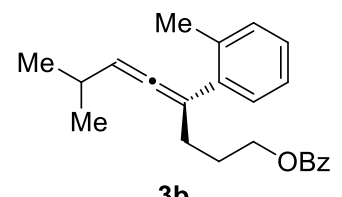

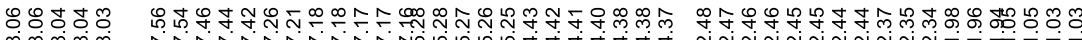

${ }^{1} \mathrm{H}$ NMR, $400 \mathrm{MHz}$

$\mathrm{CDCl}_{3}, 298 \mathrm{~K}$

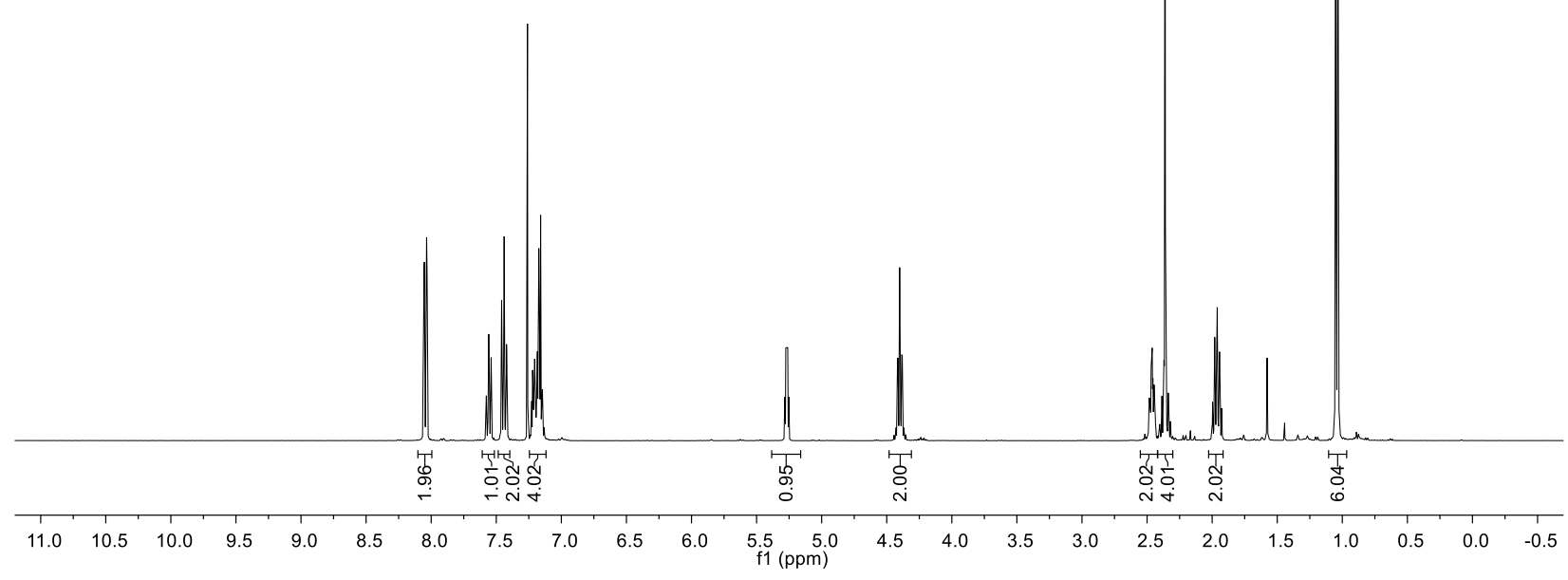

li<smiles>Cc1ccccc1C(=CC=CC(C)C)CCCOC(C)(C)C</smiles>

3b

${ }^{13} \mathrm{C} \mathrm{NMR}, 101 \mathrm{MHz}$

$\mathrm{CDCl}_{3}, 298 \mathrm{~K}$

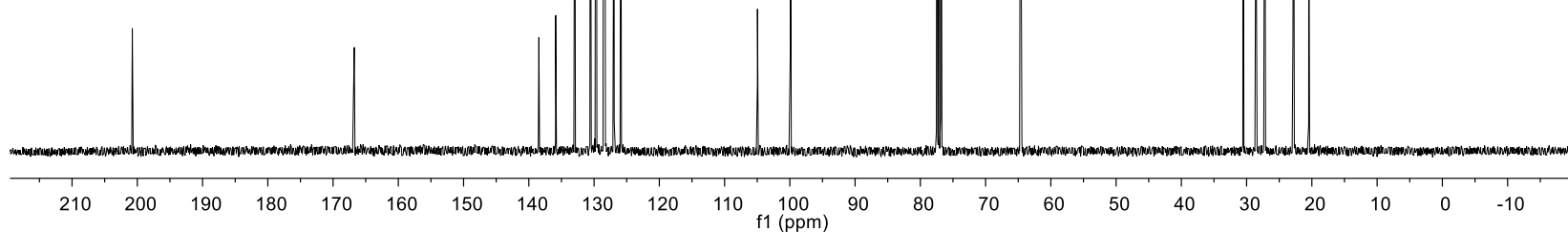




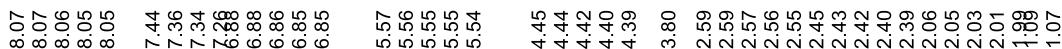

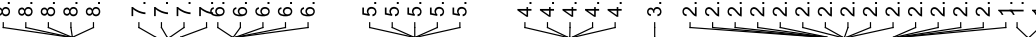

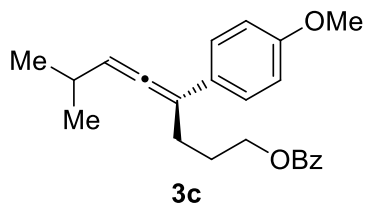

${ }^{1} \mathrm{H} \mathrm{NMR}, 400 \mathrm{MHz}$

$\mathrm{CDCl}_{3}, 298 \mathrm{~K}$

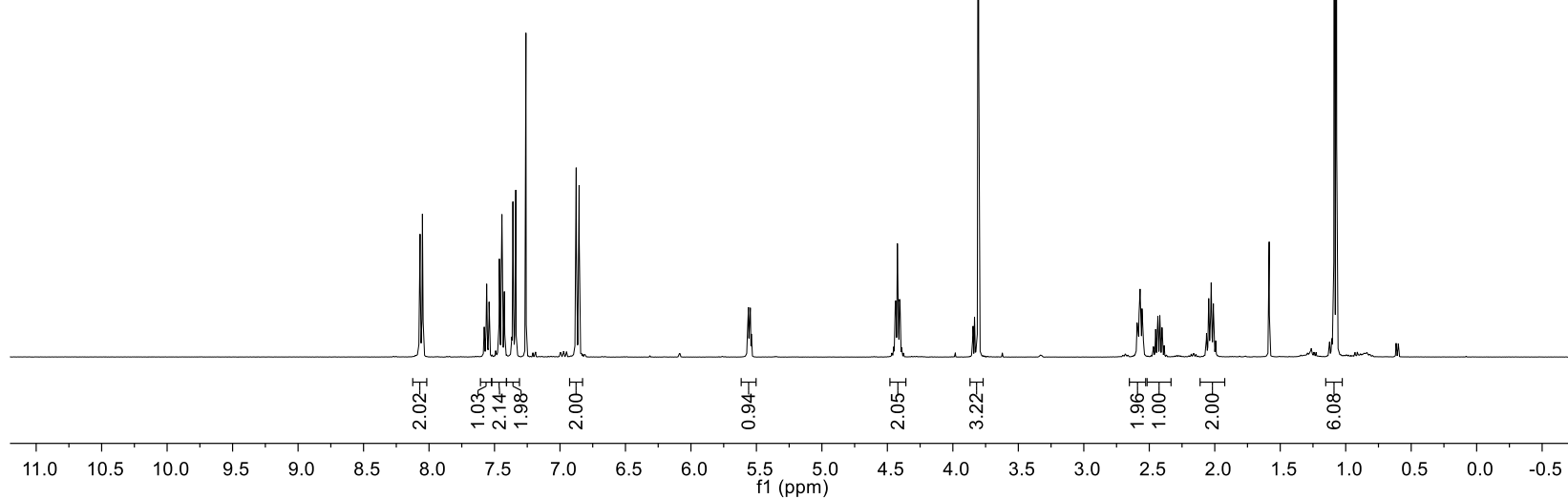

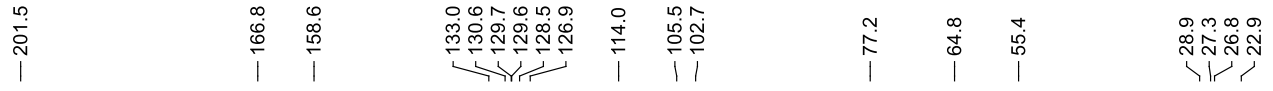<smiles>COc1ccc(C(=CCCOC(=O)OCc2ccccc2)CCC(C)C)cc1</smiles>

${ }^{13} \mathrm{C}$ NMR, $101 \mathrm{MHz}$

$\mathrm{CDCl}_{3}, 298 \mathrm{~K}$

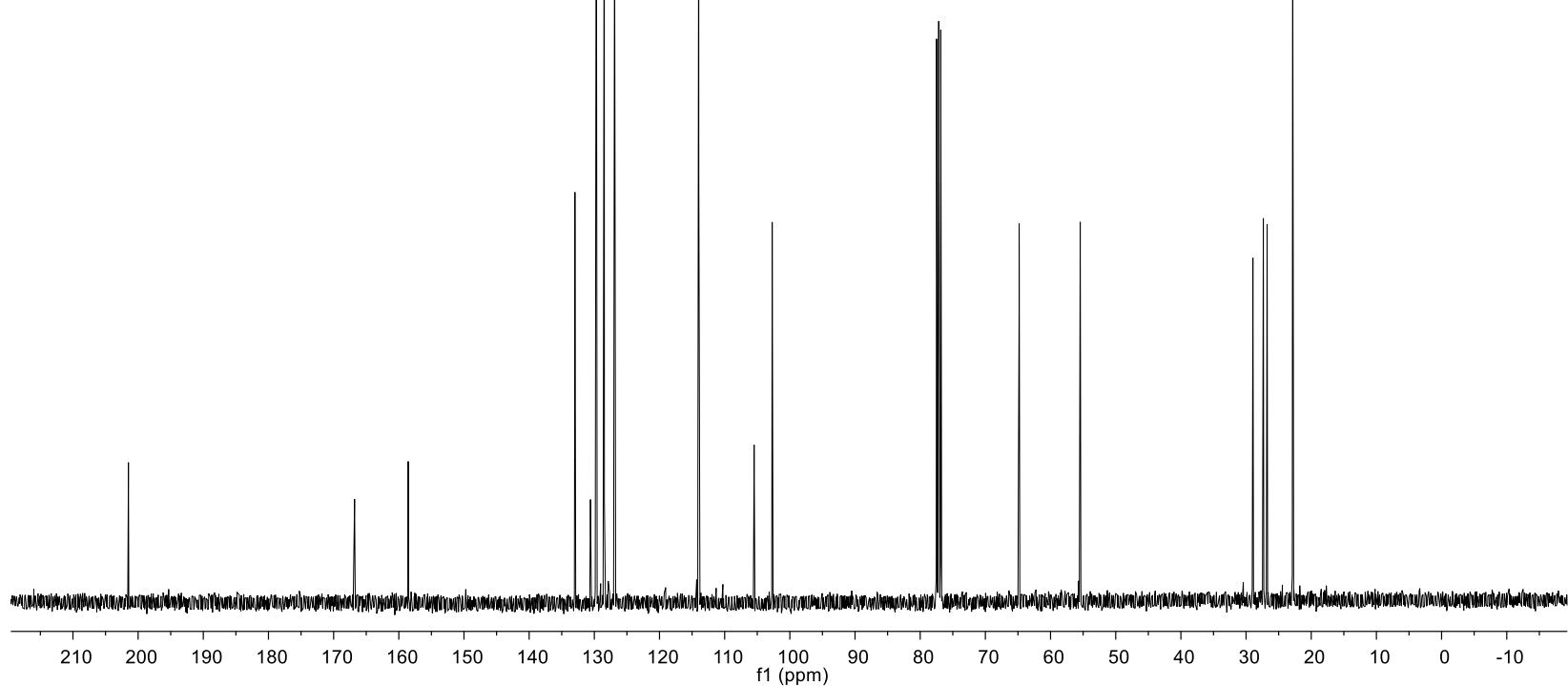


<smiles>CC(C)C=C=C(CCCOC(=O)OCc1ccccc1)c1ccc2c(c1)OCCO2</smiles>

${ }^{1} \mathrm{H}$ NMR, $400 \mathrm{MHz}$

$\mathrm{CDCl}_{3}, 298 \mathrm{~K}$

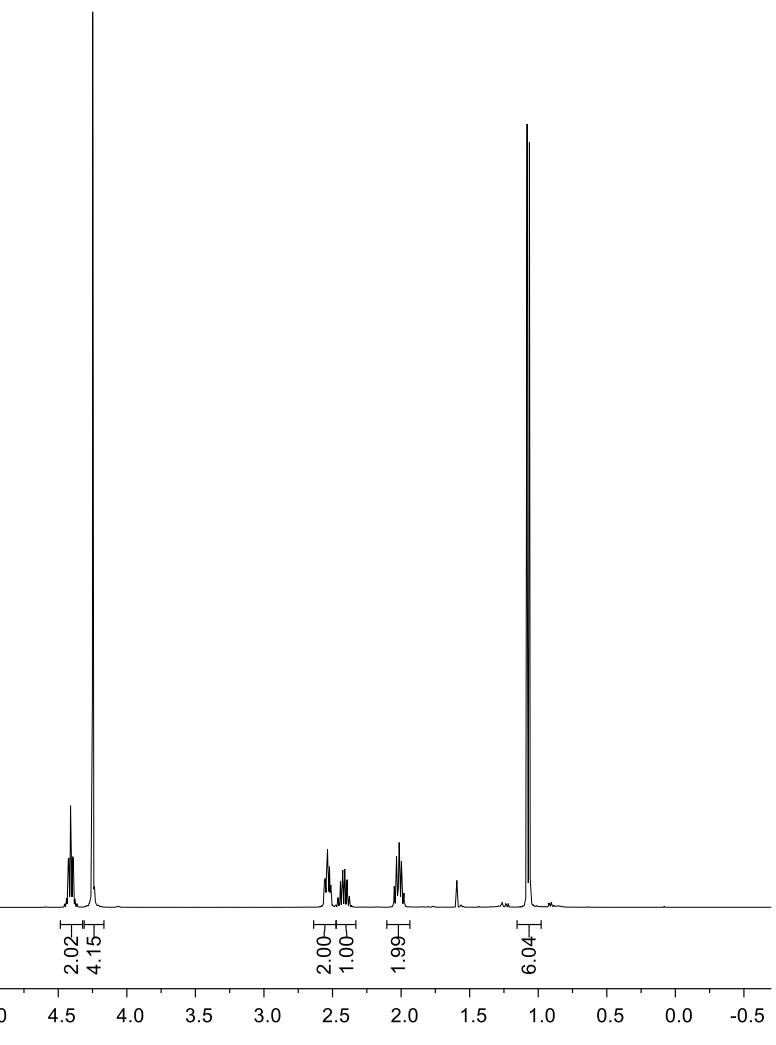

$\stackrel{\circ}{\grave{i}}$

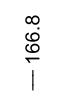

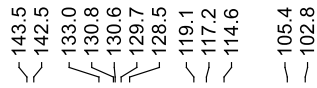

ĩ.

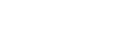<smiles>CC(C)C=C=C(CCCOC(C)(C)C)c1ccc2c(c1)OCCO2</smiles>

${ }^{13} \mathrm{C} \mathrm{NMR}, 101 \mathrm{MHz}$

$\mathrm{CDCl}_{3}, 298 \mathrm{~K}$

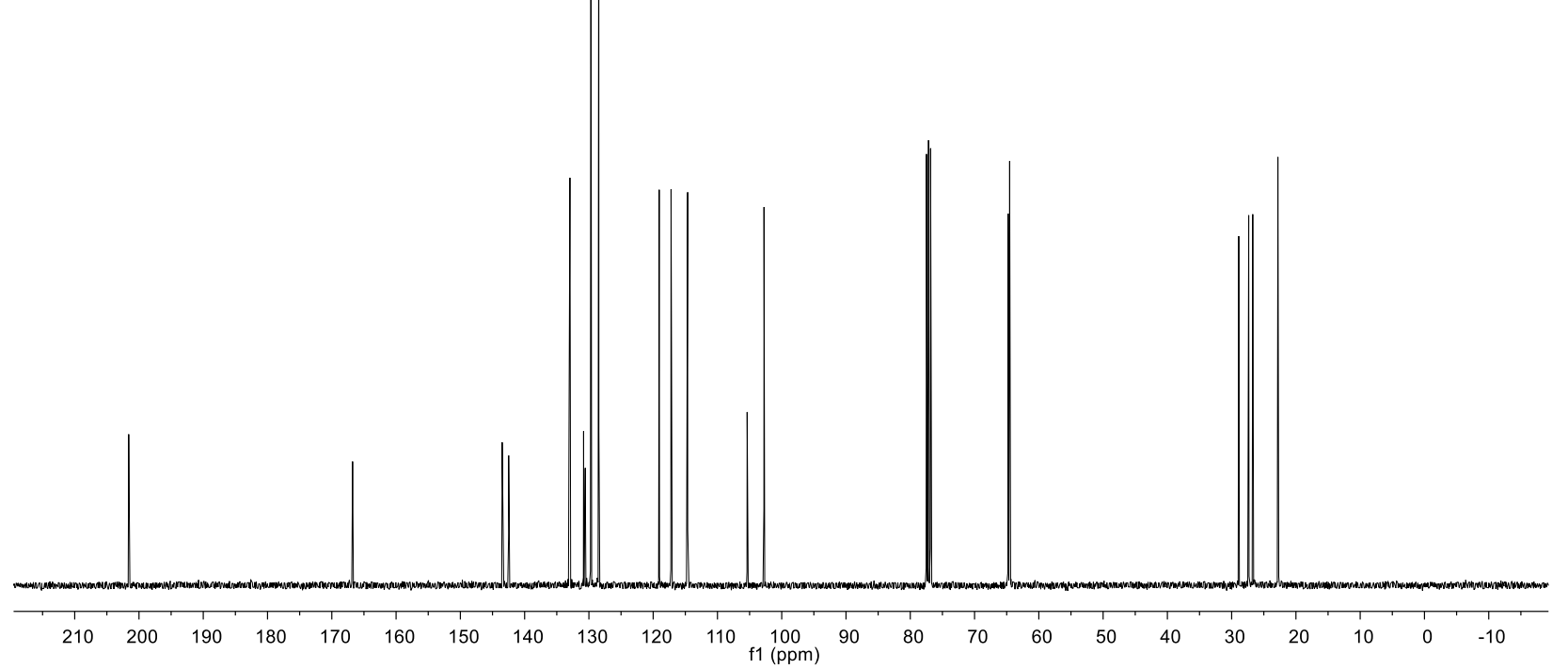


<smiles>CC(C)C=CC(=CCCOC(=O)OCc1ccccc1)c1cccc2ccccc12</smiles>

$3 e$

${ }^{1} \mathrm{H}$ NMR, $400 \mathrm{MHz}$

$\mathrm{CDCl}_{3}, 298 \mathrm{~K}$

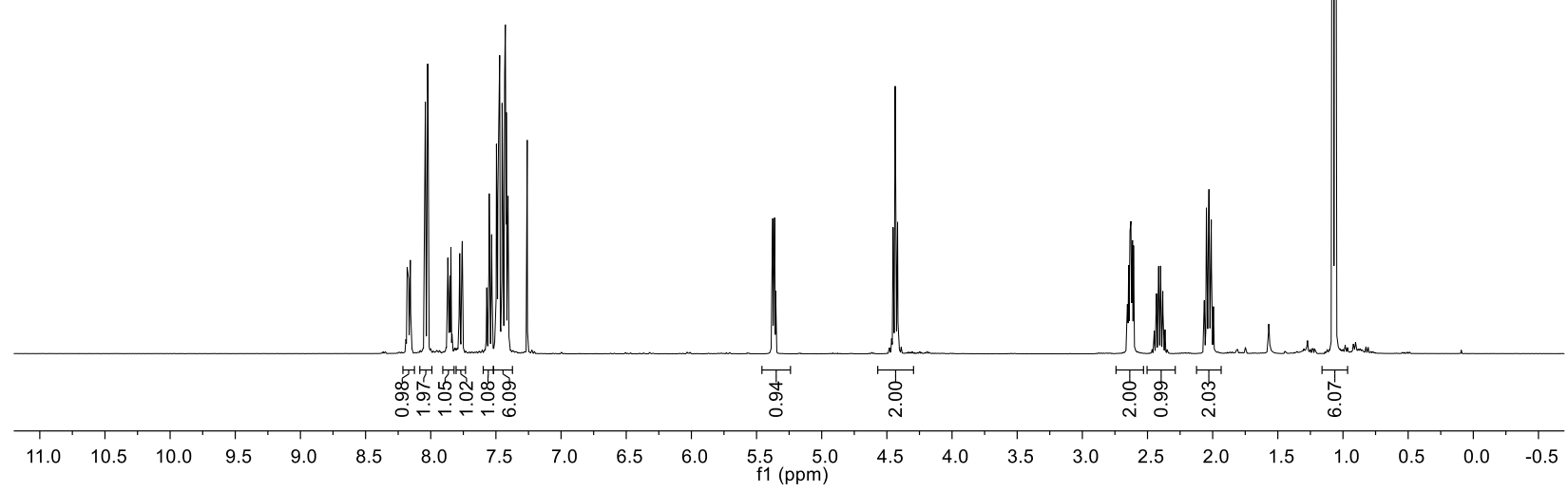

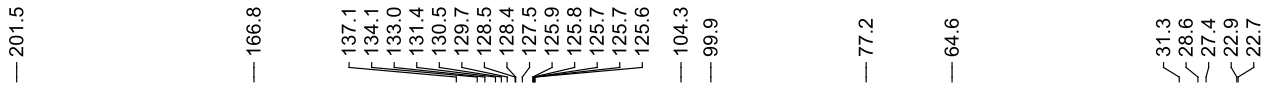<smiles>CC(C)C=C=C(CCCOC(=O)OCc1ccccc1)c1cccc2ccccc12</smiles>

$3 e$

${ }^{13} \mathrm{C} \mathrm{NMR}, 101 \mathrm{MHz}$

$\mathrm{CDCl}_{3}, 298 \mathrm{~K}$

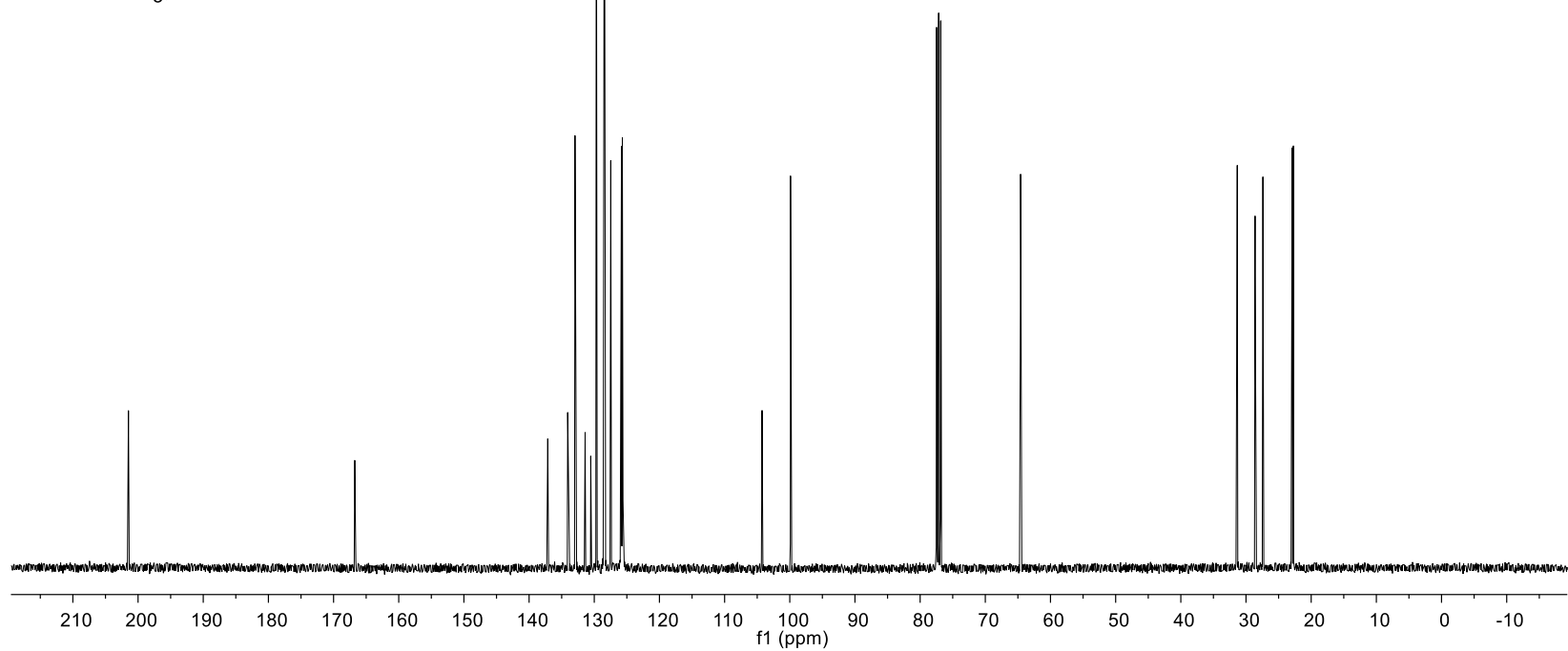


<smiles>CC(C)(C)C=CC(CN=[18O])c1ccc(Br)cc1</smiles>

${ }^{1} \mathrm{H} \mathrm{NMR}, 400 \mathrm{MHz}$

$\mathrm{CDCl}_{3}, 298 \mathrm{~K}$

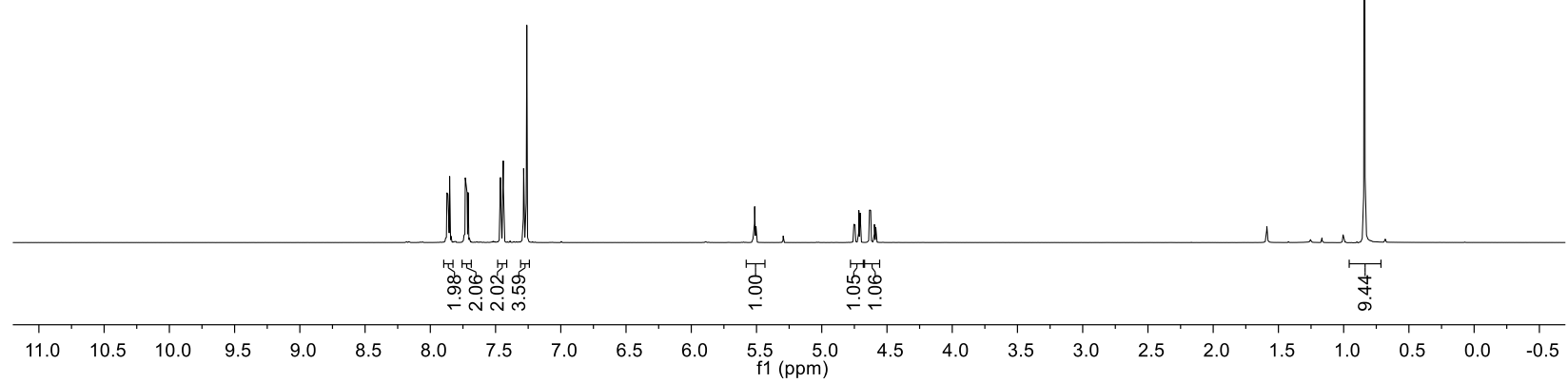

용

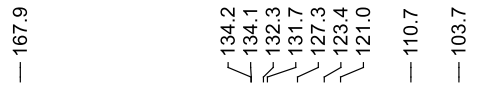

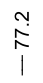

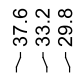<smiles>CC(C)(C)C=C=C(C[18OH])c1ccc(Br)cc1</smiles>

${ }^{13} \mathrm{C}$ NMR, $101 \mathrm{MHz}$

$\mathrm{CDCl}_{3}, 298 \mathrm{~K}$

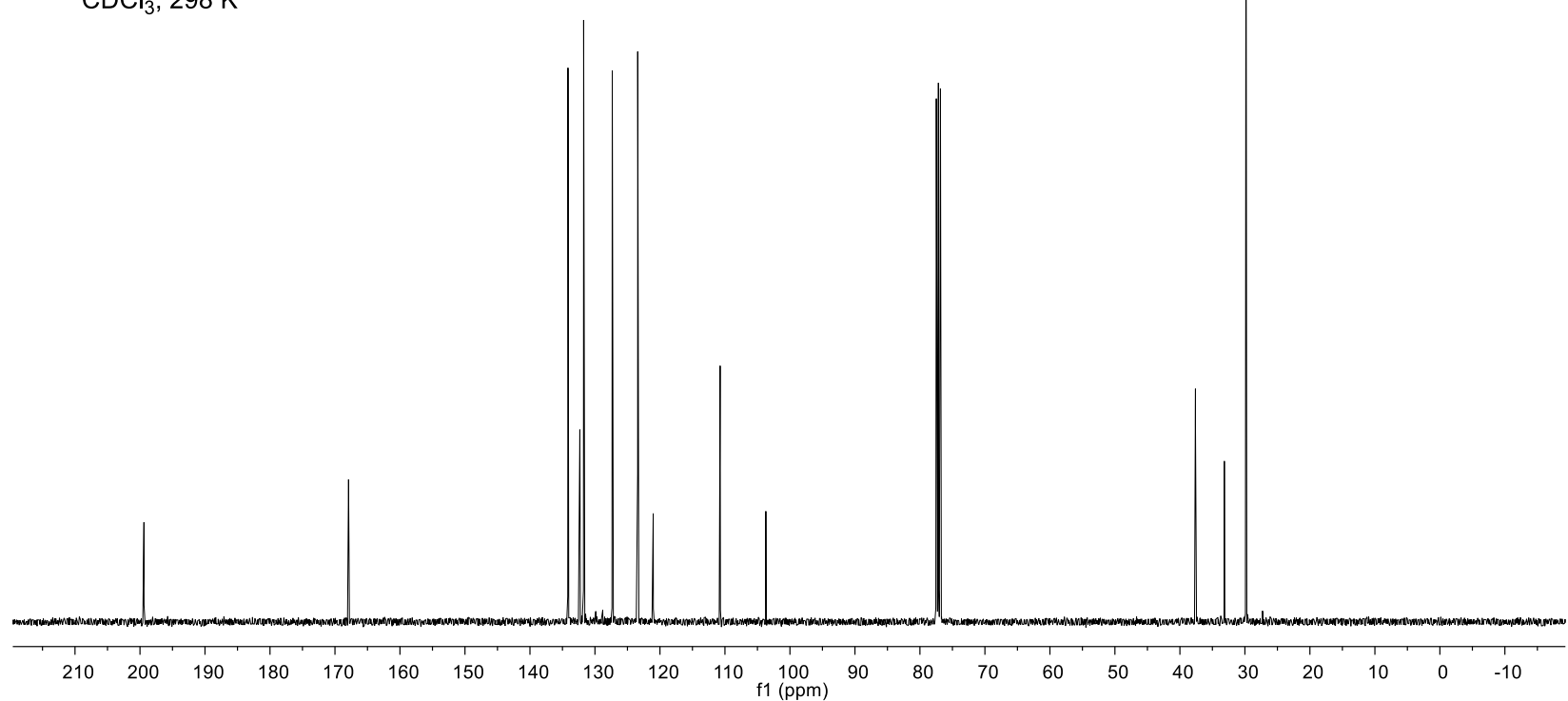


<smiles>CC(C)(C)C=C=C(CNc1ccccc1)c1ccc(-c2ccccc2)cc1</smiles>

3g

${ }^{1} \mathrm{H}$ NMR, $400 \mathrm{MHz}$

$\mathrm{CDCl}_{3}, 298 \mathrm{~K}$

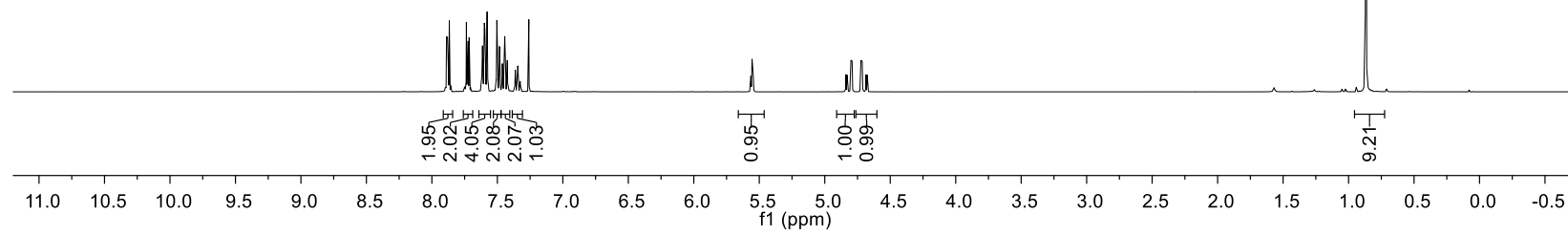

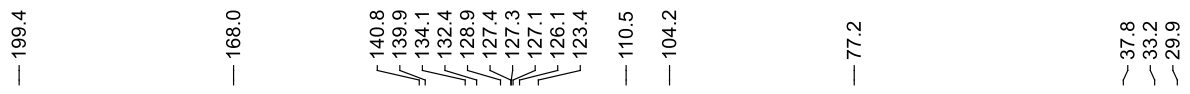<smiles>CC(C)(C)C=C=C(CNc1ccccc1)c1ccc(-c2ccccc2)cc1</smiles>

$3 \mathbf{g}$

${ }^{13} \mathrm{C}$ NMR, $101 \mathrm{MHz}$

$\mathrm{CDCl}_{3}, 298 \mathrm{~K}$

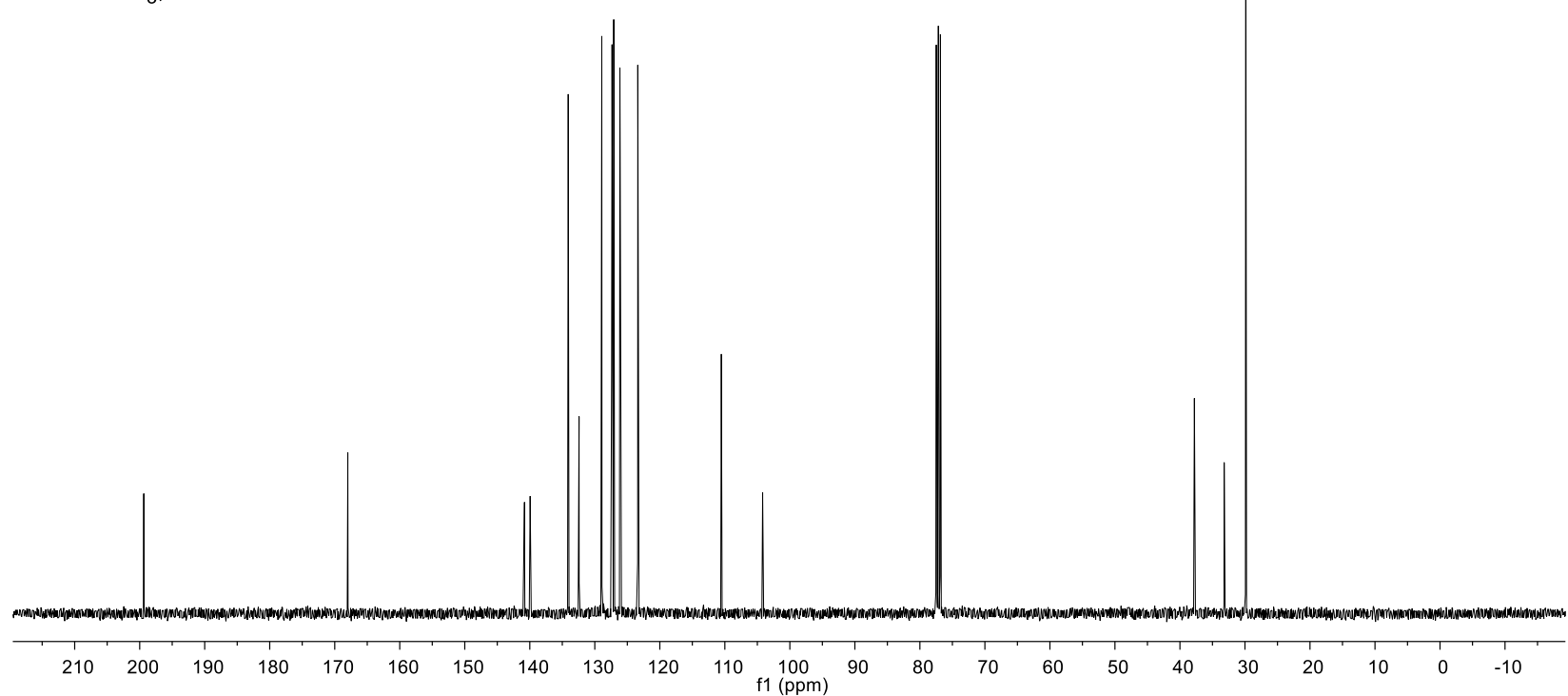


<smiles>COc1ccc(C(=CC(C)(C)C)C[NH+])cc1OC</smiles>

$3 \mathrm{~h}$

${ }^{1} \mathrm{H}$ NMR, $400 \mathrm{MHz}$

$\mathrm{CDCl}_{3}, 298 \mathrm{~K}$

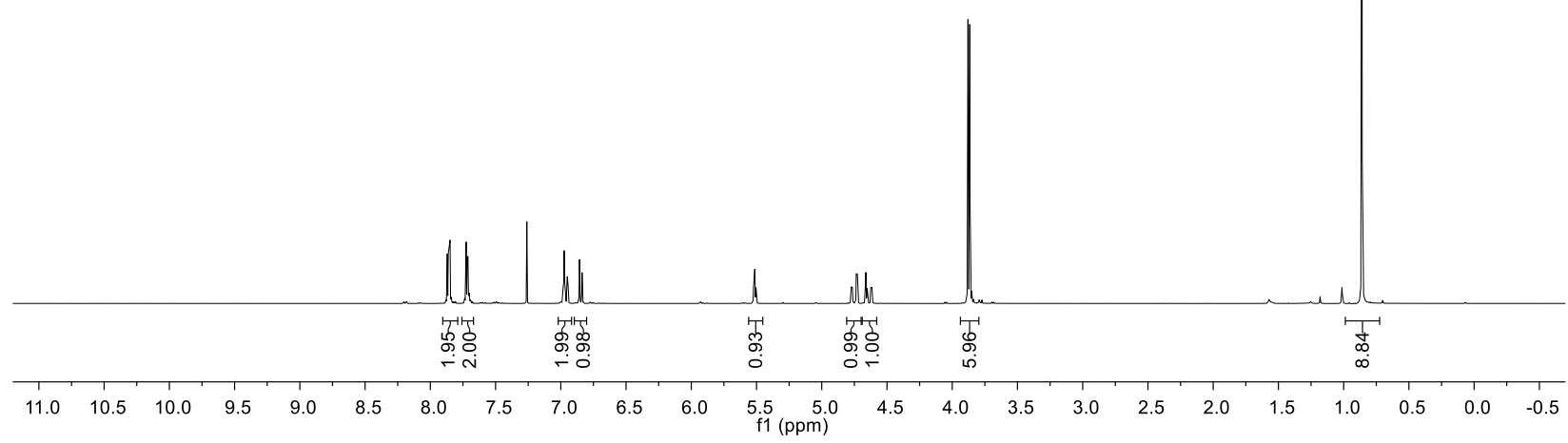

\begin{tabular}{|c|c|c|c|}
\hline & 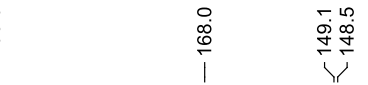 & 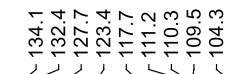 \\
\hline
\end{tabular}<smiles>COc1ccc(C(=CC(C)(C)C)CN=[NH+])cc1OC</smiles>

$3 \mathrm{~h}$

${ }^{13} \mathrm{C}$ NMR, $101 \mathrm{MHz}$

$\mathrm{CDCl}_{3}, 298 \mathrm{~K}$

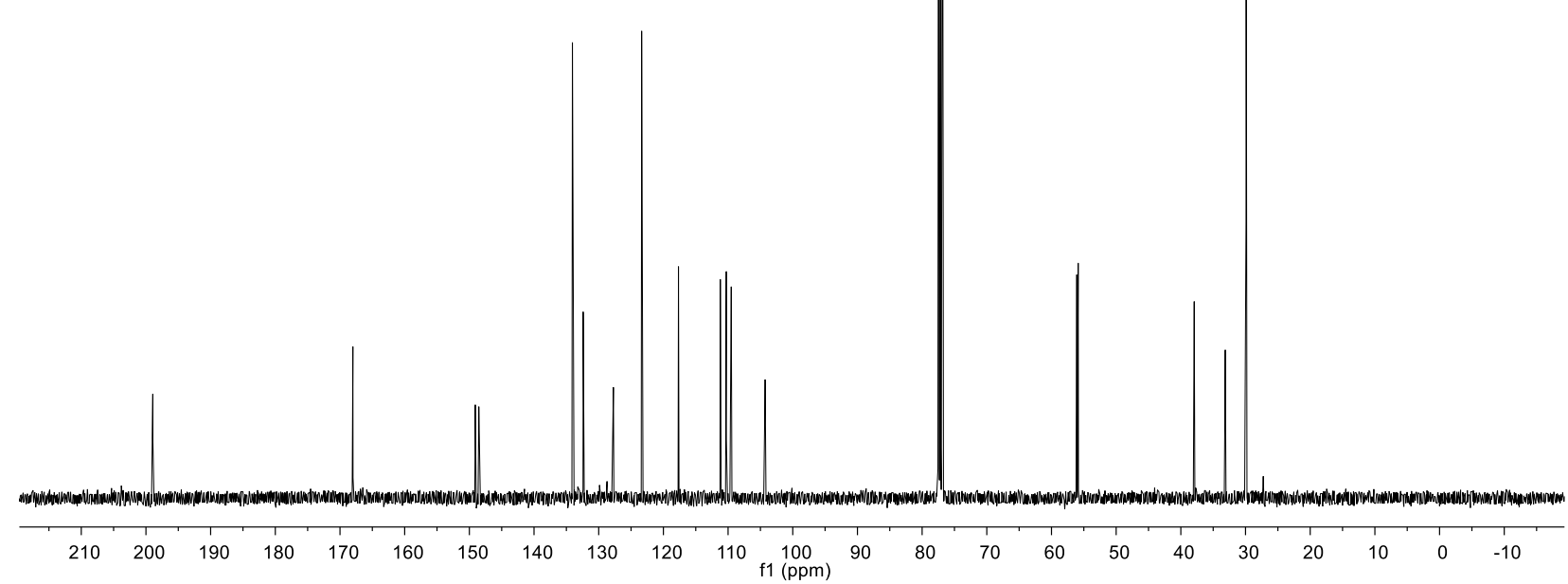




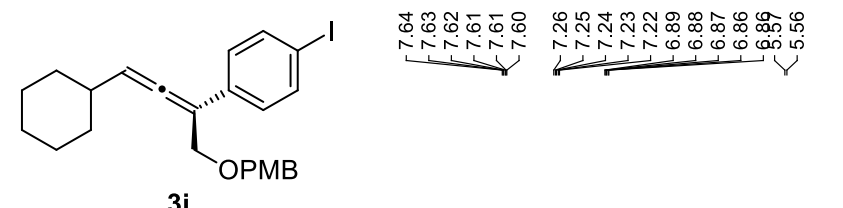

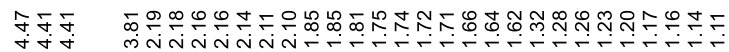

${ }^{1} \mathrm{H}$ NMR, $400 \mathrm{MHz}$

$\mathrm{CDCl}_{3}, 298 \mathrm{~K}$

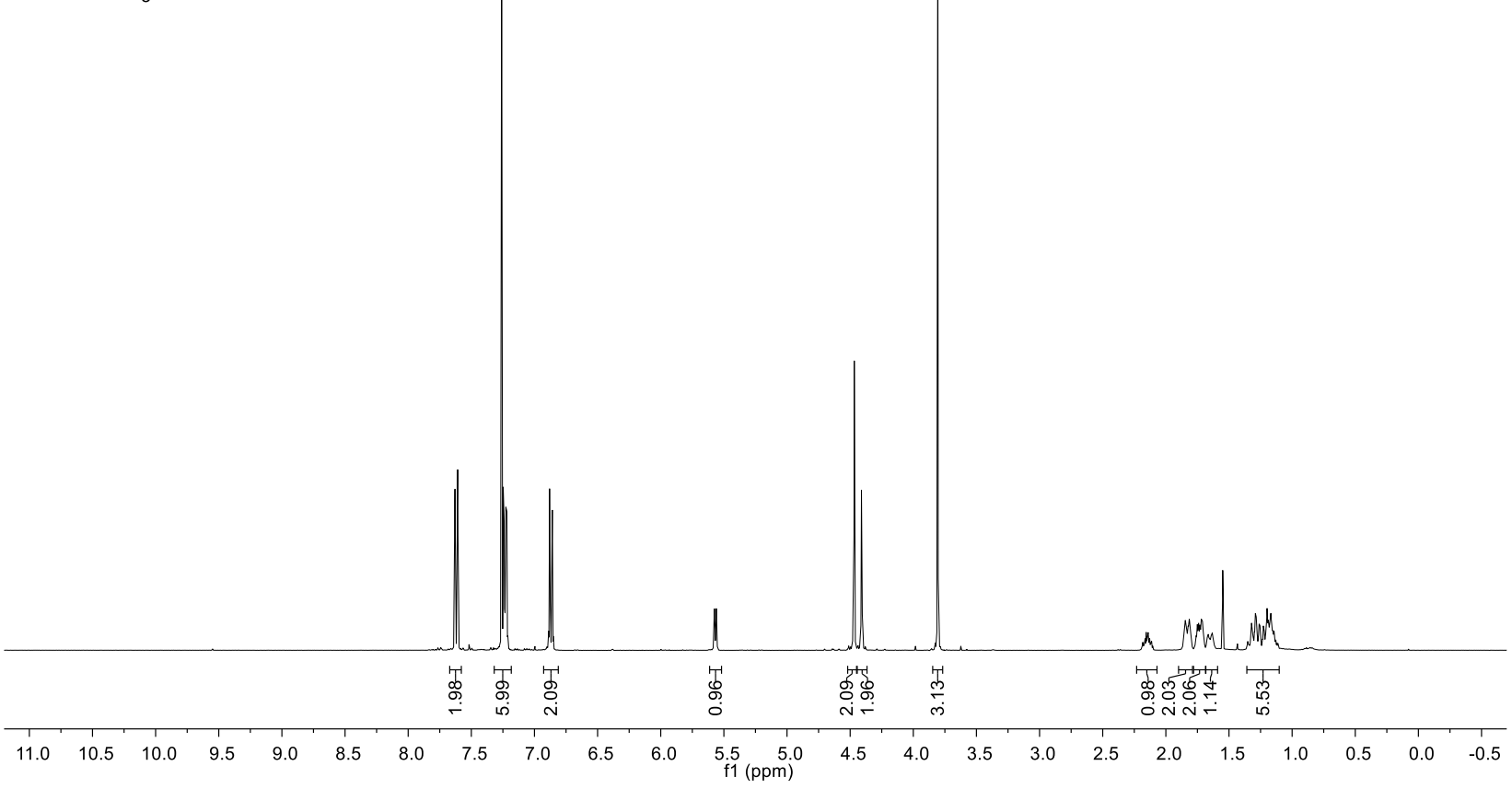

i

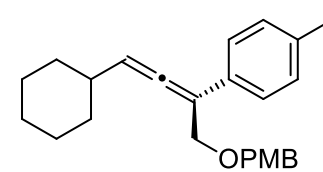

3i

${ }^{13} \mathrm{C} \mathrm{NMR}, 101 \mathrm{MHz}$

$\mathrm{CDCl}_{3}, 298 \mathrm{~K}$

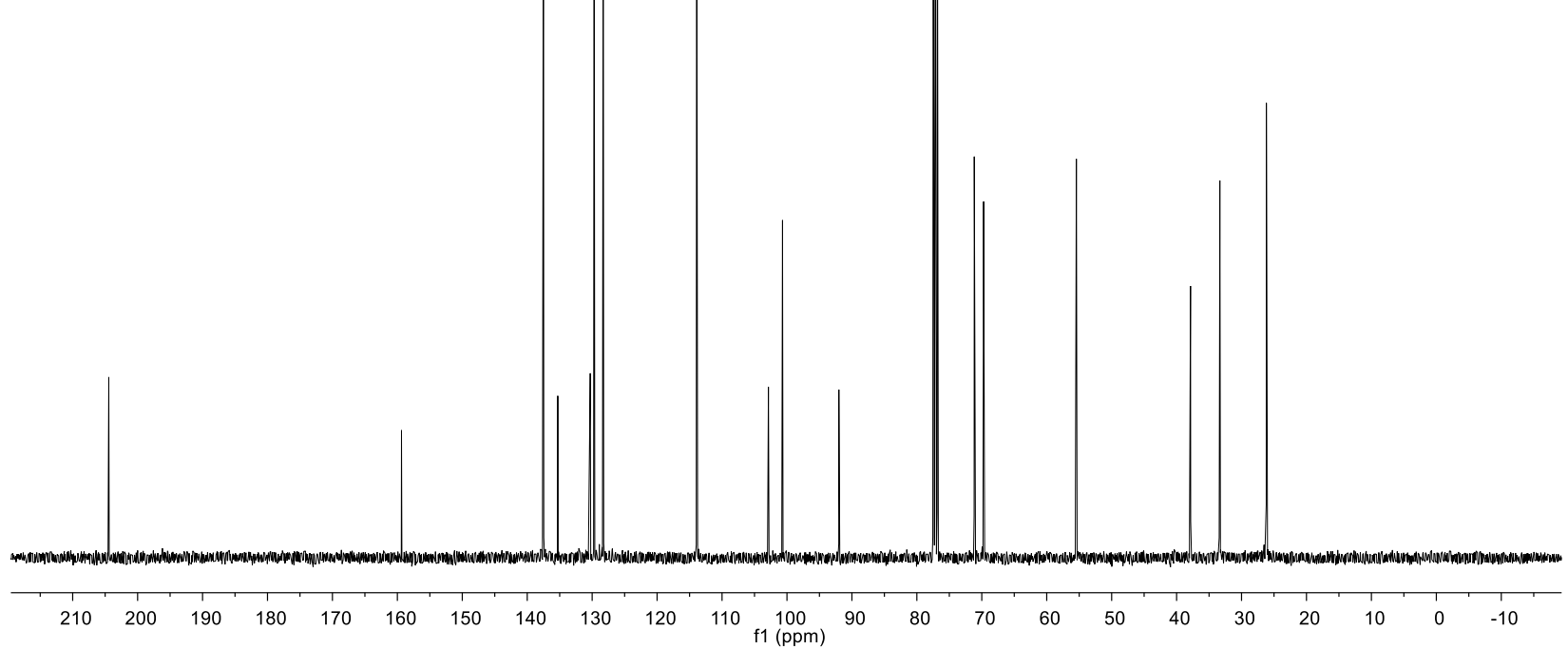


<smiles>[R16]OCC(=CC1CCCCC1)c1cccc(Br)c1</smiles>

3j

${ }^{1} \mathrm{H}$ NMR, $400 \mathrm{MHz}$

$\mathrm{CDCl}_{3}, 298 \mathrm{~K}$

i̊ํํ

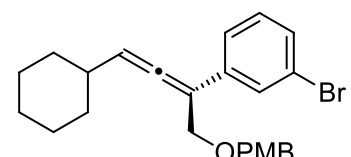

3j

${ }^{13} \mathrm{C}$ NMR, $101 \mathrm{MHz}$

$\mathrm{CDCl}_{3}, 298 \mathrm{~K}$

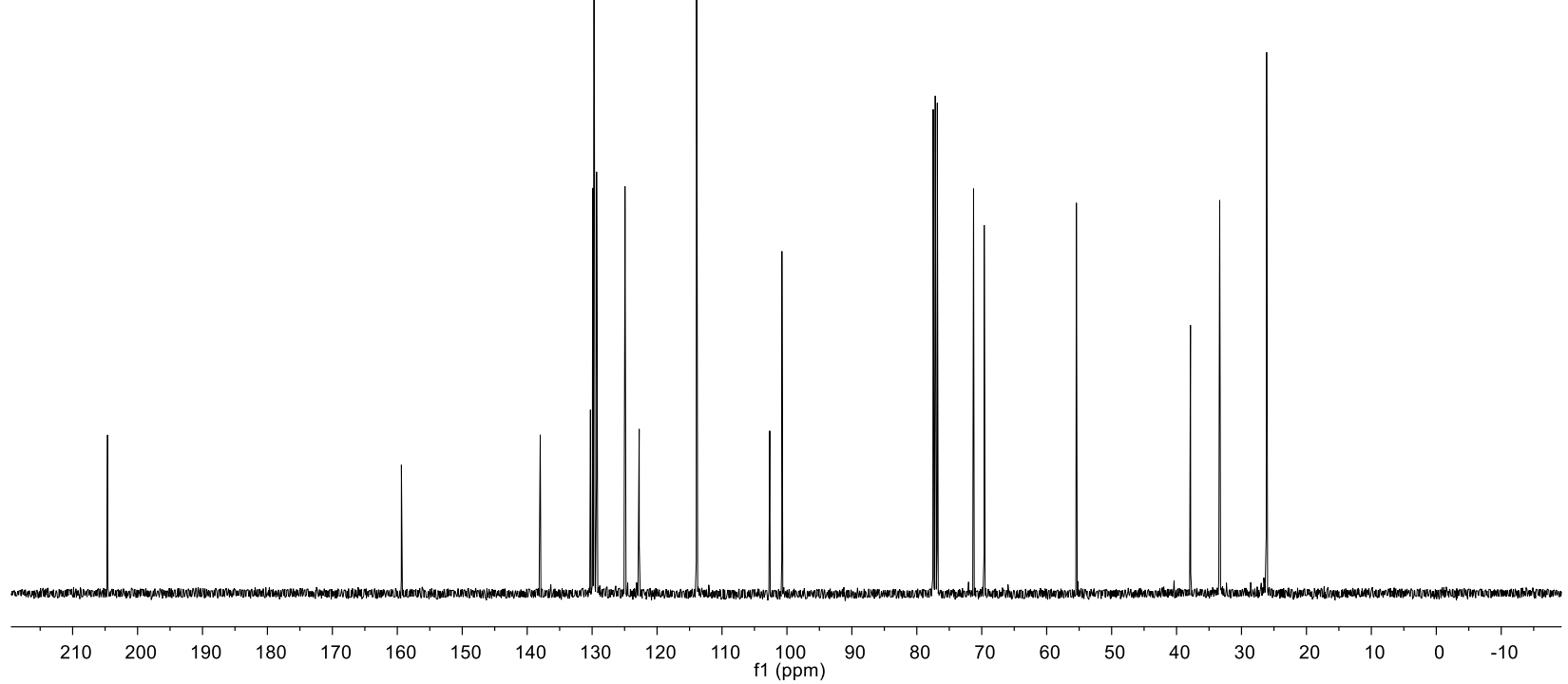




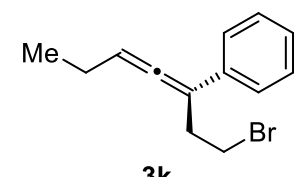

3k

${ }^{1} \mathrm{H}$ NMR, $400 \mathrm{MHz}$

$\mathrm{CDCl}_{3}, 298 \mathrm{~K}$

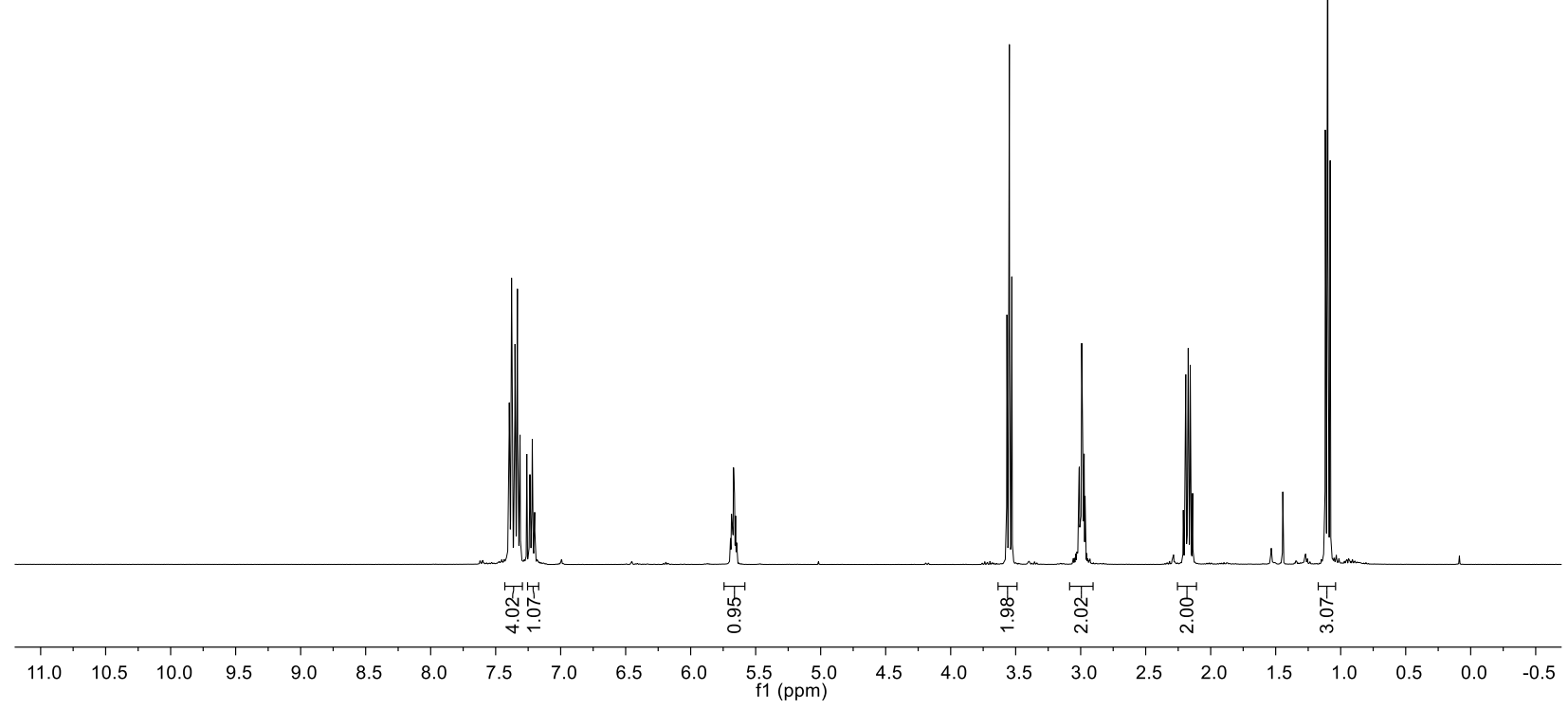

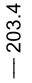

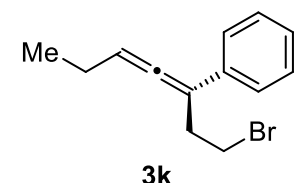

${ }^{13} \mathrm{C}$ NMR, $101 \mathrm{MHz}$

$\mathrm{CDCl}_{3}, 298 \mathrm{~K}$

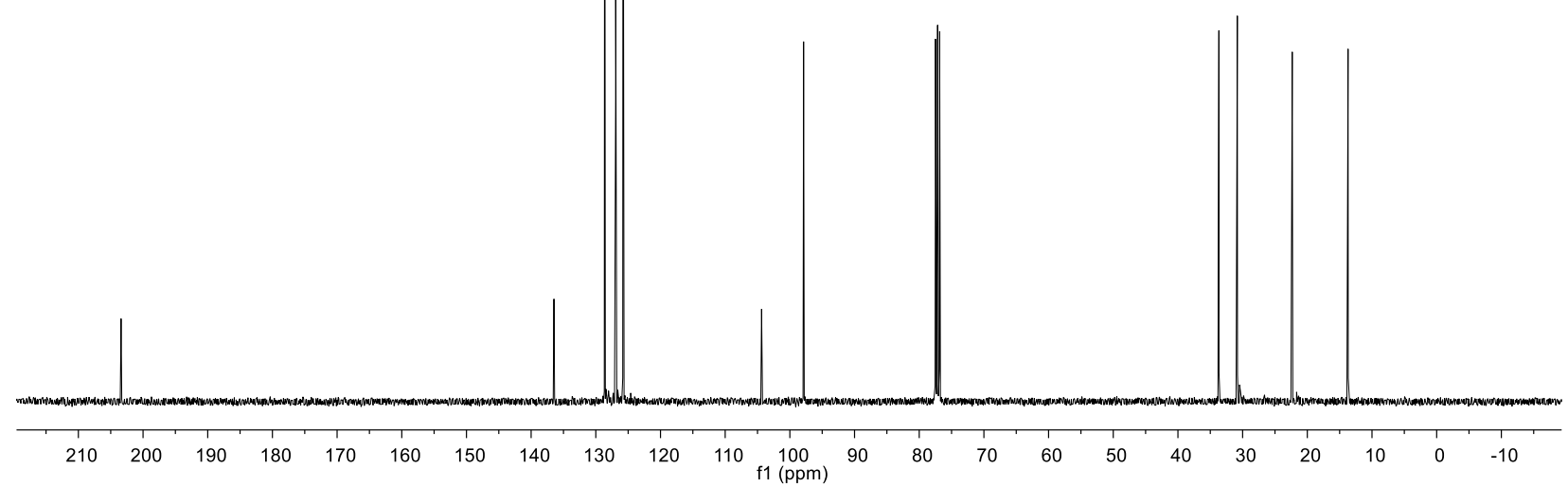

